\title{
SAMPLE SUPERVISED SEARCH CENTRIC APPROACHES IN GEOGRAPHIC OBJECT-BASED IMAGE ANALYSIS
}

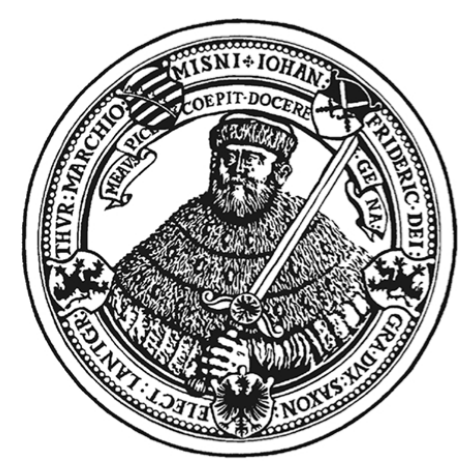

\section{Dissertation}

(kumulativ)

zur Erlangung des akademischen Grades doctor rerum naturalium

(Dr. rer. nat.)

vorgelegt dem Rat der Chemisch-Geowissenschaftlichen Fakultat der Friedrich-SchillerUniversitat Jena

von Christoffel Ettienne Fourie (M.Sc., Hons B.Sc., B.Sc.)

geboren am 18.03.1984 in Swellendam, Südafrika 
Gutachter:

1. PD Dr. Sören Hese

2. Prof. Dr. Raul Feitosa (Päpstliche Katholische Universität von Rio de Janeiro, Brasilien)

Tag der Verteidigung: 9. Dezember 2015 


\begin{abstract}
Sample supervised search centric image segmentation denotes a general method where quality segments are generated based on the provision of a selection of reference segments. The main purpose of such a method is to correctly segment a multitude of identical elements in an image based on these reference segments. An efficient search algorithm traverses the parameter space of a given segmentation algorithm. A supervised quality measure guides the search for the best segmentation results, or rather the best performing parameter set. This method, which is academically pursued in the context of remote sensing and elsewhere, shows promise in assisting the generation of earth observation information products. The method may find applications specifically within the context of user driven geographic objectbased image analysis approaches, mainly in respect of very high resolution optical data. Rapid mapping activities as well as general land-cover mapping or targeted element identification may benefit from such a method.

In this work it is suggested that sample supervised search centric geographic segment generation forms the basis of a set of methods, or rather a methodological avenue. The original formulation of the method, although promising, is limited in the quality of the segments it can produce - it is still limited by the inherent capability of the given segmentation algorithm. From an optimisation viewpoint, various structures may be encoded forming the fitness or search landscape traversed by a given search algorithm. Results are still measured with a given empirical discrepancy metric. These structures may interact or have an interplay with the given segmentation algorithm. Various method variants considering expanded fitness landscapes are possible. Additional processes, or constituents, such as data mapping, classification and post-segmentation heuristics may be embedded into such a method.
\end{abstract}

Here three different method variants are proposed. These novel methods aim to demonstrate the general feasibility of the proposed expanded fitness landscape design of sample supervised search centric geographic segment generation. The methods employ varied constituent types. All experiments throughout this work are conducted with three or four differentiated empirical discrepancy metrics that guide the search process. This is to prevent bias of any given metric towards any proposed method variant or sub-variant. Generally, results are presented demonstrating that the fitness/search landscapes generated by these expanded method designs are valid search problems. This is accomplished via parameter interdependency testing. Secondly, a selection of search methods is tested with all presented approaches, analysing if more complex search methods are needed to traverse the generated fitness landscapes. Specifically variants of differential evolution and particle swarm optimisation are used. Results highlight that the methods have variable interdependencies and that more complex metaheuristics are indeed needed as search methods (compared with, for example, random search and a hill climber).

The first presented method variant demonstrates the utility of encoding data mapping or transformation functions within the optimisation loop. Classical segmentation algorithms employed within remote sensing, such as multiresolution segmentation, start to have 
difficulties in segmenting multiple thematically similar elements when their spectral and/or geometric similarities start to diverge. A mapping function may tailor the data or the elements of interest in such a fashion that the given segmentation algorithm performs better on the given problem. Four data mapping functions are tested within such an approach. These mapping functions have varied characteristics. These functions consider variables, information through the data stack (multiband information) and also neighbourhood properties.

Results are presented with these four method variants, embedding data mapping, against the generic formulation of sample supervised segment generation. Four datasets were tested, consisting of informal and formal structure identification and cleared fields mapping tasks on very high resolution optical data. The computational impact of data mapping is negligible with most of the presented functions. Three of the four presented method variants, or rather mappings functions, showed promise in such a context. Specifically two mapping functions, namely the transformation matrix and genetic transform, showed extensive increases in segment quality in some problem instances (in excess of 0.20 in terms of reference weighted Jaccard scores).

As expected, no mapping function performed better under all conditions, as the selection of an appropriate function is still problem dependent. Nonetheless, both the transformation matrix and genetic transform functions provided statistically significantly better results in 24 of their 32 investigated problem instances. In the eight instances where results were not statistically significantly better, results were comparable or not statistically significantly worse than the generic method variant (not worse by 0.02 reference weighted Jaccard metric scores). This suggests that these method variants may be employed safely in a range of problems or under a range of metric conditions. It should be noted here that experimentation was conducted under generalizability conditions (two-fold cross validation and +250 million segment evaluations), giving a well-rounded/general indication of method usefulness.

The second method variant proposed considers graph-based connected component segmentation (mathematical morphology). Constrained connectivity, a variant of such segmentation based on the notion of a quasi-flat zone, defines spectral dissimilarity as a connectivity relation. The core segmentation algorithm consists of a two-dimensional parameter space constraining the growth of segments. This method may allow the encoding of additional attributes to threshold, or prevent the growth of segments. These attribute constraints are considered an additional constituent in an expanded fitness landscape design of sample supervised segment generation. Additionally, as with the presented method above, data mapping functions may also be encoded into such a method.

A method is thus proposed consisting of three constituents, namely the core constrained connectivity algorithm, a data mapping constituent and an attribute thresholding constituent. Six attributes are defined for consideration (increasing/monotonic), considering geometric and spectral characteristics that collectively form the attribute thresholding constituent. Here four differentiated method variants are presented, consisting of various constituent combinations $(\mathrm{CC}, \mathrm{CC}+\mathrm{Attr}, \mathrm{CC}+\mathrm{Map}$ and $\mathrm{CC}+\mathrm{Attr}+\mathrm{Map})$. These four method variants are contrasted 
among themselves to demonstrate their utility in similar extensive evaluation conditions as considered with the data mapping function method variant.

Results demonstrate that thresholdable attributes, initially conjectured to be safely useful, do not provide much benefit under cross-validated conditions. In contrast, constrained connectivity enhanced with a selection of data mapping functions provided excellent results. A statistical ranking placed constrained connectivity enhanced with data mapping as the most useful, followed by the most complex method variant, considering both data mapping and attributes. Additionally, the attribute constituent requires additional computation time. All three constituents display parameter or variable interdependencies. Although useful in some instances and even resulting in the best results in a select problem or two, thresholdable attributes need to be considered with caution in such a method.

The third proposed method variant considers the use of spectral data contained within provided reference segments. It is proposed to construct a method constituent based around using these data. A selection of method variants is proposed, having varied constituents using classification to tailor the data. Spectral data are utilised in a one-class classification process (non-parametric, non-linear) to generate a mask of potential elements of interest. This process forms part of the constituent or search landscape - it is thus modifiable or tunable. The mask is used to generate samples of a synthetic secondary class, which lead to a two-class classification generating a probability map. This process also forms part of the search landscape/constituent. In this implementation, five parameters define the nature of the probability map.

Four method variants are proposed and investigated using such a probability image to tailor the data for higher quality image segmentation. These variants consider different approaches to fuse or hybridise the probability image and original input image data. Some variants contain additional parameters to control the hybridisation. This leads to very modular methods, although the fitness landscape dimensionality may increase substantially. As with the previous presented methods, extensive evaluations are conducted. In this instance (with the removal of a problematic empirical discrepancy metric) all method variants performed better than the generic variant of sample supervised segment generation. One method in particular, called Hybrid:CP, considers very modular interactions between the probability image and original input image and displays very promising results. Unfortunately, these methods add a substantial computational impact compared with the generic method formulation. This is due to the inclusion of classification (support vector machines). The problem may be alleviated by considering classifiers with faster training times.

These presented methods consider diverse manners in which sample supervised search centric geographic image segmentation may be defined, considering additional constituents for an expanded fitness landscape. Generally, most proposed variants performed better than the original formulation under extensive evaluation conditions. These results suggest such method designs, based on expanded fitness landscapes, or rather these sample supervised, search centric approaches in geographic object-based image analysis, are promising and worthy of further investigation. 


\section{ZUSAMMENFASSUNG}

Die stichprobenüberwachte suchbasierte Bildsegmentierung ist eine allgemeine Methode, bei der (so genannte) Qualitätssegmente auf der Grundlage einer Auswahl von Referenzsegmenten generiert werden. Die prinzipielle Aufgabe eines solchen Ansatzes ist die korrekte Segmentierung einer Vielzahl identischer Elemente in einem Bild auf der Grundlage dieser Referenzsegmente. Ein effizienter Suchalgorithmus traversiert den Parameterraum des gegebenen Segmentierungsalgorithmus, wobei ein überwachtes Qualitätsmaß die Suche nach den besten Segmentierungsergebnissen oder vielmehr dem performantesten Parametersatz lenkt. Diese Methode wird im Kontext der Fernerkundung und weiteren Bereichen erforscht und zeigt sich als vielversprechend bei der Unterstützung der Erstellung von auf Erdbeobachtung basierenden Informationsprodukten. Die Methode könnte speziell im Bereich der überwachten geographischen objektbasierten Bildanalyse, hauptsächlich im Kontext von sehr hochauflösenden optischen Satellitendaten, Anwendung finden. Sowohl Notfall- und Krisenkartierungen als auch die generelle Klassifikation von Landbedeckung oder die Detektion von Zielelementen können von dieser Methodik profitieren.

In der vorliegenden Arbeit wird die stichprobenüberwachte suchbasierte geographische Segmentgenerierung als eine Basis oder vielmehr als ein generelles methodisches Konzept für einer Menge von Methoden vorgeschlagen. Wenngleich die ursprüngliche Formulierung der Methode vielversprechend erscheint, ist sie, aufgrund ihrer Abhängigkeit von den inhärenten Eigenschaften des gegebenen Segmentierungsalgorithmus, hinsichtlich der Qualität der generierten Segmente limitiert. Im Kontext von Optimierung können diverse Strukturen encodiert werden, die die Fitness oder die zu traversierende Suchlandschaft eines bestimmten Suchalgorithmus formen. Die Ergebnisse werden weiterhin mittels eines vorgegebenen empirischen Diskrepanzmaßes evaluiert. Diese Strukturen können sowohl untereinander oder auch mit dem Segmentierungsalgorithmus selbst interagieren. Verschiedenste Varianten der Methode unter Verwendung von erweiterten Fitnesslandschaften sind möglich. Zusätzliche Prozesse (Komponenten), wie beispielsweise Datenabbildung (Mapping), Klassifikation sowie Heuristiken für eine Post-Segmentierung können in eine solche Methode integriert werden.

Hier werden drei verschiedene Methodenvarianten vorgeschlagen. Mittels dieser neuen Methoden soll die generelle Realisierbarkeit des vorgeschlagenen erweiterten Designs von Fitnesslandschaften für die stichprobenüberwachte suchbasierte geographische Segmentgenerierung demonstriert werden. Die Methoden verwenden vielfältige Komponententypen. Alle Experimente in dieser Arbeit werden mit drei beziehungsweise vier unterschiedlichen, den Suchprozess lenkenden, empirischen Diskrepanzmetriken durchgeführt, um eine Beeinflussung der gegebenen Metriken hinsichtlich aller vorgeschlagenen Methodenvarianten und Untervarianten $\mathrm{zu}$ vermeiden. Hauptsächlich belegen die dargelegten Ergebnisse, dass die von den erweiterten Methoden generierten Fitness- beziehungsweise Suchlandschaften valide Suchprobleme repräsentieren. Dieser Nachweis erfolgt durch den Test der Parameter auf gegenseitige Abhängigkeit. Des Weiteren wird eine Auswahl von Suchmethoden mit allen präsentierten Ansätzen getestet und 
analysiert, ob noch komplexere Ansätze erforderlich sind, um die jeweils generierten Fitnesslandschaften zielführend zu traversieren. Im Besonderen werden Optimierungsansätze basierend auf differenzieller Evolution sowie Partikelschwärmen verwendet. Die Ergebnisse verdeutlichen, dass die Parameter der Methoden Abhängigkeiten untereinander aufweisen und dass - beispielsweise verglichen mit einer zufälligen Suche oder dem Bergsteigeralgorithmus - in der Tat komplexere Metaheuristiken erforderlich sind.

Die Ergebnisse der ersten vorgestellten Methodenvariante belegen die Nützlichkeit der Encodierung von Datenabbildung oder Transformationsfunktionen innerhalb der Optimierung. Klassische Segmentierungsverfahren im Bereich Fernerkundung, wie beispielsweise die Multiskalen-Segmentierung, generieren schlechtere Ergebnisse bei der Segmentierung multipler thematisch ähnlicher Elemente sobald sie spektral und/oder geometrisch beginnen zu divergieren. Eine Abbildungsfunktion kann die zu verarbeitenden Daten oder Elemente jedoch derart anpassen, dass der gegebene Segmentierungsalgorithmus bessere Ergebnisse für die jeweilige Problemstellung liefert. Vier charakteristisch unterschiedliche Abbildungs-funktionen, die Variablen, Mehrkanal-Informationen und Nachbarschaftseigenschaften einbeziehen, werden in einem solchen Ansatz getestet.

Die Ergebnisse dieser vier Methodenvarianten zur Integration von Datenabbildung werden denen der generischen Formulierung der stichprobenüberwachten Segmentgenerierung gegenüber gestellt. Vier verschiedene, sehr hochauflösende optische Datensätze wurden mit dem Ziel der informellen und formellen Strukturidentifikation sowie der Feldkartierung getestet. Der rechnerische Mehraufwand der Datenabbildung hat sich für die meisten der präsentierten Funktionen als vernachlässigbar herausgestellt. Drei der vier präsentierten Methodenvarianten oder vielmehr Abbildungsfunktionen haben sich in den Experimenten als vielversprechend gezeigt. Insbesondere zwei Abbildungsfunktionen - die Transformationsmatrix und die genetische Transformation - haben für einige Problemstellungen einen beträchtlichen Anstieg der Segmentqualität bewirkt (über 0,20 bezüglich gewichteter JaccardKoeffizienten).

Wie erwartet, führte keine der Abbildungsfunktionen unter allen Bedingungen zu den besten Resultaten, da die Wahl einer geeigneten Funktion von der jeweiligen Problemstellung abhängt. Nichtsdestotrotz führten sowohl die Transformationsmatrix als auch die genetischen Transformationsfunktionen in 24 von 32 untersuchten Problemstellungen zu statistisch signifikant besseren Ergebnissen. In den anderen acht Fällen waren die Ergebnisse vergleichbar oder nicht statistisch signifikant schlechter als die der generischen Methodenvariante (nicht schlechter als 0,02 bezüglich gewichteter Jaccard-Koeffizienten). Dies suggeriert eine sichere Verwendung dieser Methodenvarianten in einer Reihe von Problemen beziehungsweise in einem Bereich von bestimmten Konditionen der Metriken. Alle Experimente wurden unter verallgemeinerbaren Bedingungen durchgeführt (zweifache Kreuzvalidierung und über 250 Millionen Segmentevaluierungen), wodurch auf eine generelle Nützlichkeit der Methoden geschlossen werden kann.

Die zweite vorgeschlagene Methodenvariante verwendet eine graphenbasierte, auf verbundenen Elementen basierende Segmentierung (mathematische Morphologie). Basierend auf dem Konzept von quasi-flachen Zonen, ist die eingeschränkte Konnektivität eine 
mögliche Variante einer solchen Segmentierung. Hier wird spektrale Unähnlichkeit als Konnektivitäts-relation definiert. Der Kern des Segmentierungsalgorithmus wird von einem zwei-dimensionalen Parameterraum gebildet, mit dem das Wachstum der Segmente beeinflusst werden kann. Dieser Ansatz erlaubt die Encodierung von zusätzlichen Attributen zur Schwellwertanwendung oder zum Verhindern von Segmentwachstum. Die Beeinflussung oder Beschränkung der Attribute bildet eine zusätzliche Komponente in einem erweiterten Design von Fitnesslandschaften für die stichprobenüberwachte Segmentgenerierung. Zudem können zusätzlich, ähnlich wie bei der oben präsentierten Methode, Datenabbildungsfunktionen integriert werden.

Daher wird eine Methode, bestehend aus den folgenden drei Komponenten vorgeschlagen: dem auf beschränkter Konnektivität basierenden Kernalgorithmus, der Datenabbildungskomponente sowie der Komponente für die Schwellwertbildung von Attributen. Sechs verschiedene Attribute (steigend/monoton) zur Beschreibung geometrischer und spektraler Charakteristiken werden hier untersucht. Diese Charakteristiken bilden im Kollektiv eine Komponente für die Schwellwertbildung. Es werden hier vier abgeleitete Methodenvarianten präsentiert, die aus vielfältigen Kombinationen von Komponenten bestehen $(\mathrm{CC}, \mathrm{CC}+\mathrm{Attr}$, $\mathrm{CC}+$ Map and CC + Attr + Map). Diese vier Varianten werden untereinander verglichen, um ihren Nutzen unter ähnlich umfangreichen Evaluierungsbedingungen, wie bei der Methodenvariante mit Datenabbildungsfunktion, aufzuzeigen.

Entgegen der ursprünglichen Vermutung, dass die Verwendung von Attributen nützlich ist, zeigen Kreuzvalidierungsergebnisse, dass dies keinen großen Mehrwert bringt. Im Gegensatz dazu hat der Ansatz der eingeschränkten Konnektivität, verbessert durch eine Auswahl von Datenabbildungsfunktionen, exzellente Resultate hervorgebracht. Dieser Ansatz, gefolgt von der komplexesten Methodenvariante unter Verwendung von Datenabbildung und Attributen, wurde von einem statistischen Ranking als die nützlichste Methode eingestuft. Die attributbasierte Komponente erfordert darüber hinaus zusätzliche Rechenzeit. Alle drei Komponenten weisen voneinander abhängige Parameter beziehungsweise Variablen auf. Wenngleich Attribute zur Schwellwertbildung in einigen Instanzen nützlich sind und in zwei Selektierungsproblemstellungen die besten Ergebnisse lieferten, sind diese dennoch mit Vorsicht in einer solchen Methode zu behandeln.

Die dritte vorgeschlagene Methodenvariante basiert auf der Verwendung der spektralen Information von bereitgestellten Referenzsegmenten. Es wird eine Methodenkomponente, die diese Daten nutzt, entworfen. Eine Auswahl von verschiedenen Varianten, bestehend aus unterschiedlichen Komponenten zur Anpassung der Daten mittels Klassifikation, wird hier vorgestellt. Die Erstellung einer Maske mit potentiell interessierenden Elementen erfolgt mittels Klassifikation der spektralen Daten (nicht-parametrisches, nicht-lineares Einzelklassen-Problem). Dieser Prozess repräsentiert einen Teil der SuchlandschaftKomponente und ist damit sowohl modifizierbar als auch justierbar. Die abgeleitete Maske wird für die Generierung von Beispielen einer synthetischen sekundären Klasse verwendet. Basierend auf dem daraus resultierenden Zwei-Klassen-Problem kann eine Wahrscheinlichkeitskarte generiert werden. Dieser Prozess stellt zudem auch einen Teil der 
Suchlandschaft-Komponente dar. In der vorliegenden Implementierung definieren fünf Parameter die Beschaffenheit der Wahrscheinlichkeitskarte.

Vier Methodenvarianten werden in dieser Arbeit vorgeschlagen und - unter Verwendung der Wahrscheinlichkeitsbilder für die Anpassung der Daten mit dem Ziel einer besseren Bildsegmentierung - untersucht. Die Varianten verfolgen verschiedene Ansätze zur Fusionierung oder Hybridisierung von Wahrscheinlichkeitsbild und ursprünglichen Eingangsdaten. Einige der Varianten beinhalten Parameter zur Kontrolle und Beeinflussung der Hybridisierung. Dies führt zu sehr modularen Methoden, wenngleich die Anzahl der Dimensionen einer Fitnesslandschaft hier erheblich ansteigen kann. Äquivalent zu den zuvor vorgestellten Methoden, werden auch hier ausgiebige Evaluierungen durchgeführt. Im vorliegenden Fall (nach Beseitigung einer problematischen empirischen Diskrepanzmetrik) wurden mit allen Varianten bessere Ergebnisse erzielt, als mit der generischen Variante der stichprobenüberwachten Segmentgenerierung. Insbesondere eine der Methoden (Hybrid:CP), die sehr moderate Interaktionen zwischen dem Wahrscheinlichkeitsbild und den ursprünglichen Eingangsdaten verwendet, liefert sehr vielversprechende Ergebnisse. Unglücklicherweise haben diese Methoden, verglichen mit der generischen Methodenformulierung, einen erheblich höheren Rechenaufwand zur Folge. Dieses Problem besteht aufgrund der Einbindung der Klassifikation (support vector machines) und könnte durch die Verwendung von Klassifikatoren mit schnelleren Trainingszeiten eingedämmt werden.

Die vorgestellten Methoden verwenden vielfältige Definitionen einer stichprobenüberwachten suchbasierten geographischen Bildsegmentierung unter Einbezug zusätzlicher Komponenten für eine erweiterte Fitnesslandschaft. Generell haben die meisten der vorgeschlagenen Varianten unter umfangreichen Evaluierungsbedingungen bessere Ergebnisse geliefert, als die ursprüngliche Formulierung. Diese Ergebnisse legen nahe, dass die vorgestellten Methodenentwürfe, basierend auf erweiterten Fitnesslandschaften beziehungsweise auf beispielüberwachten suchbasierten Ansätzen im Bereich der geographischen objektbasierten Bildanalyse, sehr vielversprechend sind und daher weiteren Untersuchungen unterzogen werden sollten. 


\section{ACKNOWLEDGEMENTS}

I should like to thank the following people and institutions that assisted me in some way or another during the course of this research project:

- Firstly I should like to note the GIONET project, with principal investigator Prof. Heiko and programme coordinator, Virginia. The financial support was greatly appreciated. My attending six international conferences (including IGARSS, GECCO and GEOBIA), four summer schools and conducting two secondments would not have been possible otherwise.

- All the 'Mitarbeiters' from the Department of Civil Crises Information and Georisks at the German Aerospace Centre in Oberpfaffenhofen. Talks with Hannes, Jens, Steffen, and office colleague, Linda, were especially entertaining and also productive.

- Fellow GIONET early stage researchers. The networking was a fruitful experience. Exchanges with James, Pedro, Maxim, Martyna, Bernard, Shailesh and Sybrand were always welcomed.

- Project Committee, Elisabeth, Stefan, Soren and Prof. Christiane, for interesting discussions - work and non-work related.

- The Schrafstätters from Gilching and the Kirsch family from Wessling - hospitable landlords who contributed to a wonderful three years for me in Bavaria.

- The Mayers from Roermoos, especially Corinna - thank you for all the support.

- Martyna for assisting in printing the dissertation in Jena.

- Elizabeth van Aswegen for proofreading the introduction and conclusion in this manuscript.

- Jens Kersten and Wolfgang Lück for translating the abstract into German.

- My family in South Africa needs a special thank you for accommodating me in the final months of writing. Aletta, Ettienne, Estelle and Bern, your support is greatly appreciated. 


\section{CONTENTS}

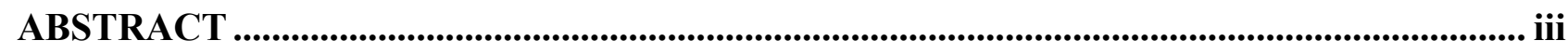

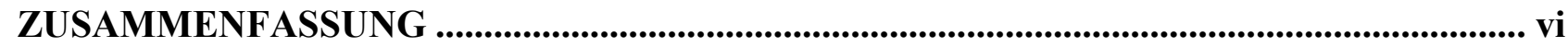

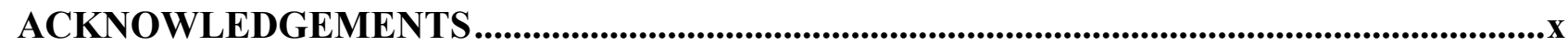

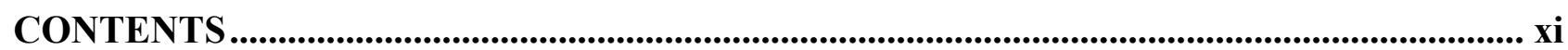

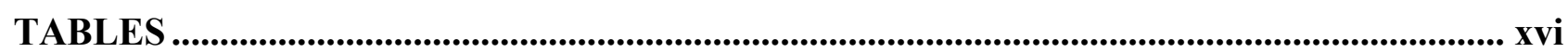

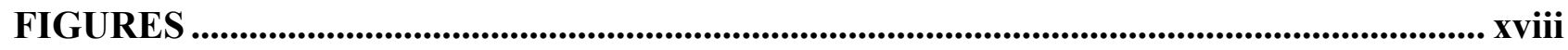

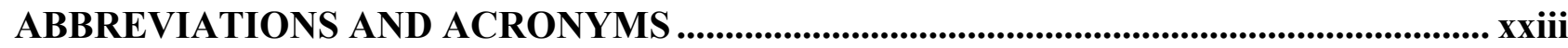

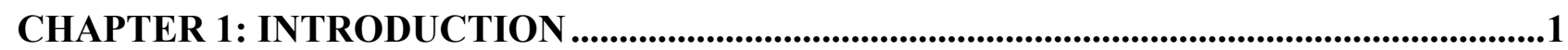

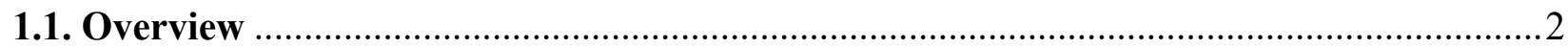

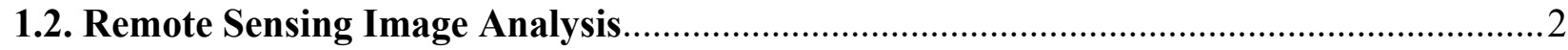

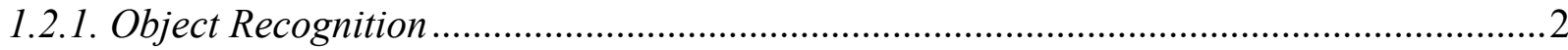

1.2.2. Very High Resolution Optical Remote Sensing Image Analysis ....................................... 3

1.2.3. Geographic Object-based Image Analysis ......................................................................

1.3. Automated Segmentation Algorithm Parameter Tuning .............................................5

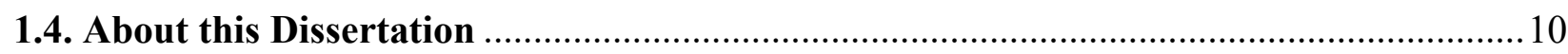

1.4.1. Expanded Fitness Landscapes for Geographic Sample Supervised Segment

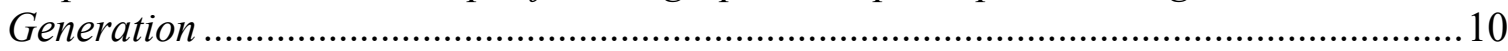

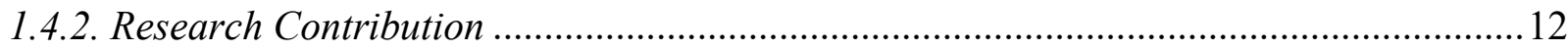

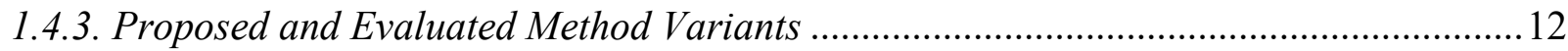

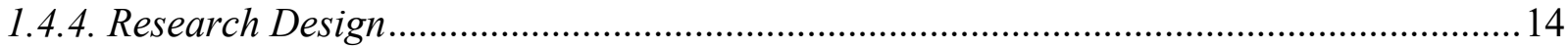

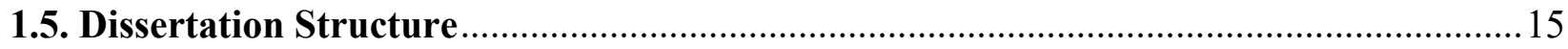

1.6. Publications Forming Part of this Dissertation .................................................... 15

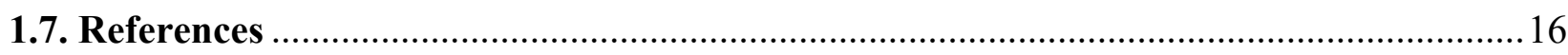

CHAPTER 2: SAMPLE SUPERVISED SEARCH-CENTRIC APPROACHES IN

GEOGRAPHIC OBJECT-BASED IMAGE ANALYSIS (GEOBIA): CONCEPTS, STATE-OF-THE-ART AND A FUTURE OUTLOOK..................................................................23

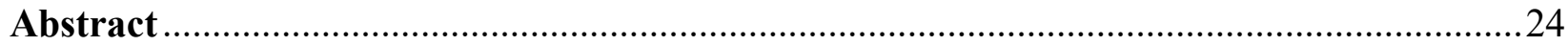

2.1. Search Centric Sample Supervised Image Analysis...................................................24

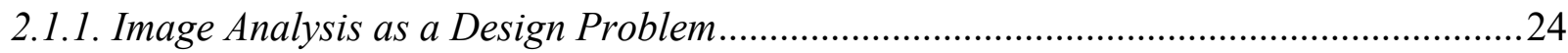

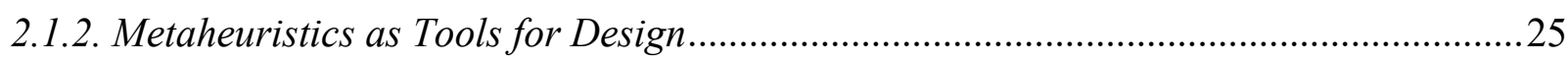

2.1.3. Granularity and Fitness Functions in Sample Supervised Search Centric Image

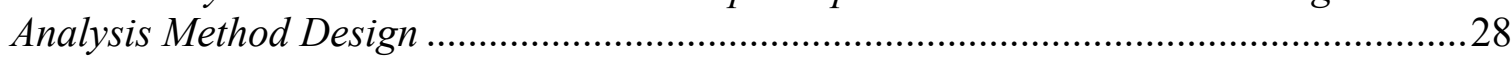

2.2. Approaches in Geographic Object-Based Image Analysis ..........................................29

2.3. Search Centric Sample Supervision within GEOBIA ….............................................31 
2.3.1. Automatic Segmentation Algorithm Parameter Tuning ................................................ 31

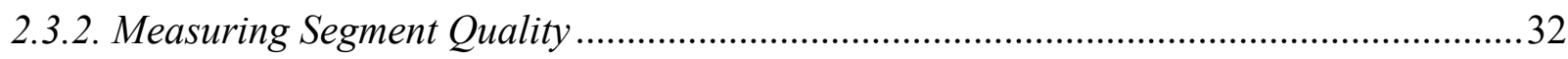

2.3.3. Advances in Search Centric Sample Supervised Segment Generation ............................33

2.4. Limitations of Search Centric Sample Supervised Segment Generation

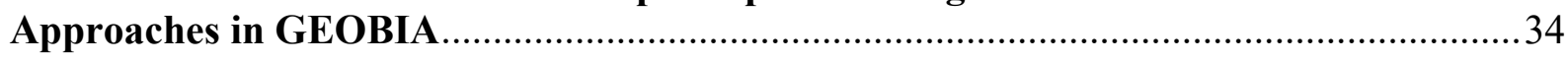

2.5. An Outlook on Search Centric Sample Supervision in GEOBIA Approaches -

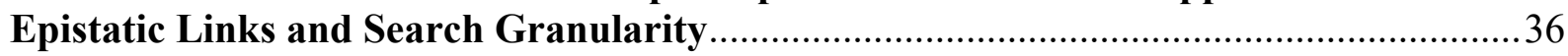

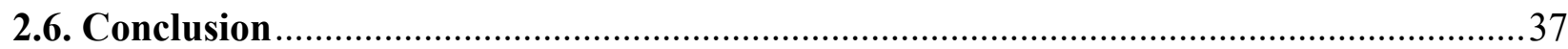

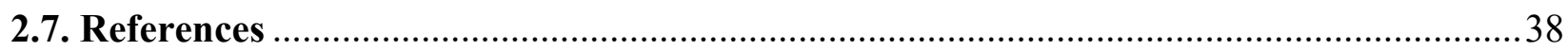

CHAPTER 3: DATA TRANSFORMATION FUNCTIONS FOR EXPANDED SEARCH SPACES IN GEOGRAPHIC SAMPLE SUPERVISED SEGMENT GENERATION ..............44

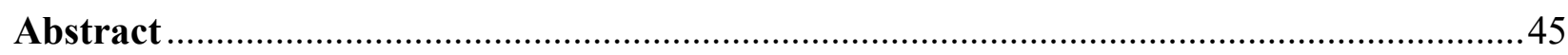

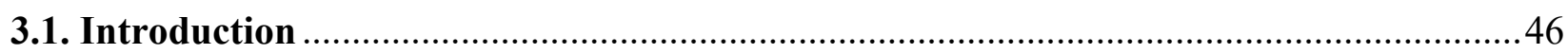

3.1.1. Semantic Segmentation in Geographic Object-Based Image Analysis ...........................46

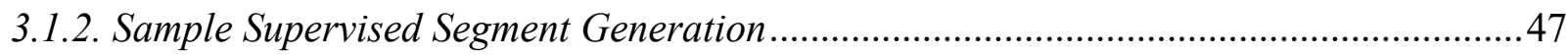

3.1.3. Integrative Approaches to Image Analysis ................................................................... 48

3.1.4. Combined Low- and Mid-Level Image Processing Optimization for Geographic Sample Supervised Segment Generation .................................................................... 48

3.2. Expanded Search Spaces in Sample Supervised Segment Generation ...........................49

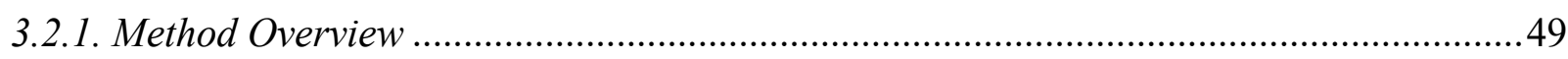

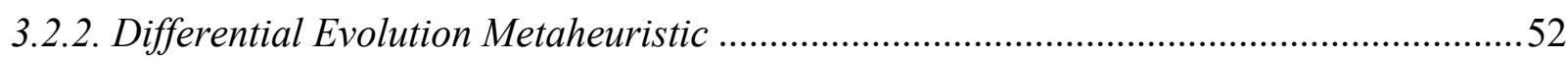

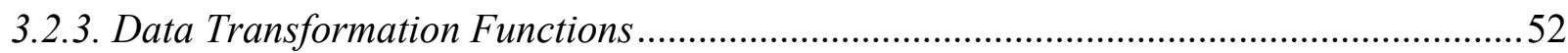

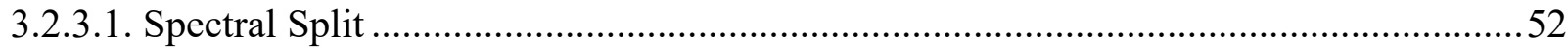

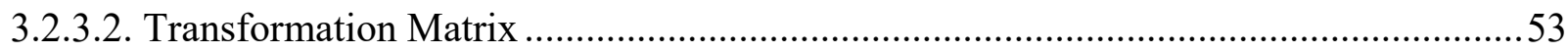

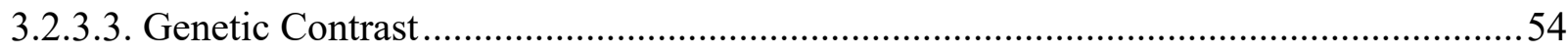

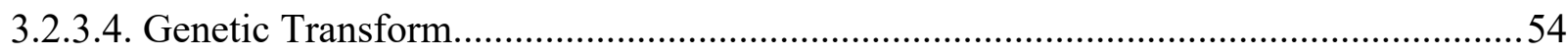

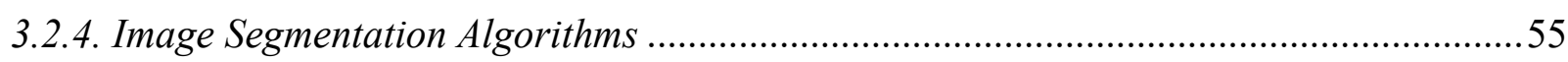

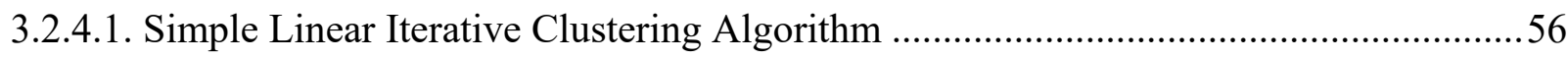

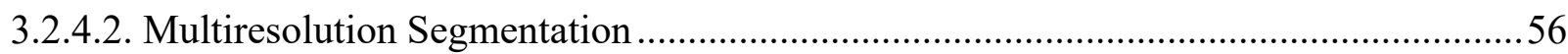

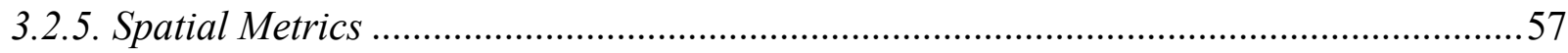

3.2.5.1. Reference Bounded Segments Booster ......................................................................58

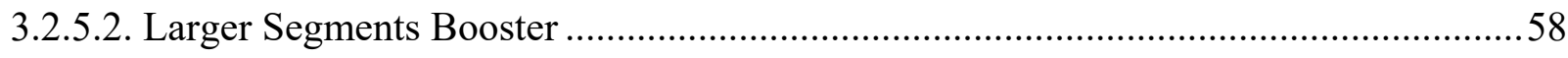

3.2.5.3. Partial and Directed Object-Level Consistency Error.................................................58

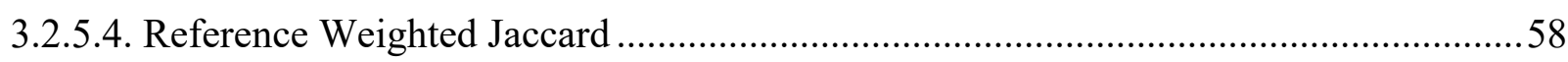

3.2.6. Examples of Data Transforms with Segments and Metric Scores ..................................58

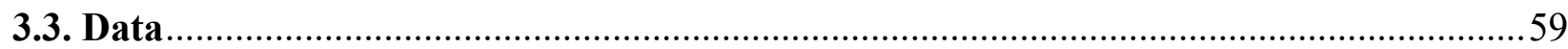

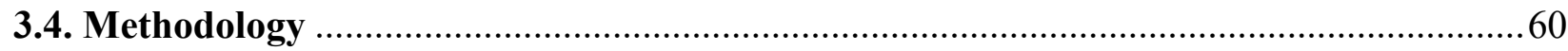

3.4.1. Data Transformation Functions for Expanded Search Spaces ........................................60 
3.4.2. Metaheuristic Evaluation and Convergence Behavior Profiling ....................................61

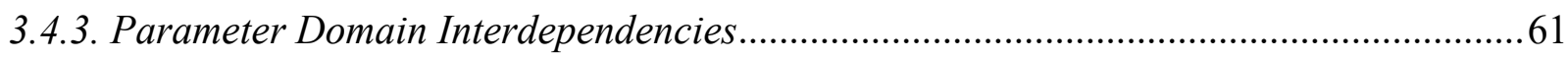

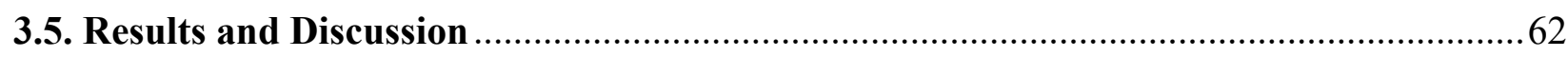

3.5.1. Data Transformation Functions for Expanded Search Spaces ......................................62

3.5.2. Metaheuristic Evaluation and Convergence Behavior Profiling ....................................65

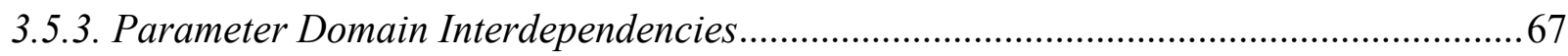

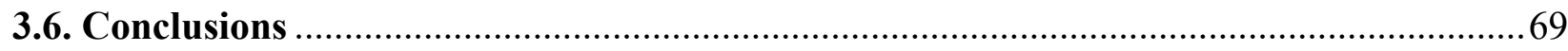

3.6.1. Potential of Expanded Search Spaces in Sample Supervised Segment Generation .........69

3.6.2. Methodological Shortcomings and Open Questions for Future Research ..................... 70

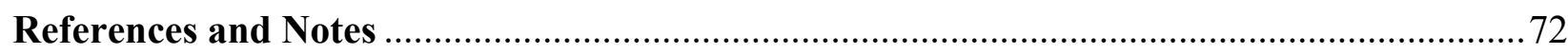

CHAPTER 4: ON ATTRIBUTE THRESHOLDING AND DATA MAPPING

FUNCTIONS IN A SUPERVISED CONNECTED COMPONENT SEGMENTATION

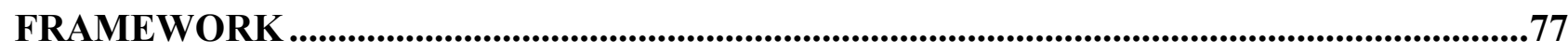

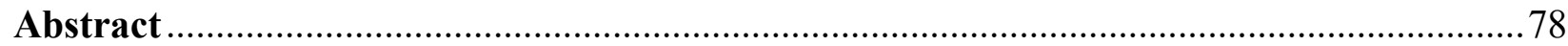

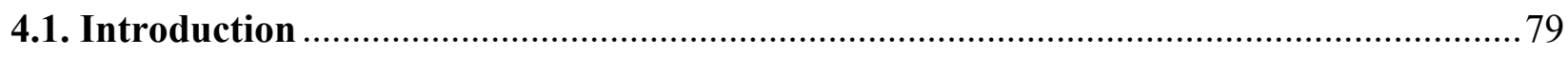

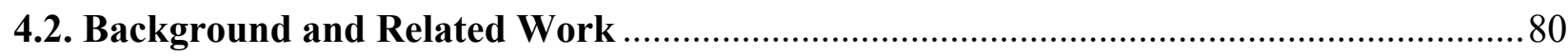

4.2.1. Graph-Based Connected Component Segmentation ....................................................... 80

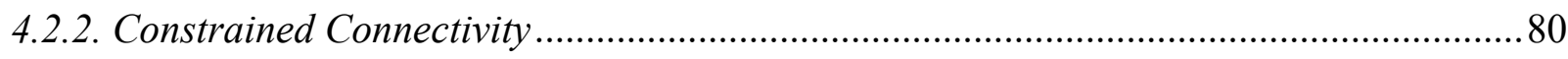

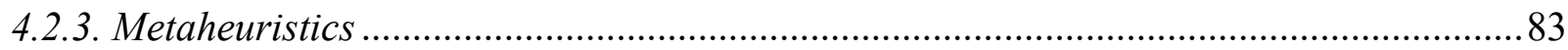

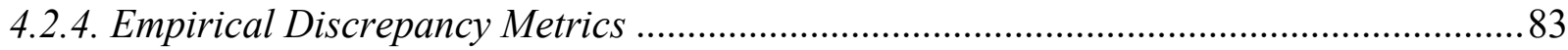

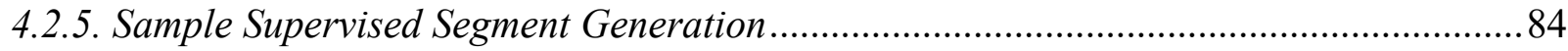

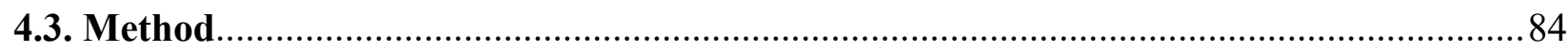

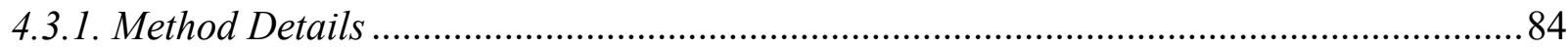

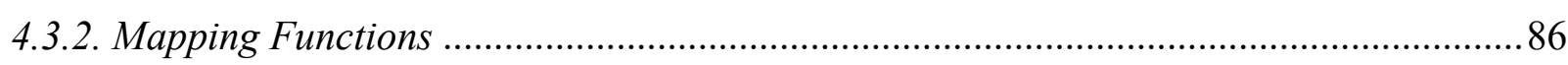

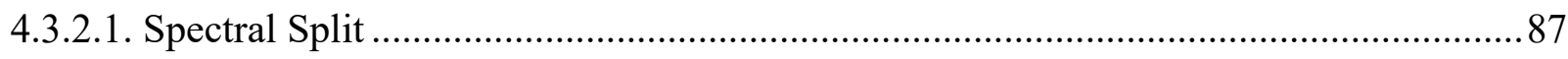

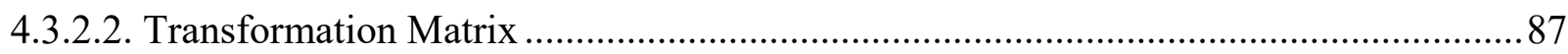

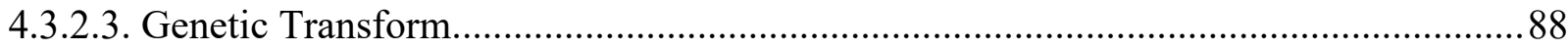

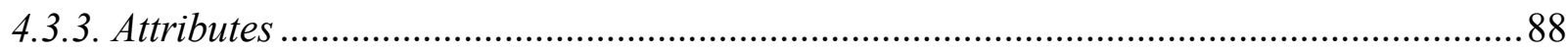

4.4. Data

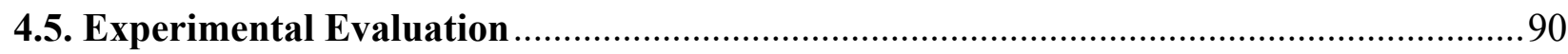

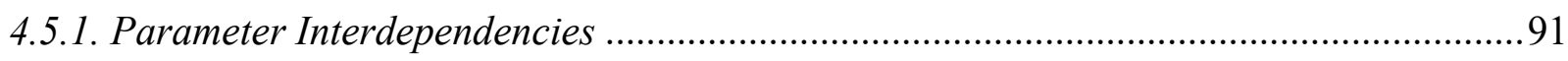

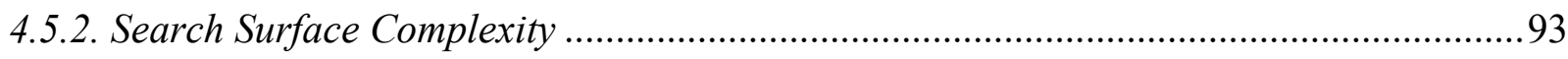

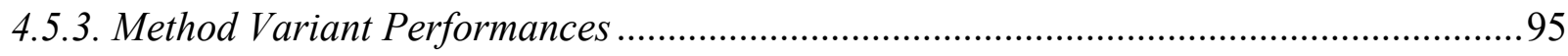

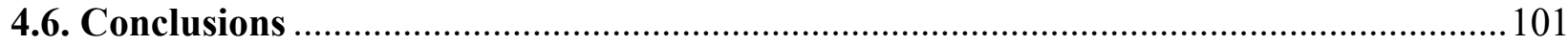

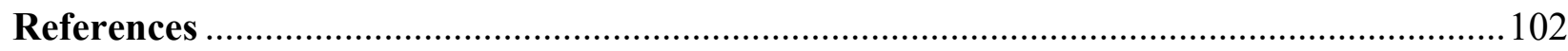




\section{CHAPTER 5: CLASSIFIER DIRECTED DATA HYBRIDIZATION FOR}

GEOGRAPHIC SAMPLE SUPERVISED SEGMENT GENERATION

Abstract 108

5.1. Introduction 109

5.2. Background and Related Work 110

5.3. Classifier Directed Data Hybridization 112

5.3.1. Exploiting Spectral Data Contained within Provided Reference Segments for Segment Generation... 112

5.3.2. Method Overview 113

5.3.3. Search Landscape: Sampling/Classification Sub-Component. 114

5.3.4. Search Landscape: Hybridization Sub-Component 116

5.3.4.1. Hybrid:EB (Exchange Band) 116

5.3.4.2. Hybrid:MA (Move to Average) 116

5.3.4.3. Hybrid:CP (Central Positions) 117

5.3.5. Search Landscape: Segmentation Sub-Component. 117

5.3.6. Metrics and Optimizers ..... 118

5.4. Data. 119

5.5. Experimental Design 120

5.5.1. Segment Quality Comparison and Method Ranking. 120

5.5.2. Search Process Characteristics 120

5.5.3. Parameter Interdependencies 121

5.5.4. Metaheuristic Viability

5.6. Results and Discussions 122

5.6.1. Segment Quality Comparison and Method Ranking.... 122

5.6.2. Search Process Characteristics 125

5.6.3. Parameter Interdependencies 128

5.6.4. Metaheuristic Viability 130

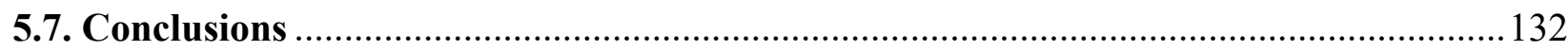

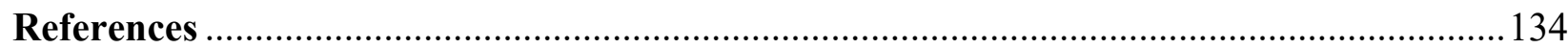

CHAPTER 6: CONCLUSIONS.

6.1. A Methodological Avenue within Geographic Sample Supervised Segment Generation 140

6.2. Additional Remarks on the Method Variants ............................................................. 141

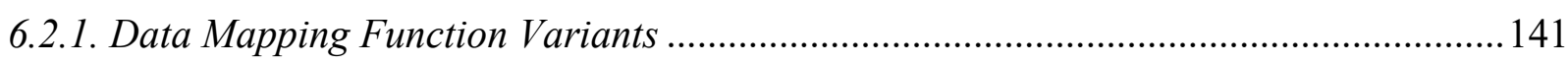

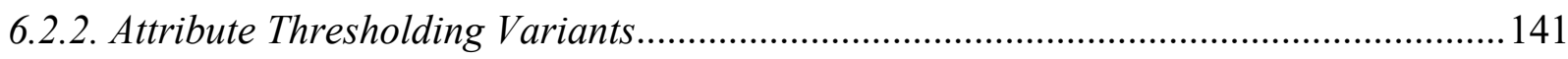

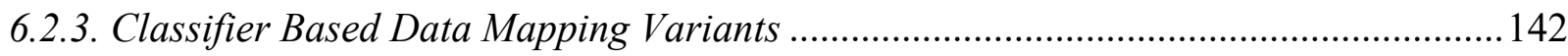


6.3.1. Metric Fitness Landscape Characteristics ................................................................... 142

6.3.2. Metrics Sensitive to Post-processes ............................................................................. 142

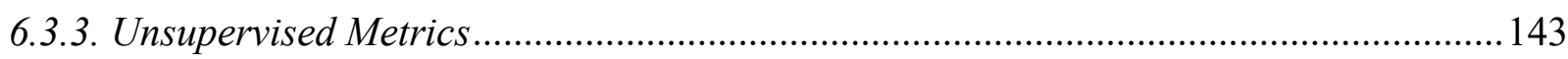

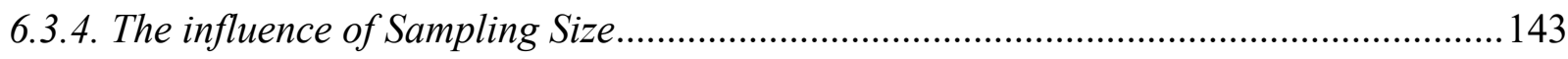

6.3.5. Incorporate Easy Segment Delineation Tools ................................................................ 143

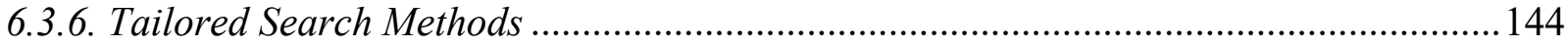

6.3.7. Correlation Between Metrics and Classifier Accuracy............................................... 144

6.3.8. A Comparison with Finer Grained Image Analysis Methods ....................................... 144

6.3.9. Classification Accuracy as Fitness Function ............................................................... 144

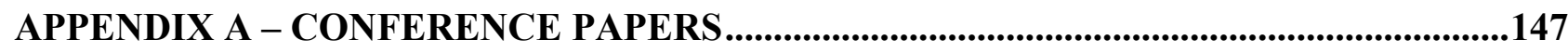

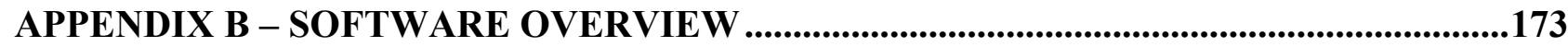

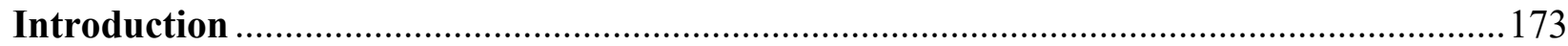

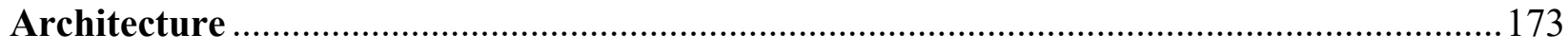

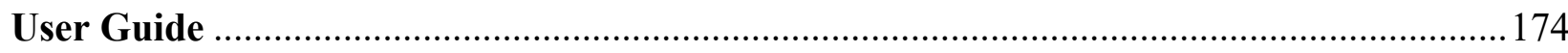

APPENDIX C - PARAMETER STANDARD DEVIATIONS ....................................................180

APPENDIX D - ALGORITHM DETAILS ..........................................................................182

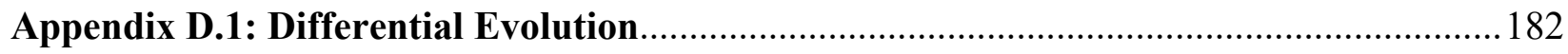

Appendix D.2: Multiresolution Segmentation .............................................................. 183

Appendix D.3: Simple Linear Iterative Clustering ........................................................ 185

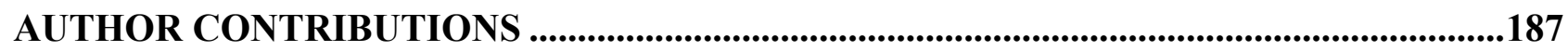

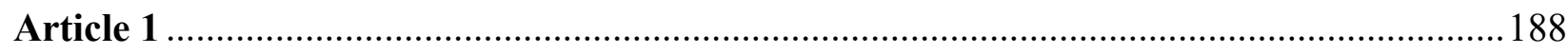

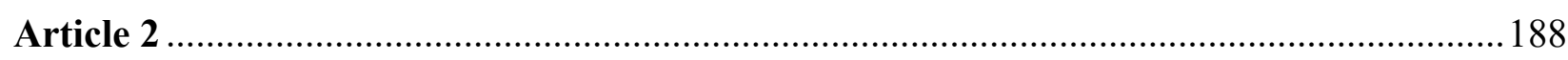

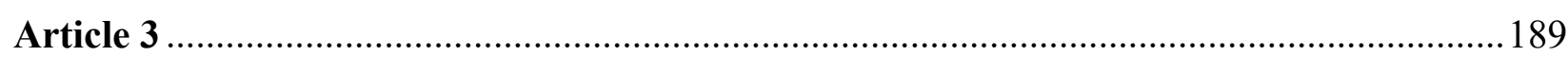

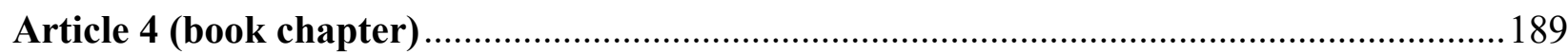

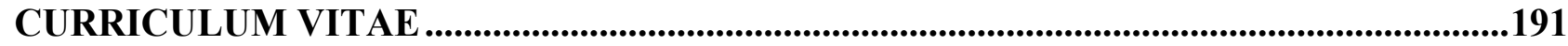

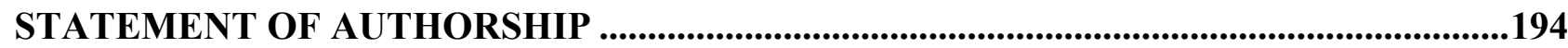




\section{TABLES}

Table 3.1. Dataset metadata, including the number of utilized reference segments............60

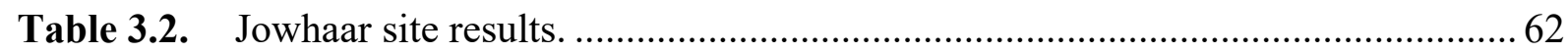

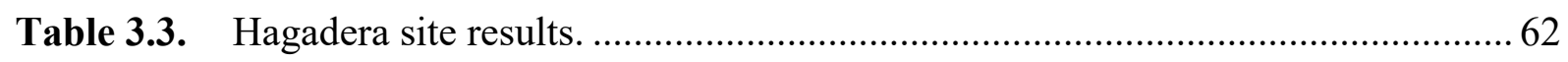

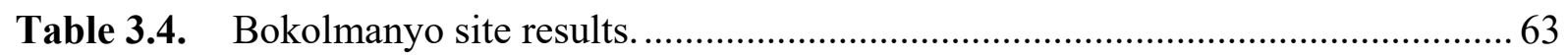

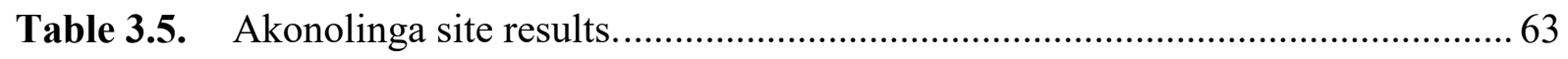

Table 3.6. Multiresolution Segmentation (MS) segmentation algorithm parameters obtained over different metric and data transformation function conditions for the Hagadera test site.

Table 3.7. SLIC segmentation algorithm parameters obtained over different metric and data transformation function conditions for the Bokolmanyo test site.

Table 3.8. Scale parameter values obtained (sorted) from the results depicted in Table 3.6 with MS segmentation, no transformation and the Reference Weighted Jaccard (RWJ) metric.

Table 3.9. Parameter domain interdependency test over 100 runs for the MS segmentation algorithm and Spectral Split transform on the Bokolmanyo site using the RWJ metric.

Table 4.1. The three empirical discrepancy metrics employed to measure the quality of generated segments against the provided reference segments.

Table 4.2. Implemented attributes for consideration in the context of CC segmentation, specifically in the CC + Attr and $\mathrm{CC}+\mathrm{Attr}+$ Map method variants.

Table 4.3. The datasets, with accompanying metadata, used for evaluating the method variants (adapted from [6]).

Table 4.4. Interdependency test of the method constituents for the Bokolmanyo problem. Note that all constituents affect one another. The mapping function affects all parameters most frequently.

Table 4.5. Interdependency test of the method constituents for the Jowhaar problem........ 92

Table 4.6. Interdependency test of the method constituents for the Hagadera problem...... 92

Table 4.7. Performance of the four search methods on the four method variants. In the simpler CC method variants (CC and CC + Attr), no benefit is noted from using more advanced search methods. In the case of the higher dimensional method variants $(\mathrm{CC}+$ Map and CC + Attr + Map), using an advanced search method becomes necessary.

Table 4.8. Method performance on the Bokolmanyo problem. Note the improved results with the CC + LIN and CC + Attr + LIN method variants under all metric conditions.

Table 4.9. Method performance on the Jowhaar problem. The method variant employing a data mapping function $(\mathrm{CC}+\mathrm{SS})$ performed the best under all metric conditions.

Table 4.10. Method performances on the Hagadera problem. The top performing method variant is metric dependent.

Table 4.11. Average computing times for experimental runs and resulting method parameters. Note the increased computing time of method variants employing attributes 
Table 5.1. The four spatial empirical discrepancy metrics used for segment evaluation, written using set theory notation

Table 5.2. Metadata of the datasets used

Table 5.3. Segmentation accuracies achieved in the Bokolmanyo problem instance. The shaded cells highlight the results of the best performing hybridization strategy.

Table 5.4. Segmentation accuracies achieved in the Jowhaar problem instance. The shaded cells highlight the results of the best performing hybridization strategy.

Table 5.5. Segmentation accuracies achieved in the Hagadera problem instance. The shaded cells highlight the results of the best performing hybridization strategy.

Table 5.6. Parameters generated for the Bokolmanyo problem instance........................... 128

Table 5.7. Parameters generated for the Hagadera problem instance............................... 128

Table 5.8. Parameters generated for the Jowhaar problem instance.

Table 5.9. RWJ metric scores using the four different search strategies in the Hagadera problem instance. The shaded cells highlight the results of the best performing search method. 


\section{FIGURES}

Figure 1.1. [14] Two generalised approaches for image analysis found within GEOBIA. Progressing from image objects to geographic objects (classification) may involve multiple runs of a given segmentation algorithm (Figure 1.1(a)), hierarchical segmentation (Figure 1.1(b)) and various approaches modifying segments based on expert, hierarchical or contextual knowledge.

Figure 1.2. An example image analysis task where the aim could be to identify and characterise all structures. Seven tents were digitised and presented to a generic sample supervised segment generation method as reference segments.

Figure 1.3. [63] The general architecture of the generic variant of sample supervised segment generation as found in $[59,62,66]$. .

Figure 1.4. [61] An abstract segment quality evaluation scenario, where a given metric measures segment quality and provides the output to the sample supervised segment generation method.

Figure 1.5. An example two-dimensional parameter slice (three dimensions in this example), or fitness landscape, generated by RWJ. Note the multimodal nature (multiple local minima).

Figure 1.6. Two benchmark search problems where points depict positions visited by a given optimiser [71], illustrating an "easy" (a) fitness landscape and a "difficult" fitness landscape (b).

Figure 1.7. Basic classification results after running the generic variant of sample supervised segment generation and conducting basic classification (one-class SVM). Note the bad geometry of the segments related to the tents.

Figure 1.8. Enlargement over a part of Figure 1.2 to illustrate the difficult nature of the image analysis problem. Elements of interest (tents) are spectrally diverse, not homogeneous, not always properly separated from one another, and have soft transitions with their surroundings.

Figure 1.9. Expanded fitness landscapes in sample supervised segment generation. The shaded cells (grey) illustrate additional constituents that may be added to the general method (white cells). The three processing steps thus combine to form an expanded fitness landscape.

Figure 1.10. [61] Expanded fitness landscape via data mapping or transformation functions. . 13

Figure 1.11. [86] Sample supervised segment generation incorporating the $\mathrm{CC}$ algorithm, data mapping functions and thresholdable attributes.

Figure 1.12. [88] A sample supervised segment generation method design incorporating classification processes.

Figure 2.1. Generalised architecture of simple population based metaheuristics............. 26

Figure 2.2. An arbitrary search landscape, with positions visited by a metaheuristic during the search process denoted with red dots.

Figure 2.3. Fitness traces generated by two different search methods for an arbitrary design problem in an image analysis context

Figure 2.4. A simple segmentation problem where spectrally and geometrically similar elements of interest are segmented with a single segment layer. 
Figure 2.5. Generalised architecture of a search centric sample supervised segment generation approach.

Figure 2.6. An example mapping scenario where such an automatic segmentation algorithm parameter tuning process may form part of the image analysis workflow.

Figure 2.7. A representation of area overlap. Empirical discrepancy measures or spatial metrics could measure the amount of spatial overlap between the reference $(R)$ and a generated (S) segment.

Figure 2.8. Three segmentation evaluation examples using the RWJ metric evaluating the mismatch between reference (red line) and generated (green lines) segments. 33

Figure 2.9. A segmentation scenario where the object of interest could be buildings of vastly different spectral and geometric characteristics.

Figure 2.10. An arbitrary search centric image analysis method executed on a artificial problem (a) considering pixel-based classification processes (b, c) and their interaction with data transformation and segmentation processes (d).

Figure 3.1. The method architecture for sample supervised segment generation with added data transformation functions.

Figure 3.2. An example encoding of a parameter set traversed by a given search algorithm, highlighting the creation of a joint set from the segmentation algorithm and a low-level image processing function.

Figure 3.3. Two dimensions (band position 1 and scale parameters) of the seven dimensional search space of the parameter set depicted in Figure 3.2 (average of 15 reference segments) using the Reference Weighted Jaccard (RWJ) metric (Section 3.2.5).

Figure 3.4. An example user interface for the search centric sample supervised segment generation approach described in this work.

Figure 3.5. Output imagery from four data transformation functions tested in this work as part of a combined search space: (a) No Transformation, (b) Spectral Split, (c) Transformation Matrix, (d) Genetic Contrast, (e) Genetic Transform........ 53

Figure 3.6. Four parameterized transformation functions, weighted to constitute the Genetic Transform function 56

Figure 3.7. An abstract segmentation evaluation scenario with the reference segment denoted by $\mathrm{R}$, the generated segments by $\mathrm{Si}$ and the generated segment with the largest overlap with $\mathrm{R}$ as $\mathrm{S}$.

Figure 3.8. $\quad$ RWJ metric scores on a single object (house) of interest delineated by a bold red line. The Multiresolution Segmentation algorithm and Spectral Split transformation function were employed.

Figure 3.9. The four datasets used in this study, entitled (a) Jowhaar, (b) Hagadera, (c) Bokolmanyo, (d) Akonolinga. Dataset details are given in Table 3.1

Figure 3.10. Averaged RWJ metric scores (lower is better) for SLIC segmentation on the four sites using the five different transformation function strategies.

Figure 3.11. Example of best segmentation results achieved on (a) original image and (b) an image transformed with the Genetic Transform function for the Bokolmanyo site using the MS segmentation algorithm and RWJ metric. 
Figure 3.12. Example of best segmentation results achieved on (a) the original image and (b) an image transformed with the Transformation Matrix for the Hagadera site using the MS segmentation algorithm and RBSB metric. .65

Figure 3.13. Fitness traces for three search methods on the Bokolmanyo site (MS segmentation) using the (a) No Transform, (b) Transformation Matrix, (c) Spectral Split and (d) Genetic Transform transformation strategies. The x-axes delineate search iterations. The y-axes delineate RWJ evaluation scores. 65

Figure 3.14. Fitness traces for three search methods on the Jowhaar site (SLIC segmentation) using the (a) No Transform, (b) Transformation Matrix, (c) Spectral Split and (d) Genetic Transform transformation strategies. The x-axes delineate search iterations. The y-axes delineate RWJ evaluation scores........ 66

Figure 3.15. Fitness traces for the two tested segmentation algorithms (SLIC and MS) on the Jowhaar site using the different transformation function strategies. The $\mathrm{x}$ axes delineate search iterations. The y-axes delineate RWJ evaluation scores.67

Figure 4.1. An abstract image, segmented with the $\alpha \mathrm{w}-\mathrm{CC}$ method illustrating its general characteristics, with $(0,0)-\mathrm{CC}$ shown in (a), $(1,1)-\mathrm{CC}$ in (b), (1,2)-CC in (c), and (2,3)-CC in (d).

Figure 4.2. An image subset (a) segmented with ( $\alpha \mathrm{w}-\mathrm{CC})$ to show its common characteristics on real imagery, with the local and global range parameters set to 25 and 75 (b), 25 and 75 with a region growing filter (c), and to 50 and 200 (d), respectively. The red polyline indicates an example element a user might be interested in.

Figure 4.3. The architecture of the sample supervised segment generation method incorporating data mapping functions and attribute thresholding [3]. IEEEC 2013. Reprinted, with permission, from [3].

Figure 4.4. An example of an 11-dimensional parameter set traversed by the CC + Map + Attr full method variant. Example parameters within each constituent are written vertically.

Figure 4.5. Example output of the three used mapping functions on an arbitrary test image (a). Parameters were assigned random values. The red polyline denotes an example element of interest; (b) shows output of the SS function (note the creation of sharp gradients); (c) shows output from the transformation matrix, while (d) shows the output from the GT function. Note the non-linear stretch of the output from the GT function.

Figure 4.6. An image subset segmented with the local range parameter set to 50 and the global range parameter set to 200. Additional constraining attributes are introduced, specifically area, with a value of 800 (a) and $\mathrm{CH} 1$ (b) with a value of 300 .

Figure 4.7. The three image analysis tasks defined for evaluating the method variants, namely, thematically correctly segmenting tents in the Bokolmanyo problem (a) and metal-roofed structures in the Jowhaar (b) and Hagadera (c) problems.

Figure 4.8. Two-dimensional parameter plots, or search surfaces, demonstrating parameter interactions between method constituents: (a) illustrates the interaction of the 
alpha parameter from the $\mathrm{CC}$ constituent and that of a mapping function parameter, while (b) shows the interaction of alpha with the $\mathrm{CH} 1$ attribute.... 93

Figure 4.9. Search method profiles for the four method variants, namely $\mathrm{CC}$ (a), CC + Attr (b), CC + Map (c), and CC + Attr + Map (d). Note the increased performance of DE and PSO when considering the CC + Map and CC + Attr + Map method variants 95

Figure 4.10. Exemplar optimal segmentation results focused on a random reference segment. The rows depict the CC, CC + Attr, CC + Map, and CC + Attr + Map method variants respectively (in order). The columns denote the three problems, Bokolmanyo, Jowhaar, and Hagadera (in order). (a) RWJ: 0.574; (b) RWJ: 0.524; (c) RWJ: 0.787; (d) RWJ:0.683; (e) RWJ: 0.524; (f) RWJ: 0.806; (g) RWJ: 0.104; (h) RWJ: 0.506; (i) RWJ: 0.787; (j) RWJ: 0.063; (k) RWJ: 0.437 ; (1) RWJ: 0.549.

Figure 4.11. Search method profiles for the different problems under different metric conditions. Note that for the simpler Bokolmanyo problem near-optimal results are achieved relatively early on in the search process. In the more complex problems, the methods need substantially more iterations in finding the achievable optimal parameter set.

Figure 4.12. Friedman rank test with a Nemenyi post hoc test conducted on results from Tables 4.8-10. Confidence interval is set to 95\%. A Critical Difference (CD) of 0.349 is generated (ranking). All method variants deliver statistically significant different results. Generally speaking, the $\mathrm{CC}+$ Map method variant was found most useful.

Figure 5.1. Architecture of the generic formulation of sample supervised segment generation [39].

Figure 5.2. The rationale behind the proposed method. Segment geometry is commonly provided via digitizing and used to drive a parameter search process (Arrow A) for segment generation. Reference segment spectral content (Arrow B), which is implicitly provided, is queried to influence a data transformation (Arrow C) affecting segment generation.

Figure 5.3. Architecture of the variant of sample supervised segment generation incorporating classifier directed data hybridization [30].

Figure 5.4. An example parameter set, forming the search landscape with interdependent real-valued parameter domains [30].

Figure 5.5. Image processing conducted to derive a probability image. (a) Illustrates a subset with a delineated reference segment; (b) the masking and synthetic sampling procedure and (c) the generated probability image

Figure 5.6. The three variants of data hybridization investigated. (a) Illustrates a hybrid image generated via the (a) band replacement strategy (Section 5.3.4.1); the (b) move to average strategy (Section 5.3.4.2) and (c) the move to central positions strategy (Section 5.3.4.3).

Figure 5.7. Segmented subsets, generated with the MS algorithm using the (a) original image, and a (b) Hybrid:CP image and segments generated with SLIC using a (c) probability image. 
Figure 5.8. The datasets used for method evaluation, namely (a) Bokolmanyo; (b) Jowhaar and (c) Hagadera. The blue polygons in the enlarged subsets represent digitized reference segments.

Figure 5.9. Segmentation results achieved with the Jowhaar problem instance (MS) using the RWJ metric. The red polyline delineate one of the twenty reference segments (a) provided by a user; (b) Shows results with the generic formulation; (c) using the probability image and the (d) Hybrid:EB; (e) Hybrid:MA and (f) Hybrid:CP strategies. RWJ metric scores for this specific structure are also given.

Figure 5.10. Nemenyi post hoc test performed after a Friedman test, ranking the different methods over all metric and problem instances. Horizontal lines indicate the Critical Differences (CD).

Figure 5.11. The Nemenyi post hoc test, with the RBSB metric omitted. Horizontal lines indicate the Critical Differences (CD).

Figure 5.12. Fitness traces for the Bokolmanyo problem instance................................... 126

Figure 5.13. Fitness traces for the Jowhaar problem instance. ...................................... 126

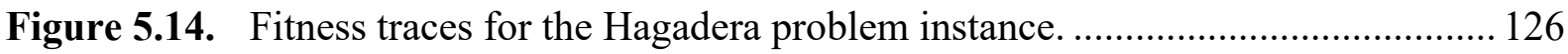

Figure 5.15. Search iteration execution time profiling for the Bokolmanyo problem instance.

Figure 5.16. Search iteration execution time profiling for the Jowhaar problem instance. 127

Figure 5.17. Search landscape slice with the Scale and CP2 parameter of the Hybrid:CP method variant.

Figure 5.18. Search landscape slice with the Scale and two-class SVM C parameter of the Hybrid:CP method variant

Figure 5.19. Search landscape slice with the Scale and probability weighting parameter of the Hybrid:CP method variant.

Figure 5.20. Fitness traces using different search methods for the Hagadera problem instance. Each subfigure shows results using a different method variant. 


\section{ABBREVIATIONS AND ACRONYMS}

ATTR Attribute

CC Constrained Connectivity

CD Critical Difference

$\mathrm{CH} \quad$ Grey Level Difference Histogram

CIELAB Commission Internationale de l'éclairage LAB (Lightness \& a, b)

CMP Compactness

CP Central Position

CR Crossover Constant

DE Differential Evolution

EC European Commission

ESA European Space Agency

EUSI European Space Imaging

EVI Enhanced Vegetation Index

GA Genetic Algorithm

GECCO Genetic and Evolutionary Computation Conference

GEOBIA Geographic Object Based Image Analysis

Geo-ND Geographic Object Novelty Detector

GIS Geographic Information System

GLCM Grey Level Co-Occurrence Matrix

GMES Global Monitoring for Environment and Security

GT Genetic Transform

GUI Graphical User Interface

HC Hillclimber

HD Heuristic Domain

HSI Hue, Saturation and Intensity

HYBRID:CP Hybrid Central Positions

HYBRID:EB Hybrid Exchange Band

HYBRID:MA Hybrid Move To Average

IDE Integrated Development Environment

IDP Internally Displaced Person

IGARSS International Geosciences and Remote Sensing Symposium

ISI Institute for Scientific Information

KNN K-Nearest Neighbour 
LSB Larger Segments Booster

MAP Mapping Function

MS Multiresolution Segmentation

NDVI Normalised Difference Vegetation Index

OCE Object-Level Consistency Error

OpenCV Open Computer Vision Library

PD_OCE Partial and Directed Object-Level Consistency Error

PSO Particle Swarm Optimisation

Qt "Cute" Application Framework

R Reference Segment

RBF Radial Basis Function

RBSB Reference Bounded Segments Booster

RGB Red Green Blue

RND Random (Search)

RS Remote Sensing

RWJ Reference Weighted Jaccard

S Generated Segment

SD Spectral Domain

SLIC Simple Linear Iterative Clustering

SMT Smoothness

SPT Segmentation Parameter Tuner

SS Spectral Split

SVM Support Vector Machine

TIFF Tagged Image File Format

VHR Very High Resolution 


\title{
CHAPTER 1: INTRODUCTION
}

\author{
Overview \\ Remote Sensing Image Analysis \\ Automated Segmentation Algorithm Parameter Tuning \\ About this Dissertation \\ Dissertation Structure \\ Publications Forming Part of this Dissertation
}




\subsection{Overview}

This dissertation elaborates upon methodology within the context of sample supervised search centric geographic image segmentation. In such a method, segments are automatically generated based on example reference segments. Expanded fitness landscapes are proposed to improve segment quality and a range of method variants are presented in this vein.

In this chapter, a section is presented (Section 1.2) to set the context of this work. This leads to the description of a specific remote sensing image analysis methodology and related problem formulation in Section 1.3. The contribution of this work is presented in Section 1.4. This is followed by a description of the dissertation structure (Section 1.5) and a list of publications and secondary material submitted as part of this dissertation (Section 1.6).

\subsection{Remote Sensing Image Analysis}

\subsubsection{Object Recognition}

Digital image processing and image analysis methodology have garnered great interest in the last four decades within scientific disciplines that may possibly be labelled, collectively, as the image analysis sciences. Image analysis encompasses the extraction of information from imagery, or the provision of information, via the process of image processing. Varied approaches exist and new ones are continuously being proposed to extract information from imagery, thereby pushing the boundaries of efficiency and accuracy. The applicability of a method is strongly dependent on the nature of the data and the desired information product.

Although various general works exist, echoing these broad sentiments and detailing the various variants of image analysis, e.g. [1-3], a series of short course materials summarises the main types of object recognition succinctly [4]. Object recognition implies identifying and labelling an image, or sub-parts of an image and is the type of image analysis considered in this dissertation. The authors of [4] discuss four broad types of object recognition, namely bag-of-words models, parts-based models, discriminative methods and concurrent segmentation and recognition. The bag-of-words approach entails the creation of histograms of small elementary image parts and their subsequent classification. Similarly, a part-based approach analyses elementary image parts, but also considers their spatial distributions, or context.

Discriminative methods entail the use of classification, with an image partitioned into smaller parts or overlapping windows. A set of rich features or attributes may be calculated for these parts and a given classifier may be tasked to judge if a part contains, or is, a certain element of interest (e.g. an example from within remote sensing [5]). Segmentation and recognition entail the partitioning of an image, with the aim of generating and subsequently identifying semantically correct image partitions. These broad method categories describe general processes, with specific methods combining aspects of these processes, or defining interacting processes, being common [6-11]. Generally, there is an underlying research theme in the image analysis sciences of improving the quality of results, among other research goals. 


\subsubsection{Very High Resolution Optical Remote Sensing Image Analysis}

Within the discipline of remote sensing, there also exists an extensive body of work focusing on methodological aspects of image analysis. Methodology, as considered here, implies the creation of information products, or maps, derived from satellite and airborne imagery of the earth's surface. Such imagery has unique characteristics as opposed to imagery or data encountered within other imaging disciplines. Optical remote sensing image analysis methodology has undergone a well reported and significant shift since the turn of this century, mainly owing to the proliferation of commercially available Very High Resolution (VHR) optical imagery [12-16] and subsequent challenges to and potential benefits of its analysis.

Traditionally, optical remote sensing image analysis places firmer emphasis on concepts of context and spatial aggregation, loosely speaking, using notions of both parts-based models and simultaneous segmentation and recognition. Image segmentation is a tool well suited to assist in the analysis of VHR optical data $[13,17,18]$. This may be due to the typical scenario of scene objects consisting of multiple resolution cells, or pixels, within VHR optical data, and to the presence of common spectral and thematic relations of scene objects [14].

The breadth of literature on image segmentation is extensive [19-21], with remote sensing also displaying various novel and adapted methodologies [13,18]. Followed approaches within remote sensing include, but are not limited to, region merging variants (e.g. the well-known Multiresolution Segmentation (MS) [22]), region growing segmentation [23], watershed-based approaches [17] and mathematical morphology and graph-based segmentation [24,25]. Spectral, geometric and textural properties are commonly considered as homogeneity predicates [26]. See [27] for an overview of a selection of segmentation algorithms used in practice within remote sensing. It should be noted that other image processing could also be considered to conduct simultaneous object segmentation and recognition, including Cartesian genetic programming [28], cellular automata [29] and various approaches from mathematical morphology [30] and computer vision [31].

Various aspects need consideration when utilising segmentation as a primary tool in VHR optical image analysis. Examples include notions of hierarchical segmentation [12,32], segment quality [3335], the notion of semantically meaningful image segments [36], the integration of context information [37], classification/machine learning approaches to be used [38,39], feature descriptors to be considered [40], data representation [41], computational costs [42] and the general design of an automated or manual method or workflow [37,43]. An academic sub-discipline has evolved around the broad theme of simultaneous segmentation and recognition within remote sensing, in part to address many of these considerations, namely Geographic Object-Based Image Analysis (GEOBIA) [14].

\subsubsection{Geographic Object-based Image Analysis}

GEOBIA defines a methodological avenue or a style of image analysis approaches within remote sensing [14-16,36,37,43-45]. GEOBIA strives to address the general themes of deriving information products faster or more autonomously, and generating more accurate products. At the core of GEOBIA is the creation of initial image partitions, most commonly through image segmentation [14], and the subsequent aim of progressing to thematically accurate land-cover information. Cognitive or user- 
driven method building is common (rule-set development) [14,37]. A distinction may be made between thematically meaningful segments/geographic objects and general image objects [36]. How to progress from image objects to geographic objects may also be considered a research theme within GEOBIA. It should be noted that GEOBIA might be considered a specific viewpoint or community and that works involving simultaneous segmentation and recognition within remote sensing may not necessarily fall within its definitions or aims (see [14] for an indication of the research agenda of GEOBIA).

Various approaches exist within GEOBIA to ultimately generate geographic objects. Generally, it is considered that a single level of image segmentation and classification of segments is insufficient for most mapping tasks. This is mainly due to variations in thematic and spectral correlations of landcover elements and the subsequent inability of a given segmentation algorithm to generate the desired semantic segments. Additional procedures would be needed to progress to semantic segments/geographic objects. Figure 1.1 [14] illustrates two such general approaches, advocated and commonly seen in practice [37]. In Figure 1.1(a), a workflow is shown where multiple sets of segments are generated (different algorithms/parameterisations) and iteratively refined via various image processing and classification tasks. Figure 1.1(b) is similar, with a focus on a hierarchical segmentation, and additionally using information present in the hierarchy to progress to a final classification. Note that these processes are user driven.
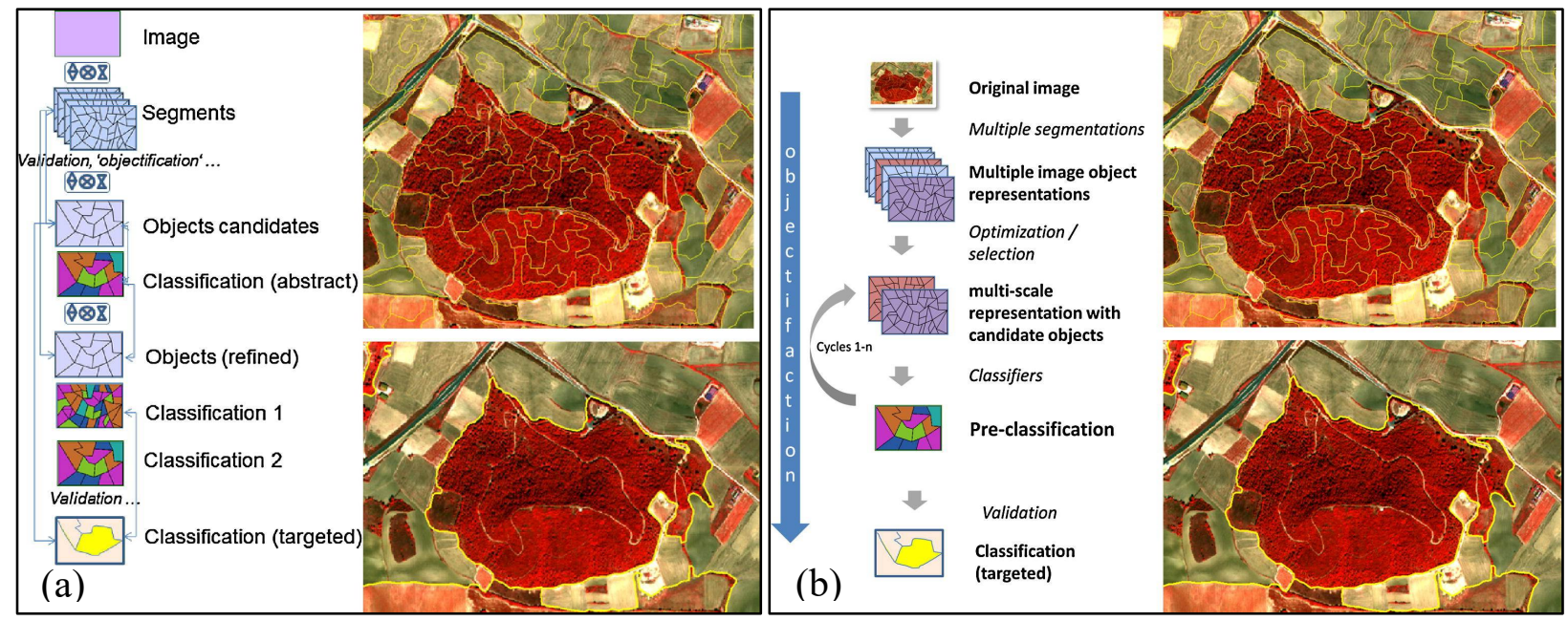

Figure 1.1. [14] Two generalised approaches for image analysis found within GEOBIA. Progressing from image objects to geographic objects (classification) may involve multiple runs of a given segmentation algorithm (Figure 1.1(a)), hierarchical segmentation (Figure 1.1(b)) and various approaches modifying segments based on expert, hierarchical or contextual knowledge.

Although deriving semantically correct image segments would typically not initially be possible (the reason for the approaches such as in Figure 1.1), it is still aimed at and is an area of active research within remote sensing and beyond [14,23,46-52]. Generally, the closer a partitioning approaches the desired final result, the fewer subsequent processes need to be invoked or designed (e.g. via rule-sets or other automated procedures). Image segment quality is thus an important and well-studied measure within such approaches [27,33-35,53-55]. 
Improving segment quality (automatically) has substantial benefits in terms of automating methods and creating more accurate information products. This ultimately contributes to the greater theme within remote sensing of providing geo-spatial intelligence. A selection of automatic techniques exists to assist in the process of generating thematically correct image segments, broadly categorised based on their need for user interaction. They may be employed in various stages of the general approaches depicted in Figure 1.1. These techniques find their origin in other imaging disciplines, where similar problems have been encountered. Unsupervised methods attempt to define an appropriate segmentation automatically based on image-wide statistics [12,54,56-58], either in the context of a single-scale representation or a multi-scale representation (e.g. Figure 1.1(b)). A criticism of such approaches is their insensitivity to targeted elements of interest.

Another approach attempts to generate segments based on the provision of a selection of exemplar, template or reference segments [46,52,53,59-62]. The geometry and other properties of the provided reference segments are used to drive a search or construction process for quality segmentation. Supervised segment measures, or rather empirical discrepancy metrics [35], are used to judge segment quality and drive the search or construction process. Such a general approach has the advantage of being able to tailor segmentation to a specific element of interest and removes the guesswork and labour that would be needed by an image analyst otherwise. This approach may be called supervised segmentation or automated segmentation algorithm parameter tuning. Here, more holistically, it is referred to as a sample supervised search centric approach within geographic object-based image analysis [63]. It is the general method considered, and elaborated upon, in this dissertation.

\subsection{Automated Segmentation Algorithm Parameter Tuning}

The general supervised segmentation algorithm parameter tuning approach forms part of a family of methods found at the intersection of the image analysis sciences and the sub-discipline of computer science focused on efficient search methods (e.g. evolutionary computation, metaheuristics). The authors of [64] (Chapter 1) present an overview of ways in which efficient search methods may be employed within image analysis tasks. Metaheuristics, which denote (generally) population-based stochastic search methods, are commonly the search methods considered in such method designs. In [64], examples are given where evolutionary computation may be used for low- mid- and high-level vision tasks. Alternatively, the involvement of an efficient search method may be defined by whether it conducts tuning of a given method or is involved in the construction process of a method $[59,64,65]$. Sample supervised segment generation is defined as a tuning process in this work. Generally, such an approach may be used if there are numerous similar objects of interest in a scene, such as buildings, fields, waterbodies and vehicles.

A general overview of an example usage scenario of sample supervised segment generation (generic variant as first seen in [59]) is given along with details of the method, explained in the context of the example. Figure 1.2 illustrates an arbitrary information extraction task and depicts a subset of a VHR optical image over part of a refugee camp (the whole camp is of interest). The task may entail characterising and counting all structures to assist in logistics and planning activities for the camp. In this example, an image analysis philosophy might be followed based on the general approach depicted in Figure 1.1(a), where separate classes are depicted with separate segmentation and refinement 
processes. A sample supervised segment generation approach may assist in optimising segment quality before refinement or the initiation of other processes. Initially, a user might be interested in finding all the tents. A small selection of example objects (seven) are digitised or provided by other means, e.g. [49]. They are highlighted in Figure 1.2 by chequered yellow and black polygons and surrounded by green (and a red) rectangle(s). The tan and black chequered polygons denote additional samples, later to be used in classification.

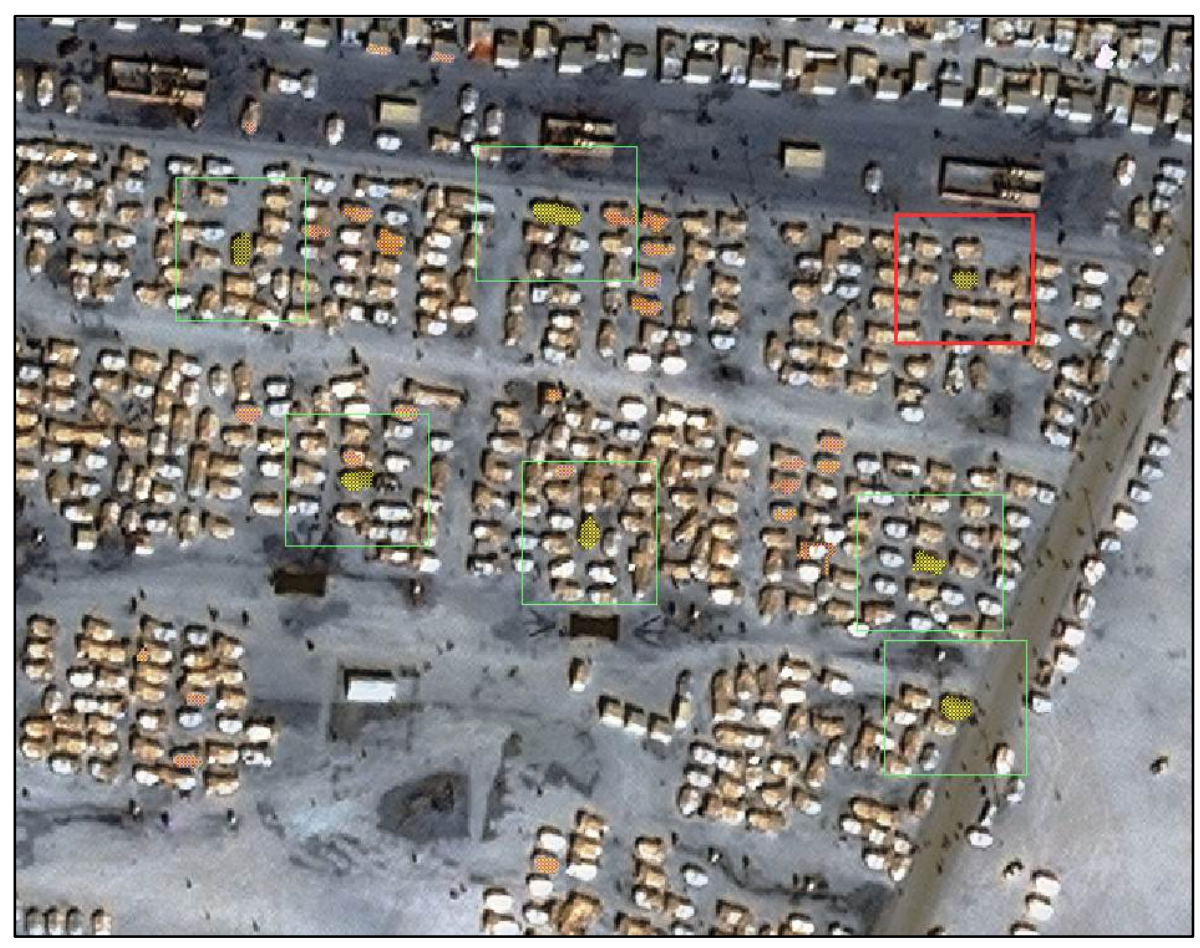

Figure 1.2. An example image analysis task where the aim could be to identify and characterise all structures. Seven tents were digitised and presented to a generic sample supervised segment generation method as reference segments.

The reference segments are extracted along with small subset imagery centred on them (rectangles). These two sets of imagery are provided as input to the general sample supervised segment generation method, shown in Figure 1.3 [63]. An iterative search process is invoked that generates parameter sets for a given segmentation algorithm, e.g. the well-known Multiresolution Segmentation [22] (MS). During each iteration of the search process, all the subset imagery is segmented (seven in this example) and the generated segments are compared with the provided reference segments. Commonly, empirical discrepancy metrics [35] are used to judge the level of similarity between generated and reference segments. The calculated quality measure, or to use metaheuristic terminology, the fitness score, is passed on to the given optimiser which uses the information to guide the search process in the next iteration (generation). 


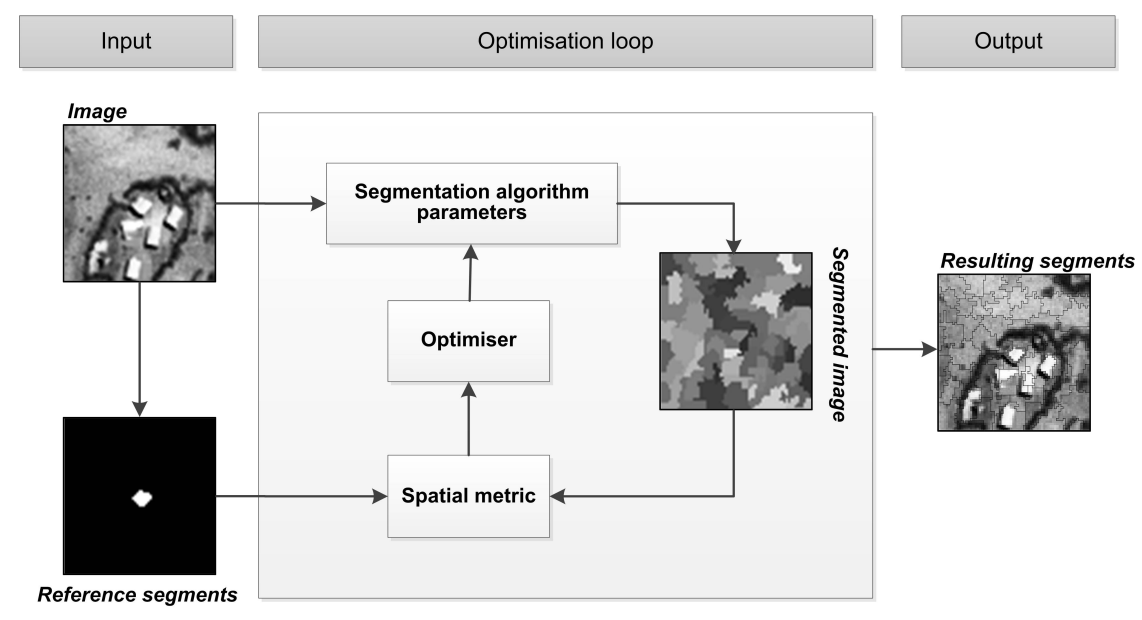

Figure 1.3. [63] The general architecture of the generic variant of sample supervised segment generation as found in $[59,62,66]$.

After a certain number of iterations have passed, or when a certain level of segment quality is reached, the method terminates and gives as output the best performing segmentation algorithm parameter set. Figure 1.4 [61] illustrates an abstract segment quality evaluation scenario where R denotes a reference segment and S (S1, S2 and S3) defines generated segments.

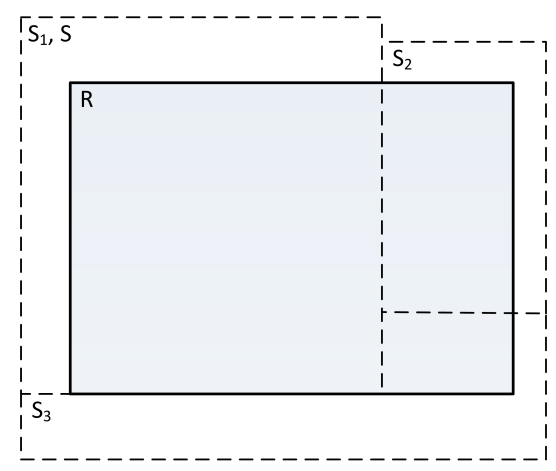

Figure 1.4. [61] An abstract segment quality evaluation scenario, where a given metric measures segment quality and provides the output to the sample supervised segment generation method.

Commonly spatial metrics are used to measure segment quality. The Reference Weighted Jaccard (RWJ) [61] measure is given as an arbitrary example (developed in this work),

$$
R W J=1-\sum_{i=1}^{n} \frac{\left|R \cap S_{i}\right|}{\left|R \cup S_{i}\right|} \times \frac{\left|R \cap S_{i}\right|}{|R|}
$$

where $\mathrm{i}$ is an iterator running over all intersecting segments $\left(\mathrm{S}_{\mathrm{i}}\right), \|$ denotes cardinality, $\mathrm{U}$ the union, and $\cap$ the difference operators.

Figure 1.5 illustrates a two-dimensional parameter slice, or search surface, generated by RWJ for such a problem using Multiresolution Segmentation (MS). Lower values indicate better segment results. Each cell depicts a given parameter set combination and thus a segmented image with said parameter set (and segment quality given by the colour/metric value). Visiting each cell in a 
multidimensional space, or brute force search, is computationally impracticable (due to segmentation and to a much lesser extent other processes) [59].

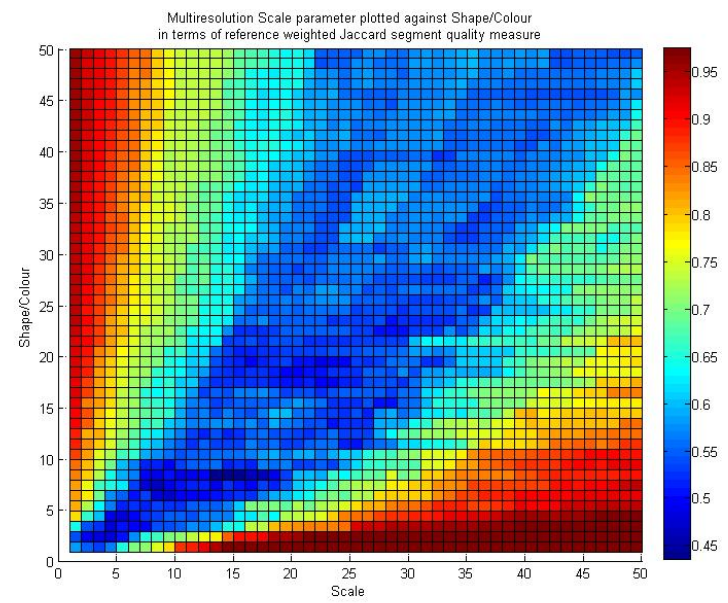

Figure 1.5. An example two-dimensional parameter slice (three dimensions in this example), or fitness landscape, generated by RWJ. Note the multimodal nature (multiple local minima).

Metaheuristics are commonly preferred to traverse the parameter set of the given segmentation algorithm [51,59-61,67-70], owing to various advantages over simpler search methods. Most importantly they are derivative-free population-based methods, less likely to fall into local optima as found in Figure 1.5. Figure 1.6 further illustrates this problem on two abstract benchmark functions, namely using a variant of Cuckoo Search [71] (programmed for this work). Note the aggregation of points in Figure 1.6(a) ("camel"), finding the true global optimal result and the failure of the method to find the optimal central optimal result in Figure 1.6(b) (“Ackley”).

(a)

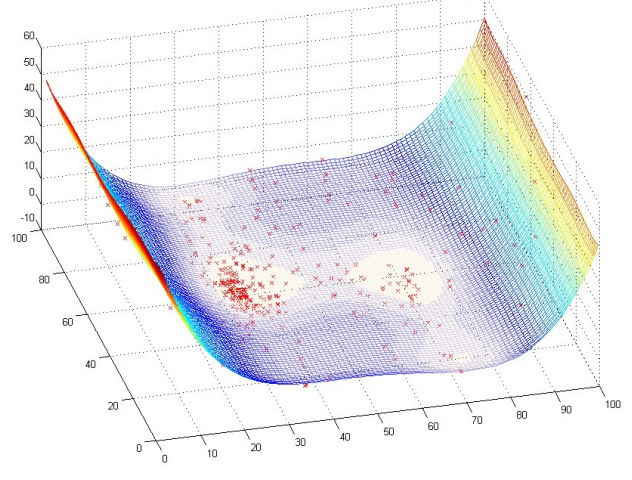

(b)

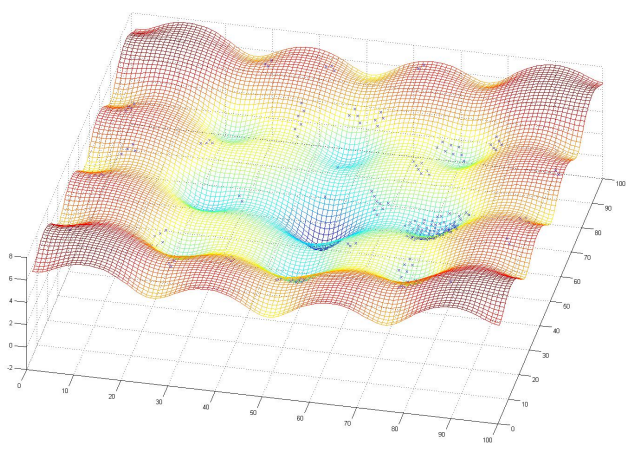

Figure 1.6. Two benchmark search problems where points depict positions visited by a given optimiser [71], illustrating an "easy" (a) fitness landscape and a "difficult" fitness landscape (b).

More detail on this general approach, including supporting concepts and a literature review, may be found in Chapter 2. See [51,70] and the Appendices for (usable) research tools based on this concept. Generally, if the method as depicted in Figure 1.3 delivers good results (as judged by metrics), such results may be used; otherwise additional processing may be needed as illustrated in Figure 1.1. 
Figure 1.7 illustrates subsequent segmentation and classification results from the problem depicted in Figure 1.2. Note that no additional processes were invoked, except automating the parameter tuning of the given segmentation algorithm (MS in this example [22]), and using a few additional examples for a manually tuned one-class Support Vector Machine (SVM) [72] (manual parameter tuning and attribute selection). More details on the software constructed for this demo (and others in the context of this work) may be found in the Appendices.

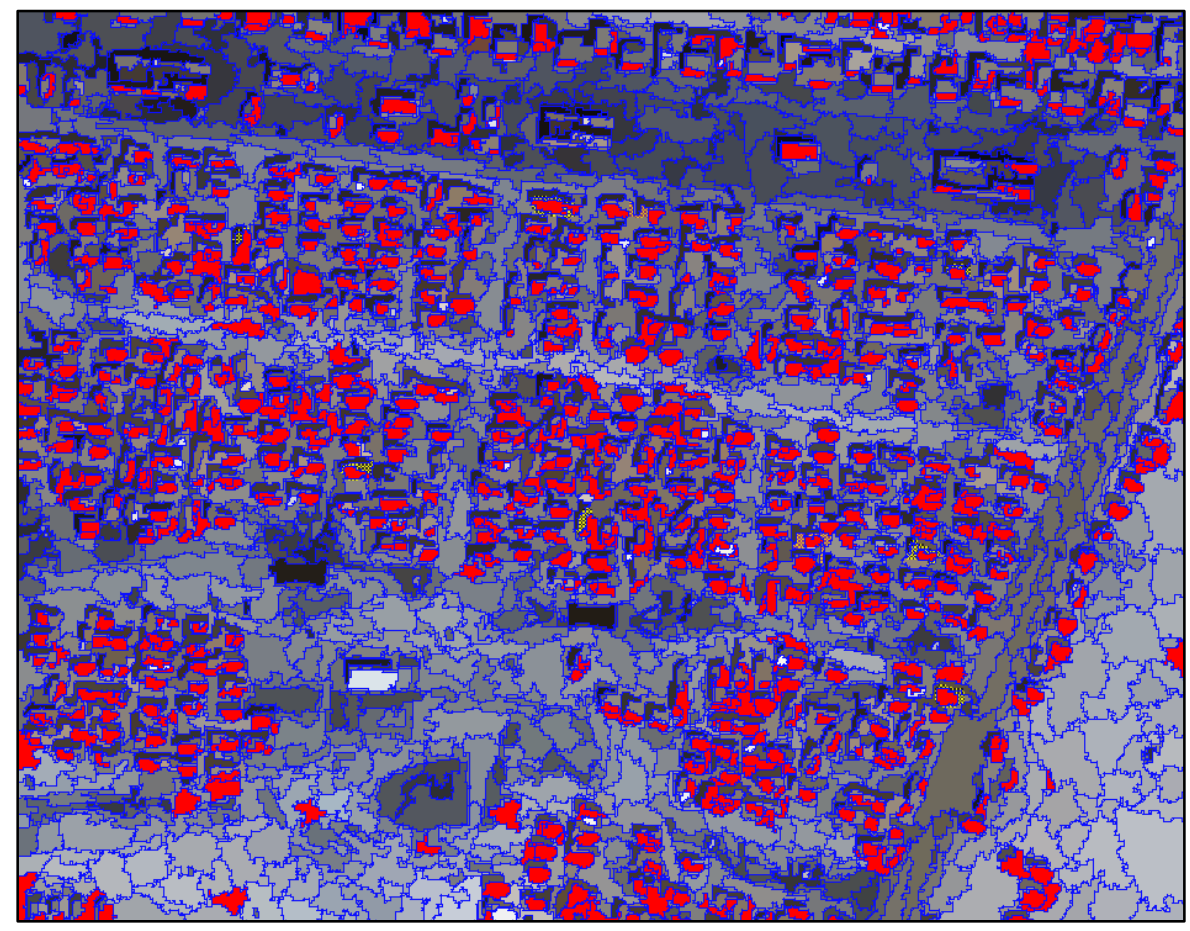

Figure 1.7. Basic classification results after running the generic variant of sample supervised segment generation and conducting basic classification (one-class SVM). Note the bad geometry of the segments related to the tents.

Figure 1.7 serves to illustrate a general problem with the sample supervised segment generation method - on all but the easiest of problems the segmentation results are insufficient to be used as is. This specific segmentation problem is truly difficult, with large variations in spectral and thematic similarity, muddled or overlapping structures and soft boundaries. Figure 1.8 illustrates an enlargement detailing the individual tents to illustrate the difficulty of the problem. Additional processes, as detailed in Figure 1.1, would be needed to derive a quality information product and a simple segmentation and classification process as depicted here would not be possible.

It should be noted that the general sample supervised segment generation method only attempts to find the optimally achievable results within the capabilities of the given segmentation algorithm. Also, this optimally achievable result might not always be found, as illustrated with the synthetic fitness landscape in Figure 1.6(b). Nonetheless, the more accurate segmentation is, in terms of over-, undersegmentation and geometry, the easier subsequent processes become. 


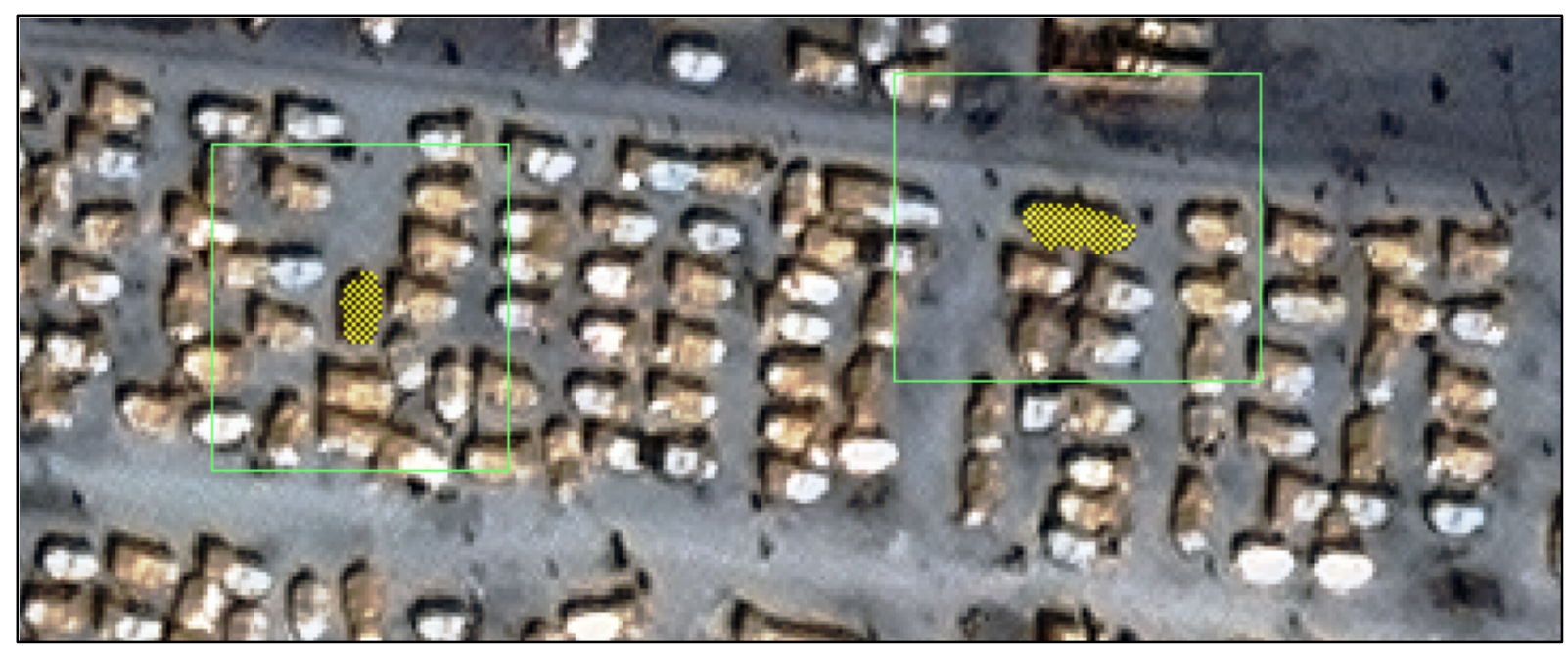

Figure 1.8. Enlargement over a part of Figure 1.2 to illustrate the difficult nature of the image analysis problem. Elements of interest (tents) are spectrally diverse, not homogeneous, not always properly separated from one another, and have soft transitions with their surroundings.

Improving on this general method has thus enjoyed research attention, e.g. $[48,50,53,67,68,73]$ (see Chapter 2 for more details). Elaborating on this general method is the focus within this dissertation. A thesis on its extension is presented next, allowing for significantly improving the quality of segments without requiring additional user interaction.

\subsection{About this Dissertation}

\subsubsection{Expanded Fitness Landscapes for Geographic Sample Supervised Segment Generation}

This dissertation explores the idea of defining expanded fitness landscapes in geographic sample supervised segment generation. Additional image processing and/or classification processes, referred to here as constituents, may be added in a sample supervised segment generation framework. Landcover element homogeneity is typically of such a nature that simple segmentation algorithms fail to achieve acceptable results, irrespective of how finely controlling parameters are tuned. This is illustrated in the above figures and is the reason for more elaborate workflows. A fitness landscape, to use optimisation terminology, defines the domain of all variables or parameters controlling a specific process. Individual points, and the accompanying parameter set, define the results of a method executed on a problem with the given parameter set, e.g. Figures 1.5 and 1.6 where the RWJ is the given quality measure. The authors of [74] present a seminal work explaining the effect of various fitness landscapes on the search performance of classical genetic algorithms.

Such expanded search landscapes may incorporate various additional constituents interacting with a given segmentation algorithm in a sample supervised segment generation method. The given segmentation algorithm may be affected by, and may affect these other constituents (e.g. see [75,76] for variable/parameter interdependency tests). This necessitates the creation of a singular fitness landscape, increasing the search problem dimensionality, difficulty and the time required to solve it. A constructed expanded search problem/landscape potentially allows for the derivation of superior results, specifically/even under conditions measuring generalizability (as done in this work). 
This idea is underscored by various general works within the optimisation and computer vision communities, where defining, analysing and solving various search problems are common [64]. Additional inspirations include the original sample supervised segmentation technique $[59,66]$ and an awareness of its limitations, various works suggesting interactions of image analysis philosophies, e.g. $[9,10,77]$, and interactions of processes within machine learning, e.g. simultaneous classifier freeparameter tuning and attribute subset selection [78].

Figure 1.9 gives an overview of the concept of expanded fitness landscapes in the context of the sample supervised segment generation method. The white shaded cells denote the general approach within sample supervised segment generation, where a segmentation algorithm functions on data and delivers an output (single-scale, multi-scale or class independent $[14,48]$ ). The shaded cells denote possible extensions via expanded fitness landscapes.

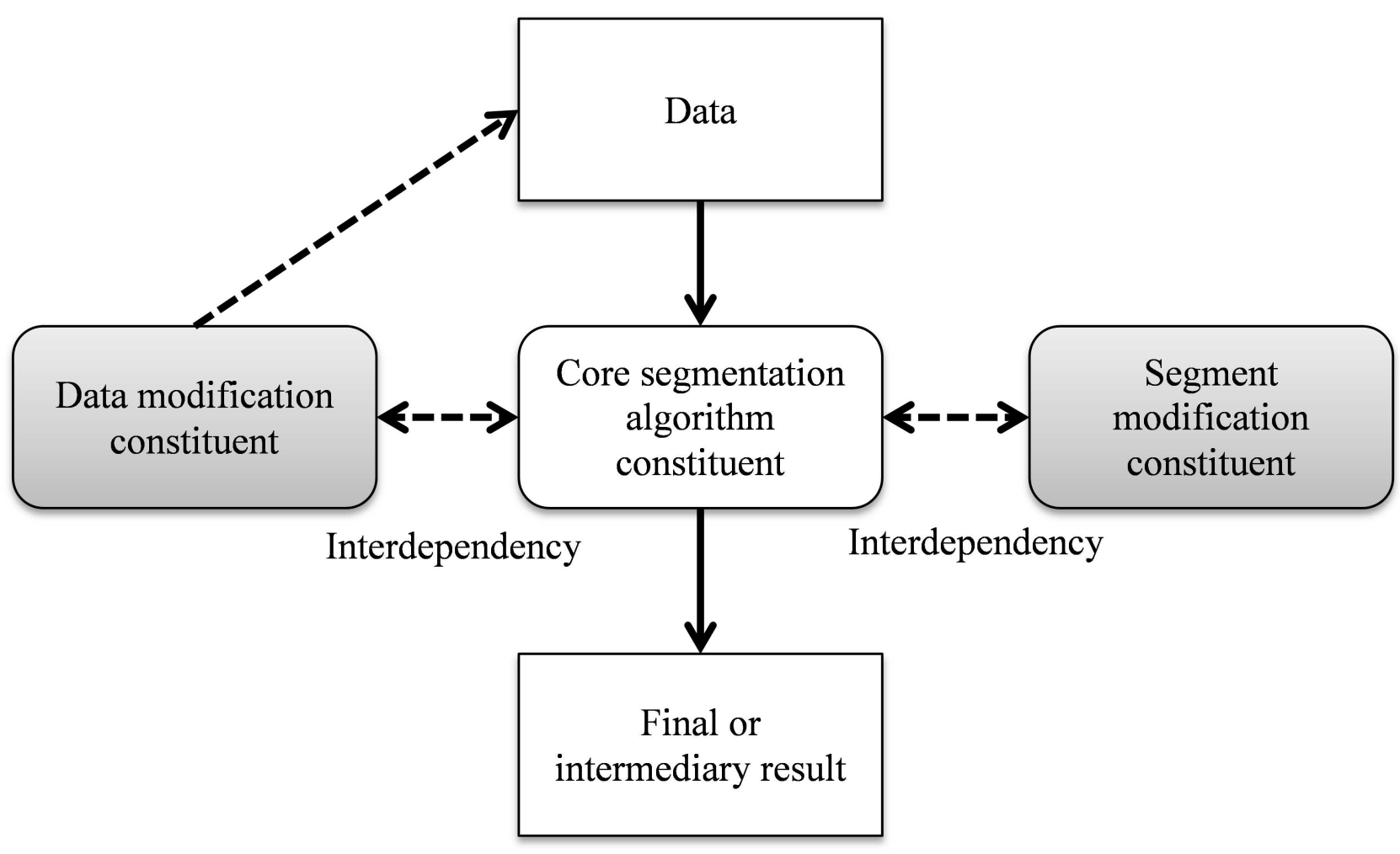

Figure 1.9. Expanded fitness landscapes in sample supervised segment generation. The shaded cells (grey) illustrate additional constituents that may be added to the general method (white cells). The three processing steps thus combine to form an expanded fitness landscape.

The data modification constituent is illustrative of processes affecting or modifying image data directly, so that the given segmentation algorithm may perform better on the given problem. In short, data mapping may force a closer thematic and spectral similarity. Generalizability may be factored in. Constituent types that may be considered here include:

- Data mapping or transformation functions (point based, neighbourhood based, colour space transforms).

- Image filters and iterative image filters (e.g. cellular automata [29]).

- Classification based data manipulation (sampling, classifier, attributes). 
The segment modification constituent suggests incorporating parameterised algorithms modifying segments via merging and splitting (or more elaborate decision processes). Such algorithms may observe segment internal or external content. Elements from these three constituents, namely the data modification constituent, core segmentation algorithm constituent, and the segment modification constituent, collectively form the expanded fitness landscape. Additionally, segmentation algorithm selection or construction may also be considered as a searchable element. The general aim in this work is thus not the presentation of new segmentation algorithms, but the exploration of a method design incorporating existing, well-studied, remote sensing image segmentation methods (e.g. [22,24,79]) and how general results may be further improved.

\subsubsection{Research Contribution}

Contributions in this dissertation are related to the general geographic sample supervised segment generation methodology. Specifically they are:

- Propose expanded fitness landscapes incorporating tuneable constituents interacting with a given segmentation algorithm.

- Propose, develop and evaluate a range of methods based on this general principle, including:

- a variant incorporating data mapping functions;

$\circ$ a variant conducting simultaneous attribute tuning and data mapping in the context of graph-based connected component segmentation; and

○ a variant using reference segment spectral content for classification to tailor data.

Other minor contributions include:

- Contribute to research methodology within the context of this method via methodological transfer from related disciplines.

- Present a new empirical discrepancy measure, the Reference Weighted Jaccard (RWJ) metric [61].

- A research software framework $(\mathrm{C}++)$ for exploration of such methods (see Appendices).

\subsubsection{Proposed and Evaluated Method Variants}

To substantiate the thesis, three method variants are proposed and evaluated that incorporate expanded fitness landscapes. Firstly, the concept of data modification and its interaction with segmentation is explored via data mapping functions. This idea was chiefly inspired by works within computer vision and remote sensing, focusing on the influences of colour space representations on image processing/image segmentation results, e.g. [80,81]. Figure 1.10 [61] illustrates this general approach, where a transformation function (data mapping) constituent forms part of the search problem or optimisation loop. Two well-known segmentation algorithms are tested within this approach, namely, Multiresolution Segmentation (MS) [22] and Simple Iterative Linear Clustering (SLIC) [79]. Four mapping functions are tested (combination of adapted and novel), having various properties. 


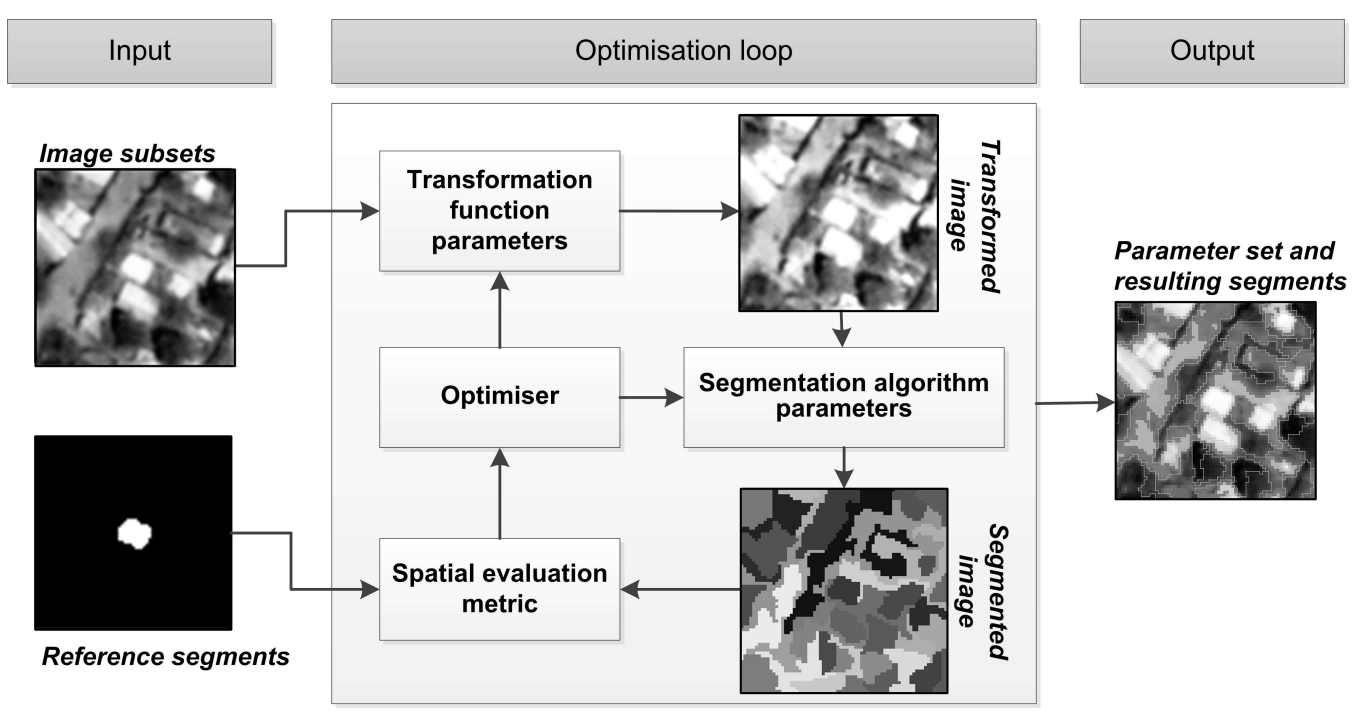

Figure 1.10. [61] Expanded fitness landscape via data mapping or transformation functions.

This general idea is subsequently extended to the domain of mathematical morphology-based image segmentation. The graph-based segmentation algorithm Constrained Connectivity (CC) [24,82-85] allows for the addition of arbitrary attributes to constrain the growth of segments, in addition to core parameters controlling the algorithm. A method variant is proposed and analysed [86] incorporating three constituents, namely those of data mapping functions, core CC parameters and a range of spectral and geometric thresholdable attributes further tailoring segments, as illustrated in Figure 1.11.

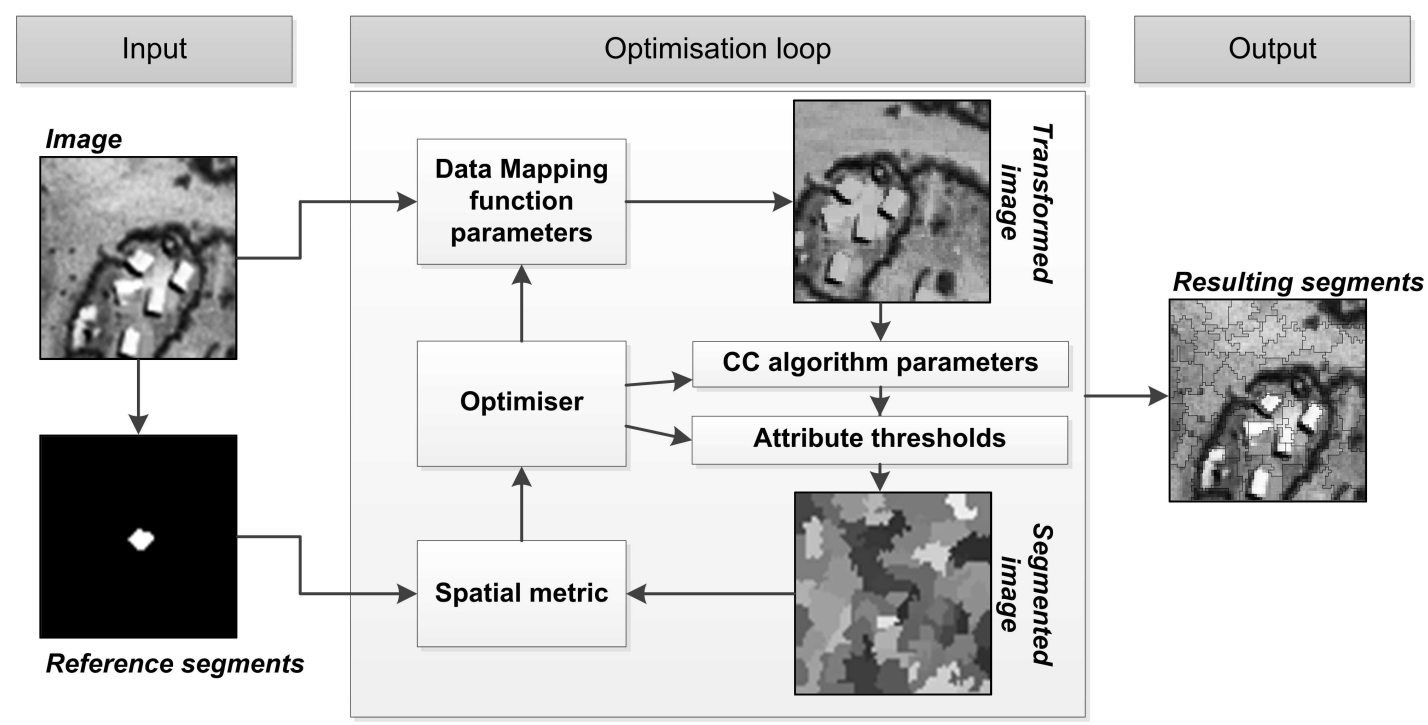

Figure 1.11. [86] Sample supervised segment generation incorporating the CC algorithm, data mapping functions and thresholdable attributes.

The general idea of utilising reference segment spectral content in some useful manner is also explored, illustrated in Figure 1.12. A method variant is proposed and analysed [88], incorporating adjustable classification components generating probability imagery, which in turn influences data mapping. The dimensionality of the fitness landscape may increase significantly in such a design. A range of method variants is investigated, based on this concept. Such a design allows for a significant 
increase in segment quality, at the expense of requiring more computing time owing to classification involved - one-class and two-class SVMs [72,87] in this instance.

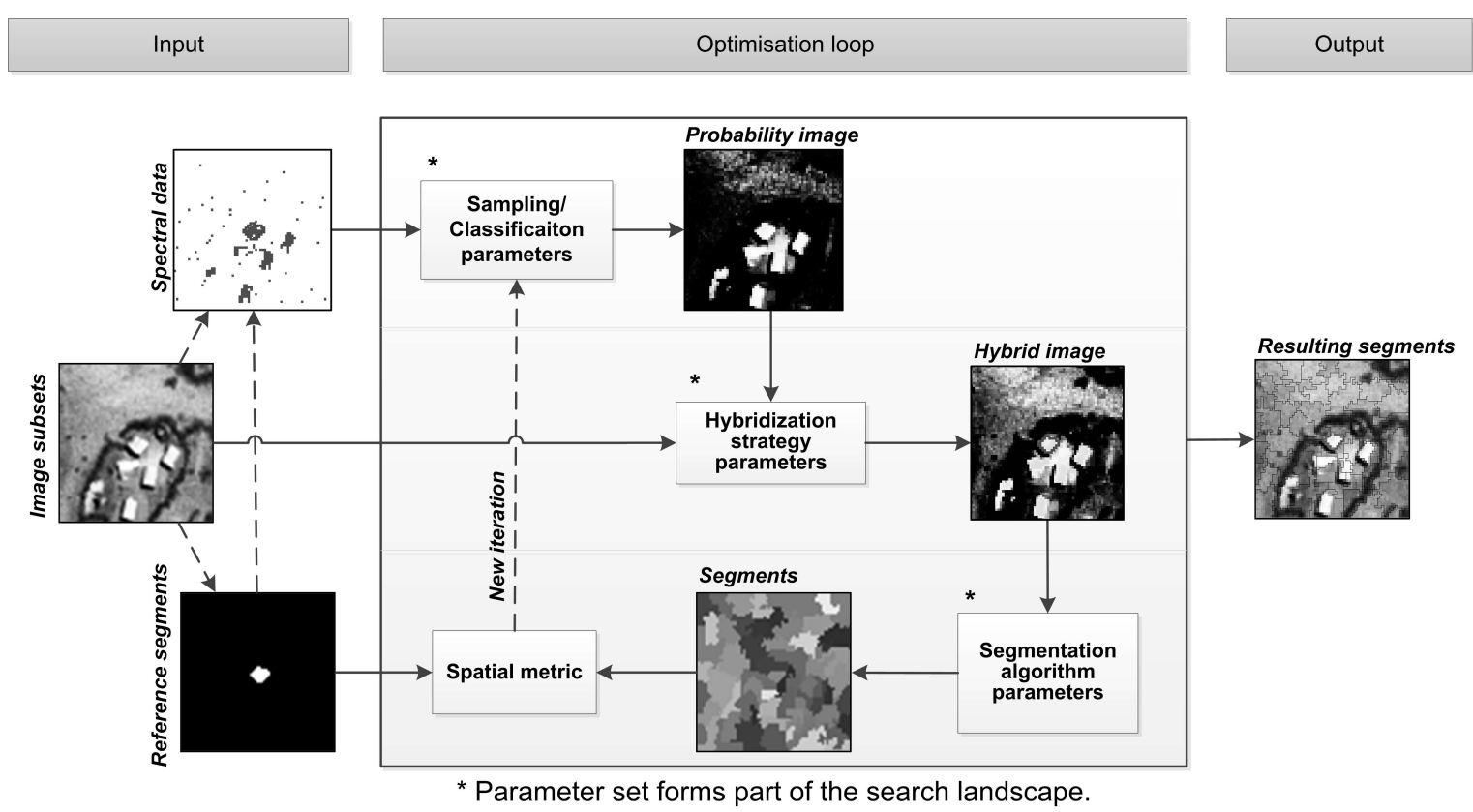

Figure 1.12. [88] A sample supervised segment generation method design incorporating classification processes.

\subsubsection{Research Design}

In this work a general quantitative comparative research design is followed, which is common within the optimisation community $[89,90]$ and also within remote sensing methodological studies. This may also be referred to as experimentalism [89] (see [91] for an interesting treatise on research/research design within evolutionary computation). Research methodology within this context may also be transferred from machine learning [92].

Proposed method variants falling within the context of expanded fitness landscapes for geographic sample supervised segment generation are analysed in two ways. Firstly they are compared with the original formulation for sample supervised segment generation in terms of classical measures such as final results, generalizability and required computing times. Numerous experimental conditions are advocated. These include using various problem instances or data sets, using four different empirical discrepancy metrics (metrics may display bias) and conducting a very large number of experimental runs (250 $000000+$ segment evaluations in Chapter 3 alone). Within each chapter the utilised datasets or problem types are described. Various statistical measures are used to report results such as the student's $t$-test, non-parametric ranking, Nemenyi post-hoc test and cross-validation $[89,90,93]$.

Secondly, the proposed method variants are analysed to render their various properties, such as the validity of the proposed methods as suitable optimisation problems. Two metaheuristics are commonly tested with all approaches, namely Differential Evolution (DE) [94,95] and Particle Swarm Optimisation (PSO) $[95,96]$. They are compared with each other and with simpler search methods (random sampling and a hill climber) to validate that these method variants are valid search problems. The relative performances of these search methods are also investigated, e.g. [97]. Parameter 
interdependencies are also examined in some method variants to affirm the interdependent nature of method constituents $[75,76]$.

\subsection{Dissertation Structure}

This dissertation has a cumulative structure. The individual chapters are published journal articles (and in one instance a peer reviewed book chapter), reproduced with publisher permission within this dissertation. In Chapter 2 (book chapter), the general concept of sample supervised segment generation is examined in greater detail, along with a review of works related to this approach. The proposed method variants as summarised above are detailed in the subsequent Chapters 3, 4 and 5. In Chapter 3, the method variant incorporating data mapping functions is presented. Chapter 4 details the method variant incorporating graph-based segmentation with additional adjustable attributes. In Chapter 5, the method variant is presented detailing the use of reference segment spectral content. A synthesis of the dissertation is given in Chapter 6, detailing research highlights, shortcomings and suggestions for future work.

The dissertation contains four sections within the Appendices. Appendix A details three conference papers presented during this research (three presentations/talks at international conferences). These papers served as pilot studies for the general methodological avenue and for the specific method variants. Experimental software was developed for each method variant. Minor details and a short user guide on the software are given in Appendix B. Appendix C contains supplementary results to explain the nature of some experimental runs (high parameter standard deviation) delivering multiple, nearly identical quality scores with vastly different parameter sets. Appendix D contains algorithm details on the DE search method and the MS and SLIC segmentation algorithms. CC segmentation is detailed sufficiently in Chapter 4.

\subsection{Publications Forming Part of this Dissertation}

Chapter 2 [63], peer reviewed book chapter - Accepted for publication:

Fourie, C.; Schoepfer, E. Sample supervised search-centric approaches in geographic object-based image analysis (geobia): Concepts, state-of-the-art and a future outlook. In Earth observation for land and emergency monitoring - innovative concepts for environmental monitoring from space, Balzter, H., Wiley-Blackwell: Hoboken, NJ, USA, 2015, in press.

Chapter 3 [61], peer reviewed journal article - Published (Science Citation Index listed): Fourie, C.; Schoepfer, E. Data transformation functions for expanded search spaces in geographic sample supervised segment generation. Remote Sensing. 2014, 5, 3791-3821.

Chapter 4 [86], peer reviewed journal article - Published (Science Citation Index listed): Fourie, C. On attribute thresholding and data mapping functions in a supervised connected component segmentation framework. Remote Sensing. 2015, 7, 7350-7377. 
Chapter 5 [88], peer reviewed journal article - Published (Science Citation Index listed):

Fourie, C.; Schoepfer, E. Classifier directed data hybridization for geographic sample supervised segment generation. Remote Sensing. 2014, 6, 11852-11882.

Appendix:

A.1 [98], peer reviewed conference paper - GEOBIA 2012 (talk):

Fourie, C.; Schoepfer, E. Combining the heuristic and spectral domains in semi-automated segment generation, Paper presented at Geographic Object-based Image Analysis (GEOBIA 2012), Brazil, 7-9 May 2012.

A.2 [99], peer reviewed conference paper - IGARSS 2013 (talk):

Fourie, C.; Schoepfer, E. Connectivity thresholds and data transformations for sample supervised segment generation, Paper presented at Geoscience and Remote Sensing Symposium (IGARSS 2013), IEEE International, Melbourne, 21-26 July.

A.3 [69], extended illustrated abstract - GEOBIA 2014 (talk):

Fourie, C.; Schoepfer, E. Classifier directed data transformations in sample supervised segment generation, Paper presented at Geographic Object-based Image Analysis (GEOBIA 2014), Thessaloniki, Greece, 21-24 May.

\subsection{References}

1. Szeliski, R. Computer vision: Algorithms and applications. Springer Science \& Business Media: 2010.

2. Sonka, M.; Hlavac, V.; Boyle, R. Image processing, analysis, and machine vision. Cengage Learning: 2014.

3. Jähne, B. Digital image processing. 6 ed.; Springer: Berlin, 2005.

4. Fei-Fei, L.; Fergus, R.; Torralba, A. Recognizing and learning object categories. Available online. http://people.csail.mit.edu/torralba/shortCourseRLOC/ (11th of April 2015).

5. Datcu, M.; Daschiel, H.; Pelizzari, A.; Quartulli, M.; Galoppo, A.; Colapicchioni, A.; Pastori, M.; Seidel, K.; Marchetti, P.G.; d'Elia, S. Information mining in remote sensing image archives: System concepts. Geoscience and Remote Sensing, IEEE Transactions on. 2003, 41, 2923-2936.

6. Zheng, S.; Yuille, A.; Tu, Z. Detecting object boundaries using low-, mid-, and high-level information. Computer Vision and Image Understanding. 2010, 114, 1055-1067.

7. Li, C.; Kowdle, A.; Saxena, A.; Chen, T. Toward holistic scene understanding: Feedback enabled cascaded classification models. Pattern Analysis and Machine Intelligence, IEEE Transactions on. 2012, 34, 1394-1408.

8. Hoiem, D.; Efros, A.A.; Hebert, M. Closing the loop in scene interpretation, Paper presented at Computer Vision and Pattern Recognition (CVPR 2008). IEEE Conference on, Anchorage, USA, 24-26 June 2008.

9. Gould, S.; Gao, T.; Koller, D. Region-based segmentation and object detection. In Proceedings of Advances in Neural Information Processing Systems, December 2009, Bengio, Y.; Schuurmans, 
D.; Lafferty, J.; Williams, C.; Culotta, A., Eds; Neural Information Processing Systems Foundation.

10. Levin, A.; Weiss, Y. Learning to combine bottom-up and top-down segmentation. International Journal of Computer Vision. 2009, 81, 105-118.

11. He, X.; Zemel, R.S.; Ray, D. Learning and incorporating top-down cues in image segmentation. In Computer vision-eccv 2006, Leonardis, A.; Bischof, H.; Pinz, A., Eds. Springer: Berlin, Germany, 2006; pp 338-351.

12. Hay, G.J.; Castilla, G.; Wulder, M.A.; Ruiz, J.R. An automated object-based approach for the multiscale image segmentation of forest scenes. International Journal of Applied Earth Observation and Geoinformation. 2005, 7, 339-359.

13. Blaschke, T.; Burnett, C.; Pekkarinen, A. Image segmentation methods for object-based analysis and classification. In Remote sensing image analysis: Including the spatial domain, de Jong, S.M.; van Der Meer, F.D., Eds. Kluwer Academic Publishers: Netherlands, 2004; pp 211-236.

14. Blaschke, T.; Hay, G.J.; Kelly, M.; Lang, S.; Hofmann, P.; Addink, E.; Feitosa, R.Q.; van der Meer, F.; van der Werff, H.; van Coillie, F., et al. Geographic object-based image analysis towards a new paradigm. ISPRS Journal of Photogrammetry and Remote Sensing. 2014, 87, 180191.

15. Blaschke, T. Object based image analysis for remote sensing. ISPRS Journal of Photogrammetry and Remote Sensing. 2010, 65, 2-16.

16. Addink, E.A.; Van Coillie, F.M.B.; De Jong, S.M. Introduction to the geobia 2010 special issue: From pixels to geographic objects in remote sensing image analysis. International Journal of Applied Earth Observation and Geoinformation. 2012, 15, 1-6.

17. Castilla, G.; Hay, G.J.; Ruiz-Gallardo, J.R. Size-constrained region merging (scrm): An automated delineation tool for assisted photointerpretation. Photogrammetric Engineering and Remote Sensing. 2008, 74, 409-419.

18. Dey, V.; Zhang, Y.; Zhong, M. A review on image segmentation techniques with remote sensing perspective. In Isprs tc vii symposium - 100 years isprs, Wagner, W.; Székely, B., Eds. IAPRS: Vienna, Austria, 2010; pp 31-42.

19. Pal, N.R.; Pal, S.K. A review on image segmentation techniques. Pattern recognition. 1993, 26, 1277-1294.

20. Cheng, H.-D.; Jiang, X.; Sun, Y.; Wang, J. Color image segmentation: Advances and prospects. Pattern recognition. 2001, 34, 2259-2281.

21. Freixenet, J.; Muñoz, X.; Raba, D.; Martí, J.; Cufí, X. Yet another survey on image segmentation: Region and boundary information integration. In Computer vision-eccv 2002, Springer: 2002; pp 408-422.

22. Baatz, M.; Schäpe, A. Multiresolution segmentation: An optimization approach for high quality multi-scale image segmentation. In Angewandte geographische informationsverarbeitung, Strobl, J.; Blaschke, T.; Griesebner, G., Eds. Wichmann Verlag: Karlsruhe, Germany, 2000; Vol. 12, pp $12-23$.

23. Wang, Z.; Jensen, J.R.; Im, J. An automatic region-based image segmentation algorithm for remote sensing applications. Environmental Modelling \& Software. 2010, 25, 1149-1165. 
24. Soille, P. Constrained connectivity for the processing of very-high-resolution satellite images. International Journal of Remote Sensing. 2010, 31, 5879-5893.

25. Dalla Mura, M.; Atli Benediktsson, J.; Waske, B.; Bruzzone, L. Extended profiles with morphological attribute filters for the analysis of hyperspectral data. International Journal of Remote Sensing. 2010, 31, 5975-5991.

26. Li, N.; Huo, H.; Fang, T. A novel texture-preceded segmentation algorithm for high-resolution imagery. Geoscience and Remote Sensing, IEEE Transactions on. 2010, 48, 2818-2828.

27. Neubert, M.; Herold, H.; Meinel, G. Evaluation of remote sensing image segmentation qualityfurther results and concepts. International Archives of Photogrammetry, Remote Sensing and Spatial Information Sciences. 2006, 36.

28. Harding, S.; Leitner, J.; Schmidhuber, J. Cartesian genetic programming for image processing. In Genetic programming theory and practice x, Riolo, R.; Vladislavleva, E.; Ritchie, M.D.; Moore, J.H., Eds. Springer: New York, United States of America, 2013; pp 31-44.

29. Rosin, P.L. Image processing using 3-state cellular automata. Computer Vision and Image Understanding. 2010, 114, 790-802.

30. Yoda, I.; Yamamoto, K.; Yamada, H. Automatic acquisition of hierarchical mathematical morphology procedures by genetic algorithms. Image and Vision Computing. 1999, 17, 749-760.

31. Frohlich, B.; Bach, E.; Walde, I.; Hese, S.; Schmullius, C.; Denzler, J. Land cover classification of satellite images using contextual information. In Proceedings of The ISPRS Workshop on 3D Virtual City Modelling, Regina, Canada, 28 May 2013, ISPRS.

32. Kersten, J.; Gähler, M.; Voigt, S. A general framework for fast and interactive classification of optical vhr satellite imagery using hierarchical and planar markov random fields. Photogrammetrie, Fernerkundung, Geoinformation. 2010, Volume 2010, 439-449.

33. Weidner, U. Contribution to the assessment of segmentation quality for remote sensing applications. International Archives of Photogrammetry, Remote Sensing and Spatial Information Sciences. 2008, 37, 479-484.

34. Zhan, Q.; Molenaar, M.; Tempfli, K.; Shi, W. Quality assessment for geo-spatial objects derived from remotely sensed data. International Journal of Remote Sensing. 2005, 26, 2953-2974.

35. Zhang, Y.J. A survey on evaluation methods for image segmentation. Pattern Recognition. 1996, 29, 1335-1346.

36. Castilla, G.; Hay, G.J. Image objects and geographic objects. In Object-based image analysis: Spatial concepts for knowledge-driven remote sensing applications, Blaschke, T.; Lang, S.; Hay, G.J., Eds. Springer: Berlin, Germany, 2008; pp 91-110.

37. Baatz, M.; Hoffman, C.; Willhauck, G. Progressing from object-based to object-oriented image analysis. In Object-based image analysis: Spatial concepts for knowledge-driven remote sensing applications, Blaschke, T.; Lang, S.; Hay, G.J., Eds. Springer: Berlin, 2008; pp 29-42.

38. Passo, D.; Bias, E.; Brites, R.; Costa, G. Comparison of the accuracy of classifications generated by interimage and by interimage integrated with data mining. South-Eastern European Journal of Earth Observation and Geomatics. 2014, 3, 93-97.

39. Kotsiantis, S.B.; Zaharakis, I.; Pintelas, P. Supervised machine learning: A review of classification techniques. Informatica. 2007, 31, 249-268. 
40. Haralick, R.M.; Shanmugam, K.; Dinstein, I.H. Textural features for image classification. Systems, Man and Cybernetics, IEEE Transactions on. 1973, 610-621.

41. Busin, L.; Vandenbroucke, N.; Macaire, L. Color spaces and image segmentation. Advances in imaging and electron physics. 2009, 151, 65-168.

42. Happ, P.; Feitosa, R.; Bentes, C.; Farias, R. A region-growing segmentation algorithm for gpus. IEEE Geoscience and Remote Sensing Letters. 2013, 10, 1612-1616.

43. Lübker, T.; Schaab, G. A work-flow design for large-area multilevel geobia: Integrating statistical measures and expert knowledge. In ISPRS Archives Vol.No. XXXVIII-4/C7, Proceedings of Geographic Object-Based Image Analysis (GEOBIA 2010), Ghent, Belgium, 29 June - 2 July 2010, Addink, E.A.; Van Coillie, F.M.B., Eds; ISPRS.

44. Hay, G.J.; Castilla, G. Geographic object-based image analysis (geobia): A new name for a new discipline. In Object-based image analysis: Spatial concepts for knowledge-driven remote sensing applications, Blaschke, T.; Lang, S.; Hay, G.J., Eds. Springer: Berlin, Germany, 2008; pp 75-90.

45. Lang, S.; Langanke, T. Object-based mapping and object-relationship modeling for land use classes and habitats. Photogrammetrie, Fernerkundung, Geoinformation. 2006, 10, 5-18.

46. Herold, H.; Meinel, G. Adaptive segmentation of heterogeneous images using metaheuristic evolutionary optimization. South-Eastern European Journal of Earth Observation and Geomatics. 2014, 3, 461-464.

47. Mylonas, S.; Stavrakoudis, D.; Theocharis, J.; Mastorocostas, P. A region-based genesis segmentation algorithm for the classification of remotely sensed images. Remote Sensing. 2015, 7, 2474-2508.

48. Musci, M.; Feitosa, R.Q.; Costa, G.A.O.P. An object-based image analysis approach based on independent segmentations, Paper presented at Joint Urban Remote Sensing Event, Sao Paulo, Brazil, 21-23 April.

49. Osman, J.; Inglada, J.; Christophe, E. Interactive object segmentation in high resolution satellite images, Paper presented at IEEE International Geoscience and Remote Sensing Symposium, Cape Town, 12-17 July 2009.

50. Ferreira, R.S.; Feitosa, R.Q.; Costa, G.A.O.P. A multiscalar, multicriteria approach for image segmentation, Paper presented at Geographic Object-Based Image Analysis (GEOBIA 2012), Rio de Janeiro, Brazil, 7-9 May 2012.

51. Achanccaray, P.; Ayma, V.; Jimenez, L.; Garcia, S.; Happ, P.; Feitosa, R.; Plaza, A. A free software tool for automatic tuning of segmentation parameters. South-Eastern European Journal of Earth Observation and Geomatics. 2014, 3, 707-712.

52. Martin, V.; Thonnat, M. A cognitive vision approach to image segmentation. Tools in Artificial Intelligence. 2008, 1, 265-294.

53. Feitosa, R.Q.; Ferreira, R.S.; Almeida, C.M.; Camargo, F.F.; Costa, G.A.O.P. Similarity metrics for genetic adaptation of segmentation parameters. In ISPRS Archives Vol.No. XXXVIII-4/C7, Proceedings of Geographic Object-Based Image Analysis (GEOBIA 2010), Ghent, Belgium, 29 June - 2 July 2010, Addink, E.; Van Coillie, F., Eds; ISPRS.

54. Chabrier, S.; Emile, B.; Rosenberger, C.; Laurent, H. Unsupervised performance evaluation of image segmentation. EURASIP Journal on Applied Signal Processing. 2006, Volume 2006, 1-12. 
55. Polak, M.; Zhang, H.; Pi, M. An evaluation metric for image segmentation of multiple objects. Image and Vision Computing. 2009, 27, 1223-1227.

56. Gueguen, L.; Soille, P. Frequent and dependent connectivities. In Mathematical morphology and its applications to image and signal processing, Springer: 2011; pp 120-131.

57. Gueguen, L.; Velasco-Forero, S.; Soille, P. Local mutual information for dissimilarity-based image segmentation. Journal of mathematical imaging and vision. 2014, 48, 625-644.

58. Drăguţ, L.; Tiede, D.; Levick, S.R. Esp: A tool to estimate scale parameter for multiresolution image segmentation of remotely sensed data. International Journal of Geographical Information Science. 2010, 24, 859-871.

59. Bhanu, B.; Lee, S.; Ming, J. Adaptive image segmentation using a genetic algorithm. Systems, Man and Cybernetics, IEEE Transactions on. 1995, 25, 1543-1567.

60. Derivaux, S.; Forestier, G.; Wemmert, C.; Lefèvre, S. Supervised image segmentation using watershed transform, fuzzy classification and evolutionary computation. Pattern Recognition Letters. 2010, 31, 2364-2374.

61. Fourie, C.; Schoepfer, E. Data transformation functions for expanded search spaces in geographic sample supervised segment generation. Remote Sensing. 2014, 5, 3791-3821.

62. Pignalberi, G.; Cucchiara, R.; Cinque, L.; Levialdi, S. Tuning range image segmentation by genetic algorithm. EURASIP Journal on Applied Signal Processing. 2003, Volume 2003, 780790.

63. Fourie, C.; Schoepfer, E. Sample supervised search-centric approaches in geographic object-based image analysis (geobia): Concepts, state-of-the-art and a future outlook. In Earth observation for land and emergency monitoring - innovative concepts for environmental monitoring from space, Balzter, H., Ed. Wiley: in preperation.

64. Cagnoni, S.; Lutton, E.; Olague, G. Genetic and evolutionary computation for image processing and analysis. Hindawi Publishing Corporation: New York, 2007; Vol. 8.

65. Bhanu, B.; Lee, S. Genetic learning for adaptive image segmentation. Springer Science \& Business Media: 1994; Vol. 287.

66. Feitosa, R.Q.; Costa, G.A.O.P.; Cazes, T.B.; Feijo, B. A genetic approach for the automatic adaptation of segmentation parameters. In ISPRS Archives - Vol.No. XXXVI-4/C42, Proceedings of Geographic Object-Based Image Analysis (GEOBIA 2006), Salzburg, 4-5 July 2006, Lang, S.; Blaschke, T.; Schoepfer, E., Eds; ISPRS.

67. Melo, L.M.; Costa, G.A.O.P.; Feitosa, R.Q.; da Cruz, A.V. Quantum-inspired evolutionary algorithm and differential evolution used in the adaptation of segmentation parameters. In ISPRS Archives Vol.No. XXXVIII-4/C1, Proceedings of Geographic Object-Based Image Analysis (GEOBIA 2008), Calgary, Canada, 29 June - 2 July, 2008, Hay, G.J.; Blaschke, T.; Marceau, D., Eds; ISPRS.

68. Happ, P.; Feitosa, R.Q.; Street, A. Assessment of optimization methods for automatic tuning of segmentation parameters, Paper presented at Geographic Object-based Image Analysis (GEOBIA 2012), Rio De Janeiro, Brazil, 7-9 May 2012.

69. Fourie, C.; Schoepfer, E. Classifier directed data transformations in sample supervised segment generation, Paper presented at Geographic Object-based Image Analysis (GEOBIA 2014), Thessaloniki, Greece, 21-24 May. 
70. Fourie, C. A one class object based system for sparse geographic feature identification. Stellenbosch University, Stellenbosch, 2011.

71. Walton, S.; Hassan, O.; Morgan, K.; Brown, M. Modified cuckoo search: A new gradient free optimisation algorithm. Chaos, Solitons \& Fractals. 2011, 44, 710-718.

72. Schölkopf, B.; Platt, J.C.; Shawe-Taylor, J.; Smola, A.J.; Williamson, R.C. Estimating the support of a high-dimensional distribution. Neural computation. 2001, 13, 1443-1471.

73. Freddrich, C.M.B.; Feitosa, R.Q. Automatic adaptation of segmentation parameters applied to non-homogeneous object detection. In ISPRS Archives Vol.No. XXXVIII-4/C1, Proceedings of Geographic Object-based Image Analysis (GEOBIA 2008), Calgary, Canada, 29 June - 2 July, 2008, Hay, G.J.; Blaschke, T.; Marceau, D., Eds; ISPRS.

74. Mitchell, M.; Forrest, S.; Holland, J.H. The royal road for genetic algorithms: Fitness landscapes and ga performance. In Proceedings of 1st European Conference on Artificial Life, 1992, Varela, J.; Bourgine, P., Eds; MIT Press.

75. Sun, L.; Yoshida, S.; Cheng, X.; Liang, Y. A cooperative particle swarm optimizer with statistical variable interdependence learning. Information Sciences. 2012, 186, 20-39.

76. Weicker, K.; Weicker, N. On the improvement of coevolutionary optimizers by learning variable interdependencies, Paper presented at Evolutionary Computation (CEC 1999), Washington DC, United States of America, 6-9 July 1999.

77. Bhanu, B.; Peng, J. Adaptive integrated image segmentation and object recognition. IEEE Transactions on Systems, Man, and Cybernetics. 2000, 30, 427-441.

78. Lin, S.-W.; Ying, K.-C.; Chen, S.-C.; Lee, Z.-J. Particle swarm optimization for parameter determination and feature selection of support vector machines. Expert Systems with Applications. 2008, 35, 1817-1824.

79. Achanta, R.; Shaji, A.; Smith, K.; Lucchi, A.; Fua, P.; Susstrunk, S. Slic superpixels compared to state-of-the-art superpixel methods. Pattern Analysis and Machine Intelligence, IEEE Transactions on. 2012, 34, 2274-2282.

80. Kwok, N.; Ha, Q.; Fang, G. Effect of color space on color image segmentation, Paper presented at Image and Signal Processing (CISP 2009), Tianjin, China, 17-19 October 2009.

81. Trias-Sanz, R.; Stamon, G.; Louchet, J. Using colour, texture, and hierarchical segmentation for high-resolution remote sensing. ISPRS Journal of Photogrammetry and Remote Sensing. 2008, 63, 156-168.

82. Ouzounis, G.K.; Soille, P. The alpha-tree algorithm. JRC Scientific and Policy Report, European Commission, Joint Research Centre. 2012.

83. Soille, P.; Grazzini, J. Advances in constrained connectivity. In Proceedings of Discrete Geometry for Computer Imagery, Lyon, France, 16-18 April, Springer.

84. Soille, P. On genuine connectivity relations based on logical predicates. In Proceedings of Image Analysis and Processing, 2007. ICIAP 2007. 14th International Conference on, IEEE.

85. Soille, P. Constrained connectivity for hierarchical image partitioning and simplification. IEEE Transactions on Pattern Analysis and Machine Intelligence. 2008, 30, 1132-1145.

86. Fourie, C. On attribute thresholding and data mapping functions in a supervised connected component segmentation framework. Remote Sensing. 2015, 7, 7350-7377.

87. Vapnik, V.N.; Vapnik, V. Statistical learning theory. Wiley New York: 1998; Vol. 2. 
88. Fourie, C.; Schoepfer, E. Classifier directed data hybridization for geographic sample supervised segment generation. Remote Sensing. 2014, 6, 11852-11882.

89. Bartz-Beielstein, T. Experimental research in evolutionary computation. Springer: Berlin, Germany, 2006.

90. Talbi, E. Metaheuristics: From design to implementation. Wiley: Hoboken, 2009.

91. Vanneschi, L.; Mussi, L.; Cagnoni, S. Hot topics in evolutionary computation. Intelligenza Artificiale. 2011, 5, 5-17.

92. Birattari, M.; Zlochin, M.; Dorigo, M. Towards a theory of practice in metaheuristics design: A machine learning perspective. RAIRO-Theoretical Informatics and Applications. 2006, 40, 353369.

93. Demšar, J. Statistical comparisons of classifiers over multiple data sets. The Journal of Machine Learning Research. 2006, 7, 1-30.

94. Price, K.V.; Storn, R.M.; Lampinen, J.A. Differential evolution: A practical approach to global optimization. Springer: Berlin, 2005.

95. Pedersen, M. Swarmops: Black-box optimization in ansi c; Hvass Lab., Southampton, UK, 2008.

96. Kennedy, J. Particle swarm optimization. In Encyclopedia of machine learning, Springer: 2010; pp 760-766.

97. Vesterstrom, J.; Thomsen, R. A comparative study of differential evolution, particle swarm optimization, and evolutionary algorithms on numerical benchmark problems. In Proceedings of Evolutionary Computation, 2004. CEC2004. Congress on, IEEE.

98. Fourie, C.; Schoepfer, E. Combining the heuristic and spectral domains in semi-automated segment generation, Paper presented at Geographic Object-based Image Analysis (GEOBIA 2012), Brazil, 7-9 May 2012.

99. Fourie, C.; Schoepfer, E. Connectivity thresholds and data transformations for sample supervised segment generation, Paper presented at Geoscience and Remote Sensing Symposium (IGARSS 2013), IEEE International, Melbourne, 21-26 July. 


\title{
CHAPTER 2: SAMPLE SUPERVISED SEARCH-CENTRIC APPROACHES IN GEOGRAPHIC OBJECT-BASED IMAGE ANALYSIS (GEOBIA): CONCEPTS, STATE-OF-THE-ART AND A FUTURE OUTLOOK
}

\author{
Fourie, C \& Schoepfer, E \\ German Remote Sensing Data Center (DFD), German Aerospace Center (DLR), \\ 82234 Oberpfaffenhofen.
}

Published in:

Earth observation for land and emergency monitoring - innovative concepts for environmental monitoring from space, 2015, Balzter, H., Ed. Wiley-Blackwell: Hoboken, NJ, USA, 2015, in press.

(C) 2015 John Wiley \& Sons, Ltd. Reprinted with publisher permission. 


\title{
Sample Supervised Search-Centric Approaches in Geographic Object- Based Image Analysis: Concepts, State-of-the-Art and a Future Outlook
} Fourie, C. and Schoepfer, E.

German Aerospace Center (DLR), German Remote Sensing Data Center (DFD), 82234,

Oberpfaffenhofen, Germany, email: fourie.christoff@gmail.com

\begin{abstract}
Sample supervised search centric image analysis has garnered research attention in various imaging disciplines concerned, to some extent, with identifying multitudes of target elements in an image. In a remote sensing image analysis context such a methodological philosophy might find value in a rapid mapping scenario or even as part of general land cover mapping approaches. A user is not involved in the decisions of how to construct or tune image processing for the given information extraction problem, but is rather tasked to give a few examples of the elements of interest, similar to the concept of supervised classification. The information extraction method is automatically constructed, with the construction process guided by various notions of quality derived from comparing the limited user provided samples with generated results. Such a design philosophy holds substantial value for rapid or emergency response mapping endeavours such as the Copernicus Emergency Management Services (EMS). Current land-cover mapping methodologies and technologies are not tailored to the needs of rapid mapping. The concept of sample supervised optimisation based image analysis is examined, in which an efficient search algorithm is used, in various ways, to construct or tune a method. The application of such a general search centric information extraction philosophy within the discipline of Geographic Object-based Image Analysis (GEOBIA) is reviewed, with a few discussions given on limitations of such a general approach. GEOBIA, as a sub discipline of both geographic information science and remote sensing, denotes various methodological approaches to image analysis centered on the concept of semantic segmentation. Finally, considering developments in sample supervised image analysis from outside of the domain of remote sensing, a synthesis is presented on potential avenues for research on sample supervised search centric methods within GEOBIA.
\end{abstract}

\subsection{Search Centric Sample Supervised Image Analysis}

\subsubsection{Image Analysis as a Design Problem}

Image analysis signifies the process of extracting required or useful information from a digital image, typically using various forms of image processing and classification. Research on image analysis spans several diverse and well established disciplines; some focused on specific problem domains and applied research [1] while others are concerned with more primary, problem insensitive inquiries [2, 3]. Some example domains where image analysis research forms a prominent part of activities include digital image processing, satellite remote sensing, biomedical image analysis, astronomy, mathematical imaging, image information mining and computer vision. The intrinsic 
characteristics of the images in these domains and thus the developed approaches to image analysis are as diverse as the fields that are concerned with it.

Image analysis processes can range from simple expert system classifier based approaches observing the spectral values of basic elements in an image (e.g. pixels), to complex processes considering spatial [4] and hierarchical context relationships, using numerous feature descriptors (attributes of image elements) [5], combining various primary analysis paradigms such as semantic segmentation, template matching, discriminative models and parts based models [6] and also involving various approaches taken from machine learning, e.g. as applied in context based image retrieval [7] and optimization [8].

Remote sensing image analysis poses as an interesting domain where the nature of the used analysis techniques evolved due to the progression of the fidelity of the spatial resolution of the captured imagery [9]. Classically image analysis within this domain was conducted with simple supervised, unsupervised or expert system's classification approaches functioning on the spectral values of pixels in multi-band images. Typically on modern very high resolution (VHR) optical imagery, approaches attempting to conduct semantic segmentation along with using context information are more common [10]. Irrespective of the domain in which the image analysis is performed, the method, consisting of image processing and/or classification techniques, used to conduct the information extraction needs to be designed.

\subsubsection{Metaheuristics as Tools for Design}

Metaheuristics, which signify a family of efficient problem independent stochastic search algorithms are well suited and extensively studied in various fields as efficient tools to aid in design processes $[11,12]$ despite the non-deterministic nature of the methods and lack of theoretical models to many algorithms [13]. Within the fields concerned with image analysis and especially computer vision, metaheuristics have been studied and applied extensively in extremely varied ways to design solutions $[14,15]$.

Figure 2.1 illustrates a simple general architecture common to most population based metaheuristics. An initial population is defined, typically randomly initiated, consisting of a group of candidate solution vectors or agents to a design problem. An individual agent within the population contains all the building blocks for a method, encoded as real, integer, discrete or binary values, depending on the specific details of the given metaheuristic. These values can control, amongst others, parameters for pre-exisiting image processing components. Although problem specific, values within agents are typically interdependent.

A metaheuristic search method proceeds iteratively. These iterations are referred to as generations if the metaheuristic is nature inspired (e.g. as with evolutionary inspired algorithms such as genetic algorithms and differential evolution). Each agent of the population undergoes a fitness evaluation during iterations of the algorithm, which denotes the process of calculating how well the agent performs on the given problem. This evaluation process is defined by a fitness function, also called an objective function. After the fitness evaluations of all agents in the population the method termination condition is queried. If any agent in the population has a fitness score (quality of the solution) greater than a specified threshold, if a certain number of method iterations have passed or if very little change is observed among subsequent method iterations, the method terminates. The agent possessing the best 
solution vector represents the result of the search process. If the method does not terminate, a new population is created. The agents of the new population may be defined via combining algorithms utilising different agents in the population, some randomization and knowledge of how an agent performed compared to previous iterations.

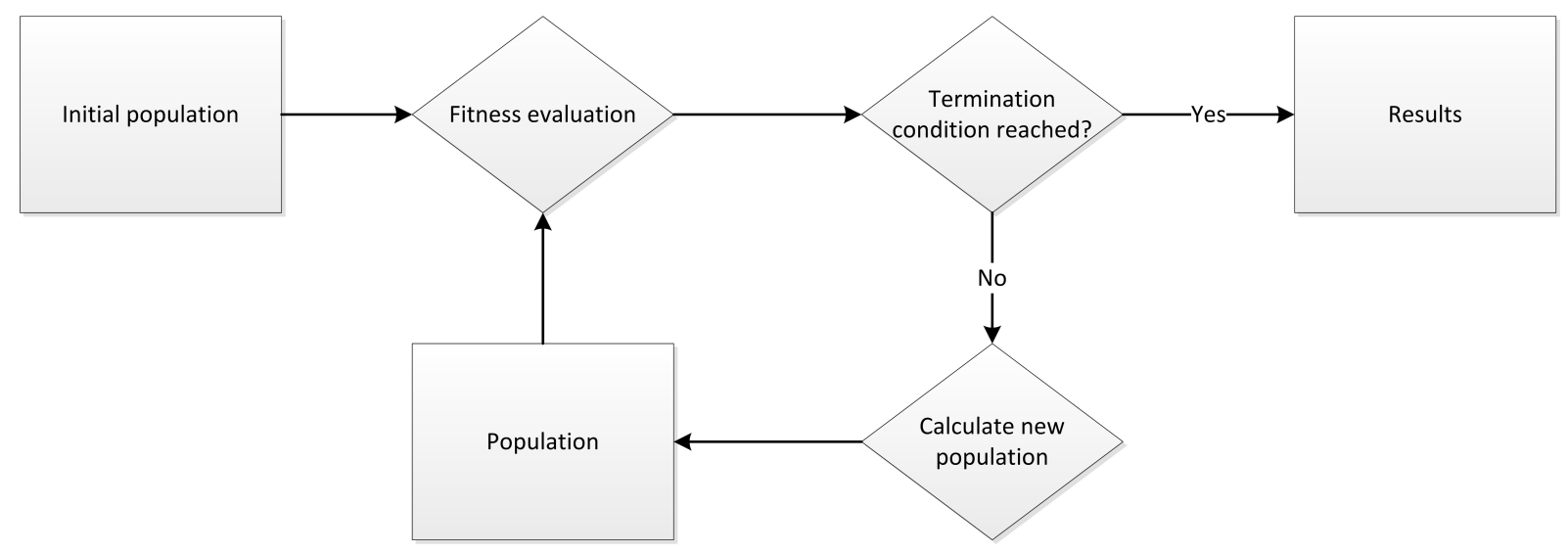

Figure 2.1. Generalised architecture of simple population based metaheuristics.

The domain or space explored by metaheuristics is often referred to as the search landscape. Figure 2.2 illustrates a two dimensional search landscape, defined by a common benchmark function (Ackley's function). The red dots represent positions visited by a metaheuristic during the search process, in this instance an adaptation of modified cuckoo search [16]. Lower z-axis values imply better solutions.

Figure 2.2 also illustrates the concept of local optima, where the central global optimal position in terms of solution quality is surrounded by eight solution positions of lesser quality, although performing better than their immediate surroundings. Note the agglomeration of agents around the central global optimal position. The use of metaheuristics are routinely justified on such problems, where simpler single agent strategies, either using search landscape gradient information or not, struggles to escape from such local optima [17]. Metaheuristics use the concepts of exploitation and exploration to efficiently traverse search landscapes in search of the global optimum: agents typically use information from their own best performances in addition to information from the performances of other good performing agents to modify their own values [11, 17]. From a design perspective, the ways in which one could encode solution vectors as control processes to generate search landscapes such as in Figure 2.2 are boundless. 


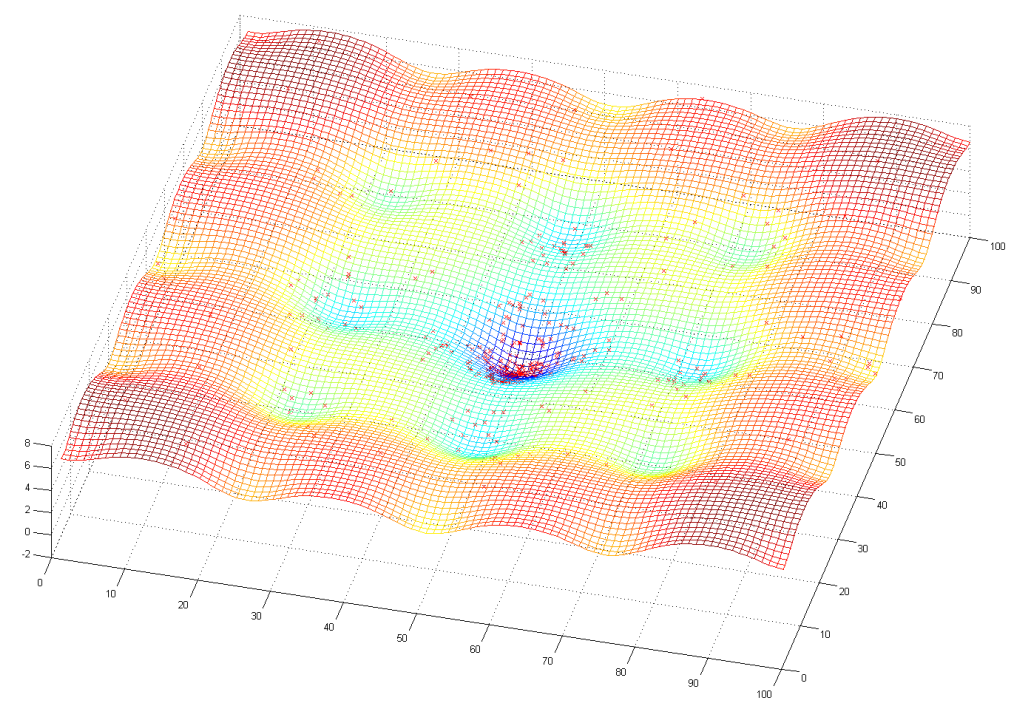

Figure 2.2. An arbitrary search landscape, with positions visited by a metaheuristic during the search process denoted with red dots.

Specific solution vector encodings related to image processing and image analysis could be extremely complex and require extensive fitness evaluations and computing time. Metaheuristics may assist in addressing such problems by attempting to solve them faster and obtaining more robust results compared to classical search methods [11]. Figure 2.3 illustrates two fitness traces of two search algorithms. A fitness trace signifies the quality of the best agent/solution vector as the iterative search process proceeds (e.g. the solution vector quality of the best performing red dots in Figure 2.2 as a function of iterations). The fitness traces in Figure 2.3 are generated by the Differential Evolution (DE) metaheuristic and Random Search (RND) for an arbitrary seven parameter design problem in image analysis. For this design problem, the use of the DE metaheuristic is justified over such a simpler search strategy. A lower solution quality signifies better results.

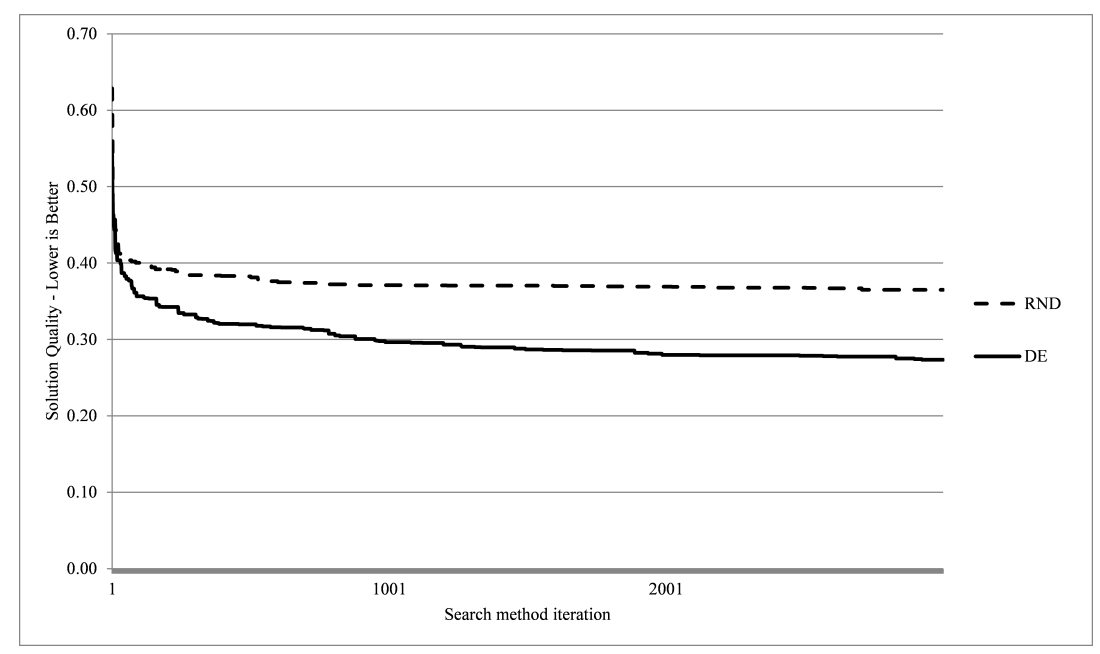

Figure 2.3. Fitness traces generated by two different search methods for an arbitrary design problem in an image analysis context. 
In a search process, each fitness evaluation is assigned with a unit of computational cost. Commonly for many problems, including problems in the image analysis domains, such potentially expensive fitness evaluation costs are the main drivers for attempting to search as efficiently as possible. Metaheuristics typically only explore a fraction of the search landscape compared to brute force search or simpler search strategies [18] to obtain optimal or near optimal solutions. In many practical instances this allows for design to be performed with metaheuristics. The time taken to search for a solution is also influenced by the problem difficulty or the nature of the search landscape [13] as defined by the fitness function. Metaheuristics are typically evaluated with black box optimsation benchmarks [19] using experimental research methodologies [20].

\subsubsection{Granularity and Fitness Functions in Sample Supervised Search Centric Image Analysis Method Design}

It can be argued, and has been alluded to $[12,14,18]$, that the way in which a metaheuristic is used in image analysis method design may be described by the granularity of method construction. Granularity defines the fineness or coarseness of the basic building blocks of the method design process. At a coarse level, agent values could simply define free parameters of an image processing method that needs to be tuned, for example the parameters of a segmentation algorithm [18, 21-23] or the parameters (e.g. a threshold value to identify water in a satellite image) of an expert system's classification approach. At a finer granularity, the building blocks could be more basic units or definition processes, such as defining structuring elements in classical mathematical morphology image processing [24], defining cellular automata rules [25], finding a sequence of good image processing using genetic programming [8] or even for defining the nature of attributes used in classification processes [26].

More complex information extraction frameworks are also possible, for example approaches that interleave object recognition and machine learning in the search process [27, 28] or even approaches that search for coarse grained methods within the search process [29]. It can be debated that the finer the granularity of the building blocks, the higher the dimensionality of the search landscape, and the more difficult the search problem becomes. Alternatively stated, the finer the granularity, the more search time would be needed. On the other hand, finer grained processes might execute faster (per fitness evaluation) than coarse grained processes and would probably be more flexible to various problem instances.

Keeping in mind the granularity of method building blocks that defines the search landscape complexity, another major consideration to using metaheuristics in design is how to define the fitness function. In image analysis method design, solution quality can be defined by various quality measures describing the quality of the final information product or only intermediary steps in the image analysis process. The output could thus be new intermediary data (data in, data out) or information.

Two common fitness function groupings are identified, measures observing low- and mid-level processes, specifically segmentation results, and classification accuracy measures. Empirical goodness methods [30, 31] define unsupervised notions of image simplification or segmentation quality, typically by evaluating statistical measures from generated image areas or segments. Empirical discrepancy measures [30] are supervised quality measures, where generated results are measured against provided ground truth, reference or gold standard examples. With classification, user's and 
producer's accuracy, kappa, geometric means, f-score and various other quality criteria could measure the generated results against the provided reference results.

Thus, search centric sample supervised image analysis and search centric sample supervised segment generation denotes method design processes where a sample or selection of reference elements is provided. The result of a fitness evaluation is how well certain generated elements match with provided reference elements. An interesting, but potentially computationally prohibitively expensive alternative to searching for a solution is to calculate all possible solutions in an ordered manner (e.g. tree hierarchies as used with interactive image information mining) and extract the set that matches most closely with the provided reference elements [32].

\subsection{Approaches in Geographic Object-Based Image Analysis}

GEOBIA defines a general image analysis philosophy centered on the concept of semantic segmentation [9, 33, 34]. As an important objective GEOBIA addresses the development of methods and accompanying theory to replicate a human's ability to interpret remote sensing imagery in automated and semi-automated ways [35]. The proliferation of VHR data, increased computing power and closer integration between Geographic Information Systems (GIS) and remote sensing processes are often cited as driving forces to the development of GEOBIA [9, 33]. When concerned with VHR data, basic thematic units may be much larger than individual pixel sizes. Attempting to classify such imagery with pixel-based approaches often leads to the so-called speckle or salt and pepper effect in classification results [33].

Central to GEOBIA approaches is the generation of image objects [36], typically using various image segmentation algorithms. Other common constituents in GEOBIA include classification, attribution and information presentation considerations [37]. A distinction is made between image objects, which are arbitrary image segments and geographic or geo-objects, which denote thematic elements of interest $[35,36]$. Progressing from image objects to thematic objects typically proceeds in two ways, either via attempting to segment elements of interest correctly from the start, or by using various post segmentation processes to progress to semantic objects. In remote sensing image analysis problems, elements of interest may be numerous, and numerous types of elements might also be of interest.

In some problem instances, especially if the spatial and spectral characteristics of elements of interest are similar, a thematic segmentation or geographic object generation process may be attempted with a segmentation algorithm by tailoring its parameters to the problem. Figure 2.4 illustrates such a scenario and depicts white tents of similar geometric and spectral characteristics, segmented with an arbitrary segmentation algorithm. In other scenarios, elements of interest that are thematically identical might be too different to allow for simple segmentation.

For more complex image analysis problems where semantic segmentation cannot be achieved easily, various approaches within GEOBIA have been proposed. A multi-scale image analysis philosophy might be advocated, where a scale-scape constraint segmentation algorithm is run multiple times to create a hierarchy of image objects $[32,38]$. Information through the hierarchy might be used to identify proper thematic objects (classification) in addition to using context and attribute information within a specific layer, e.g. [39]. 


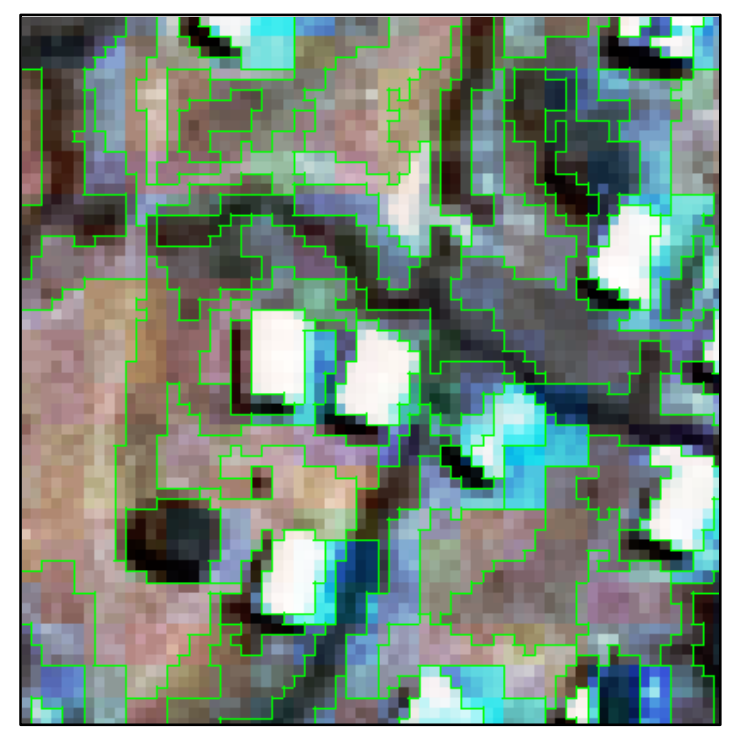

Figure 2.4. A simple segmentation problem where spectrally and geometrically similar elements of interest are segmented with a single segment layer.

A prominent strategy for addressing both simple and complex problems within GEOBIA is an expert system's or rule set/rule based approach [9, 10, 33]. The various image processing and classification, and sequences of processes needed to identify elements are defined by an expert user. The set of methods or procedures designed are encapsulated in a rule set. Such a rule set is typically created on a specific input image, but it may be used to extract information on other imagery.

Common procedures in rule set development include performing general image processing, defining image segmentation operations with various parameters to tune, merging and splitting segments, assigning or defining attributes attached to segments and classifying segments using expert knowledge or supervised/unsupervised classifiers. Using segment relations as information in hierarchical segmentation layers and in segments of a single layer is also common. Rule sets may contain additional processes and may also display complexity in orderings or sequences of processes [10]. Rule set development is commonly facilitated with commercial or free image analysis software $[33,40,41]$.

Although this general strategy in GEOBIA is highly advocated, especially in problem instances with highly complex image details [9], two general concerns could be raised. Firstly, the transferability (generalizability) of rule sets to other geographic areas or by using data captured by different sensors may be a concern and are not routinely, explicitly incorporated into method designs. Secondly the process of designing a rule set is time consuming and highly dependent on the skill of the designer. One of the primary problems identified in this regard is selecting and tuning a segmentation algorithm for a specific scenario. Some approaches address this problem by using various empirical goodness measures to automatically define good segment layers [38, 42]. Another general approach utilizes search methods to find good segment layers, which is presented next. 


\subsection{Search Centric Sample Supervision within GEOBIA}

\subsubsection{Automatic Segmentation Algorithm Parameter Tuning}

Within GEOBIA a general search centric automatic segmentation algorithm parameter tuning approach has been proposed due to the need for more streamlined processes [21, 23, 43, 44], especially when considering rule set approaches. Due to the computationally expensive nature of image segmentation a thorough search of the search space is commonly not feasible, requiring the use of efficient search methods. This general approach draws inspiration from earlier works from outside the domain of remote sensing [18, 22] and is also actively pursued in other imaging disciplines, e.g. [28]. Figure 2.5 illustrates a generalised architecture of such a method. The method attempts to find good segments based on a limited number of user provided reference segments or objects. A user provides reference segments either via digitizing or with other available input methods [45]. Other uses of metaheuristics have also been demonstrated in this domain, e.g. for feature selection [46].

During the optimization loop iterations, the search algorithm gives as output a parameter set for the given segmentation algorithm. Next the image, or subsets of the image for efficiency, is segmented with the tuned segmentation algorithm. The segmented image is compared with the provided reference segments to generate a quantitative measure of quality. This constitutes the fitness evaluation in the search process. The result of the fitness evaluation is passed on to the optimizer, which uses the information to direct the search process in subsequent iterations of the search method. Various stopping criteria could be specified for this general method, as is common for metaheuristic approaches.

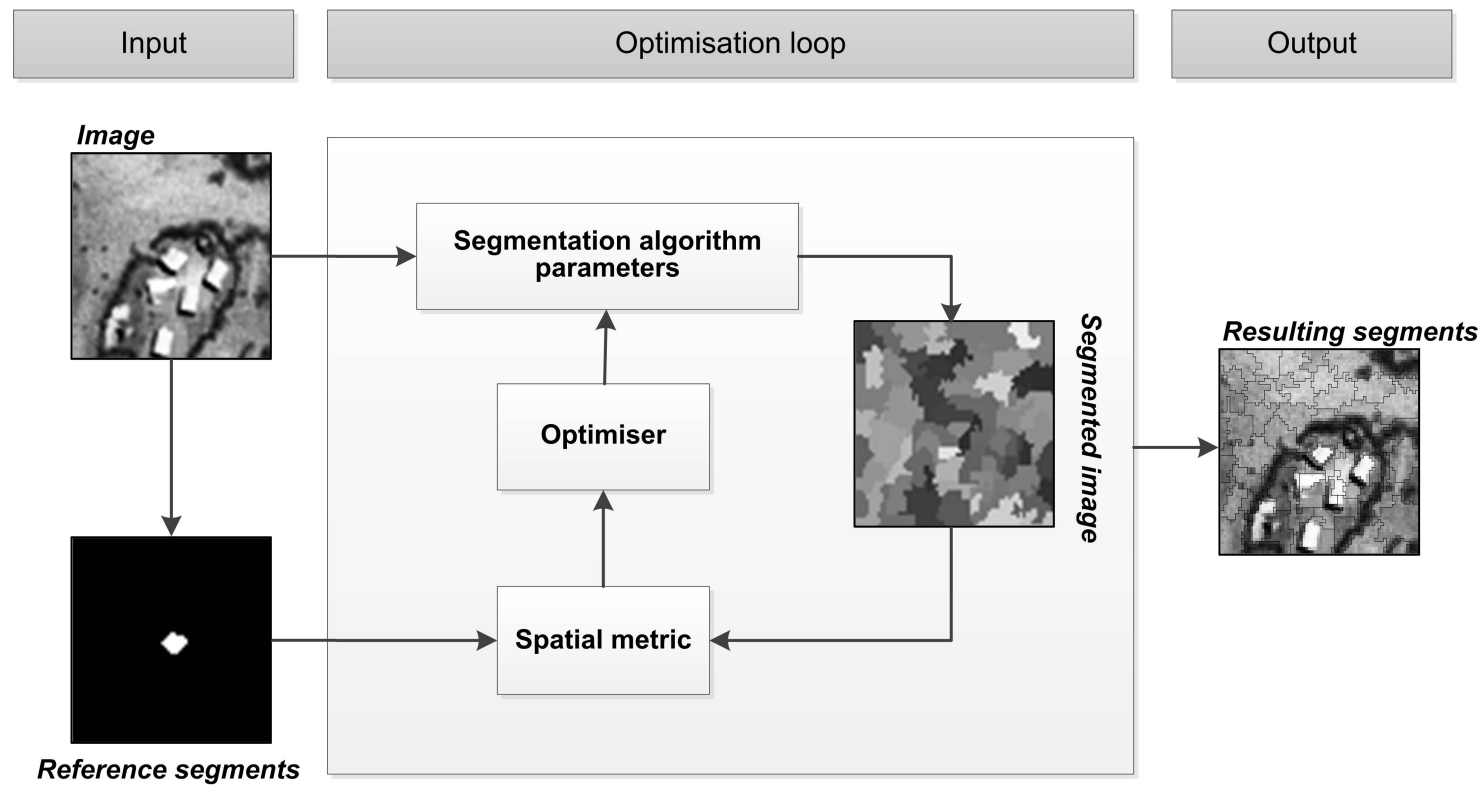

Figure 2.5. Generalised architecture of a search centric sample supervised segment generation approach.

Figure 2.6 illustrates an example scenario of how such a general approach may be used. A user might select or digitize elements of interest (Figure 2.6a), in this example tents in a refugee camp. The user may view the results of the automatic segmentation algorithm parameter tuning process (Figure 
2.6b), followed by a classification procedure to identify similar elements, for example by using a novelty detector and the reference objects as training data (Figure 2.6c).

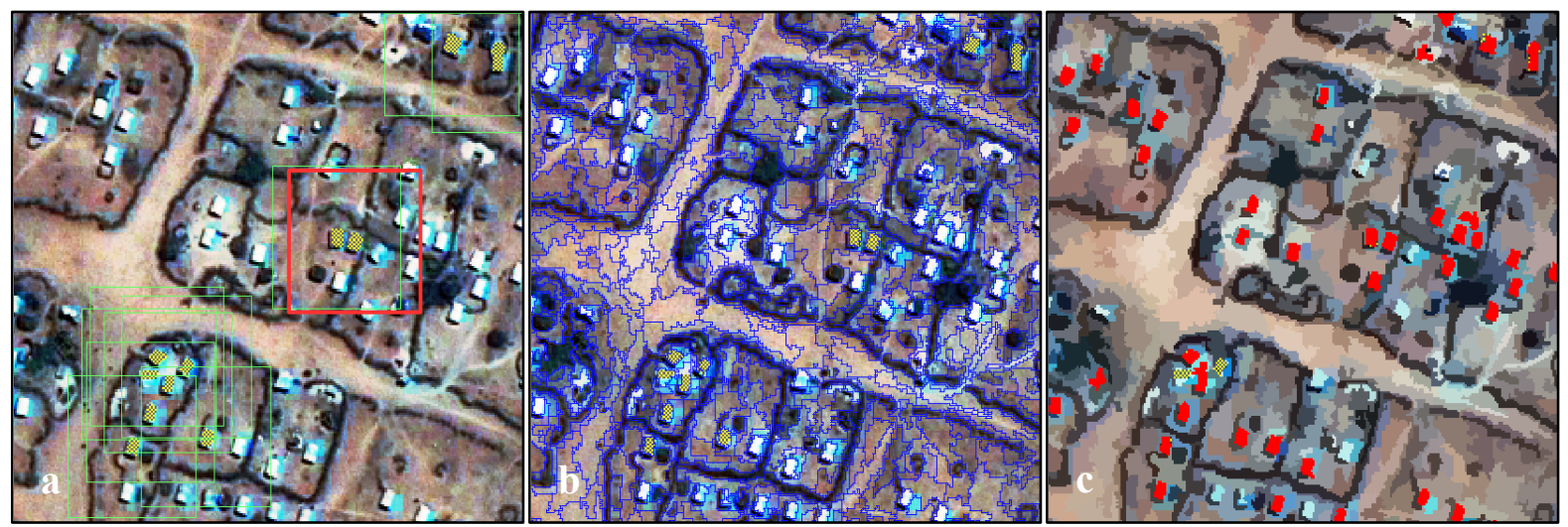

Figure 2.6. An example mapping scenario where such an automatic segmentation algorithm parameter tuning process may form part of the image analysis workflow.

Research surrounding this general approach strives to show its practical feasibility and performance in a remote sensing and GEOBIA context. Research focussed tools with graphical user interfaces have been developed [47-49] for such an approach due to its interactive and visual nature. The freeware Segmentation Parameter Tuning (SPT) tool [49] allows a user to automatically tune a range of segmentation algorithms under different metric and search method conditions, along with the ability to export results for further use. Geographic Object Novelty Detector (Geo-ND) [47] is a simple proofof-concept tool illustrating a complete workflow incorporating target detection using automatic segmentation algorithm parameter tuning in addition to classification processes.

\subsubsection{Measuring Segment Quality}

An important aspect of this general approach described is how to measure the amount of similarity between the generated segments and the reference segments. Quality is typically measured with empirical discrepancy methods [30] with various imaging disciplines making use of such techniques. In GEOBIA and more generally in remote sensing numerous quality measures have also been proposed, although not always used as fitness functions in search processes [21, 50-58]. Measures typically observe notions of area overlap (e.g. Figure 2.7) where a reference segment (R) is matched for spatial coherence with a generated segment (S). Notions of over-segmentation, undersegmentation, geometry, edge-offsets and spectral properties may also be encoded into such quality metrics.

Figure 2.8 illustrates three image subsets with an example reference segmented delineated by a bold red polyline. The objective could be to accurately segment buildings. During the search iterations of the method depicted in Figure 2.5, such evaluations are performed. Quality scores of the Reference Weighted Jaccard (RWJ) measure is also shown [48], with lower values signifying better segmentation results, with a range of [0,1]. Figure 2.8(a) illustrates a segmentation algorithm parameter set and accompanying image segments (green lines) resulting in an over-segmentation. Figure 2.8(b) 
represents a better or adequate segmentation, while Figure 2.8(c) represents an under-segmentation scenario.

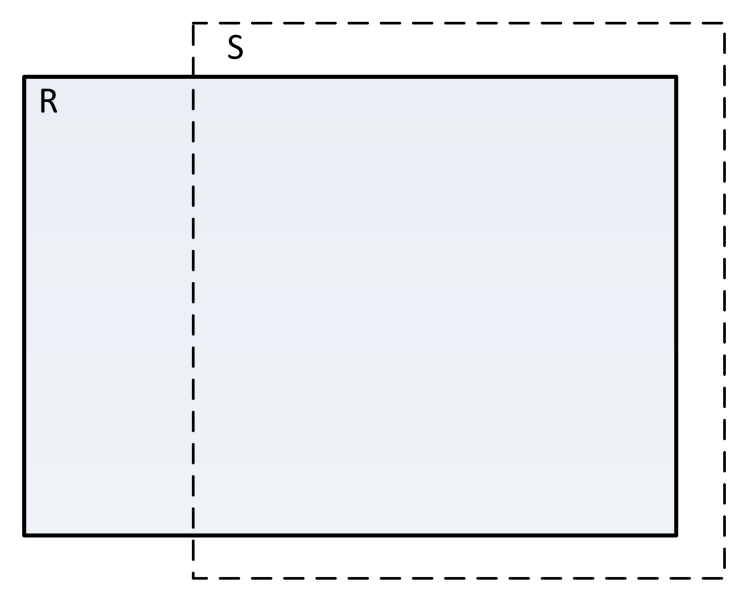

Figure 2.7. A representation of area overlap. Empirical discrepancy measures or spatial metrics could measure the amount of spatial overlap between the reference $(R)$ and a generated (S) segment.
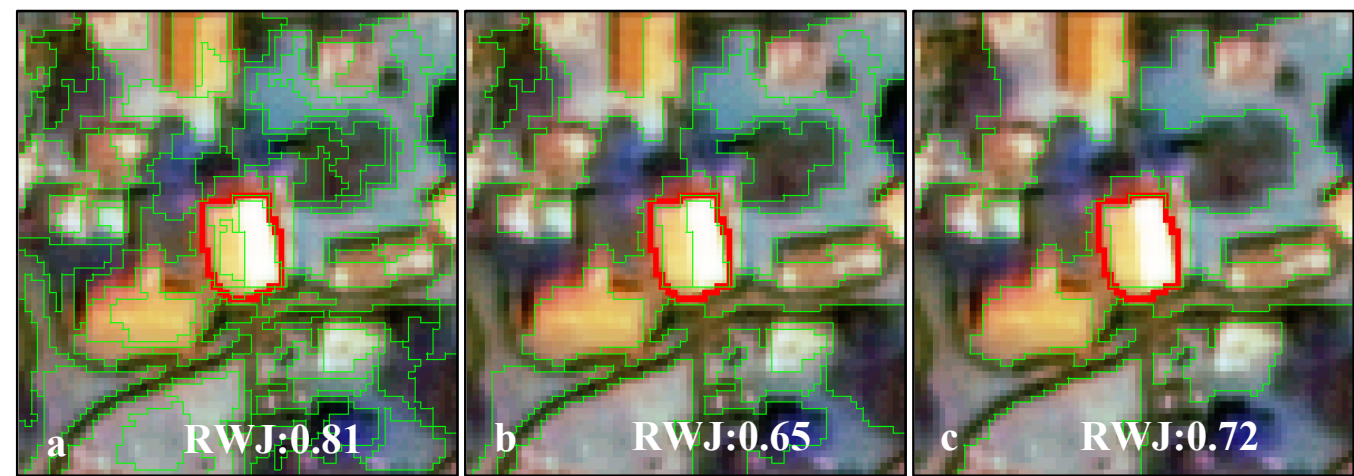

Figure 2.8. Three segmentation evaluation examples using the RWJ metric evaluating the mismatch between reference (red line) and generated (green lines) segments.

\subsubsection{Advances in Search Centric Sample Supervised Segment Generation}

Various aspects of such a sample supervised search centric method have been subjected to further investigations within GEOBIA and remote sensing. The behaviour of using spatial metrics have been shown to be highly correlated [55] on a metric test bed consisting of general area-overlap metric variants (e.g. Figure 2.7). Multi-objective optimization, where various metrics are used that typically observe different quality aspects have also been demonstrated [53].

Another major consideration is the choice of search method or optimiser. The selection of an appropriate method is typically conducted using experimentalism [20]. The utilized optimisers progressed from classic genetic algorithms [21] to proven faster methods such as differential evolution [59] and direct search derivative free methods [60]. For more complex optimization problems the use of metaheuristics are warranted over simpler search strategies [48]. Future implementations might select appropriate methods based on both experimentalism [20] and method performances on black 
box optimization benchmarks [19]. Parallel computing implementations for fitness evaluation processes such as segmentation have also been demonstrated that could reduce the required computing times [61].

A general objective in search centric sample supervised method research is to create methods that are either faster or leads to better quality solutions than current methods. The generalizability and adaptability performances of methods to problems have received little research attention. Some methods address problem complexity by incorporating algorithms to merge segments that are unable to be segmented correctly - irrespective of the tuning of the segmentation algorithm [62, 63]. Segmentation algorithms may also be defined that are more modular and thus better able to fit a range of problem instances [64, 65].

Another line of thinking that addresses the general drive to generate improved results suggests the creation of enlarged or higher dimensional search spaces. These enlarged search spaces not only consider or contain segmentation algorithm parameters, but also other parameterisable processes surrounding segmentation that might influence the results $[48,66]$. Data transformation functions or low-level image processing processes could be appended to the search spaces [43, 48], potentially resulting in better segmentation results. This notion has been extended to mathematical morphology inspired segmentation approaches, where attribute tuning, segmentation algorithm tuning and data transformation function tuning are combined in a single search space [65]. Metaheuristics could also be used to select the appropriate attributes in such approaches [67]. Another approach propose the selection, or searching of, mathematical morphology connected filters for a given problem [68].

Research methods surrounding sample supervised segment generation typically focus on comparing a new formulation against previous approaches in terms of achieved segment quality, required processing time and the robustness of results [23, 48, 69]. Generalisability performances and sampling size requirements may also be considered as performance indicators. Initial contributions focussed on proof-of-concept, commonly as conference papers (e.g. [21]), with more recent work (e.g. [48]) borrowing heavily from comparative research methods [20] from the optimisation community.

\subsection{Limitations of Search Centric Sample Supervised Segment Generation Approaches in GEOBIA}

Although the general technique of search centric sample supervised segment generation within GEOBIA shows promise, some limitations should be highlighted. A common problem or concern with a search centric sample supervised method is its ability to adapt to difficult or unseen problems [21]. A method might just be unable to address the given problem. Having expert knowledge of the capabilities of the used segmentation algorithm(s) could allow a user to judge the feasibility of the method beforehand. Another concern is the definition of a class of interest or the similarity of elements within a class. Thematically similar elements of interest might have vastly different spectral and geometric characteristics, suggesting that a segmentation algorithm may not be effectively tuned to solve such a problem [70]. For example, "buildings" in Figure 2.9 range from large rectangular bright objects to small grass roofed huts, suggesting multiple runs (sub-classes) of a method for a single class. 


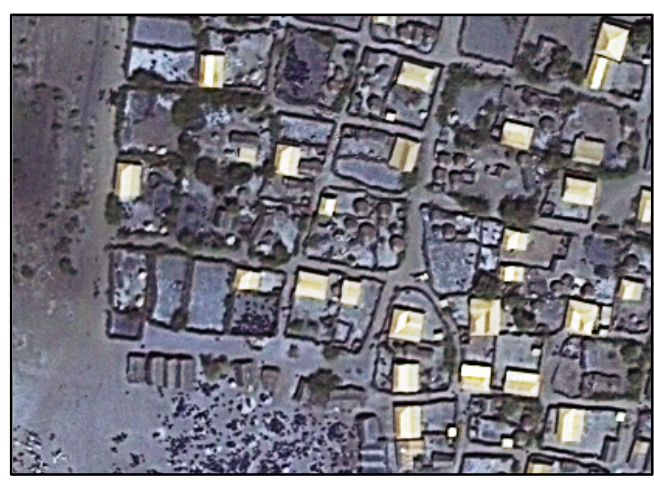

Figure 2.9. A segmentation scenario where the object of interest could be buildings of vastly different spectral and geometric characteristics.

The number of samples needed for such an approach to generalize well to the elements of interest has not been the topic of an investigation. Typically a small selection of reference samples are used in experimentation, with generalizability mechanisms such as cross-validation optional [48]. Methods from supervised classification could potentially be transferred to address such concerns [69, 71]. Another concern related to sampling is the processing time required and the extent of the areas that are segmented. More samples imply more processing time. Subsets of the image, centered on the reference segments can be extracted and used as input to a method, provided that segmentation results are identical compared to subsets (segmentation algorithm dependent).

Although various metrics have been used in such a general approach, little is known how metric values relate to final classification accuracies. It can be assumed that better quality segments leads to better quality classification results and information products. The utilized classification methods and attributes (features) should also be considered. Profiling the relations or correlations among used metrics and classifiers considering various (attribute) conditions might be fruitful. The value of different metrics or observed aspects or even of multi-objective optimization strategies might be better understood, specifically how the notions of under- and over-segmentation relate to classification results.

There are substantial uncertainties surrounding the usage and evaluation of metaheuristics. Although the general methods described here are considered applications of metaheuristics, the concerns raised in basic research should be noted. Metrics define the characteristics of the search landscape (e.g. Figure 2.2) and encoding or creating additional processes in the search landscape also inherently influences its characteristics [48]. Theory on how metaheuristics behave on various search landscapes are in its infancy $[13,72]$ with models able to predict problem difficulty a topic of active research [73]. In the context of search centric sample supervised segment generation approaches a handful of search methods have been experimentally profiled in terms of fitness traces over different search landscapes [48]. Simpler search methods such as a hill climber and random search displayed varied performances on a range of typical search landscapes [48], suggesting further investigations into the appropriateness of using specific search methods in these problem contexts.

Based on the uncertainties regarding performance theories and models, empirical research or experimentalism is generally advocated when conducting research on metaheuristic applications [13, $20,74]$. Such approaches typically require extensive experimentation to calculate measures of quality, 
robustness and generalizability, with numerous experimental runs required on various problem instances and under various conditions or parameter sets. Such considerations should be noted when conducting experimentation within image analysis and especially when considering segmentation, which is typically a computationally expensive process.

\subsection{An Outlook on Search Centric Sample Supervision in GEOBIA Approaches - Epistatic Links and Search Granularity}

In the context of GEOBIA and considering advances in image processing and metaheuristics, the question could be raised how one could define components of GEOBIA workflows as optimization problems. A current philosophy in rule set development suggest an expert user explores or investigates ways to obtain good results or build methods [10]. Efficient search methods could assist in such method construction or tuning processes. A very specific application of metaheuristics in GEOBIA is to tune the parameters of segmentation algorithms (as described above), but more encompassing or integrative implementations could be considered.

Classical GEOBIA processes such as attribution, segmentation, low-level image processing and classification [37] typically display high degrees of complex interactions [10] or high degrees of epistasis (dependencies) [75]. Such processes are parameterisable and their interactions could also be encoded in some manner. A simple example from the domain of machine learning suggests simultaneous classifier free parameter tuning and attribute subset selection [76]. Classification and attributes are not only dependent on each other (unless attribute selection is implicit in the classifier), but in all likelihood on the nature of the generated segments (segment parameters and metric scores). In addition, attributes themselves could be highly modular and designable [26] or tuneable [77]. The domain of mathematical morphology inspired image processing and analysis encompasses numerous highly modular, parameterisable or designable components related to segmentation (e.g.[78]).

Ways in which one could encode combined search problems from such varied components for efficient methods are unclear, although having different data quantizations in a single optimization problem are feasible [79]. Figure 2.10 illustrates an artificial or toy problem (Figure 2.10a) where the encoding of an arbitrary search centric sample supervised method consists of more elaborate parameter domains [66]. In this instance two classification processes (a one-class and a two class support vector machine, Figure $2.10 \mathrm{~b}, \mathrm{c}$ ) interacts with a data layering and segmentation process (Figure 2.10d) to aim to correctly segment and find the elements of interest.
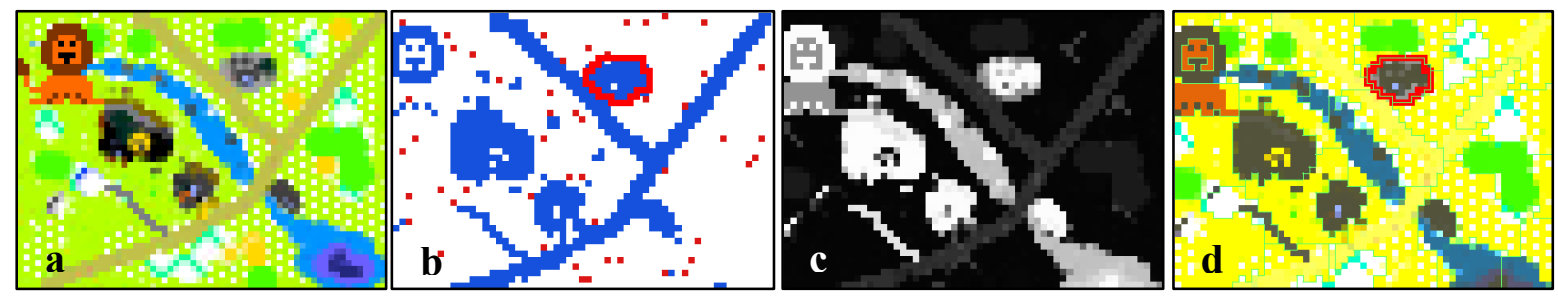
Figure 2.10. An arbitrary search centric image analysis method executed on a artificial problem (a) considering pixel-based classification processes $(b, c)$ and their interaction with data transformation and segmentation processes $(d)$.

Another point of contention is how such processes could elegantly integrate with existing GEOBIA workflows or philosophies (rule set development), and how good synergies between user experience/interaction and automatic search could be advocated. This is an important consideration in the context of emergency response or rapid mapping. In such scenarios information products need to be generated quickly. It is unclear if even the most advanced method designs could successfully address moderately difficult remote sensing image analysis problems on their own. A basic approach as depicted in Figure 2.6 would probably not deliver results of sufficient quality in most problem instances. In addition, there are numerous ways or intermediate steps to measure quality at the image processing and classification levels. These measures (or combinations of them) should correlate with user judgment. Another line of thinking suggests the creation of agent based modelling for rule-set adaptation [80].

The general processes described above and specifically the image processing could be considered as coarse grained processes. It is known that integrative approaches combining various aspects of image processing are needed to address complex image analysis problems [6, 81]. It would be interesting to see how fine grained (search centric) processes such as cellular automata [24] and genetic programming based image processing [8] would compare with segmentation algorithm parameter tuning considering the required search times, quality of results achieved and generalizability performances. Fine grained search centric methods might have interesting influences on, or synergies with traditional rule set based approaches, which currently only consider coarse grained parameter tuning processes with high generalizability performances.

\subsection{Conclusion}

Search centric sample supervised image analysis approaches might find use alongside traditional image analysis approaches within software environments, even if initially only to remove the guesswork from parameter tuning processes. Ultimately the involvement of a metaheuristic could range from simple parameter tuning to designing the entire image analysis method [14]. Although any mapping scenario might benefit from such strategies (e.g. land cover mapping), time critical mapping tasks (e.g. Copernicus Emergency Management Services) where user interaction is actively encouraged might benefit substantially. In such instances image analysis solutions (supervised classifiers, rule sets) to various problems can not readily be designed beforehand.

An overview of metaheuristics as tools for design was presented, with a focus on how such approaches have been applied within the domain of GEOBIA. Usage considerations and limitations of such a general approach were also highlighted. Based on developments from outside the domain of GEOBIA and acknowledging the unique processes within it, an outlook was given on potential avenues for future research, focusing on the concept of epistatic links among processes. How search centric sample supervised methods could integrate with current GEOBIA approaches to create efficient workflows should be a topic for future investigation. 
Currently search centric sample supervised approaches remain steadfastly within the domain of research, as performances are not sufficiently adequate (as of yet) and method nuisances are plentiful. Systematic investigations on the various aspects of search centric sample supervised methods will probably continue. Such approaches touch upon concepts from various academic disciplines or domains. In the case of GEOBIA and considering the current and potential designs of search centric sample supervised methods, concepts from machine learning, optimization, computer vision, image processing and remote sensing are involved (among others). This may also be evident based on the diversity of citations in this manuscript.

\subsection{References}

1. S.M. De Jong and F.D. Van Der Meer, Remote Sensing Image Analysis: Including the Spatial Domain, Springer, Dordrecht, 2004.

2. B. Jähne, Digital Image Processing, Springer, Berlin, 2005.

3. M.H.F. Wilkinson and J.B.T.M. Roerdink, Mathematical Morphology and Its Application to Signal and Image Processing, Springer, Berlin, 2009.

4. M. Pedergnana, P.R. Marpu, M.D. Mura, J.A. Benediktsson and L. Bruzzone, Classification of remote sensing optical and lidar data using extended attribute profiles, IEEE Journal of Selected Topics in Signal Processing, 6, 856-865, (2012).

5. K. Mikolajczyk and C. Schmid, A performance evaluation of local descriptors, Pattern Analysis and Machine Intelligence, IEEE Transactions on, 27, 1615-1630, (2005).

6. S. Zheng, A. Yuille and Z. Tu, Detecting object boundaries using low-, mid-, and high-level information, Computer Vision and Image Understanding, 114, 1055-1067, (2010).

7. R. Datta, D. Joshi, J. Li and J.Z. Wang, Image retrieval: Ideas, influences, and trends of the new age, ACM Computing Surveys, 40, Article 5, (2012).

8. S. Harding, J. Leitner and J. Schmidhuber, Cartesian genetic programming for image processing, in Genetic Programming Theory and Practice X, R. Riolo, E. Vladislavleva, M.D. Ritchie and J.H. Moore (Eds), Springer, New York, 2013.

9. T. Blaschke, G.J. Hay, M. Kelly, S. Lang, P. Hofmann, E. Addink, R. Queiroz Feitosa, F. Van Der Meer, H. Van Der Werff, F. Van Coillie and D. Tiede, Geographic Object-Based Image Analysis - Towards a new paradigm, ISPRS Journal of Photogrammetry and Remote Sensing, 87, 180-191, (2014).

10. M. Baatz, C. Hoffman and G. Willhauck, Progressing from object-based to object-oriented image analysis, in Object-Based Image Analysis: Spatial Concepts for Knowledge-Driven Remote Sensing Applications, T. Blaschke, S. Lang and G.J. Hay (Eds), Springer, Berlin, 2008.

11. E. Talbi, Metaheuristics: From Design to Implementation, Wiley, Hoboken, 2009.

12. S.J. Louis, Genetic Algorithms as a Computational Tool for Design, Doctor of Philosophy, Indiana University, 1993.

13. L. Vanneschi, L. Mussi and S. Cagnoni, Hot topics in evolutionary computation, Intelligenza Artificiale, 5, 5-17, (2011).

14. S. Cagnoni, Evolutionary computer vision: a taxonomic tutorial, Hybrid Intelligent Systems (HIS 2008), 10-12 September, Barcelona, Spain, 2008. 
15. S. Cagnoni, E. Lutton and G. Olague, Genetic and Evolutionary Computation for Image Processing and Analysis, Hindawi Publishing Corporation, New York, 2007.

16. S. Walton, O. Hassan, K. Morgan and M. Brown, Modified cuckoo search: a new gradient free optimisation algorithm, Chaos, Solitons \& Fractals, 44, 710-718, (2011).

17. K.V. Price, R.M. Storn and J.A. Lampinen, Differential Evolution: A Practical Approach to Global Optimization, Springer, Berlin, 2005.

18. B. Bhanu, S. Lee and J. Ming, Adaptive image segmentation using a genetic algorithm, Systems, Man and Cybernetics, IEEE Transactions on, 25, 1543-1567, (1995).

19. N. Hansen, A. Auger, S. Finck and R. Ros, Real-Parameter Black-Box Optimization Benchmarking 2010: Experimental Setup, INRIA Research Report RR-7215, 2010.

20. T. Bartz-Beielstein, Experimental Research in Evolutionary Computation, Springer, Berlin, Germany, 2006.

21. R.Q. Feitosa, G.a.O.P. Costa, T.B. Cazes and B. Feijo, A genetic approach for the automatic adaptation of segmentation parameters, in Geographic Object-Based Image Analysis (GEOBIA 2006), S. Lang, T. Blaschke and E. Schoepfer (Eds), ISPRS, Salzburg, 2006.

22. G. Pignalberi, R. Cucchiara, L. Cinque and S. Levialdi, Tuning range image segmentation by genetic algorthm, EURASIP Journal on Applied Signal Processing, Volume 2003, 780-790, (2003).

23. S. Derivaux, G. Forestier, C. Wemmert and S. Lefevre, Supervised image segmentation using watershed transform, fuzzy classification and evolutionary computation, Pattern Recognition Letters, 31, 2364-2374, (2010).

24. I. Yoda, K. Yamamoto and H. Yamada, Automatic acquisition of hierarchical mathematical morphology procedures by genetic algorithms, Image and Vision Computing, 17, 749-760, (1999).

25. P.L. Rosin, Image processing using 3-state cellular automata, Computer Vision and Image Understanding, 114, 790-802, (2010).

26. T. Kowaliw, W. Banzhaf, N. Kharma and S. Harding, Evolving novel image features using genetic programming-based image transforms, in Evolutionary Computation (CEC'09), IEEE Congress on, IEEE, 2502-2507, Trondheim, Norway, 2009.

27. B. Bhanu and J. Peng, Adaptive integrated image segmentation and object recognition, IEEE Transactions on Systems, Man, and Cybernetics, 30, 427-441, (2000).

28. V. Martin and M. Thonnat, A cognitive vision approach to image segmentation, Tools in Artificial Intelligence, 1, 265-294, (2008).

29. M. Ebner, A real-time evolutionary object recognition system, in Genetic Programming, L. Vanneschi, S. Gustafson, A. Moraglio, I. De Falco and M. Ebner (Eds), Springer, Berlin, 2009.

30. Y.J. Zhang, A survey on evaluation methods for image segmentation, Pattern Recognition, 29, 1335-1346, (1996).

31. S. Chabrier, B. Emile, C. Rosenberger and H. Laurent, Unsupervised performance evaluation of image segmentation, EURASIP Journal on Applied Signal Processing, Volume 2006, 1-12, (2006). 
32. C. Kurtz, N. Passat, P. Gancarski and A. Puissant, Extraction of complex patterns from multiresolution remote sensing images: A hierarchical top-down methodology, Pattern Recognition, 45, 685-706, (2012).

33. T. Blaschke, Object based image analysis for remote sensing, ISPRS Journal of Photogrammetry and Remote Sensing, 65, 2-16, (2010).

34. E.A. Addink, F.M.B. Van Coillie and S.M. De Jong, Introduction to the GEOBIA 2010 special issue: From pixels to geographic objects in remote sensing image analysis, International Journal of Applied Earth Observation and Geoinformation, 15, 1-6, (2012).

35. G.J. Hay and G. Castilla, Geographic object-based image analysis (GEOBIA): A new name for a new discipline, in Object-Based Image Analysis: Spatial Concepts for Knowledge-Driven Remote Sensing Applications, T. Blaschke, S. Lang and G.J. Hay (Eds), Springer, Berlin, Germany, 2008.

36. G. Castilla and G.J. Hay, Image objects and geographic objects, in Object-Based Image Analysis: Spatial Concepts for Knowledge-Driven Remote Sensing Applications, T. Blaschke, S. Lang and G.J. Hay (Eds), Springer, Berlin, Germany, 2008.

37. T. Lübker and G. Schaab, A work-flow design for large-area multilevel GEOBIA: Integrating statistical measures and expert knowledge, in Geographic Object-Based Image Analysis (GEOBIA 2010), E.A. Addink and F.M.B. Van Coillie (Eds), ISPRS, Ghent, Belgium, 2010.

38. L. Drăguţ, D. Tiede and S.R. Levick, ESP: a tool to estimate scale parameter for multiresolution image segmentation of remotely sensed data, International Journal of Geographical Information Science, 24, 859-871, (2010).

39. J. Kersten, M. Gähler and S. Voigt, A general framework for fast and interactive classification of optical VHR satellite imagery using hierarchical and planar Markov random fields, Photogrammetrie, Fernerkundung, Geoinformation, Volume 2010, 439-449, (2010).

40. C. Doulaverakis, A. Tzotsos, I. Tsampoulatidis, N. Gerontidis, A. Argyridis, K. Karantzalos, I. Kompatsiaris and D. Argialas, Gnorasi: A modular knowledge-based platform for object-based image analysis, South-Eastern European Journal of Earth Observation and Geomatics, 3, 473-476, (2014).

41. D. Passo, E. Bias, R. Brites and G. Costa, Comparison of the accuracy of classifications generated by interimage and by interimage integrated with data mining, South-Eastern European Journal of Earth Observation and Geomatics, 3, 93-97, (2014).

42. G.J. Hay, G. Castilla, M.A. Wulder and J.R. Ruiz, An automated object-based approach for the multiscale image segmentation of forest scenes, International Journal of Applied Earth Observation and Geoinformation, 7, 339-359, (2005).

43. C. Fourie and E. Schoepfer, Combining the heuristic and spectral domains in semi-automated segment generation, Geographic Object-based Image Analysis (GEOBIA 2012), 7-9 May 2012, Brazil, 2012.

44. H. Herold and G. Meinel, Adaptive Segmentation of Heterogeneous Images Using Metaheuristic Evolutionary Optimization, South-Eastern European Journal of Earth Observation and Geomatics, 3, 461-464, (2014).

45. J. Osman, J. Inglada and E. Christophe, Interactive object segmentation in high resolution satellite images, IEEE International Geoscience and Remote Sensing Symposium, 12-17 July 2009, Cape Town, 2009. 
46. F. Van Coillie, L.P. Verbeke and R.R. De Wulf, Feature selection by genetic algorithms in objectbased classification of IKONOS imagery for forest mapping in Flanders, Belgium, Remote Sensing of Environment, 110, 476-487, (2007).

47. C. Fourie, A One Class Object Based System for Sparse Geographic Feature Identification, M.Sc., Stellenbosch University, 2011.

48. C. Fourie and E. Schoepfer, Data transformation functions for expanded search spaces in geographic sample supervised segment generation, Remote Sensing, 5, 3791-3821, (2014).

49. P. Achanccaray, V. Ayma, L. Jimenez, S. Garcia, P. Happ, R. Feitosa and A. Plaza, A free software tool for automatic tuning of segmentation parameters, South-Eastern European Journal of Earth Observation and Geomatics, 3, 707-712, (2014).

50. A. Carleer, O. Debeir and E. Wolff, Assessment of very high spatial resolution satellite image segmentations, Photogrammetric Engineering and Remote Sensing, 71, 1285-1294, (2005).

51. Q. Zhan, M. Molenaar, K. Tempfli and W. Shi, Quality assessment for geo-spatial objects derived from remotely sensed data, International Journal of Remote Sensing, 26, 2953-2974, (2005).

52. U. Weidner, Contribution to the assessment of segmentation quality for remote sensing applications, International Archives of Photogrammetry, Remote Sensing and Spatial Information Sciences, 37, 479-484, (2008).

53. C. Persello and L. Bruzzone, A novel protocol for accuracy assessment in classification of very high resolution images, Geoscience and Remote Sensing, IEEE Transactions on, 48, 1232-1244, (2010).

54. B. Özdemir, S. Aksoy, S. Eckert, M. Pesaresi and D. Ehrlich, Performance measures for object detection evaluation, Pattern Recognition Letters, 31, 1128-1137, (2010).

55. R.Q. Feitosa, R.S. Ferreira, C.M. Almeida, F.F. Camargo and G.a.O.P. Costa, Similarity metrics for genetic adaptation of segmentation parameters, in Geographic Object-Based Image Analysis (GEOBIA 2010), E. Addink and F. Van Coillie (Eds), ISPRS, Ghent, Belgium, 2010.

56. P. Corcoran, A. Winstanley and P. Mooney, Segmentation performance evaluation for object-based remotely sensed image analysis, International Journal of Remote Sensing, 31, (2010).

57. M. Neubert, H. Herold and G. Meinel, Evaluation of remote sensing image segmentation qualityfurther results and concepts, International Archives of Photogrammetry, Remote Sensing and Spatial Information Sciences, 36, (2006).

58. R. Trias-Sanz, G. Stamon and J. Louchet, Using colour, texture, and hierarchical segmentation for high-resolution remote sensing, ISPRS Journal of Photogrammetry and Remote Sensing, 63, 156168, (2008).

59. L.M. Melo, G.a.O.P. Costa, R.Q. Feitosa and A.V. Da Cruz, Quantum-inspired evolutionary algorithm and differential evolution used in the adaptation of segmentation parameters, in Geographic Object-Based Image Analysis (GEOBIA 2008), G.J. Hay, T. Blaschke and D. Marceau (Eds), ISPRS, 6-7, Calgary, Canada, 2008.

60. P. Happ, R.Q. Feitosa and A. Street, Assessment of optimization methods for automatic tuning of segmentation parameters, Geographic Object-based Image Analysis (GEOBIA 2012), 7-9 May 2012, Rio De Janeiro, Brazil, 2012.

61. P. Happ, R. Feitosa, C. Bentes and R. Farias, A Region-Growing Segmentation Algorithm for GPUs, IEEE Geoscience and Remote Sensing Letters, 10, 1612-1616, (2013). 
62. C.M.B. Freddrich and R.Q. Feitosa, Automatic adaptation of segmentation parameters applied to non-homogeneous object detection, in Geographic Object-based Image Analysis (GEOBIA 2008), G.J. Hay, T. Blaschke and D. Marceau (Eds), ISPRS, Calgary, Canada, 2008.

63. J. Michel, M. Grizonnet and O. Canevet, Supervised re-segmentation for very high-resolution satellite images, Geoscience and Remote Sensing Symposium (IGARSS 2012), IEEE International, 22-27 July 2012, Munich, Germany, 2012.

64. R.S. Ferreira, R.Q. Feitosa and G.a.O.P. Costa, A multiscalar, multicriteria approach for image segmentation, Geographic Object-Based Image Analysis (GEOBIA 2012), 7-9 May 2012, Rio de Janeiro, Brazil, 2012.

65. C. Fourie and E. Schoepfer, Connectivity Thresholds and Data Transformations in Sample Supervised Segment Generation, Geoscience and Remote Sensing Symposium (IGARSS 2012), IEEE International, 21-26 July, Melbourne, 2013.

66. C. Fourie and E. Schoepfer, Classifier Directed Data Transformations in Sample Supervised Segment Generation, Geographic Object-based Image Analysis (GEOBIA 2014), 21-24 May, Thessaloniki, Greece, 2014.

67. M. Pedergnana, P.R. Marpu, M. Dalla Mura, J.A. Benediktsson and L. Bruzzone, A novel technique for optimal feature selection in attribute profiles based on genetic algorithms, IEEE Transactions on Geoscience and Remote Sensing, 51, 3514-3528, (2013).

68. M. Dalla Mura, J.A. Benediktsson and L. Bruzzone, A general approach to the spatial simplification of remote sensing images based on morphological connected filters, Geoscience and Remote Sensing Symposium (IGARSS 2012), IEEE International, 24-29 July, Vancouver, Canada, 2011.

69. G.M. Foody and A. Mathur, A relative evaluation of multiclass image classification by support vector machines, IEEE Transactions on Geoscience and Remote Sensing, 42, 1335-1343, (2004).

70. M. Musci, R.Q. Feitosa and G.a.O.P. Costa, An object-based image analysis approach based on independent segmentations, Joint Urban Remote Sensing Event, 21-23 April, Sao Paulo, Brazil, 2013.

71. M. Birattari, M. Zlochin and M. Dorigo, Towards a theory of practice in metaheuristics design: A machine learning perspective, RAIRO-Theoretical Informatics and Applications, 40, 353-369, (2006).

72. J. Watson, An introduction to fitness landscape analysis and cost models for local search, in Handbook of Metaheuristics, M. Gendreau and J. Potvin (Eds), Springer US, 2010.

73. M. Graff and R. Poli, Practical performance models of algorithms in evolutionary program induction and other domains, Artificial Intelligence, 174, 1254-1276, (2010).

74. R.L. Rardin and R. Uzsoy, Experimental Evaluation of Heuristic Optimization Algorithms: A Tutorial, Journal of Heuristics, 7, 261-304, (2001).

75. K. Weicker and N. Weicker, On the improvement of coevolutionary optimizers by learning variable interdependencies, Evolutionary Computation (CEC 1999), 6-9 July 1999, Washington DC, United States of America, 1999.

76. S.-W. Lin, K.-C. Ying, S.-C. Chen and Z.-J. Lee, Particle swarm optimization for parameter determination and feature selection of support vector machines, Expert Systems with Applications, 35, 1817-1824, (2008). 
77. T. Ojala, M. Pietikainen and T. Maenpaa, Multiresolution gray-scale and rotation invariant texture classification with local binary patterns, Pattern Analysis and Machine Intelligence, IEEE Transactions on, 24, 971-987, (2002).

78. G.K. Ouzounis and M.H. Wilkinson, Hyperconnected attribute filters based on k-flat zones, Pattern Analysis and Machine Intelligence, IEEE Transactions on, 33, 224-239, (2011).

79. J. Lampinen and I. Zelinka, Mixed integer-discrete-continuous optimization by differential evolution, in 5th International Conference on Soft Computing, Citeseer, 77-81, 1999.

80. P. Hofmann, P. Lettmayer, T. Blaschke, M. Belgiu, S. Wegenkittl, R. Graf, T. Lampoltshammer and V. Andrejchenko, ABIA - A Conceptual Framework for Agent Based Image Analysis, SouthEastern European Journal of Earth Observation and Geomatics, 3, 125-130, (2014).

81. C. Li, A. Kowdle, A. Saxena and T. Chen, Toward holistic scene understanding: Feedback enabled cascaded classification models, Pattern Analysis and Machine Intelligence, IEEE Transactions on, 34, 1394-1408, (2012). 


\title{
CHAPTER 3: DATA TRANSFORMATION FUNCTIONS FOR EXPANDED SEARCH SPACES IN GEOGRAPHIC SAMPLE SUPERVISED SEGMENT GENERATION
}

\author{
Fourie, C \& Schoepfer, E \\ German Remote Sensing Data Center (DFD), German Aerospace Center (DLR), \\ 82234 Oberpfaffenhofen.
}

Published in:

Remote Sensing (2014), 5, 3791-3821

Received: 30 January 2014; in revised form: 28 March 2014 / Accepted: 16 April 2014 / Published: 28 April 2014 doi:10.3390/rs6053791 
Article

\title{
Data Transformation Functions for Expanded Search Spaces in Geographic Sample Supervised Segment Generation
}

\author{
Christoff Fourie * and Elisabeth Schoepfer
}

German Remote Sensing Data Center (DFD), German Aerospace Center (DLR), 82234 Oberpfaffenhofen, Germany; E-Mail: elisabeth.schoepfer@dlr.de

* Author to whom correspondence should be addressed; E-Mail: christoffel.fourie@dlr.de; Tel.: +49-81-5328-1383; Fax: +49-81-5328-1445.

Received: 30 January 2014; in revised form: 28 March 2014 / Accepted: 16 April 2014 /

Published: 28 April 2014

\begin{abstract}
Sample supervised image analysis, in particular sample supervised segment generation, shows promise as a methodological avenue applicable within Geographic Object-Based Image Analysis (GEOBIA). Segmentation is acknowledged as a constituent component within typically expansive image analysis processes. A general extension to the basic formulation of an empirical discrepancy measure directed segmentation algorithm parameter tuning approach is proposed. An expanded search landscape is defined, consisting not only of the segmentation algorithm parameters, but also of low-level, parameterized image processing functions. Such higher dimensional search landscapes potentially allow for achieving better segmentation accuracies. The proposed method is tested with a range of low-level image transformation functions and two segmentation algorithms. The general effectiveness of such an approach is demonstrated compared to a variant only optimising segmentation algorithm parameters. Further, it is shown that the resultant search landscapes obtained from combining mid- and low-level image processing parameter domains, in our problem contexts, are sufficiently complex to warrant the use of population based stochastic search methods. Interdependencies of these two parameter domains are also demonstrated, necessitating simultaneous optimization.
\end{abstract}

Keywords: geographic object-based image analysis; segmentation; data transformations; sample supervised; spatial metrics; metaheuristics 


\subsection{Introduction}

A general method in the context of geographic sample supervised segment generation is proposed and profiled. Parameter interdependencies are noted between low- and mid-level image processing processes. Creating combined search spaces using algorithms from these two groupings are proposed that could lead to improved segmentation results. In this section an orientation to the problem of semantic segmentation within Geographic Object-based Image Analysis (GEOBIA) is given and empirical discrepancy method based supervised segment generation approaches that address this problem are reviewed, to which our method also belongs. Motivation for the proposed method is presented, with details on how it extends the general sample supervised segment generation approach.

\subsubsection{Semantic Segmentation in Geographic Object-Based Image Analysis}

Geographic Object-based Image Analysis (GEOBIA) [1-4] has garnered interest by practitioners and researchers alike as an effective avenue of methods, based on the principle of semantic segmentation, to address specific remote sensing image analysis problems. The increased spatial resolution of optical Earth observation imagery to below the sub-decimeter level and a swath of new applications highlighted the inefficiency of traditional remote sensing image analysis techniques (per-pixel methods) not addressing scale-space considerations [5-7]. In the context of object-based image analysis, semantic image partitioning or segmentation is a common constituent of approaches that attempt to create a more meaningful or workable representation of the data $[2,3]$. Common other constituents in GEOBIA approaches include attribution (feature description), classification (supervised and expert system's approaches), and information representation, with potentially complex interactions among them [8], and potentially differing levels of supervision [9].

Popular variants of segmentation algorithms (e.g., region-merging and region-growing) used within the context of GEOBIA may have controlling mechanisms or parameters dictating the relative sizes and geometric characteristics of generated segments [10,11]. A segmentation process could either be performed with the aim of creating a single segment layer addressing a specific problem, or as part of a hierarchical image analysis approach [12]. In many problem instances it could be feasible to segment some elements of interest with a single pass of a segmentation algorithm, due to the similar geometric characteristics of these elements. In the context of multi-scale analysis approaches, the aim could be to identify appropriate scale-space representations that would ease subsequent post-segmentation element identification processes.

Finding a suitable segmentation algorithm parameter set and resulting segments is an important aspect of the analysis procedure with a definite influence on subsequent results. It is not uncommon for parameter tuning to be conducted in a user driven empirical trial-and-error process, which is labor intensive and could fail to obtain optimal results. In image analysis disciplines this subjectivity has driven research into various approaches that aim to evaluate the quality of segments based on some given quality criteria. Such approaches could be grouped into two broad categories based on how an indication of quality is given [13].

Empirical goodness methods [13] encode human intuition or notions of quality (e.g., shape, intra region uniformity, statistical properties) in the evaluation procedure without any a priori information on correct segmentation. An example of such an approach is using local variance to define layers in 
multi-scale image segmentation [7]. Empirical discrepancy methods [13] requires information regarding correct segmentation, typically in the form of reference segments provided by a user judged to be representative of the desired output. These reference segments are compared, based on geometric and/or spectral characteristics encoded into metrics, with generated segments to give an indication of quality. It could be argued that empirical discrepancy methods synergize well with many user-driven or expert system's GEOBIA approaches, as a user is readily available to give examples or indicators of quality during the method development or even during the execution phase.

\subsubsection{Sample Supervised Segment Generation}

Sample supervised segment generation describes methods that aim to automatically generate good quality segments based on limited reference segment examples [14]. Commonly empirical discrepancy methods are used to measure quality and drive a search process to find a good segmentation set from potentially many generated results [15-17]. Such approaches are typically iterative methods that search in a directed or undirected manner for good results. Due to the computationally expensive nature of image segmentation, emphasis is often placed on searching for good results efficiently, typically by using and experimentally evaluating [18] metaheuristics for this purpose. Sample supervised segment generation can be seen as an explicit example of more general approaches found at the intersection of evolutionary computation and image analysis/computer vision [19]. A distinction can be made [14] based on the granularity of the search process - whether the search method is used to construct a segmentation algorithm/image processing method [20-24], common with cellular automata, mathematical morphology and genetic programming approaches, or either for tuning the free parameters of an algorithm [14,25-28].

The free parameter tuning approach has been investigated in the context of Very High Resolution (VHR) remote sensing optical image analysis problems [25,29], where the parameter space of a popular segmentation algorithm is searched [10]. In this context such an approach shows promise with segmentation algorithms that generalize well. None the less the approach is still very sensitive to numerous considerations, such as the quality and quantity of the given reference segments, image characteristics, and the intrinsic ability of the segmentation algorithm to reach the desired reference segments [25] (which is difficult to determine a priori), the choice of empirical discrepancy method [15], and the capabilities of the utilized search method [30].

The intrinsic ability of the segmentation algorithm to achieve the desired results is one of the most challenging concerns. For illustration, when using an algorithm strongly constrained in the scale-space, land-cover-elements of varying size cannot be segmented correctly using a single segmentation layer, irrespective of how the algorithm is tuned. Another scenario common in the context of satellite image analysis could be that the elements of interest are geometrically similar, but deviations in their spectral characteristics prevent adequate segmentation. This could pose a problem for a segmentation algorithm strongly observing spectral characteristics. Some approaches address this issue by introducing automatic post-segmentation procedures to merge over-segmented objects [31,32]. More commonly such problems are addressed with expert systems or rule set approaches where segments are split and merged depending on the given problem [8]. 


\subsubsection{Integrative Approaches to Image Analysis}

Due to the shortcomings of image segmentation approaches to detect semantic objects in complex natural images as described above, various approaches have been proposed that combine cues from different image analysis methods. Such strategies commonly attempt to combine low-, mid-, and high-level image processing processes, with the processes functioning independently or having some form of communication or information exchange among them [33-40]. Often an interaction between template matching (high-level process) and semantic segmentation (mid-level process) is advocated. Similarly within the domain of classification, approaches have been put forth that consider different aspects of a methodology simultaneously due to interactions among the constituent parts, for example simultaneous attribute selection and classifier free parameter tuning [41-43].

In the context of image processing, another line of work explores the effect of data representations or color spaces on image analysis tasks, also within the domain of remote sensing [44-47]. This constitutes the selection or creation of low-level image processing where the data is transformed to another representation or color space that is more suited to the given problem. In [46] different color spaces are created and evaluated quantitatively for the task of a multi-scale segmentation of aerial images using sequential forward selection. Low-level processes are sometimes also modelled as optimization problems [48,49]. These low-level image processing data transformation approaches do not consider the interaction between the data transformations and subsequent processes.

\subsubsection{Combined Low- and Mid-Level Image Processing Optimization for Geographic Sample Supervised Segment Generation}

In the context of sample supervised segment generation (Section 3.1.2) and inspired by integrative approaches to image analysis and work on data transformations as described in Section 3.1.3, a general approach is proposed in this work that models the generation of quality segments as an optimization problem integrating low- and mid-level image processing. Low-level parameterized image processing methods, such as in $[48,49]$, are combined with parameterized image segmentation algorithms (e.g., [10]) to form an expanded parameter search space. This search space is traversed iteratively with a metaheuristic to find the optimal combined parameter set, consisting of the parameters of the low-level process as well as the parameters for the segmentation algorithm. It is shown that interdependencies [50,51] exists between these two parameter domains. In the context of this approach a wide range of combinations of low- and mid-level processes are possible, resulting in higher dimensional search spaces and being slightly more computationally expensive than a segmentation parameter tuning only approach, but with the potential to generate markedly improved segmentation results.

The initial concept was first suggested by the authors as a conference contribution in [52]. Here, the investigation is extended by testing the generalizability performance of the method, also using a range of metrics and performing more exhaustive experimental runs, investigating the performances and applicability of different search methods, testing additional promising data transformation functions, and illustrating parameter domain interdependencies.

In Section 3.2 an overview of the general method is given with details on its constituent parts. Section 3.3 describes the data, representative of common problems such a method could assist in addressing. In Section 3.4 the evaluation and profiling methodologies are described, with results and discussions 
presented in Section 3.5. Section 3.6 concludes by highlighting some shortcomings and open questions for further research.

\subsection{Expanded Search Spaces in Sample Supervised Segment Generation}

\subsubsection{Method Overview}

The method described here follows the general architecture of empirical discrepancy metric guided optimization-based methods [14,25,26]. Figure 3.1 illustrates the architecture of the variant presented in this work. The main contribution lies in the introduction of low-level image processing in the optimization loop, increasing the dimensionality of the search space. The method can be broken down into three distinct components. A user input component, the optimization loop, which constitutes the main body of the method and the output component.

The input component requires a user to provide input imagery and a selection of reference segments representative of the elements of interest. In our implementation such input data are handled separately with the reference segments delineated in a binary raster image, although sharing the metadata of the input image. As a preprocessing step, subsets are extracted from the input image and reference segments image over the areas where reference segments are provided. In Figure 3.1, such an image subset and corresponding reference segment subset is illustrated under the input component. It should be noted that the method takes as input all created subsets (a subset stack), not just those of a single element as depicted in Figure 3.1 for simplicity. The collection of input image and reference segments subsets are used within the optimization loop.

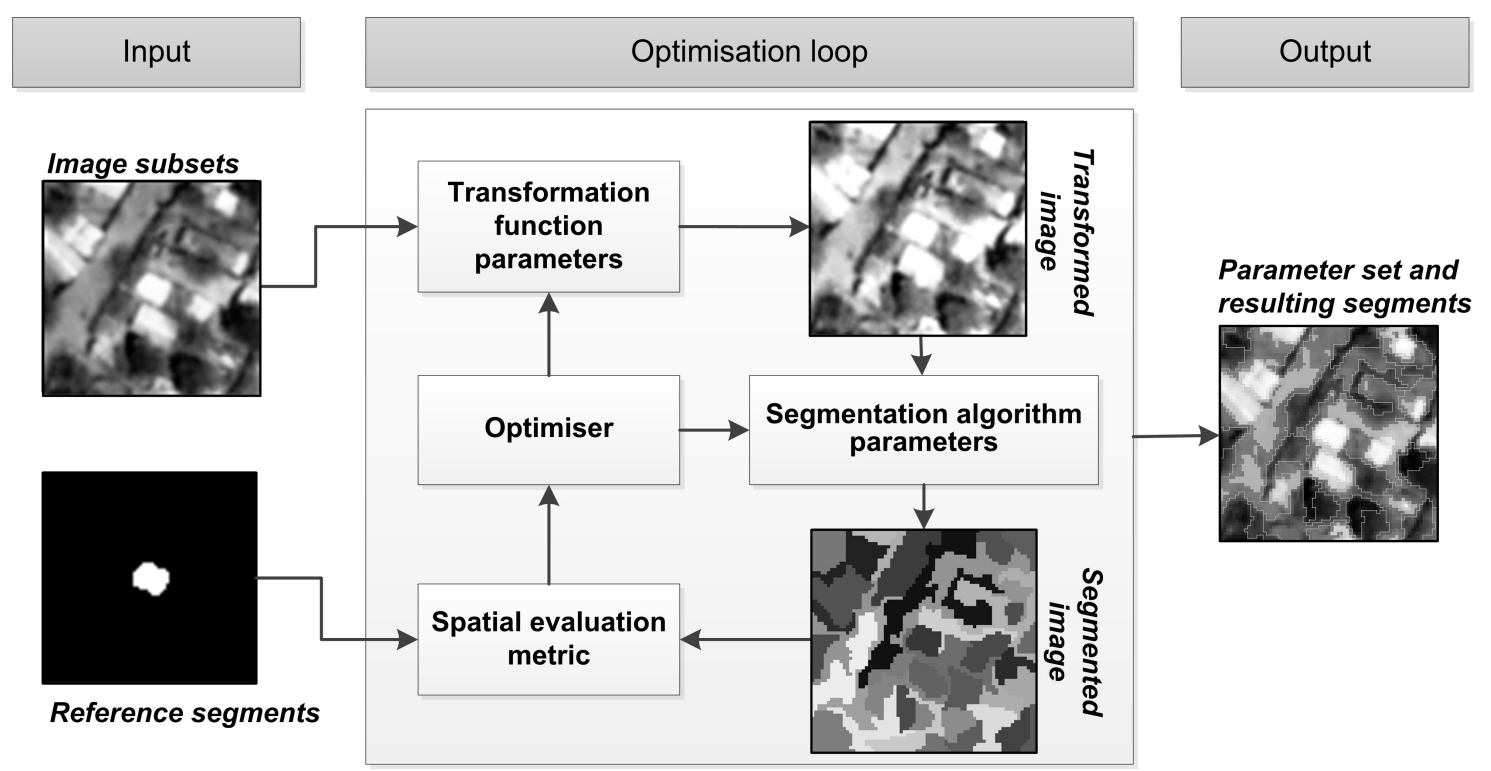

Figure 3.1. The method architecture for sample supervised segment generation with added data transformation functions.

The optimization loop consists of an efficient search method traversing two parameter domains simultaneously and receiving feedback from a spatial metric (empirical discrepancy method). The search method used here is detailed in Section 3.2.2. Figure 3.1 illustrates the interacting processes at iterations of the optimization loop. Firstly a parameter set is transferred from the optimizer to a given 
data transformation function (low-level image processing process). This data transformation function, with the provided parameter values, is invoked on the input image subsets returning transformed image subsets. A range of low-level image transformation functions are tested with this method, detailed in Section 3.2.3.

A second parameter set is passed on from the optimizer to the given segmentation algorithm. The segmentation algorithm with its provided parameter values is invoked on the transformed image subsets, resulting in a stack of segmented images (as in Figure 3.1). The segmentation algorithms used in this work are detailed in Section 3.2.4. Finally a spatial evaluation metric is invoked, taking as input the reference segments subsets (under "input") and the segmented image subsets. The final score given by the evaluation metric is the average over all provided reference segment subset and segmented image subset pairs. In the context of using metaheuristics such a metric is also referred to as an objective or fitness function. This score is passed on to the optimizer, which uses the information to direct the search in the next iteration. A range of single-objective evaluation metrics are tested in this work, detailed in Section 3.2.5. In Section 3.2.6 some example segmentation results are highlighted, with different parameters from the segmentation algorithm and data transformation domains and accompanying metric scores.

The method terminates after a certain number of user defined search iterations have passed, but various other stopping strategies are possible. As an output the parameter set resulting in the best segmentations are given, as judged by the evaluation metric. Note that during the search process processing is only done on the small image subsets to reduce computing time. After the method terminates the output (parameter set) can be used to segment the whole image or other images. Cross-validation and averaging strategies may help to ensure generalizability.

\begin{tabular}{|c|c|c|c|c|c|c|c|}
\hline \multicolumn{8}{|c|}{ Example encoding of a parameter set } \\
\hline Domain: & \multicolumn{4}{|c|}{ Data transformation function parameters } & \multicolumn{3}{|c|}{ Segmentation algorithm parameters } \\
\hline Description: & Band Position 1 & Band Position.. & Band Position n & Height & Scale & Shape/Colour & $\begin{array}{c}\text { Compactness/ } \\
\text { Smoothness }\end{array}$ \\
\hline Range: & {$[0,255]$} & {$[0,255]$} & {$[0,255]$} & {$[0,60]$} & {$[5,70]$} & {$[0,1]$} & {$[0,1]$} \\
\hline
\end{tabular}

Figure 3.2. An example encoding of a parameter set traversed by a given search algorithm, highlighting the creation of a joint set from the segmentation algorithm and a low-level image processing function.

Figure 3.2 illustrates an example encoding or parameter space that is traversed by the search algorithm. In this example the parameter space consists of a four parameter data transformation function (Spectral Split, detailed in Section 3.2.3) and a three parameter segmentation algorithm (Multiresolution Segmentation (MS), detailed in Section 3.2.4) resulting in a combined seven dimensional search space. The value ranges of the parameters are also shown. In our implementation all quantizations for parameters are converted to real values, due to the use of a real-valued optimizer. Figure 3.3 illustrates a two-dimensional exhaustive calculation of fitness of the seven dimensional parameter space from Figure 3.2 on an arbitrary problem and metric, highlighting multimodalities or interdependencies between two parameters from the two different parameter domains. Lower values suggest better segmentation results. 


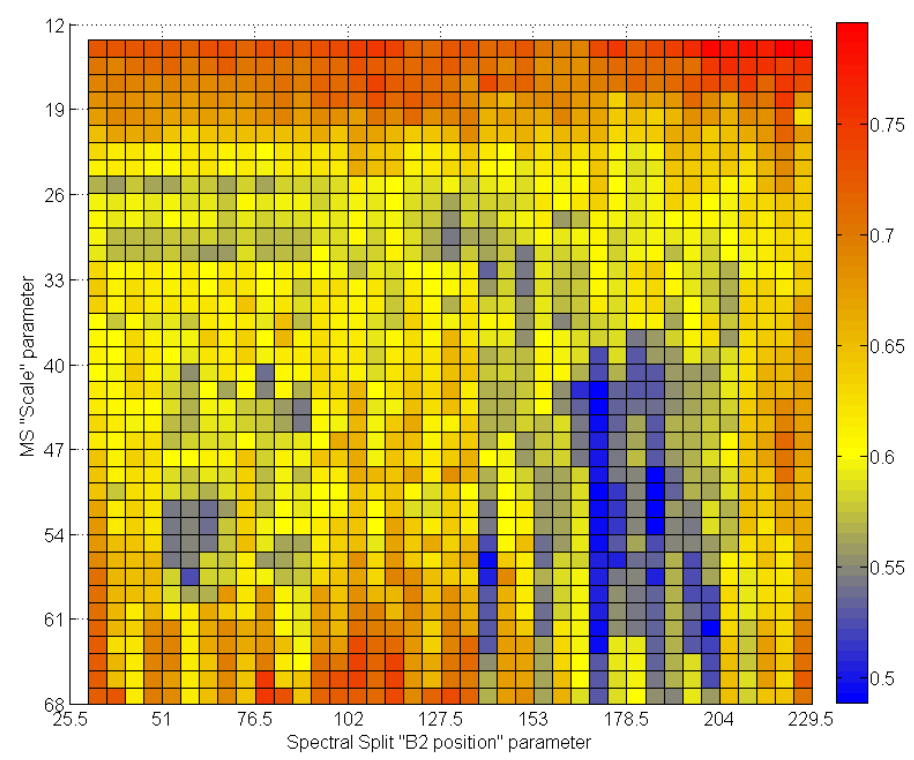

Figure 3.3. Two dimensions (band position 1 and scale parameters) of the seven dimensional search space of the parameter set depicted in Figure 3.2 (average of 15 reference segments) using the Reference Weighted Jaccard (RWJ) metric (Section 3.2.5).
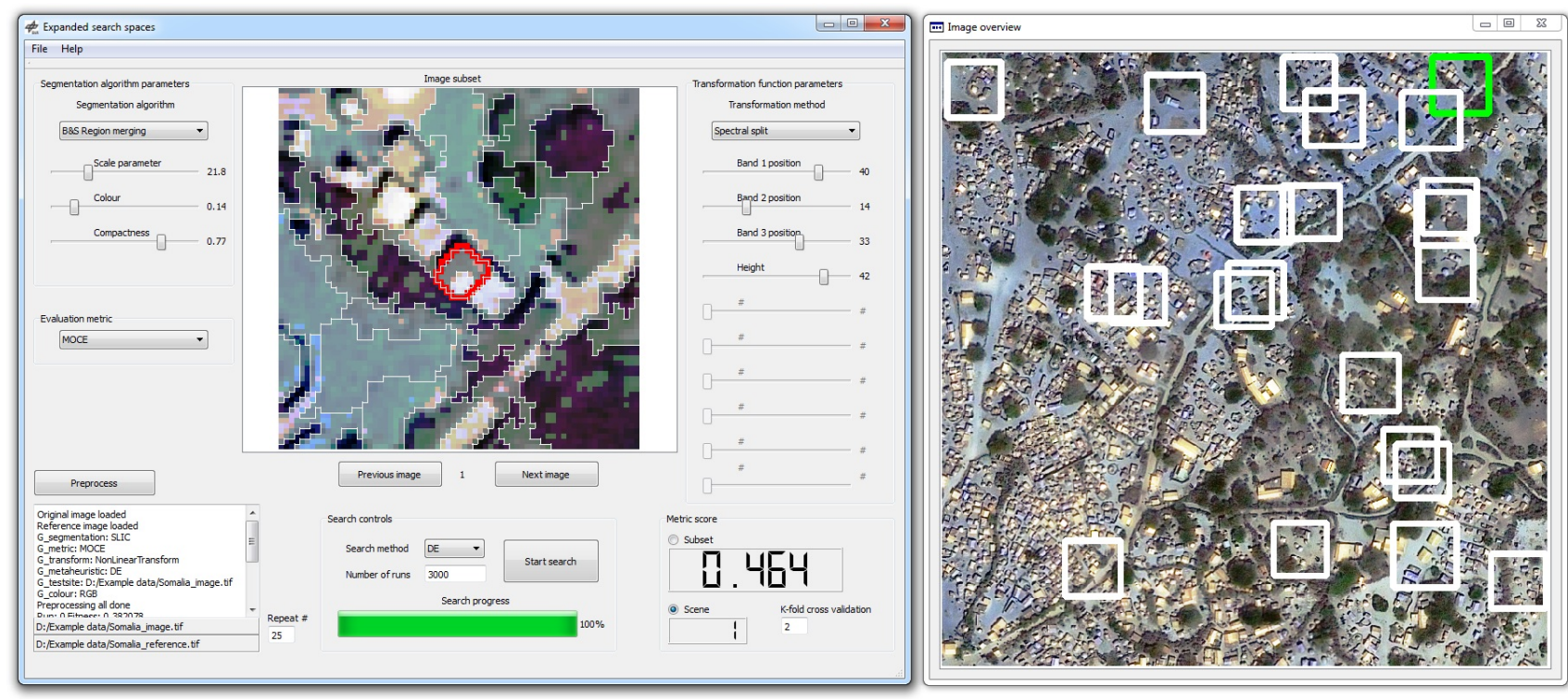

Figure 3.4. An example user interface for the search centric sample supervised segment generation approach described in this work.

In practice such a method could be implemented as a user driven software application or add-on, as illustrated in Figure 3.4. A user could be given the ability to digitize or select reference segments as well as control the search process and assess the results in a quantitative and qualitative manner. Intervention in the parameter tuning process of the segmentation algorithm and the data transformation function could be facilitated with user input controls, for example via interactive parameter sliders to visualize changes in segment results. Thus the method could function in a manual or automatic 
manner. Such a tool or method could be used independently or as part of a more encompassing image analysis process.

\subsubsection{Differential Evolution Metaheuristic}

Due to the potentially complex search landscapes (e.g., Figure 3.3) generated by the parameterized algorithms and evaluation metrics, stochastic population-based search methods are commonly employed in sample supervised segment generation approaches [14,26,30]. Such search methods do not use any derivative information (gradients) from the search landscape to direct the search. These methods have multiple agents or individuals traversing the search landscape, with information exchange among the agents and individual behavior controlling the convergence and exploration characteristics of aforesaid methods.

The Differential Evolution (DE) metaheuristic is used here, specifically the " $\mathrm{DE} / \mathrm{rand} / 1 / \mathrm{bin}$ " variant detailed in [53]. DE generalizes well over a range of problems, is intuitive and relatively easy to implement. For experimental conformity the population size is kept constant at 30 agents (random starting positions), although the search landscape dimensionalities range from two to thirteen. See [53] for details on the formulation of DE. The amplification factor of the difference agent (F) is set to 0.75 and the crossover constant (CR) to 0.3. DE was chosen and tuned based on preliminary experimentation [18] comparing it with other simple metaheuristics (particle swarm optimization, modified cuckoo search) for typical problem types addressed here, also corroborated with findings elsewhere [30,54].

\subsubsection{Data Transformation Functions}

In image processing simple data transformation or mapping functions are typically employed for image enhancement, implying an image is changed for either easier interpretation or for easier/more accurate further processing. Functions are typically point- or neighborhood-based, taking as input pixel values and giving as output new values. Point-based functions modify values taking as input only data from the given pixel (e.g., contrast stretch). Neighborhood-based functions also use data from surrounding pixels to modify a pixel (e.g., smoothing and edge-detection filters). Transformation functions may have controlling parameters influencing the output. In this work four transformation functions possessing controlling parameters are tested. All input imagery is assumed to consist of three bands.

Figure 3.5 illustrates an unmodified subset of a satellite image depicting small structures (Figure 3.5a), along with four transformation functions (Figure 3.5b-e) investigated in this work. Controlling parameters were given random values in generating the figures. The transformation functions vary in terms of observing local and neighborhood properties, observing multiple bands, algorithm flexibility and number of parameters. The four transformation functions are briefly described.

\subsubsection{Spectral Split}

Spectral Split [52] is a simple $n+1$ parameter transformation function where $n$ is the number of bands in the image. The transformation function changes the values of pixels around a specified, parameterized value called position. For each band $(n)$ of the input image, there is a corresponding 
position parameter. Another parameter, called height, controls the magnitude of the change around the position parameter(s). Pixels outside the range height compared to position are unaffected by the transformation. Spectral Split can be written as:

$$
f\left(x_{n}\right)=\left\{\begin{array}{c}
x_{n}: \operatorname{abs}\left(x_{n}-\text { position }_{n}\right) \geq \text { height } \\
x_{n}+\left(x_{n}-\text { position }_{n}\right)-\operatorname{sign}\left(x_{n}-\text { position }_{n}\right) \times \text { height }
\end{array}\right.
$$

where the sign function returns either -1 or 1 depending on the sign of the input. The $a b s$ function returns the absolute value of the input. Spectral Split can create areas of spectral continuity and also strong discontinuities (see Figure 3.5b). The low number of controlling parameters (four when concerned with a three band image) of the function may limit its ability to be effectively tailored for a specific problem. A simple modification to the function could see a separate height parameter for each input band.

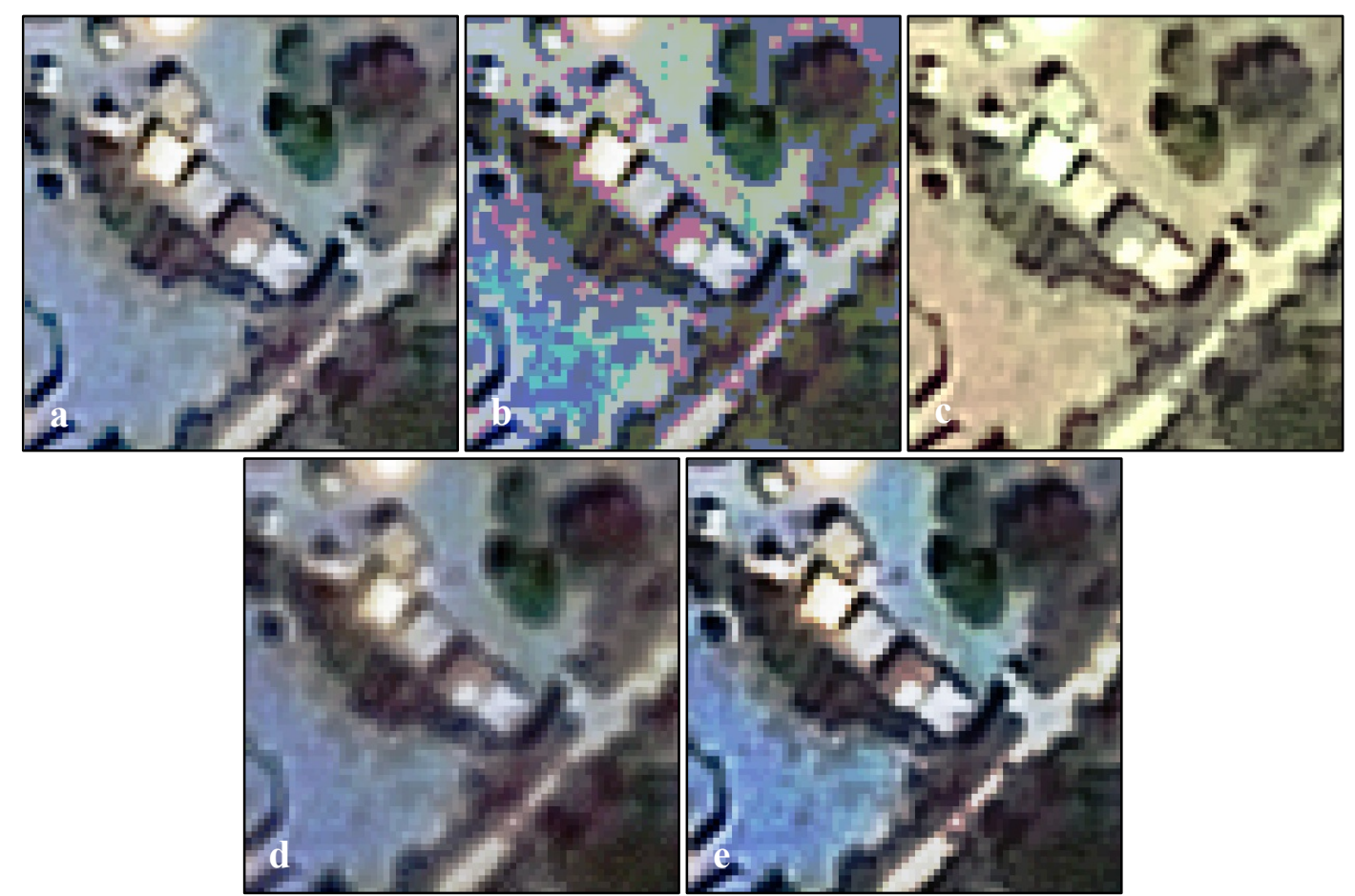

Figure 3.5. Output imagery from four data transformation functions tested in this work as part of a combined search space: (a) No Transformation, (b) Spectral Split, (c) Transformation Matrix, (d) Genetic Contrast, (e) Genetic Transform.

\subsubsection{Transformation Matrix}

A transformation matrix is tested here as a data transformation function (entitled Transformation Matrix), commonly used for color space transformations. Pixels of a given coordinate of an image stack can be represented as a point matrix. Multiplying the point matrix with a transformation matrix gives as output a new transformed point matrix. In the case of three image bands, such a transformation matrix consists of nine variables, modelled here as parameters for an image transformation (Equation (2)). Such a transformation allows certain bands to carry more weight, or highlight certain synergies of the data (see Figure $3.5 \mathrm{c}$ ). The range of the parameters is set to $[-0.2,1]$. As opposed to the other transforms described here, which only consider pixel values, spatial aspects 
and parameter values, the Transformation Matrix allows for a change of pixel values based on parameters and values from other channels.

$$
\left[\begin{array}{l}
\mathrm{n} 1 \\
\mathrm{n} 2 \\
\mathrm{n} 3
\end{array}\right]=\left[\begin{array}{lll}
a & b & c \\
d & e & f \\
g & h & i
\end{array}\right]\left[\begin{array}{l}
b 1 \\
b 2 \\
b 3
\end{array}\right]
$$

\subsubsection{Genetic Contrast}

Some works also propose to consider image contrast enhancement as a parameterized optimization problem, with a variant considering local spectral distributions proposed in $[48,55]$. A real valued encoding variant [55] of this image enhancement method is tested as a low-level transform, entitled Genetic Contrast for convenience. Genetic Contrast changes a pixel's value based on global image spectral characteristics, local characteristics (neighborhood standard deviation and mean values) and four controlling parameters (see Figure 3.5d). Genetic Contrast is written as:

$$
f(x)=\frac{k \times G}{\sigma(x)+b}(x-c \times m(x))+m(x)^{a}
$$

With $G$ denoting the image global mean value, $\sigma(x)$ the standard deviation around pixel $x$ and $m(x)$ the mean around pixel $x$. Controlling parameters are denoted with $a, b, c$, and $k$, with ranges $[0,1.5]$ for $a,[0, G / 2]$ for $b,[0,1]$ for $c$, and $[0.5,1.5]$ for $k$.

\subsubsection{Genetic Transform}

General approaches have also been put forth that consider low-level image processing as an optimization problem, with some of the early works focussing on basic image enhancement $[49,56]$. Such an approach is investigated here as a potential low-level transform forming part of the combined low- and mid-level image processing search landscape. A ten parameter transformation function $[49,56]$, for convenience called Genetic Transform, is tested. Genetic Transform consists of four parameter-controlled non-linear transformation functions, with either one or two controlling parameters. These four functions are weighted (convex combination) by four additional parameters to form a singular function. This allows for flexibility in the prominence of the different transforms, in addition to flexibility in the actual structures of the mappings. All bands in an image are handled with the same functions (parameters). Input values are scaled to the range $[0,1]$. The four transforms are written as Equations (4-7).

$$
\begin{gathered}
f 1(x)=\frac{\log \left(1+e^{p 1-1} \times x\right)}{p 1} \\
f 2(x)=\frac{(1+p 2)^{x}-1}{p 2} \\
f 3(x)=\frac{1}{1+\left(\frac{(1-x)}{p 3}\right)^{p 4}} \\
f 4(x)=\frac{1}{\left(\frac{1}{x}+p 6-1\right)^{p 5}}
\end{gathered}
$$


Figure 3.6 illustrates example mappings of the four functions with arbitrarily chosen parameter values. The combined function is written as:

$$
f(x)=p 7 \times f 1(x)+p 8 \times f 2(x)+p 9 \times f 3(x)+p 10 \times f 4(x)
$$

Similar to Spectral Split, Genetic Transform is a point-based function that could lead to stronger spectral discontinuities or continuities due to the non-linear rescaling of the data (Figure 3.5e). In contrast, it has more controlling parameters, increasing search landscape dimensionality, but is theoretically more flexible and may, thus, fit better to a given problem.

\subsubsection{Image Segmentation Algorithms}

Two image segmentation algorithms are tested within the approach described, a fast clustering algorithm and a variant of region-merging segmentation.

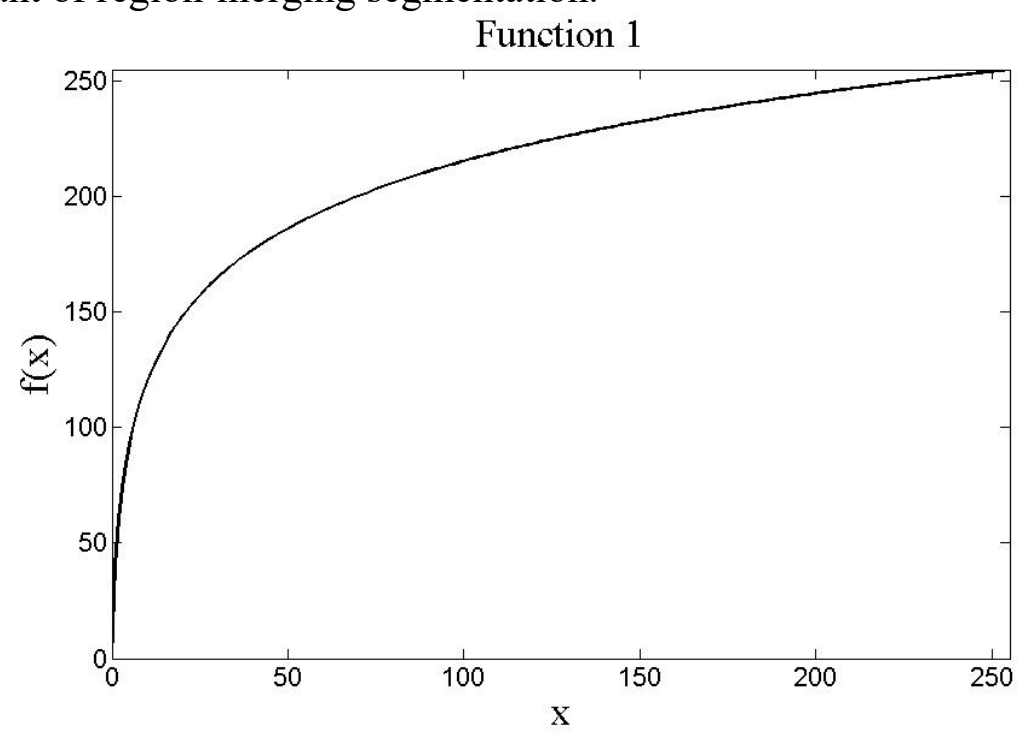

Function 2

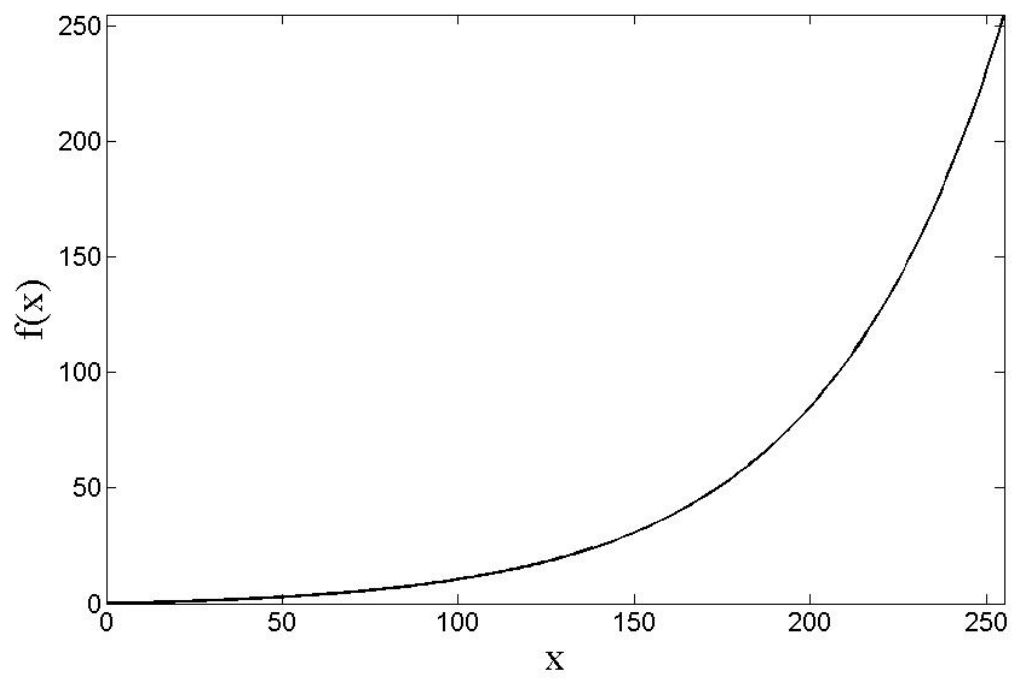

Figure 3.6. Cont. 
Function 3

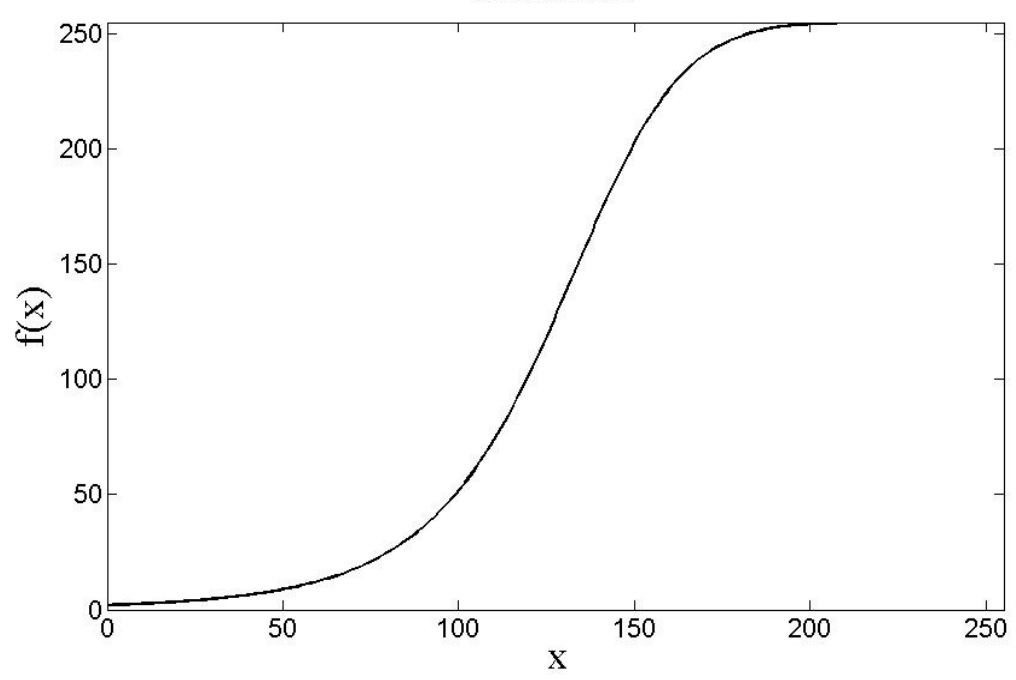

Function 4

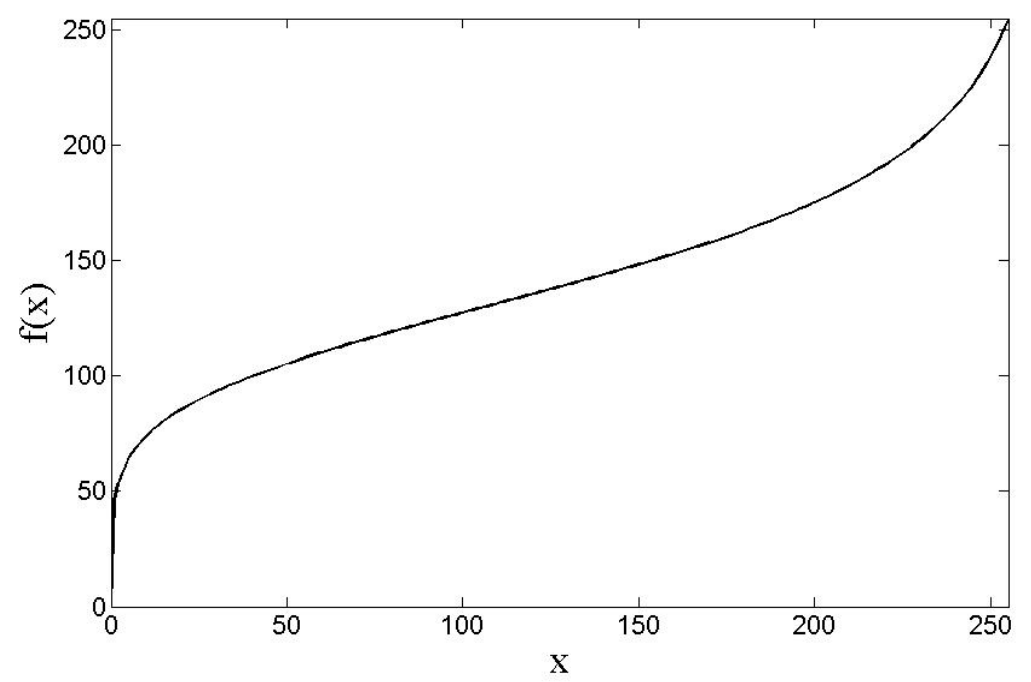

Figure 3.6. Four parameterized transformation functions, weighted to constitute the Genetic Transform function.

\subsubsection{Simple Linear Iterative Clustering Algorithm}

Simple Linear Iterative Clustering (SLIC) [57] is considered a superpixel algorithm-which attempts to group an image into larger spectral atomic units as opposed to the original pixel level. The algorithm is intended for use on natural color imagery. Semantic segmentation is typically not attempted with such algorithms, but results may be used in further segmentation processes. The SLIC algorithm clusters (segments) pixels in a five dimensional space using region constrained k-means clustering. The space consists of the two dimensions of the image ( $\mathrm{x}$ and $\mathrm{y}$ dimensions) and three input bands; which are assumed to be in the red, green, blue color space. The SLIC algorithm converts the RGB color space to the CIELAB color space before further processing (not done in this work). SLIC is used here with some liberty in the context of semantic image segmentation due to the computational efficiency of the algorithm, allowing for near real time manual segmentation algorithm parameter tuning, and middling segmentation results on problems addressed in this work.

\subsubsection{Multiresolution Segmentation}


The basic Multiresolution Segmentation (MS) algorithm [10] is a three parameter variant of region-merging segmentation. The scale parameter of the MS algorithm, which incorporates notions of the spectral degree of fitting before and after a virtual segment merge and object size, controls the relative sizes of segments. Two other parameters namely shape/color and compactness/smoothness controls the weight of two geometry homogeneity criteria (compactness and smoothness [10]) against each other (compactness/smoothness) and against the scale parameter (shape/color). Other geometric homogeneity criteria may also be considered [29]. A variant not considering band weighting [10] is utilized.

\subsubsection{Spatial Metrics}

Four empirical discrepancy metrics are employed as fitness functions to direct the search process. These four fitness functions are utilized in the context of single-objective optimization and use notions of spatial overlap, as opposed to edge matching or empirical goodness metrics. Doing comparative experimentation with multiple fitness functions are advocated (four in our case), as quality are measured differently among the functions. Various metrics matching a single generated segment to a reference segment have been shown to be highly correlated [15], with a singular metric in the test bed considering over- and under segmentation more elaborately diverging from the correlation [15]. Thus, results are reported with a metric matching a single generated segment to that of a reference segment and three metrics allowing for multiple generated segments to be matched with a given reference segment (having internal differences). Metrics allowing for multiple generated segments to be matched with a single reference segment are more flexible to a wide range of problem instances, for example if some reference segments are routinely over-segmented or if, due to segmentation algorithm limitations, over-segmentation is common.

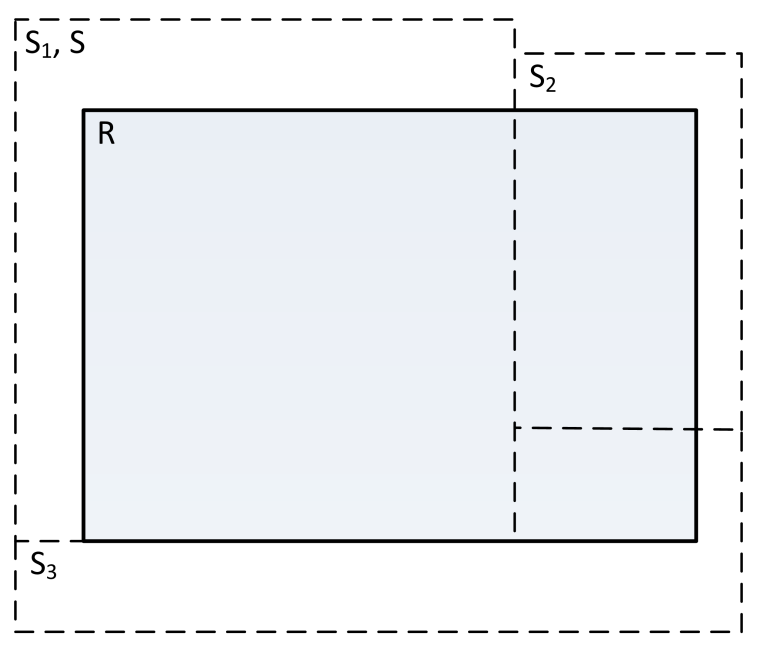

Figure 3.7. An abstract segmentation evaluation scenario with the reference segment denoted by $\mathrm{R}$, the generated segments by $\mathrm{Si}$ and the generated segment with the largest overlap with $\mathrm{R}$ as $\mathrm{S}$.

Figure 3.7 illustrates an abstract segmentation evaluation scenario with the reference segment denoted by $\mathrm{R}$, generated segments intersecting the reference segment by $\mathrm{S}_{\mathrm{i}}$, the generated segment with the largest overlap with the reference segment as $\mathrm{S}$ and the number of segments intersecting with $\mathrm{R}$ as $\mathrm{n}$. Results of such evaluations are averaged over multiple reference segments. The four utilized metrics 
are described using set theory notation. "I |" Denotes the cardinality (the number of pixels in the segment). The optimal value for all metrics described here is zero.

\subsubsection{Reference Bounded Segments Booster}

The Reference Bounded Segments Booster (RBSB) [25] metric measures the amount of mismatch between $\mathrm{R}$ and $\mathrm{S}$ against $\mathrm{R}$, with a range of $[0, \infty]$ and can be written as:

$$
R B S B=\frac{|R \cup S|-|R \cap S|}{|R|}
$$

\subsubsection{Larger Segments Booster}

A variant of the Larger Segments Booster (LSB) [31] metric is used here, written as:

$$
L S B=\frac{\left|R \cup S_{h}\right|-\left|R \cap S_{h}\right|+b}{|R|}
$$

where $S_{h}=S_{i} \cup S_{i+1} \cup \ldots \cup S_{n}$ and with each $S_{i}$ having at least half of its pixels in $R . b$ Denotes the number of pixels intersecting $R$.

\subsubsection{Partial and Directed Object-Level Consistency Error}

Inspired by the Object-level Consistency Error (OCE) [17], which is influenced by the Jaccard index [58], a partial and directed variant of OCE is defined, entitled PD_OCE, with a range of [0, 1] and written as:

$$
P D \_O C E=\sum_{i=1}^{n} \frac{\left|R \cap S_{i}\right|}{\left|R \cup S_{i}\right|} \times \frac{\left|S_{i}\right|}{\sum_{j=1}^{n}\left|S_{j}\right|}
$$

A specific $\mathrm{S}_{\mathrm{i}}$ covering only a fraction of $\mathrm{R}$ but with a very large component outside of $\mathrm{R}$ have a large influence on the metric score, which could be an erroneous quality notion in many problem instances.

\subsubsection{Reference Weighted Jaccard}

Also inspired by the Jaccard index, a new metric is proposed, the Reference Weighted Jaccard $(\mathrm{RWJ})$, with a range of $[0,1]$ and written as:

$$
R W J=\sum_{i=1}^{n} \frac{\left|R \cap S_{i}\right|}{\left|R \cup S_{i}\right|} \times \frac{\left|R \cap S_{i}\right|}{|R|}
$$

As opposed to the OCE/PD_OCE metric, the RWJ metric weights the summation of results against the contribution of $S_{i}$ to $R$.

\subsubsection{Examples of Data Transforms with Segments and Metric Scores}

Figure 3.8 illustrates several different combinations of data transformation function parameters (Spectral Split) and segmentation algorithm parameters (Multiresolution Segmentation) in terms of RWJ metric scores. The other parameters of the segmentation algorithm and transformation function were kept constant. Note the differences in metric scores when considering a MS scale parameter of 50. 


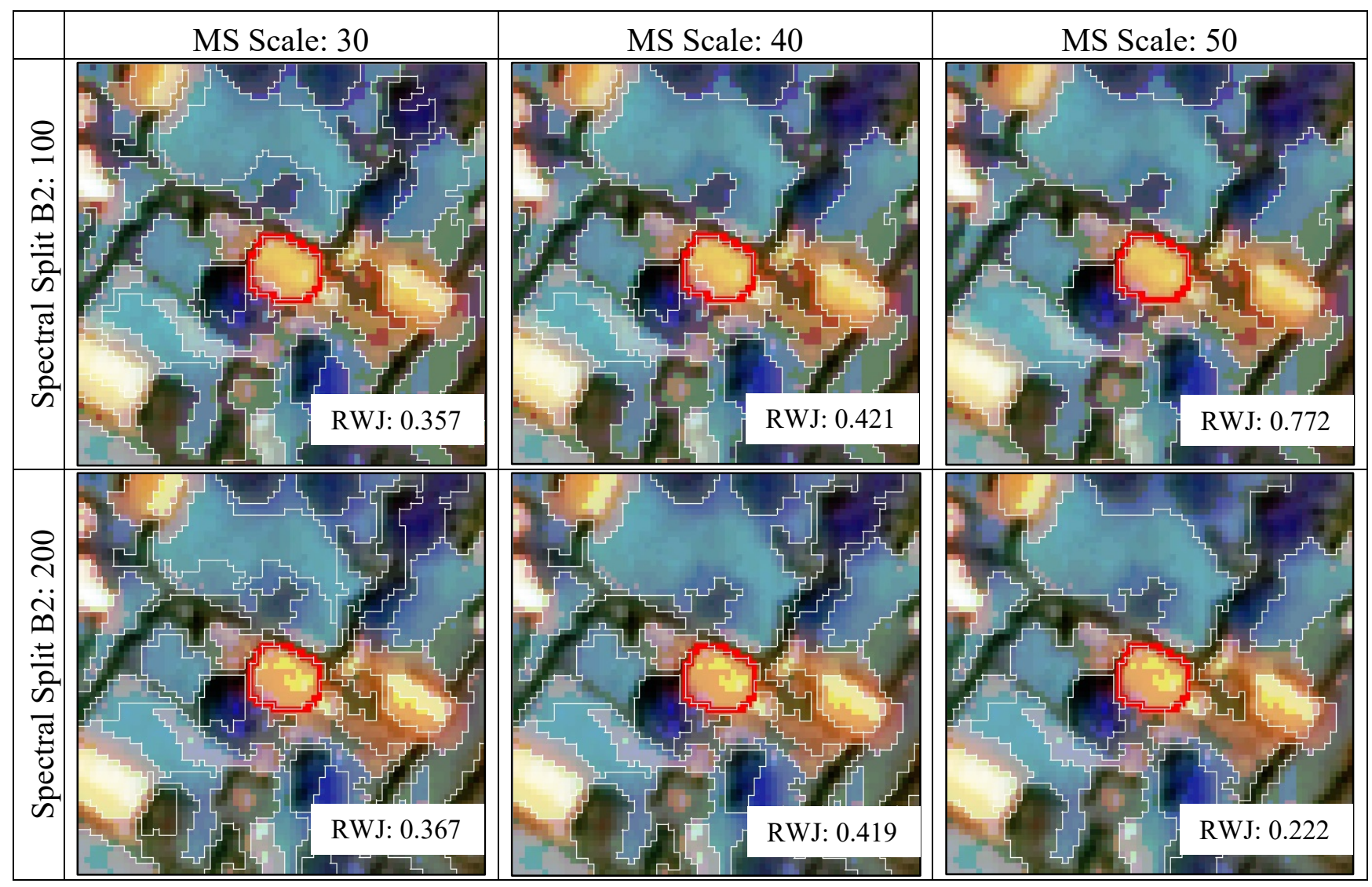

Figure 3.8. RWJ metric scores on a single object (house) of interest delineated by a bold red line. The Multiresolution Segmentation algorithm and Spectral Split transformation function were employed.

\subsection{Data}

Four example problems are addressed and tested with this approach. Fully pre-processed subsets of VHR optical imagery (Figure 3.9) were obtained, depicting a settlement (Jowhaar, Figure 3.9a), refugee camps (Hagadera, Bokolmanyo, Figure 3.9b,c) and a settlement surrounded by informal agriculture (Akonolinga, Figure 3.9d). The aims could be to obtain information for conducting urban planning (Figure 3.9a), structure counting and characterization (Figure 3.9b,c) and agricultural monitoring for land-use planning (Figure 3.9d). It is attempted to segment structures (Figure 3.9a-c) and fields (Figure 3.9d) using a single segmentation layer using the sample supervised segment generation approach described. Provided reference segments are enclosed by white bounding boxes. The Bokolmanyo site (Figure 3.9c) pose an easy segmentation problem, with the other three problems proving to be more difficult to segment accurately using only a single segment layer. In practice subsequent processes could refine results, especially on the more difficult problems. All imagery consists of three input bands, have 8-bit quantization and are pansharped. Table 3.1 lists some image metadata, including the number of utilized reference objects or segments for each image. 

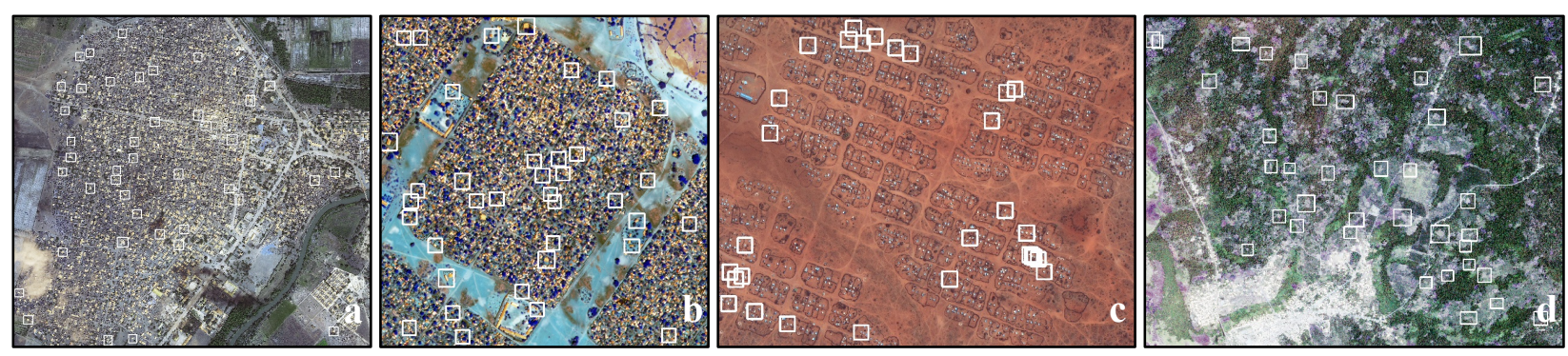

Figure 3.9. The four datasets used in this study, entitled (a) Jowhaar, (b) Hagadera, (c) Bokolmanyo, (d) Akonolinga. Dataset details are given in Table 3.1.

Table 3.1. Dataset metadata, including the number of utilized reference segments.

\begin{tabular}{|c|c|c|c|c|c|c|}
\hline Test Site & $\begin{array}{c}\text { Target } \\
\text { Elements }\end{array}$ & Sensor & $\begin{array}{c}\text { Spatial } \\
\text { Resolution }\end{array}$ & $\begin{array}{l}\text { Reference } \\
\text { Segments }\end{array}$ & Channels & $\begin{array}{c}\text { Date } \\
\text { Captured }\end{array}$ \\
\hline Jowhaar $^{1}$ & Structures & GeoEye-1 & $0.5 \mathrm{~m}$ & 40 & $1,2,3$ & $2011 / 02 / 26$ \\
\hline Hagadera $^{2}$ & Structures & WorldView-2 & $0.5 \mathrm{~m}$ & 38 & $4,6,3$ & $2010 / 10 / 07$ \\
\hline Bokolmanyo ${ }^{1}$ & Tents & GeoEye-1 & $0.5 \mathrm{~m}$ & 28 & $1,2,3$ & $2011 / 08 / 24$ \\
\hline Akonolinga $^{3}$ & Fields & QuickBird & $2.4 \mathrm{~m}$ & 35 & $1,2,3$ & $2008 / 03 / 06$ \\
\hline
\end{tabular}

\subsection{Methodology}

The method described here is quantitatively compared in terms of segmentation accuracies with a general sample supervised segment generation approach not performing any data transformations (detailed in Section 3.4.1). Several search methods are investigated with such an approach, to validate the usefulness of more complex variants (Section 3.4.2). In addition to the segmentation evaluation, some convergence profiling is done on variants of the method. Finally the presences of parameter interdependencies are investigated to justify the creation of enlarged search spaces (Section 3.4.3).

\subsubsection{Data Transformation Functions for Expanded Search Spaces}

The general usefulness of transformation function expanded search spaces is investigated. The four sites and reference segments detailed in Section 3.3 (Figure 3.9) is used with the sample supervised segment generation approach detailed in Section 3.2. For each site, the method is executed using the four transforms described in Section 3.2.3 and using the MS and SLIC segmentation algorithms. In addition to using the transforms, the method is also run using no data transformation function. Evaluation is conducted using the four different evaluation metrics described in Section 3.2.5. In total, 40 different experimental setups or methodological variants are tested per site, allowing for evaluating the usefulness of such an approach in general, but also giving indicators of the usefulness of specific transforms and segmentation algorithms.

The utilized DE metaheuristic is given 3000 search iterations (30 agents) to traverse the search space in all experimental instances, thus, not giving enlarged search spaces more computing time or evaluations. All experiments are repeated 25 times to generate a measure of specificity and also to 
allow for testing the statistical significance among method variants, amounting to \pm 250 million segmentation evaluations. Two-fold (hold-out) cross-validation is performed in all experimental runs to prevent overfitting of the parameter sets to the given reference segments.

\subsubsection{Metaheuristic Evaluation and Convergence Behavior Profiling}

The appropriateness of using a population based metaheuristic on the search spaces created by combining simple transformation function parameters and segmentation algorithm parameters is investigated. For two of the test sites, the proposed method is run using four different transformation function strategies. The DE metaheuristic is compared for such applications (search landscapes) with simple to implement search strategies, namely a Hill Climber (HC) and Random Search (RND). For each test site, using the different transformation function strategies, the three search methods are compared via fitness trace characteristics and resultant segment quality after 3000 search iterations. Fitness traces/plots are averaged over 25 runs.

The convergence behavior of the method operating under no data transformation and data transformation conditions is also investigated. Four of the transformation strategies are compared. Due to the computationally expensive nature of such an approach, a search process should typically be terminated as soon as possible. It is investigated how larger search space variants compare with simpler, lower dimensional, variants in terms of convergence behavior, for example to determine if simpler variants have some early accuracy advantage over complex variants.

\subsubsection{Parameter Domain Interdependencies}

As with classification, search processes can suffer from the curse of dimensionality. Problems could be decomposed into smaller separate problems for more efficient processing if there exist no interdependencies [50,51] among the processes or parameter domains. Some processes could add value to a solution without, or minimally, interacting with other processes. In the context of GEOBIA, examples could be post hierarchical segmentation segment merging procedures observing attribute criteria, contextual classification not sensitive to segment characteristics (segmentation algorithm parameters) or a form of spectral transformation and segmentation (e.g., mathematical morphology) resulting in slightly different/better segments, as opposed to not performing the spectral transformation, but having identical segmentation algorithm parameters.

Tests are conducted to validate the existence of interdependencies between the data transformation function parameters and segmentation algorithm parameters used in this study, which can be strongly anticipated in this context. The sample supervised method described here is run on a test site using the two segmentation algorithms, four metrics and four transforms in selected combinations. The optimal segmentation algorithm parameter values generated by the variants of the method are recorded (25 runs) and contrasted, specifically the differences when using different transforms versus using no transformation function. Differing optimal values gives an indication of interdependencies.

Furthermore a simple statistical variable interdependency test is performed between parameters from the segmentation algorithm and data transformation function domains, as detailed in [50]. A variable or parameter $x_{i}$ is affected by $x_{j}$ if, given a parameter set $a=\left(\ldots, x_{i}, \ldots, x_{j}, \ldots\right)$ and $b=\left(\ldots, x_{i}^{\prime}, \ldots, x_{j}, \ldots\right)$ and $f(a) \leq f(b)$ (fitness evaluation), with a change of $x_{j}$ to $x_{j}^{\prime}$ in sets $a$ and $b$ to create $a^{\prime}$ and $b^{\prime}$, the equation $f\left(a^{\prime}\right)>f\left(b^{\prime}\right)$ holds. For one site, using the MS segmentation algorithm 
and Spectral Split transformation function, an exhaustive parameter dependency test based on 100 randomly initiated values for the described evaluation is performed. Any value above 0 indicates variable interaction, although higher values suggest more regular interaction.

\subsection{Results and Discussion}

\subsubsection{Data Transformation Functions for Expanded Search Spaces}

Tables 3.2-5 list the results of the experimental runs on the four test sites. The values indicate the best fitness achieved, averaged over 25 runs. The standard deviations are also given. Different data transformation functions may be contrasted within each metric category (horizontal grouping). Lower values indicate better results. Results from different metrics cannot be compared (vertical grouping), although the results from the two segmentation algorithms within each metric category may be contrasted. The shaded cells delineate results obtained using variants of the method employing data transformations that resulted in worse quality scores compared to the variant of the method using no data transformations. Results that are not statistically significantly different from the no transformation variant according to the student's t-test with a 95\% confidence interval are also delineated with shaded cells.

Table 3.2. Jowhaar site results.

\begin{tabular}{ccccccc}
\hline Jowhaar & & No Transform & Spectral Split & $\begin{array}{c}\text { Transformation } \\
\text { Matrix }\end{array}$ & $\begin{array}{c}\text { Genetic } \\
\text { Transform }\end{array}$ & $\begin{array}{c}\text { Genetic } \\
\text { Contrast }\end{array}$ \\
\hline \multirow{2}{*}{ RBSB } & SLIC & $0.49 \pm 0.03$ & $0.43 \pm 0.06$ & $0.36 \pm 0.03$ & $0.39 \pm 0.06$ & $0.47 \pm 0.04$ \\
& MS & $0.31 \pm 0.04$ & $0.31 \pm 0.05$ & $0.25 \pm 0.07$ & $0.32 \pm 0.04$ & $0.35 \pm 0.06$ \\
\multirow{2}{*}{ PD_OCE } & SLIC & $0.82 \pm 0.01$ & $0.80 \pm 0.01$ & $0.69 \pm 0.02$ & $0.72 \pm 0.02$ & $0.85 \pm 0.01$ \\
& MS & $0.78 \pm 0.01$ & $0.73 \pm 0.02$ & $0.57 \pm 0.03$ & $0.61 \pm 0.03$ & $0.80 \pm 0.01$ \\
RWJ & SLIC & $0.45 \pm 0.01$ & $0.43 \pm 0.03$ & $0.34 \pm 0.02$ & $0.41 \pm 0.03$ & $0.49 \pm 0.02$ \\
& MS & $0.30 \pm 0.02$ & $0.30 \pm 0.02$ & $0.22 \pm 0.02$ & $0.32 \pm 0.02$ & $0.39 \pm 0.02$ \\
& LSB & $0.45 \pm 0.00$ & $0.43 \pm 0.01$ & $0.30 \pm 0.03$ & $0.41 \pm 0.01$ & $0.48 \pm 0.02$ \\
& MS & $0.33 \pm 0.02$ & $0.33 \pm 0.03$ & $0.26 \pm 0.02$ & $0.31 \pm 0.02$ & $0.37 \pm 0.02$ \\
\hline
\end{tabular}

Table 3.3. Hagadera site results.

\begin{tabular}{ccccccc}
\hline Hagadera & & No Transform & Spectral Split & $\begin{array}{c}\text { Transformation } \\
\text { Matrix }\end{array}$ & $\begin{array}{c}\text { Genetic } \\
\text { Transform }\end{array}$ & $\begin{array}{c}\text { Genetic } \\
\text { Contrast }\end{array}$ \\
\hline \multirow{2}{*}{ RBSB } & SLIC & $0.47 \pm 0.00$ & $0.44 \pm 0.03$ & $0.31 \pm 0.03$ & $0.49 \pm 0.04$ & $0.52 \pm 0.07$ \\
& MS & $0.60 \pm 0.07$ & $0.53 \pm 0.09$ & $0.39 \pm 0.09$ & $0.51 \pm 0.12$ & $0.70 \pm 0.15$ \\
PD_OCE & SLIC & $0.80 \pm 0.00$ & $0.78 \pm 0.01$ & $0.64 \pm 0.04$ & $0.77 \pm 0.01$ & $0.78 \pm 0.01$ \\
& MS & $0.78 \pm 0.02$ & $0.74 \pm 0.02$ & $0.62 \pm 0.02$ & $0.74 \pm 0.02$ & $0.80 \pm 0.02$ \\
RWJ & SLIC & $0.47 \pm 0.02$ & $0.49 \pm 0.02$ & $0.37 \pm 0.01$ & $0.45 \pm 0.01$ & $0.51 \pm 0.01$ \\
& MS & $0.49 \pm 0.02$ & $0.45 \pm 0.03$ & $0.39 \pm 0.03$ & $0.46 \pm 0.03$ & $0.51 \pm 0.03$ \\
LSB & SLIC & $0.58 \pm 0.02$ & $0.58 \pm 0.02$ & $0.41 \pm 0.02$ & $0.56 \pm 0.03$ & $0.65 \pm 0.02$ \\
& MS & $0.58 \pm 0.03$ & $0.57 \pm 0.04$ & $0.49 \pm 0.03$ & $0.56 \pm 0.03$ & $0.65 \pm 0.04$ \\
\hline
\end{tabular}

Examining Tables 3.2-5 it is evident that simple expanded search spaces (under identical search conditions) can assist in generating better quality segments. The magnitude of the improvement depends on the definition of quality (metric), the characteristics of the features of interest in the image 
as well as the specific data transformation function employed. Notwithstanding, under certain metric and transformation function conditions, consistently worse results are obtained. The Genetic Contrast transformation function in particular does not add value to most problem instances investigated here. This could be due to the smoothing/distorting effect of the function (Figure 3.5d). In addition, the Spectral Split function only improves results fractionally, if at all.

Table 3.4. Bokolmanyo site results.

\begin{tabular}{|c|c|c|c|c|c|c|}
\hline Bokolmanyo & & No Transform & Spectral Split & $\begin{array}{c}\text { Transformation } \\
\text { Matrix }\end{array}$ & $\begin{array}{c}\text { Genetic } \\
\text { Transform } \\
\end{array}$ & $\begin{array}{c}\text { Genetic } \\
\text { Contrast }\end{array}$ \\
\hline \multirow[t]{2}{*}{ RBSB } & SLIC & $0.23 \pm 0.00$ & $0.28 \pm 0.09$ & $0.20 \pm 0.04$ & $0.19 \pm 0.02$ & $0.28 \pm 0.05$ \\
\hline & MS & $0.22 \pm 0.07$ & $0.21 \pm 0.05$ & $0.18 \pm 0.04$ & $0.15 \pm 0.04$ & $0.34 \pm 0.12$ \\
\hline \multirow[t]{2}{*}{ PD_OCE } & SLIC & $0.78 \pm 0.00$ & $0.64 \pm 0.04$ & $0.50 \pm 0.03$ & $0.46 \pm 0.03$ & $0.75 \pm 0.03$ \\
\hline & MS & $0.75 \pm 0.02$ & $0.56 \pm 0.04$ & $0.39 \pm 0.03$ & $0.36 \pm 0.03$ & $0.76 \pm 0.03$ \\
\hline \multirow[t]{2}{*}{ RWJ } & SLIC & $0.33 \pm 0.01$ & $0.32 \pm 0.04$ & $0.23 \pm 0.02$ & $0.25 \pm 0.02$ & $0.42 \pm 0.02$ \\
\hline & MS & $0.29 \pm 0.01$ & $0.26 \pm 0.02$ & $0.20 \pm 0.02$ & $0.22 \pm 0.02$ & $0.39 \pm 0.03$ \\
\hline \multirow[t]{2}{*}{ LSB } & SLIC & $0.60 \pm 0.01$ & $0.58 \pm 0.04$ & $0.49 \pm 0.03$ & $0.50 \pm 0.03$ & $0.68 \pm 0.02$ \\
\hline & MS & $0.48 \pm 0.01$ & $0.46 \pm 0.03$ & $0.40 \pm 0.04$ & $0.39 \pm 0.02$ & $0.64 \pm 0.05$ \\
\hline
\end{tabular}

Table 3.5. Akonolinga site results.

\begin{tabular}{ccccccc}
\hline \multirow{2}{*}{ Akonolinga } & & No Transform & Spectral Split & $\begin{array}{c}\text { Transformation } \\
\text { Matrix }\end{array}$ & $\begin{array}{c}\text { Genetic } \\
\text { Transform }\end{array}$ & $\begin{array}{c}\text { Genetic } \\
\text { Contrast }\end{array}$ \\
\hline \multirow{2}{*}{ RBSB } & SLIC & $0.61 \pm 0.00$ & $0.69 \pm 0.14$ & $0.62 \pm 0.06$ & $0.54 \pm 0.06$ & $0.58 \pm 0.02$ \\
& MS & $0.36 \pm 0.05$ & $0.38 \pm 0.07$ & $0.38 \pm 0.09$ & $0.38 \pm 0.16$ & $0.45 \pm 0.14$ \\
PD_OCE & SLIC & $0.82 \pm 0.00$ & $0.79 \pm 0.01$ & $0.78 \pm 0.02$ & $0.76 \pm 0.02$ & $0.84 \pm 0.01$ \\
& MS & $0.79 \pm 0.01$ & $0.77 \pm 0.01$ & $0.76 \pm 0.02$ & $0.76 \pm 0.02$ & $0.80 \pm 0.01$ \\
RWJ & SLIC & $0.46 \pm 0.01$ & $0.47 \pm 0.02$ & $0.44 \pm 0.03$ & $0.40 \pm 0.02$ & $0.41 \pm 0.01$ \\
& MS & $0.32 \pm 0.02$ & $0.33 \pm 0.02$ & $0.34 \pm 0.03$ & $0.32 \pm 0.02$ & $0.35 \pm 0.03$ \\
\hline \multirow{2}{*}{ LSB } & SLIC & $0.43 \pm 0.00$ & $0.44 \pm 0.02$ & $0.33 \pm 0.01$ & $0.31 \pm 0.01$ & $0.37 \pm 0.01$ \\
& MS & $0.33 \pm 0.02$ & $0.33 \pm 0.02$ & $0.32 \pm 0.02$ & $0.32 \pm 0.01$ & $0.34 \pm 0.02$ \\
\hline
\end{tabular}

Adding the Transformation Matrix and Genetic Transform functions on the other hand almost always results in improved segmentation accuracies, with varying magnitudes. Figure 3.10 highlights RWJ metric scores using SLIC as the segmentation algorithm. Note the performances of the Transformation Matrix and Genetic Transform functions. Under a few metric and image conditions (Tables 3.2-5), improvements in excess of a decrease of 0.20 in metric values are noted with these two transforms. In the eight instances where these functions did not improve results, the results were not worse by more than a 0.02 change in metric values (minor) and only requiring neglectable additional computing for performing the data transformation.

The behavior of the RBSB metric on the Akonolinga site using the SLIC segmentation algorithm could be due to the fact that the given range of the scale parameter in SLIC was not sufficiently large to segment some of the relatively large fields in this scene. RBSB matches the largest overlapping generated segment with the reference segment, potentially creating noise/discontinuities in these instances and thus causing difficulty for the search algorithm (30 randomly initialized agents). 


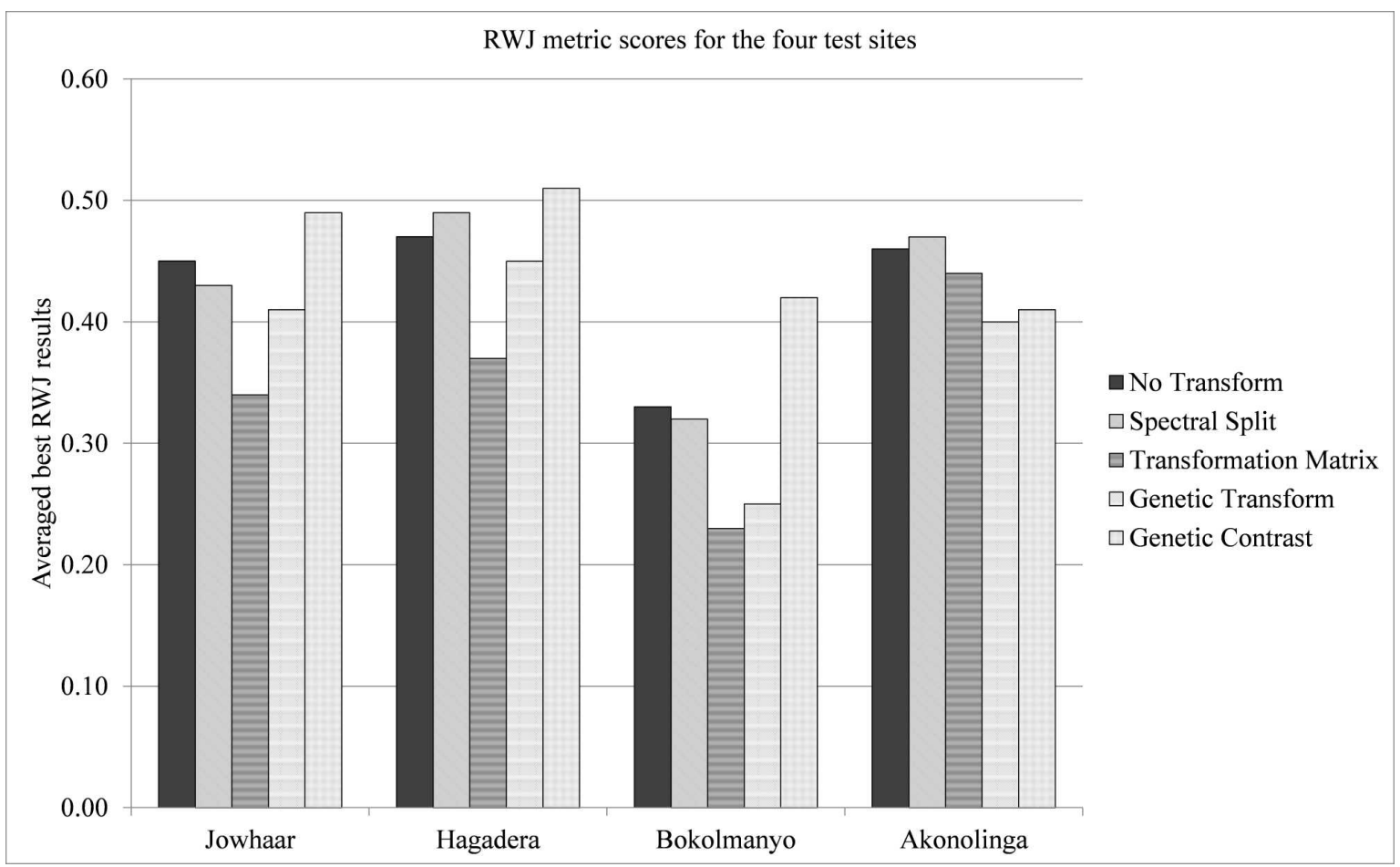

Figure 3.10. Averaged RWJ metric scores (lower is better) for SLIC segmentation on the four sites using the five different transformation function strategies.

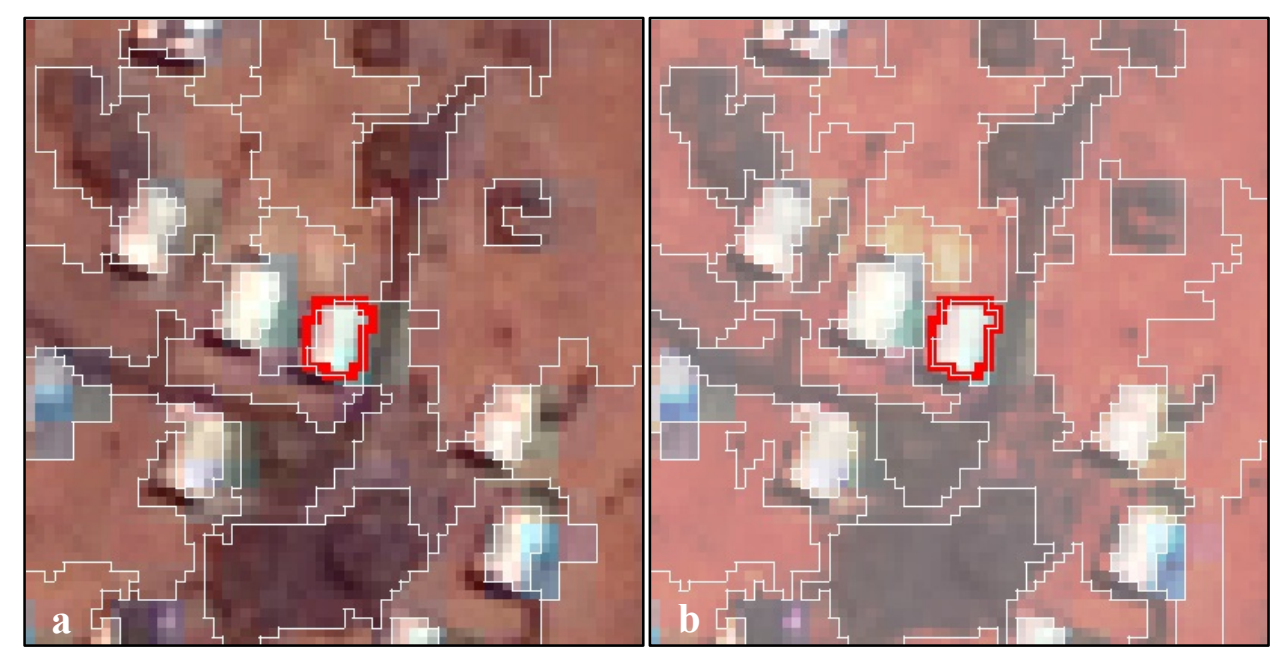

Figure 3.11. Example of best segmentation results achieved on (a) original image and (b) an image transformed with the Genetic Transform function for the Bokolmanyo site using the MS segmentation algorithm and RWJ metric.

Figures 3.11 and 3.12 highlight the best segmentations achieved with different transformation functions compared to the variant of the method not performing transformations. Note the more accurate boundaries on the reference segments (outlined in red) as well as better fits on structures not delineated due to averaging results and performing cross-validation to improve generalizability. Additional post-segmentation processing steps could improve results. In addition, note that image transformation is done solely for the purpose of generating better segments. Subsequent processes/methods should run on the original image data (e.g., Figure 3.11a), but using the segments generated on the transformed space (e.g., Figure 3.11b). 

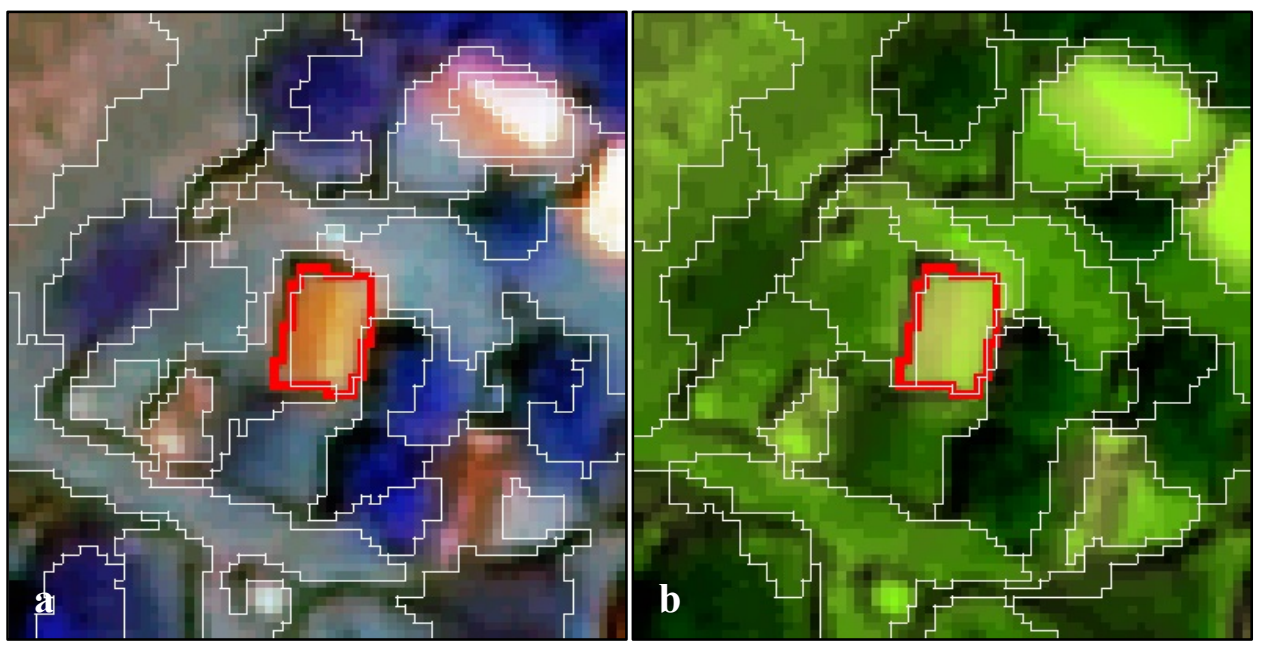

Figure 3.12. Example of best segmentation results achieved on (a) the original image and (b) an image transformed with the Transformation Matrix for the Hagadera site using the MS segmentation algorithm and RBSB metric.

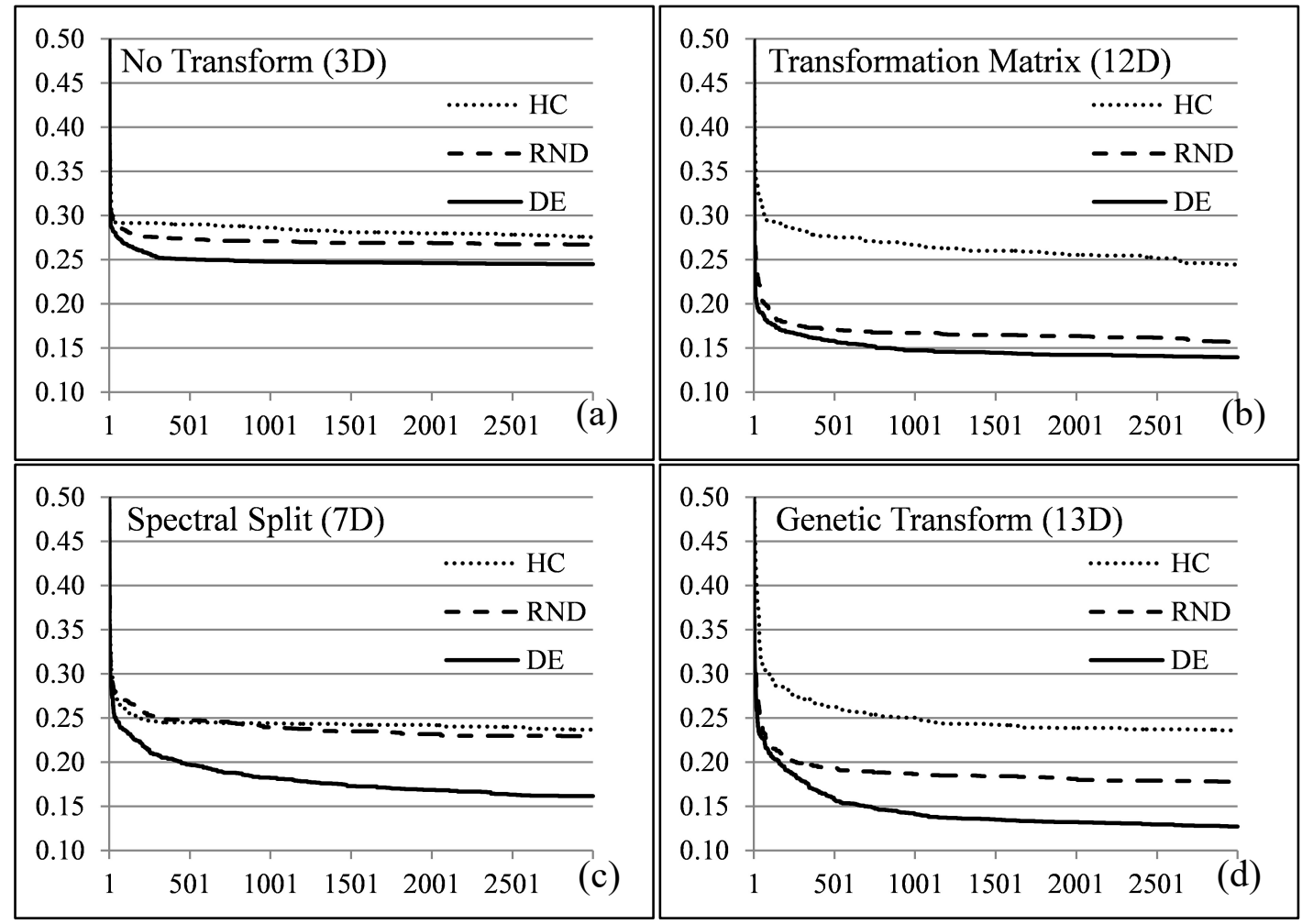

Figure 3.13. Fitness traces for three search methods on the Bokolmanyo site (MS segmentation) using the (a) No Transform, (b) Transformation Matrix, (c) Spectral Split and (d) Genetic Transform transformation strategies. The x-axes delineate search iterations. The y-axes delineate RWJ evaluation scores.

\subsubsection{Metaheuristic Evaluation and Convergence Behavior Profiling}

Figures 3.13 and 3.14 illustrate the fitness traces of the DE, HC and RND search methods averaged over 25 runs for the elements of interest (averaged) in the Bokolmanyo and Jowhaar sites. Each sub-graph illustrates results using a different image transformation strategy. The search landscape 
dimensionalities of the different method variants are given in brackets. Final metric scores may differ from results presented in Section 3.5.1 due to cross-validation, which is not possible here for a fitness trace.

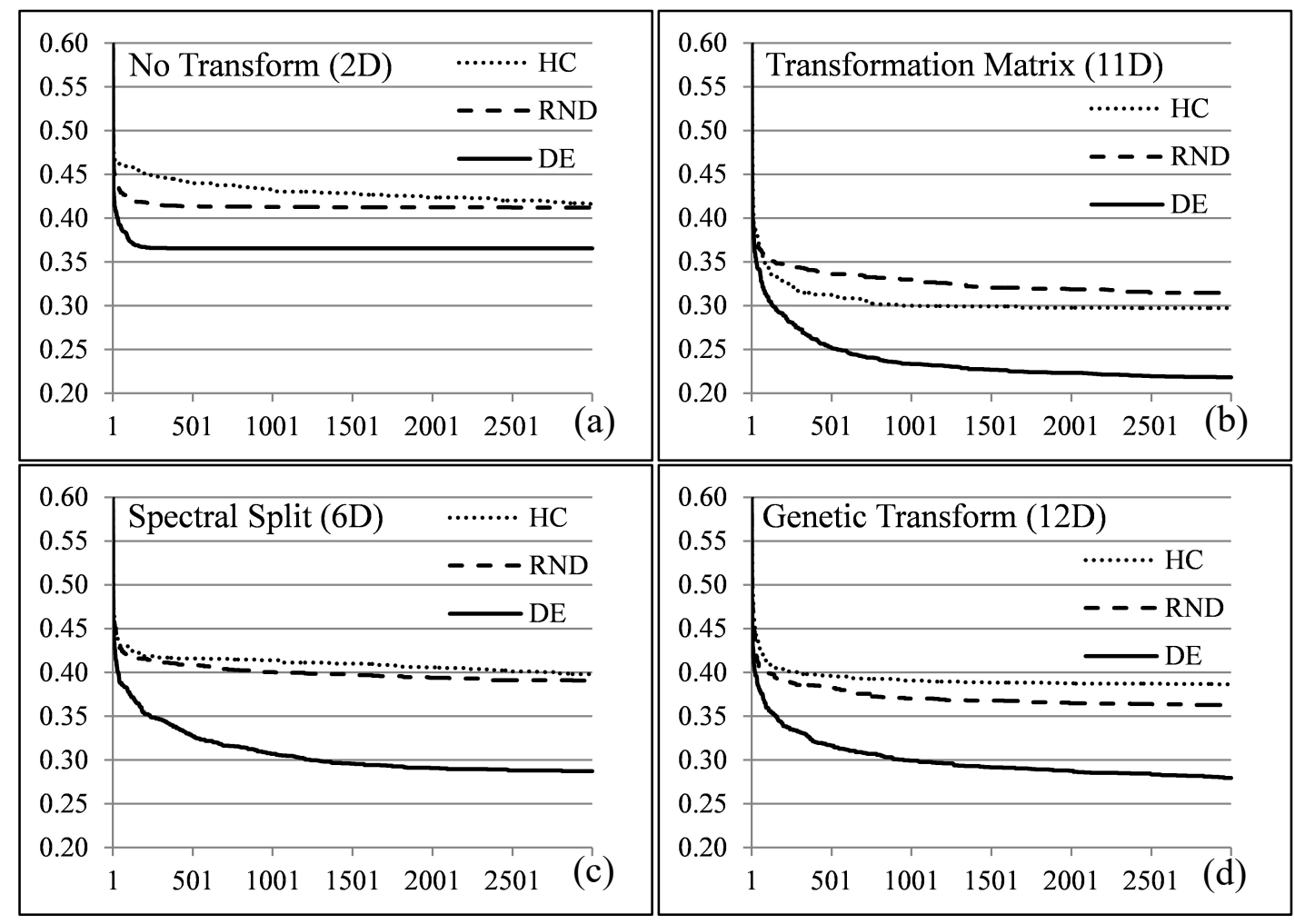

Figure 3.14. Fitness traces for three search methods on the Jowhaar site (SLIC segmentation) using the (a) No Transform, (b) Transformation Matrix, (c) Spectral Split and (d) Genetic Transform transformation strategies. The x-axes delineate search iterations. The y-axes delineate RWJ evaluation scores.

In all instances the differential evolution method gave the best final metric scores as well as maintaining the lowest values during the search process. This highlights some difficulty (discontinuities/ruggedness, deceptiveness, and multimodality) of the search landscapes (e.g., Figure 3.3), necessitating the use of more complex search strategies. Within a few generations, less than 250 search iterations, the DE search strategy starts to deliver meaningfully better results compared to the other two simple search strategies. As the dimensionality of the problem increases, in general, the magnitude of the differences in the results of the DE versus HC/RND increases. The advantages of using a more complex search strategy like DE is much more marginal when considering the variant of the method not employing any data transformation (Figures 3.13a and 3.14a).

Figure 3.15 shows fitness traces generated for the Jowhaar site using the SLIC and MS segmentation algorithms and four of the transformation function strategies. It is evident that simpler, or lower dimensional strategies (e.g., No Transform or Spectral Split) do not provide an initial performance increase over more complex, higher dimensional, variants (e.g., Transformation Matrix and Genetic Transform). This could be due to general usefulness of some transforms leading to better results by just randomly selecting points in the search space, with the magnitude in fitness differences increasing as the actual search progresses. Different fitness traces rarely cross, with the Transformation Matrix strategy, for both SLIC and MS, providing better final metric scores as well as maintaining the 
best results throughout the search process. Considering the MS segmentation algorithm, the No Transform, Spectral Split and Genetic Transform strategies have very similar fitness traces, but with no penalty for using the more complex variants.

Interestingly for this specific problem, the MS algorithm outperforms the SLIC algorithm under any transformation condition. Additionally, observing the fitness traces in this section, it could be argued that 1000 to 1500 search iterations are sufficient, keeping in mind the problems, metrics and transformation functions considered.

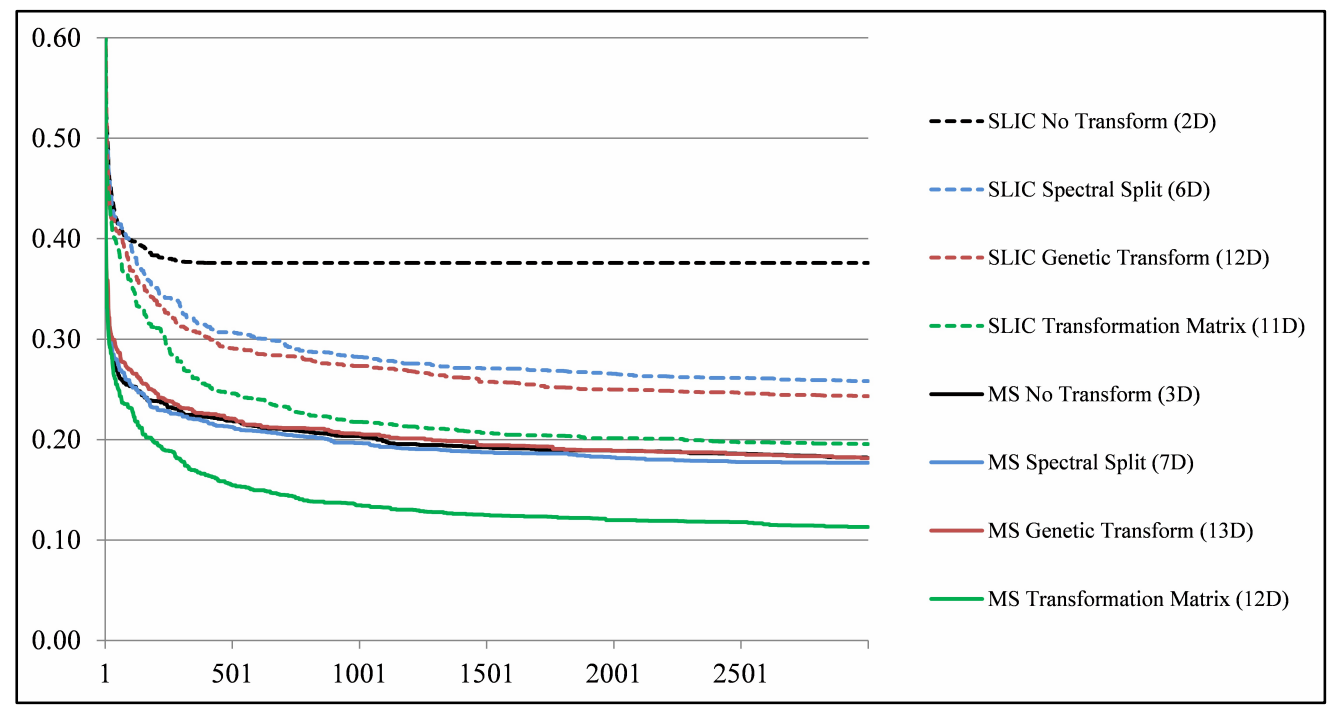

Figure 3.15. Fitness traces for the two tested segmentation algorithms (SLIC and MS) on the Jowhaar site using the different transformation function strategies. The $\mathrm{x}$-axes delineate search iterations. The y-axes delineate RWJ evaluation scores.

\subsubsection{Parameter Domain Interdependencies}

Tables 3.6 and 3.7 lists the parameters obtained for the MS and SLIC segmentation algorithms under different metric and data transformation conditions. The achieved fitness scores are also given. Examining Table 3.6, which denotes resultant parameters with the MS algorithm, very specific final fitness scores (low standard deviations) are generated by a wide range of different parameter sets under each data transformation condition. Thus multiple optima exist when considering the MS algorithm that approximates to the same (optimal) achievable segmentation quality, similar to the blue shaded cells illustrated for an arbitrary problem in Figure 3.3. None the less, various combinations of optimal scale parameter values achieved under different transformation conditions are statistically significantly different according to the student's t-test with a 95\% confidence interval (e.g., MS/PD_OCE No Transform and Spectral Split), suggesting different optimal values under different transformation conditions. 
Table 3.6. Multiresolution Segmentation (MS) segmentation algorithm parameters obtained over different metric and data transformation function conditions for the

Hagadera test site.

\begin{tabular}{ccccc}
\hline MS, PD_OCE & Fitness & Scale & Shape & Compactness \\
\hline No Transform & $0.78 \pm 0.02$ & $16.48 \pm 9.05$ & $0.12 \pm 0.11$ & $0.58 \pm 0.29$ \\
Spectral Split & $0.74 \pm 0.02$ & $12.06 \pm 6.37$ & $0.04 \pm 0.04$ & $0.54 \pm 0.38$ \\
Transformation Matrix & $0.62 \pm 0.02$ & $10.99 \pm 3.95$ & $0.09 \pm 0.07$ & $0.71 \pm 0.36$ \\
Genetic Contrast & $0.80 \pm 0.02$ & $15.98 \pm 8.56$ & $0.12 \pm 0.09$ & $0.65 \pm 0.40$ \\
Genetic Transform & $0.74 \pm 0.02$ & $11.61 \pm 4.29$ & $0.13 \pm 0.11$ & $0.67 \pm 0.39$ \\
MS, RWJ & & & & \\
No Transform & $0.49 \pm 0.02$ & $24.21 \pm 17.66$ & $0.13 \pm 0.22$ & $0.69 \pm 0.37$ \\
Spectral Split & $0.45 \pm 0.03$ & $15.30 \pm 5.57$ & $0.04 \pm 0.03$ & $0.66 \pm 0.33$ \\
Transformation Matrix & $0.39 \pm 0.03$ & $13.12 \pm 13.95$ & $0.12 \pm 0.26$ & $0.49 \pm 0.36$ \\
Genetic Contrast & $0.51 \pm 0.03$ & $18.16 \pm 10.60$ & $0.09 \pm 0.12$ & $0.71 \pm 0.30$ \\
Genetic Transform & $0.46 \pm 0.03$ & $18.22 \pm 16.08$ & $0.11 \pm 0.24$ & $0.54 \pm 0.38$ \\
\hline
\end{tabular}

Table 3.7. SLIC segmentation algorithm parameters obtained over different metric and data transformation function conditions for the Bokolmanyo test site.

\begin{tabular}{cccc}
\hline SLIC, PD_OCE & Fitness & Scale & Compactness \\
\hline No Transform & $0.78 \pm 0.00$ & $7.26 \pm 0.25$ & $27.40 \pm 0.00$ \\
Spectral Split & $0.64 \pm 0.04$ & $9.46 \pm 1.93$ & $31.17 \pm 9.76$ \\
Transformation Matrix & $0.50 \pm 0.03$ & $8.66 \pm 0.47$ & $36.02 \pm 6.39$ \\
Genetic Contrast & $0.75 \pm 0.03$ & $9.68 \pm 1.32$ & $27.69 \pm 8.23$ \\
Genetic Transform & $0.46 \pm 0.03$ & $7.68 \pm 0.54$ & $30.69 \pm 9.51$ \\
SLIC, RWJ & & & \\
No Transform & $0.33 \pm 0.01$ & $9.16 \pm 1.37$ & $38.03 \pm 0.77$ \\
Spectral Split & $0.32 \pm 0.04$ & $10.72 \pm 1.56$ & $38.56 \pm 1.96$ \\
Transformation Matrix & $0.23 \pm 0.02$ & $8.22 \pm 0.25$ & $38.22 \pm 2.83$ \\
Genetic Contrast & $0.42 \pm 0.02$ & $10.28 \pm 1.00$ & $37.22 \pm 6.02$ \\
Genetic Transform & $0.25 \pm 0.02$ & $9.42 \pm 1.02$ & $36.50 \pm 4.45$ \\
\hline
\end{tabular}

Table 3.8 lists the achieved scale parameter values over the allocated 25 runs using the RWJ metric and no data transformation as shown in Table 3.6. Note the two distinct value ranges, around 7-9 and again at 24 , resulting in the high standard deviation of 17.66 for the scale parameter, but still resulting in a fitness score with a standard deviation of less than 0.02 . Under all transformation conditions, low shape/color parameters are generated, with relatively low standard deviations. No significant conclusion can be made on the behavior of the shape/color and compactness/smoothness parameters.

Table 3.8. Scale parameter values obtained (sorted) from the results depicted in Table 3.6 with MS segmentation, no transformation and the Reference Weighted Jaccard (RWJ) metric.

\begin{tabular}{|c|c|c|c|c|c|c|c|c|c|c|c|c|}
\hline Scale & 7.8 & 7.8 & 7.8 & 7.8 & 9.2 & 9.2 & 9.2 & 10.6 & 21.8 & 21.8 & 24.6 & 24.6 \\
\hline 24.6 & 24.6 & 24.6 & 24.6 & 24.6 & 24.6 & 24.6 & 24.6 & 24.6 & 24.6 & 49.8 & 72.2 & 75 \\
\hline
\end{tabular}


Opposed to the results from the MS segmentation algorithm, the SLIC algorithm (Table 3.7) displays more specific scale and compactness parameter values as well as delivering very specific fitness scores. Again, various combinations of optimal scale parameter values achieved are statistically significantly different according to the student's t-test with a $95 \%$ confidence interval. This verifies, as with the MS algorithm, apparent parameter dependencies between the parameter domains of the segmentation algorithms and data transformation functions.

Finally Table 3.9 lists the results of the parameter interdependency test [50] performed on the Bokolmanyo site with the MS algorithm and Spectral Split combination under the RWJ metric condition. The parameters listed on the vertical axis are considered affected by the parameters listed horizontally if the result of an interdependency test is greater than zero. The table is divided into four quadrants, depicting the interactions of the two parameter domains. If both the top right and bottom left quadrants returned 0 for all cells, the two parameter domains can be considered as not interacting. In this simple example, all parameters are affected by all other parameters, to varying degrees. This suggests interdependencies. Interestingly, considering 100 evaluations for each cell, some interdependencies are frequent or strong (of no importance in determining interdependency). The scale parameter of the MS algorithm can be considered as its most important or sensitive parameter, reflected by the high degree by which its value is affected by all the other parameters. Note that these results reflect parameter interactions or the frequency of interactions, and not the contributions to segment quality of the interactions.

Table 3.9. Parameter domain interdependency test over 100 runs for the MS segmentation algorithm and Spectral Split transform on the Bokolmanyo site using the RWJ metric.

\begin{tabular}{ccccc|ccc}
\hline & B1 & B2 & B3 & Height & Scale & Shape & Compt \\
\hline B1 & & 22 & 27 & 26 & 4 & 9 & 39 \\
B2 & 24 & & 33 & 26 & 1 & 4 & 34 \\
B3 & 21 & 31 & & 30 & 3 & 4 & 31 \\
Height & 36 & 34 & 39 & & 3 & 7 & 34 \\
\hline Scale & 34 & 33 & 29 & 34 & & 40 & 39 \\
Shape & 24 & 29 & 35 & 36 & 28 & & 41 \\
Compt & 14 & 27 & 14 & 14 & 2 & 2 & \\
\hline
\end{tabular}

\subsection{Conclusions}

\subsubsection{Potential of Expanded Search Spaces in Sample Supervised Segment Generation}

In this work a sample supervised segment generation method was presented and profiled. Expanded search spaces were introduced in the optimization loop, here by adding low-level image processing functions. In the context of segmentation problems, such an expanded search landscape allows for creating closer correlations between thematic and spectral similarity, allowing the given segmentation algorithms to generate better quality segments. In addition, such an extension to an automatic segmentation algorithm parameter tuning system is simple to implement.

An interesting and sometimes advocated effect of such sample supervised optimization based approaches, is their potential to generate good or quality solutions in ways an expert user might never 
have foreseen. In our examples the results from Table 3.7 (also see Figure 3.12b) serve as a good example of such a scenario. Combining the MS segmentation algorithm with the Transformation Matrix, setting a low scale parameter (compared to the other variants) and creating washed-out monotone imagery works very well in numerous problem instances.

Four parameterized data transformation functions, having varied and sometimes complex internal characteristics have been tested with this method. Experimentation was conducted under various problem, metric, transformation function and segmentation algorithm conditions with generally favorable results advocating the utility of this method compared to simpler variants. Generalizability was also considered, with numerous reference segments tested and all results given as cross-validated. Care should be taken in selecting combinations of segmentation algorithms and data transformation functions, as a worsening of results are also possible under certain conditions. Such transformation functions or modifications of the data are exclusively used internally in the segmentation process and the resulting transformed data have no use for further analyses or visualization procedures.

The utility of using more complex search methods for traversing these enlarged search spaces was also investigated. It was shown that simpler search strategies, such as a single agent hill climber and random search, were inefficient in exploring the search landscape compared to a differential evolution search strategy. In addition, variants of the method having much more parameters and thus significantly higher dimensional search spaces do not have any initial disadvantage in the search process compared to simpler variants or the variant not performing any data transformation. This suggests, in this context, no search duration penalty in adding transformation functions. Finally, it is shown via optimal achieved segmentation parameters and a simple interdependency test that the low-level and mid-level processes investigated here are (highly) interdependent, necessitating simultaneous optimization of these two parameter domains.

The method described here can be used on its own if the considered problem is simple enough, keeping in mind the capabilities of the segmentation algorithm. This could be judged by the output metric scores. A very low score implies results are sufficiently good to use the segments as is. Otherwise, such an approach could form part of a more complete image analysis strategy, where the method is used to generate intermediary results, with other methods or processes performing more encompassing image analysis. For example in an expert systems approach (e.g., rule set development) segments can be generated as optimally as possible, quantitatively judged, for a certain land-cover type before more elaborate processes are used to split, merge and classify segments.

\subsubsection{Methodological Shortcomings and Open Questions for Future Research}

Although such a method shows promise, some shortcomings and open issues are highlighted here. Firstly, the number of training samples needed by such a method to generalize well is unknown. In this work reference segment sets contained between 28 and 40 references, thus with two-fold cross-validation between 14 and 20 references were used to train the model. In preliminary experimentation, reference sets containing less than 5-8 references displayed bad generalization performance. More complex and thus more flexible models (search landscapes) might fit closer to the training reference segments than simpler variants and have worse generalizability, unless generalizability tests such as cross-validation are explicitly implemented. 
Another open issue surrounding generalizability is how well different combinations of segmentation algorithms and low-level image processing generalizes over a range of problems, something not explicitly tested here. It could be stated that, considering the conditions in this study, the MS segmentation algorithm with the Genetic Transform and Transformation Matrix functions do perform well over a range of problems. Different low-level image processing functions might be suited to different problem types or categories. Future work could investigate additional promising functions used in combination with commonly used segmentation algorithms. Neighborhood based transforms might be worth investigating. Search landscape dimensionality should be kept in mind, as enlarged search spaces are more difficult to traverse.

The computationally expensive nature of image segmentation necessitates the efficient traversal of the search landscape. Such a sample supervised strategy advocates user interaction, so the method should ideally complete in a time frame acceptable to a user engaged with the image analysis. The computing time for a fitness evaluation iteration in this work (excluding Genetic Contrast) ranges from $0.10 \pm 0.01$ seconds (SLIC segmentation, 28 subsets from the Bokolmanyo site, No Transform) to $3.36 \pm 0.28$ seconds (MS segmentation, 40 subsets from the Akonolinga site, Genetic Transform) on an Intel Xeon E5-2643 3.5 GHz processor using single threaded processing. Running fitness evaluations (subset segmentation, transforms, fitness calculation) on a parallel computing architecture could be considered. In addition, motivated by the computationally expensive nature of fitness evaluation, more careful consideration could be given to the choice of the optimizer. Performing rigid meta-optimization (not conducted in this work), using metaheuristics with self-adapting metaparameters or hyperheuristics could be considered for further investigation.

The choice of an optimal metric is, in our opinion, an open issue. In this work four different metrics, which are able to measure over- and under-segmentation were used. In addition to the general "area-overlap" metrics tested here, metrics observing spectral characteristics and boundary offsets could be considered. Metrics sensitive to user inaccuracies in delineating reference segments could also be considered. It is still unclear how different metrics relate or correlate to final classification accuracies. Multi-objective optimization may also be considered.

Finally, the optimization concept of "epistatic links" (parameter interdependencies) [51] could, in some sense, be applied to some interactions in GEOBIA processes [8,9]. In this work an interaction between two domains was investigated and profiled. Investigating the potential of modelling classical GEOBIA processes as complex optimization problems could be profitable. Quality can be measured via empirical discrepancy methods, empirical goodness methods and even traditional notions of classifier accuracies.

\section{Acknowledgments}

This work has been conducted under the GIONET project funded by the European Commission, Marie Curie Programme, Initial Training Networks, Grant Agreement number PIT-GA-2010-264509. The programming libraries OpenCV, TerraLib and SwarmOps were used in this work.

\section{Author Contributions}

Christoff Fourie conceived the concept presented, implemented the methodologies, conducted the experimentation and wrote the manuscript. Elisabeth Schoepfer contributed to revision and editing. 


\section{Conflicts of Interest}

The authors declare no conflict of interest.

\section{References and Notes}

1. Addink, E.A.; van Coillie, F.M.B.; de Jong, S.M. Introduction to the GEOBIA 2010 special issue: From pixels to geographic objects in remote sensing image analysis. Int. J. Appl. Earth Observ. Geoinf. 2012, 15, 1-6.

2. Blaschke, T. Object based image analysis for remote sensing. ISPRS J. Photogramm. Remote Sens. 2010, 65, 2-16.

3. Hay, G.J.; Castilla, G. Geographic Object-Based Image Analysis (GEOBIA): A New Name for A New Discipline. In Object-Based Image Analysis: Spatial Concepts for Knowledge-Driven Remote Sensing Applications; Blaschke, T., Lang, S., Hay, G.J., Eds.; Springer: Berlin, Germany, 2008; pp. 75-90.

4. Blaschke, T.; Hay, G.J.; Kelly, M.; Lang, S.; Hofmann, P.; Addink, E.; Feitosa, R.Q.; van der Meer, F.; van der Werff, H.; van Coillie, F.; et al. Geographic object-based image-Towards a new paradigm. ISPRS J. Photogramm. Remote Sens. 2014, 87, 180-191.

5. Atkinson, P.M.; Aplin, P. Spatial variation in land cover and choice of spatial resolution for remote sensing. Int. J. Remote Sens. 2004, 25, 3687-3702.

6. Hay, G.J.; Castilla, G.; Wulder, M.A.; Ruiz, J.R. An automated object-based approach for the multiscale image segmentation of forest scenes. Int. J. Appl. Earth Observ. Geoinfor. 2005, 7, 339-359.

7. Drăguţ, L.; Tiede, D.; Levick, S.R. ESP: A tool to estimate scale parameter for multiresolution image segmentation of remotely sensed data. Int. J. Geogr. Inf. Sci. 2010, 24, 859-871.

8. Baatz, M.; Hoffman, C.; Willhauck, G. Progressing from Object-Based to Object-Oriented Image Analysis. In Object-Based Image Analysis: Spatial Concepts for Knowledge-Driven Remote Sensing Applications; Blaschke, T., Lang, S., Hay, G.J., Eds.; Springer: Berlin, Germany, 2008; pp. 29-42.

9. Lübker, T.; Schaab, G. A work-flow design for large-area multilevel GEOBIA: Integrating statistical measures and expert knowledge. Int. Arch. Photogramm. Remote Sens. Spat. Inf. Sci. 2010. Available online: http://www.isprs.org/proceedings/XXXVIII/4-C7/pdf/luebkerT.pdf (accessed on 23 April 2014).

10. Baatz, M.; Schäpe, A. Multiresolution Segmentation: An Optimization Approach for High Quality Multi-Scale Image Segmentation. In Angewandte Geographische Informationsverarbeitung; Strobl, J., Blaschke, T., Griesebner, G., Eds.; Wichmann Verlag: Karlsruhe, Germany, 2000; Volume 12, pp. 12-23.

11. Castilla, G.; Hay, G.J.; Ruiz-Gallardo, J.R. Size-constrained region merging (scrm): An automated delineation tool for assisted photointerpretation. Photogramm. Eng. Remote Sens. 2008, 74, 409-419.

12. Lang, S.; Langanke, T. Object-based mapping and object-relationship modeling for land use classes and habitats. Photogramm. Fernerkund. Geoinf. 2006, 10, 5-18. 
13. Zhang, Y.J. A survey on evaluation methods for image segmentation. Pattern Recognit. 1996, 29, $1335-1346$.

14. Bhanu, B.; Lee, S.; Ming, J. Adaptive image segmentation using a genetic algorithm. IEEE Trans. Syst. Man Cybern. 1995, 25, 1543-1567.

15. Feitosa, R.Q.; Ferreira, R.S.; Almeida, C.M.; Camargo, F.F.; Costa, G.A.O.P. Similarity metrics for genetic adaptation of segmentation parameters. Int. Arch. Photogramm. Remote Sens. Spat. Inf. Sci. 2010. Available online: http://www.isprs.org/proceedings/XXXVIII/4-C7/pdf/Feitosa_150.pdf (accessed on 23 April 2014).

16. Persello, C.; Bruzzone, L. A novel protocol for accuracy assessment in classification of very high resolution images. IEEE Trans. Geosci. Remote Sens. 2010, 48, 1232-1244.

17. Polak, M.; Zhang, H.; Pi, M. An evaluation metric for image segmentation of multiple objects. Image Vis. Comput. 2009, 27, 1223-1227.

18. Bartz-Beielstein, T. Experimental Research in Evolutionary Computation; Springer: Berlin, Germany, 2006.

19. Cagnoni, S. Evolutionary Computer Vision: A Taxonomic Tutorial. In Proceedings of Eighth International Conference on Hybrid Intelligent Systems, Barcelona, Spain, 10-12 September 2008.

20. Harding, S.; Leitner, J.; Schmidhuber, J. Cartesian Genetic Programming for Image Processing. In Genetic Programming Theory and Practice X; Riolo, R., Vladislavleva, E., Ritchie, M.D., Moore, J.H., Eds.; Springer: New York, NY, USA, 2013; pp. 31-44.

21. Yoda, I.; Yamamoto, K.; Yamada, H. Automatic acquisition of hierarchical mathematical morphology procedures by genetic algorithms. Image Vis. Comput. 1999, 17, 749-760.

22. Ebner, M. A Real-Time Evolutionary Object Recognition System. In Proceedings of 12th European Conference, EuroGP 2009 Tübingen, Tübingen, Germany, 15-17 April 2009.

23. Rosin, P.; Hervás, J. Image Thresholding for Landslide Detection by Genetic Programming. In Proceedings of First International Workshop on Multitemp, Trento, Italy, 13-14 September 2001.

24. Wang, J.; Tan, Y. A Novel Genetic Programming Algorithm for Designing Morphological Image Analysis Method. In Proceedings of International Conference on Swarm Intelligence, Chongqing, China, 12-15 June 2011.

25. Feitosa, R.Q.; Costa, G.A.O.P.; Cazes, T.B.; Feijo, B. A genetic approach for the automatic adaptation of segmentation parameters. Int. Arch. Photogramm. Remote Sens. Spat. Inf. Sci. 2006. Available online: http://www.isprs.org/proceedings/xxxvi/4-c42/Papers/ 11_Adaption\%20and\%20further\%20development\%20III/OBIA2006_Feitosa_et_al.pdf (accessed on 23 April 2014).

26. Derivaux, S.; Forestier, G.; Wemmert, C.; Lefèvre, S. Supervised image segmentation using watershed transform, fuzzy classification and evolutionary computation. Pattern Recognit. Lett. 2010, 31, 2364-2374.

27. Pignalberi, G.; Cucchiara, R.; Cinque, L.; Levialdi, S. Tuning range image segmentation by genetic algorithm. EURASIP J. Appli. Sig. Process. 2003, doi:10.1155/S1110865703303087.

28. Martin, V.; Thonnat, M. A cognitive vision approach to image segmentation. Tool. Artif. Intell. 2008, 1, 265-294. 
29. Ferreira, R.S.; Feitosa, R.Q.; Costa, G.A.O.P. A Multiscalar, Multicriteria Approach for Image Segmentation. In Proceedings of Geographic Object-Based Image Analysis (GEOBIA) 2012, Rio de Janeiro, Brazil, 7-9 May 2012.

30. Happ, P.; Feitosa, R.Q.; Street, A. Assessment of Optimization Methods for Automatic Tuning of Segmentation Parameters. In Proceedings of Geographic object-based image analysis (GEOBIA) 2012, Rio de Janeiro, Brazil, 7-9 May 2012.

31. Freddrich, C.M.B.; Feitosa, R.Q. Automatic adaptation of segmentation parameters applied to non-homogeneous object detection. Int. Arch. Photogramm. Remote Sens. Spat. Inf. Sci. 2008. Available online: http://www.isprs.org/proceedings/XXXVIII/4-C1/Sessions/Session6/ 6705_Feitosa_Proc_pap.pdf (accessed on 23 April 2014).

32. Michel, J.; Grizonnet, M.; Canevet, O. Supervised Re-Segmentation for Very High-Resolution Satellite Images. In Proceedings of 2012 IEEE International Geoscience and Remote Sensing Symposium (IGARSS 2012), Munich, Germany, 22-27 July 2012.

33. Levin, A.; Weiss, Y. Learning to combine bottom-up and top-down segmentation. Int. J. Comput. Vis. 2009, 81, 105-118.

34. Li, C.; Kowdle, A.; Saxena, A.; Chen, T. Toward holistic scene understanding: Feedback enabled cascaded classification models. IEEE Trans. Pattern Anal. Mach. Intell. 2012, 34, 1394-1408.

35. Borenstein, E.; Ullman, S. Class-Specific, Top-Down Segmentation. In Computer Vision-ECCV 2002; Heyden, A., Sparr, G., Nielsen, M., Johansen, P., Eds.; Springer: Berlin, Germany, 2002; pp. 109-122.

36. Hoiem, D.; Efros, A.A.; Hebert, M. Closing the Loop in Scene Interpretation. In Proceedings of IEEE Conference on Computer Vision and Pattern Recognition (CVPR 2008), Anchorage, AK, USA, 24-26 June 2008.

37. Zheng, S.; Yuille, A.; Tu, Z. Detecting object boundaries using low-, mid-, and high-level information. Comput. Vis. Image Underst. 2010, 114, 1055-1067.

38. Leibe, B.; Schiele, B. Interleaving Object Categorization and Segmentation. In Cognitive Vision Systems: Sampling the Spectrum of Approaches; Christensen, H.I., Nagel, H., Eds.; Springer: Berlin, Germany, 2006; pp. 145-161.

39. He, X.; Zemel, R.S.; Ray, D. Learning and Incorporating Top-Down Cues in Image Segmentation. In Computer Vision-ECCV 2006; Leonardis, A., Bischof, H., Pinz, A., Eds.; Springer: Berlin, Germany, 2006; pp. 338-351.

40. Schnitman, Y.; Caspi, Y.; Cohen-Or, D.; Lischinski, D. Inducing Semantic Segmentation from An Example. In Computer Vision-ACCV 2006; Narayanan, P.J., Nayar, S.K., Shum, H., Eds.; Springer: Berlin, Germany, 2006; pp. 373-384.

41. Daelemans, W.; Hoste, V.; de Meulder, F.; Naudts, B. Combined Optimization of Feature Selection and Algorithm Parameters in Machine Learning of Language. In Machine Learning: Ecml 2003; Springer: Berlin, Germany, 2003; pp. 84-95.

42. Lin, S.-W.; Ying, K.-C.; Chen, S.-C.; Lee, Z.-J. Particle swarm optimization for parameter determination and feature selection of support vector machines. Expert Syst. Appl. 2008, 35, $1817-1824$. 
43. Fourie, C.; Van Niekerk, A.; Mucina, L. Optimising a One-Class SVM for Geographic Object-Based Novelty Detection. In Proceedings of AfricaGeo, Cape Town, South Africa, 31 May-2 June 2011.

44. Chong, H.Y.; Gortler, S.J.; Zickler, T. A perception-based color space for illumination-invariant image processing. ACM Trans. Gr. 2008. Available online: http://gvi.seas.harvard.edu/sites/ all/files/Color_SIGGRAPH2008.pdf (accessed on 23 April 2014).

45. Shan, Y.; Yang, F.; Wang, R. Color Space Selection for Moving Shadow Elimination. In Proceedings of Fourth International Conference on Image and Graphics (ICIG 2007), Chengdu, China, 22-24 August 2007.

46. Trias-Sanz, R.; Stamon, G.; Louchet, J. Using colour, texture, and hierarchial segmentation for high-resolution remote sensing. ISPRS J. Photogramm. Remote Sens.2008, 63, 156-168.

47. Kwok, N.; Ha, Q.; Fang, G. Effect of Color Space on Color Image Segmentation. In Proceedings of Image and Signal Processing (CISP 2009), Tianjin, China, 17-19 October 2009.

48. Munteanu, C.; Rosa, A. Towards Automatic Image Enhancement Using Genetic Algorithms. In Proceedings of Evolutionary Computation (CEC 2000), La Jolla, CA, USA, 16-19 July 2000.

49. Shyu, M.; Leou, J. A genetic algorithm approach to color image enhancement. Pattern Recognit. 1998, 31, 871-880.

50. Sun, L.; Yoshida, S.; Cheng, X.; Liang, Y. A cooperative particle swarm optimizer with statistical variable interdependence learning. Inf. Sci. 2012, 186, 20-39.

51. Weicker, K.; Weicker, N. On the Improvement of Coevolutionary Optimizers by Learning Variable Interdependencies. In Proceedings of Evolutionary Computation (CEC 1999), Washington, DC, USA, 6-9 July 1999.

52. Fourie, C.; Schoepfer, E. Combining the Heuristic and Spectral Domains in Semi-Automated Segment Generation. In Proceedings of Geographic Object-based Image Analysis (GEOBIA 2012), Rio De Janeiro, Brazil, 7-9 May 2012.

53. Storn, R.; Price, K. Differential evolution-A simple and efficient heuristic for global optimization over continuous spaces. J. Glob. Optim. 1997, 11, 341-359.

54. Melo, L.M.; Costa, G.A.O.P.; Feitosa, R.Q.; da Cruz, A.V. Quantum-inspired evolutionary algorithm and differential evolution used in the adaptation of segmentation parameters. Int. Arch. Photogramm. Remote Sens. Spat. Inf. Sci. 2008. Available online: http://www.isprs.org/ proceedings/xxxviii/4-c1/sessions/Session11/6667_Melo_Proc_pap.pdf (accessed on 23 April 2014).

55. Gorai, A.; Ghosh, A. Gray-Level Image Enhancement by Particle Swarm Optimization. In Proceedings of Nature \& Biologically Inspired Computing (NaBIC 2009), Coimbatore, India, 9-11 December 2009.

56. Pal, S.K.; Bhandari, D.; Kundu, M.K. Genetic algorithms for optimal image enhancement. Pattern Recognit. Lett. 1994, 15, 261-271.

57. Achanta, R.; Shaji, A.; Smith, K.; Lucchi, A.; Fua, P.; Susstrunk, S. Slic superpixels compared to state-of-the-art superpixel methods. IEEE Trans. Pattern Anal. Mach. Intell. 2012, 34, 2274-2282.

58. Jaccard, P. Distribution de la flore alpine: Dans le bassin des dranses et dans quelques régions voisines. Bulletin de la Société Vaudoise des Sciences Naturelles 1901, 37, 241-272. 
(C) 2014 by the authors; licensee MDPI, Basel, Switzerland. This article is an open access article distributed under the terms and conditions of the Creative Commons Attribution license (http://creativecommons.org/licenses/by/3.0/). 


\title{
CHAPTER 4: ON ATTRIBUTE THRESHOLDING AND DATA MAPPING FUNCTIONS IN A SUPERVISED CONNECTED COMPONENT SEGMENTATION FRAMEWORK
}

\author{
Fourie, C \\ German Remote Sensing Data Center (DFD), German Aerospace Center (DLR), \\ 82234 Oberpfaffenhofen.
}

Published in:

Remote Sensing (2015), 7, 7350-7377

Received: 17 March 2015 / Accepted: 27 May 2015 / Published: 3 June 2015

doi: $10.3390 /$ rs 70607350 
Remote Sens. 2015, 7, 1-x manuscripts; doi:10.3390/rs70x000x

Article

\section{On Attribute Thresholding and Data Mapping Functions in a Supervised Connected Component Segmentation Framework}

\section{Christoff Fourie}

German Aerospace Center (DLR), German Remote Sensing Data Center (DFD), 82234 Oberpfaffenhofen, Germany; E-Mail: christoffel.fourie@dlr.de, fourie.christoff@gmail.com; Tel.: +27-72-082-4528; Fax: +49-8153-28-1445.

Academic Editors: Arko Lucieer and Prasad S. Thenkabail

Received: 17 March 2015 / Accepted: 27 May 2015 / Published: 3 June 2015

\section{Abstract}

Search-centric, sample supervised image segmentation has been demonstrated as a viable general approach applicable within the context of remote sensing image analysis. Such an approach casts the controlling parameters of image processing — generating segments —as a multidimensional search problem resolvable via efficient search methods. In this work, this general approach is analyzed in the context of connected component segmentation. A specific formulation of connected component labeling, based on quasi-flat zones, allows for the addition of arbitrary segment attributes to contribute to the nature of the output. This is in addition to core tunable parameters controlling the basic nature of connected components. Additional tunable constituents may also be introduced into such a framework, allowing flexibility in the definition of connected component connectivity, either directly via defining connectivity differently or via additional processes such as data mapping functions. The relative merits of these two additional constituents, namely the addition of tunable attributes and data mapping functions, are contrasted in a general remote sensing image analysis setting. Interestingly, tunable attributes in such a context, conjectured to be safely useful in general settings, were found detrimental under crossvalidated conditions. This is in addition to this constituent's requiring substantially greater computing time. Casting connectivity definitions as a searchable component, here via the utilization of data mapping functions, proved more beneficial and robust in this context. The results suggest that further investigations into such a general framework could benefit more from focusing on the aspects of data mapping and modifiable connectivity as opposed to the utility of thresholding various geometric and spectral attributes. 
Keywords: geographic object-based image analysis; segmentation; mathematical morphology; sample supervised; spatial metrics; metaheuristics; connected component

\subsection{Introduction}

Research into operational remote sensing image analysis methodologies continues to enjoy attention in response to real-world requirements within the private and public sectors. This is evident in the variety of journals, conferences, and workshops within the context of remote sensing methodology, with a concomitant increase in niche interdisciplinary sub-disciplines. Two examples, dealing mainly with optical data, are Geographic Object-Based Image Analysis (GEOBIA) [1] and mathematical imaging within remote sensing [2]. This contribution falls within the context of these two subdisciplines.

A new variant of sample supervised segment generation is analyzed, recently presented as a conference contribution [3]. Sample supervised segment generation denotes a general search-centric methodological framework for generating quality image segments based on the provision of a selection of exemplar segments [4-7]. Segments generated via such an approach may be used in further processes to progress to a final information product. A connected component (quasi-flat zone) segmentation algorithm, specifically an attribute-enhanced variant of Constrained Connectivity (CC) [8-11], is embedded into such a sample supervised segment generation framework. The modular and extendable nature of the segmentation algorithm allows for the definition of arbitrary attribute criteria to assist in shaping the nature of the generated segments. Such tunable attribute criteria are cast as an additional parameter constituent within the sample supervised segment generation framework. Additionally, data transformation or mapping functions [6] may be added as a constituent in such a framework. Utilizing mapping functions falls within the context of defining connected components more elaborately, also considered for connected component segmentation algorithms via additional processing (pre-filtering/post-filtering) [12,13], via analyzing scene-wide statistics [14,15], and via the notion of hyperconnections [16-18]. Adding mapping functions into such a framework results in three distinct, interdependent parameter constituents that need consideration.

The contribution of this work is two-fold. Firstly, the feasibility of the proposed framework is analyzed to demonstrate that it constitutes a valid optimization problem, having interacting constituents and being searchable via metaheuristics. The presented method may be cast as four separate variants, using various constituent combinations. Secondly and primarily, the relative merits of the two added constituents are contrasted, namely that of additional mapping functions and that of tunable attribute criteria. It is demonstrated in a selection of remote sensing image analysis problems, under various metric conditions and also under cross-validated conditions, that utilizing geometric and attribute criteria to shape the nature of the generated segments may be detrimental. This suggests more careful consideration in the utilization of attributes in the context of such a framework.

This paper is structured as follows. Section 4.2 presents an overview and review of background principles related to the segmentation approach used, as well as some aspects of a sample supervised segment generation framework. In Section 4.3, the investigated method and its variants are detailed. The data used are described in Section 4.4. In Section 4.5, the method is experimentally evaluated and 
its variants are contrasted. Concluding comments and prospects for future work are given in Section 4.6 .

\subsection{Background and Related Work}

\subsubsection{Graph-Based Connected Component Segmentation}

Graph-based connected component segmentation defines a family of image-processing methods stemming from work within the broader context of mathematical morphology. See [19] for a general overview of mathematical morphology and [20] for an overview of segmentation concepts and approaches. Developments and applications within remote sensing mirror the advancements of basic research within mathematical morphology. Classic mathematical morphology tools (erosion, dilation, and reconstruction filtering) [19] found successful applications within remote sensing, e.g., [21,22].

A major development in the proliferation of applications of mathematical morphology tools in remote sensing is the notion of a flat zone. A flat zone defines a specific level in a hierarchical image partitioning based on grey-level intensities [20,23,24]. Various successful methods and applications have been presented based on this notion, e.g., [2,25-28]. This development was elaborated upon with the notion of a quasi-flat zone, which relaxes the restrictive definition of a flat zone by introducing a certain level of dissimilarity tolerance between pixels (parameter controlled) [8,9,20,29]. Quasi-flat zones form the basis of flexible segmentation algorithms, suited to remote sensing image analysis problems. Resultant segmentation algorithms have attractive properties, including ease of extensibility and inherent modularity [16,29], computational and memory efficiency [9,30,31] (especially in hierarchical formulations), uniqueness (same segmentation on repeated runs, some formulations [8]), and mathematically rigorous formulations. Efficient data structures and computational efficiencies are major considerations in such approaches. Comparative and practical analyses with other commonly used segmentation algorithms within remote sensing are needed, e.g., [32].

\subsubsection{Constrained Connectivity}

Constrained Connectivity (CC) is an image partitioning or simplification (segmentation) method based on the identification of quasi-flat zone connected components [8,10,11,13,29]. Spectral difference or grey-level difference is defined as a connectivity relation, denoted by $\alpha$ (alpha) and called the local range (parameter). Other constraints may be introduced. This notion was originally developed to address the chaining effect of the single linkage clustering method [8]. Quasi-flat zone approaches may be considered an alternative or extension of the mathematical morphology approaches applied in remote sensing that considers image local extrema in processing, e.g., [23,33,34]. The algorithm is hierarchical, with a fully calculated hierarchy known as an alpha tree. Within an alpha tree, all constraints (parameter combinations) are calculated and stored in a tree data structure [9,31], with connected components efficiently computed via Tarjan's union-find algorithm [9,31] (or others).

CC may be defined as the partitioning of an image into $\alpha$-connected (alpha) components. Two pixels are considered connected ( $\alpha$-connected) if there exists a path between them such that the greylevel difference of successive pixels in this path does not exceed a given value $(\alpha)$. The connected component (segment) of a given pixel $p$ and other pixels $q$ for a given $\alpha$ may be denoted as follows: 


$$
\begin{aligned}
\alpha-C C(p)= & \{p\} \cup\left\{q \mid \text { a path } S=\left(p=p_{1}, \ldots, p_{n}=q\right), n>1, \text { such that } D\left(p_{i}, p_{i+1}\right)\right. \\
& \leq \alpha \text { for all } 1 \leq i<n\}
\end{aligned}
$$

$\mathrm{D}$ denotes a range function that calculates the difference between the spectral values of two given connected pixels. Note that successive segmentations of $\alpha-\mathrm{CC}$ are hierarchical (increasing $\alpha$ values) and that a unique partition (identical over various runs) is generated. Various approaches [8,31] may be followed for computing an $\alpha$-CC segmentation efficiently, with a priority queue approach followed here [8].

A useful additional parameter, namely the global range criterion (w), may also be introduced. $w$ constrains the creation of connected components by limiting the maximum spectral difference between any two pixels in a connected component. Additional constraining attributes (increasing/nonincreasing) ("Attr") may also be further introduced via predicates evaluating the potential connected components. The connected component of a pixel may then be described as:

$$
\begin{gathered}
(\alpha, w)-C C(p)=\{p\} \cup\left\{q \mid \text { a path } S=\left(p=p_{1}, \ldots, p_{n}=q\right), n>\right. \\
\left.1, \text { such that } D\left(p_{i}, p_{i+1}\right) \leq \alpha \text { for all } 1 \leq i<n \text { and } \operatorname{Max}(S)-\operatorname{Min}(S) \leq w\right\}
\end{gathered}
$$

with a further predicate $(\mathrm{P})$ able to restrict the growth defined for a given connected component $(\mathrm{X})$ as:

$$
P((\alpha, w)-C C(p))=\text { true if } \operatorname{Attr}(X) \leq t, \text { false otherwise }
$$

where $t$ denotes a threshold value for a given attribute (e.g., segment area) and Attr a function calculating the value of the attribute. For example, during the computation of connected components, if a given potential connected component (e.g., X) satisfies the local and global range criteria ( $\alpha, w)$, but the calculated area attribute of the potential connected component exceeds a given threshold value (t), it is not substantiated. If the calculated area attribute is below the given threshold value, the connected component is substantiated (a visual example of constraining attributes is given in Section 4.3).

$\operatorname{Max}(\mathrm{S})$ and $\operatorname{Min}(\mathrm{S})$ return the maximum and minimum spectral values within path $\mathrm{S}$, respectively. Various approaches exist to handle multichannel imagery $[8,35,36]$. Here a constraint is simply triggered for the entire given image (all bands) if any of the composite bands trigger a constraint.

Figure 4.1 illustrates an abstract image, with pixel values labeled with their intensity values, as well as shaded for easier visualization (lower value $=$ brighter pixel). Bold lines delineate connected component borders. Figure 4.1 a shows an image segmented $(\alpha w-\mathrm{CC})$ with $(0,0)-\mathrm{CC},(1,1)$-CC (Figure 4.1b), (1,2)-CC (Figure 4.1c), and (2,3)-CC (Figure 4.1d). Dotted lines denote an absence of a path between two pixels for the given constraints.

Figure 4.2 shows segmentation results using $\alpha \mathrm{w}-\mathrm{CC}$ on a subset of a real image (arbitrary) to highlight general characteristics. Transition regions [10] are characterized by multiple single pixel connected components in areas where the transition between homogeneous areas spans several pixels. Some approaches may be applied to diminish this effect [10,12] (Figure 4.2c-Iterative area filtering), not considered here owing to experimental anomalies observed with such additional processing. Post-processing may simply be applied to remove single pixels (among others). A structure is delineated with a red polyline (example element of interest) (a), with segmentation results shown using $(25,75)-\mathrm{CC}$ (b), a pre-processed [10] image segmented with $(25,75)$-CC (c) to minimize the effect of transition regions and $(50,200)-\mathrm{CC}(\mathrm{d})$. 


\begin{tabular}{|l|l|l|ll|l|}
\hline 5 & 4 & 1 & 4 & 4 & 1 \\
\cline { 5 - 6 } 2 & 1 & 1 & 2 & 2 & 2 \\
\cline { 1 - 3 } 2 & 3 & 1 & 2 & 2 & 3 \\
\hline 3 & 5 & 5 & 4 & 4 & 2 \\
\cline { 1 - 1 } 2 & 5 & 5 & 4 & 4 & 2 \\
\cline { 1 - 1 } 1 & 5 & 4 & 2 & 2 & 2 \\
\hline
\end{tabular}

(a)

\begin{tabular}{|ll|l|ll|l|}
\hline 5 & 4 & 1 & 4 & 4 & 1 \\
\cline { 1 - 2 } 2 & 1 & 1 & 2 & 2 & 2 \\
2 & 3 & 1 & 2 & 2 & 3 \\
\cline { 3 - 5 } 3 & 5 & 5 & 4 & 4 & 2 \\
2 & 5 & 5 & 4 & 4 & 2 \\
1 & 5 & 4 & 2 & 2 & 2 \\
\hline
\end{tabular}

(c)

\begin{tabular}{|l|l|l|ll|l|}
\hline 5 & 4 & 1 & 4 & 4 & 1 \\
\cline { 1 - 3 } 2 & 1 & 1 & 2 & 2 & 2 \\
\cline { 1 - 1 } 2 & 3 & 1 & 2 & 2 & 3 \\
\hline 3 & 5 & 5 & 4 & 4 & 2 \\
\cline { 1 - 1 } 2 & 5 & 5 & 4 & 4 & 2 \\
\hline 1 & 5 & 4 & 2 & 2 & 2 \\
\hline
\end{tabular}

(b)

\begin{tabular}{|lllllll|}
\hline 5 & 4 & 1 & 4 & 4 & 1 \\
\hline 2 & 1 & 1 & 2 & 2 & 2 \\
2 & 3 & 1 & 2 & 2 & 3 \\
3 & 5 & 5 & 4 & 4 & 2 \\
2 & 5 & 5 & 4 & 4 & 2 \\
1 & 5 & 4 & 2 & 2 & 2 \\
\hline
\end{tabular}

(d)

Figure 4.1. An abstract image, segmented with the $\alpha w-C C$ method illustrating its general characteristics, with (0,0)-CC shown in (a), (1,1)-CC in (b), (1,2)-CC in (c), and (2,3)-CC in $(d)$.

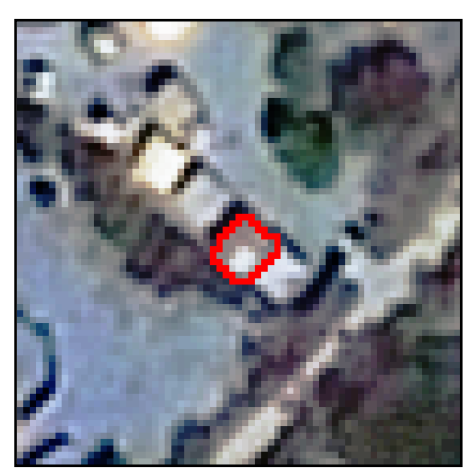

(a)

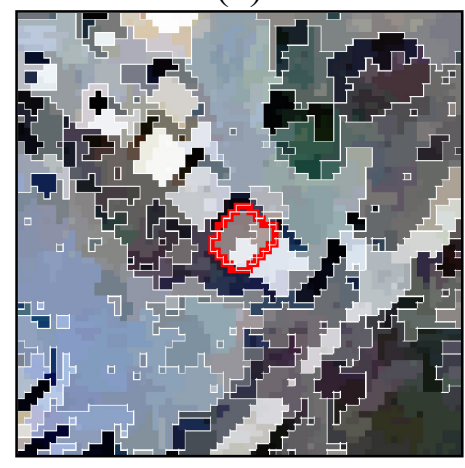

(c)

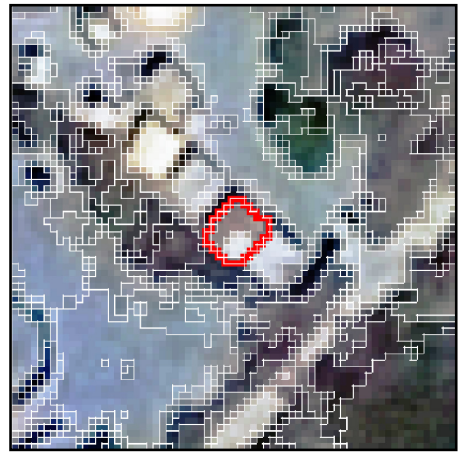

(b)

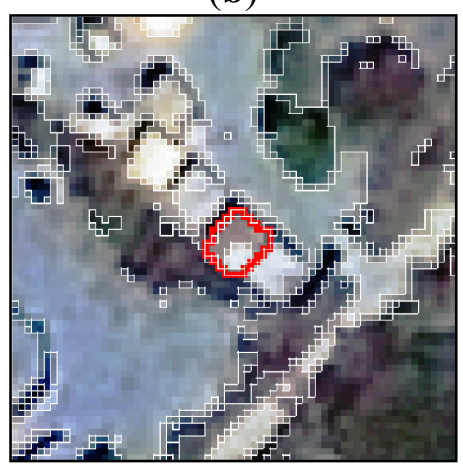

(d)

Figure 4.2. An image subset (a) segmented with ( $\alpha w-C C)$ to show its common characteristics on real imagery, with the local and global range parameters set to 25 and 75 (b), 25 and 75 with a region growing filter (c), and to 50 and 200 (d), respectively. The red polyline indicates an example element a user might be interested in. 


\subsubsection{Metaheuristics}

Metaheuristics constitutes a class of optimization algorithms (minimization or maximization) which are commonly multi-agent and stochastic in nature, and contain elements of search intensification and search diversification (see e.g., [37] for an overview). Many metaheuristic algorithm designs are inspired by naturally occurring search processes. Their main practical utility is in reducing computing costs to obtain optimal, or, more typically, near optimal results. Their application needs careful consideration in the choice of metaheuristic to be used (applicability, no free lunch theorem, search landscape characteristics), the handling of meta-parameters (meta-optimization, self-adaptation, empirical tuning, hyper-heuristics) and the nature of statistical evaluations and reporting being some examples [38-42]. Their utility in a certain context may be evaluated via experimentation [39], with robustness, absolute and relative results, standard deviation, required computing times, and search process characterization some of the measurable aspects. Their application in remote sensing image analysis is wide; they are employed for feature selection and generation, classification processes, and various image-processing tasks.

Here a selection [43] of classic metaheuristics and simpler search algorithms is employed and evaluated as parameter optimizers in the investigated framework. More specifically, classic variants of two well-known real-valued population-based optimizers are used, namely Differential Evolution (DE) [44] and Particle Swarm Optimization (PSO) [45]. The "DE/rand/1/bin" [44] variant of DE is used with meta-parameters empirically tuned (30 Agents, $\mathrm{F}=0.75, \mathrm{CR}=0.3$ ). Similarly, for PSO (30 Agents, Inertia Weight $=0.7$, Best own weight $=1.5$, Best weight $=1.5)$. A Hill Climber $(\mathrm{HC})$ is also employed $(\mathrm{D}=30)$, along with random sampling $(\mathrm{RND})$.

\subsubsection{Empirical Discrepancy Metrics}

Empirical discrepancy metrics [46] constitute a family of measures used to evaluate the quality of image segmentation. They are supervised, as they need a reference or ground truth to compare generated results with produced results. Notions of geometry, overlapping area, boundary offsets, and content are commonly encoded in such metrics, either via producing a singular result or as separate results (multi-objective frameworks). Analytical or unsupervised measures may also be considered [47]. A selection of empirical discrepancy metrics is employed here, namely the Reference Weighted Jaccard (RWJ) [6], Reference Bounded Segments Booster (RBSB) [48], and the Partial and Directed Object-Level Consistency Error (PD_OCE) [6,49]. They are chiefly based on the concept of area overlap, with the ability to measure notions of over- and under-segmentation. These metrics are summarized in Table 4.1 ([6,48,49]) (set theory notation), with $\mathrm{R}$ and $\mathrm{S}$ denoting a reference and generated (S) segment respectively. $\mathrm{I}$ is an iterator running through all generated segments (S) intersecting R. Their formulations are substantially different (some more precise, some more general), allowing for a varied interpretation of results and also for creating variation in search landscape characteristics (when employed to direct a search process). The optimal result for an evaluation is zero. 
Table 4.1. The three empirical discrepancy metrics employed to measure the quality of generated segments against the provided reference segments.

\begin{tabular}{ccc}
\hline Metric & Formulation & Reference \\
\hline RWJ & $1-\sum_{i=1}^{n} \frac{\left|R \cap S_{i}\right|}{\left|R \cup S_{i}\right|} \times \frac{\left|R \cap S_{i}\right|}{|R|}$ & {$[6]$} \\
RBSB & $\frac{|R \cup S|-|R \cap S|}{|R|}$ & {$[48]$} \\
PD_OCE & $1-\sum_{i=1}^{n} \frac{\left|R \cap S_{i}\right|}{\left|R \cup S_{i}\right|} \times \frac{\left|S_{i}\right|}{\sum_{j=1}^{n}\left|S_{j}\right|}$ & {$[6,49]$} \\
\hline
\end{tabular}

\subsubsection{Sample Supervised Segment Generation}

Sample supervised segment generation comprises a general image processing approach where an image segmentation task is cast as a search or optimization problem. A segmentation algorithm is defined, with its controlling aspects cast as variables or parameters forming a multidimensional search problem. Such an approach was initially proposed to simply tune the free parameters of a given segmentation algorithm for a given image analysis task [4]. Research into this general approach has been presented in various image analysis disciplines e.g., [4,7,50], including remote sensing [5,6,51]. Generally an appropriate level of a hierarchical segmentation algorithm is sought for a given image element type (e.g., specific buildings). In general, metaheuristics are employed as optimizers and empirical discrepancy metrics to drive the search process. Alternatively said, empirical discrepancy metrics may define the search landscape. Note that sample supervised segment generation is a specific implementation of the more general notion of image analysis via efficient search. See [52] for a primer. The method presented in Section 4.3 follows this general approach of sample supervised segment generation, where more detail may be found.

Various aspects of such an approach have been examined, including the applicability of various search methods e.g., [6,51], metric behavior [53,54] and the performance of domain-specific segmentation algorithms in this context [6,50]. Generalizability to unseen data, sampling requirements, method extensions, and method integration are open topics $[6,54,55]$. Note that parameters may also define construction processes of lower-level building blocks for image analysis, with mathematical morphology and genetic programming well suited to such designs [56,57].

\subsection{Method}

\subsubsection{Method Details}

A variant of sample supervised segment generation is presented [3] (conference paper), incorporating mapping functions for data adaptation and additional attributes for constraining segment growth. Figure 4.3 illustrates the architecture of the method, which is based on the general architecture of sample supervised segment generation [4]. The main distinguishing aspect of this variant is the three interacting parameter constituents handled by the given optimizer.

Initially a selection (5-50) of samples or reference segments is provided for the method, obtainable by various means (manual, semi-automated, or automated). A pre-processing phase conducted exclusively to save computing costs extracts image subsets centered over the reference segments. 
This may impact results in scenarios with non-unique segmentation algorithms. CC as used here generates unique results. The image subsets and accompanying reference segments are given as input to the optimization loop (see Figure 4.3).

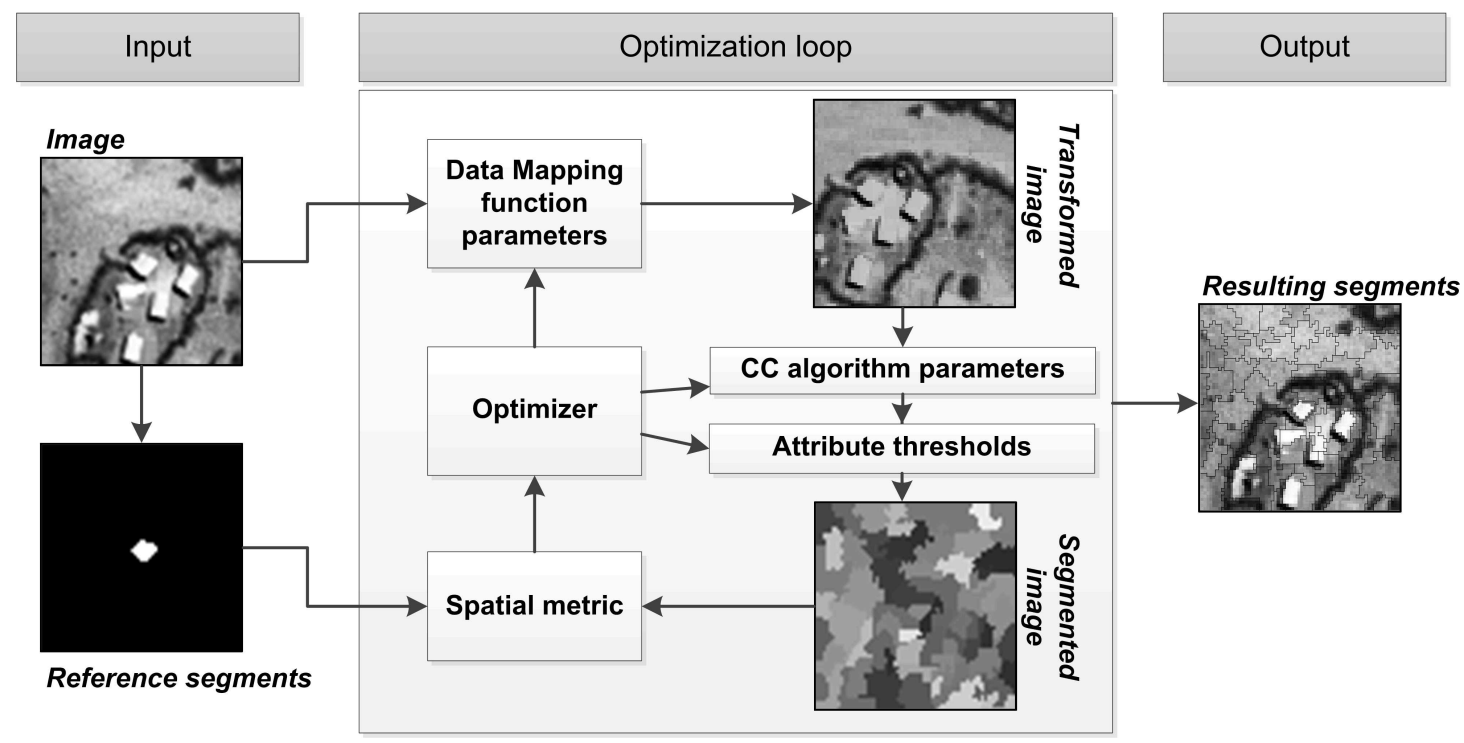

Figure 4.3. The architecture of the sample supervised segment generation method incorporating data mapping functions and attribute thresholding [3]. IEEEC 2013. Reprinted, with permission, from [3].

During the optimization loop phase, an optimizer (e.g., DE) traverses a parameter set over a certain number of iterations, which may control various image-processing functions on the image subsets. Three constituent parameter sets are defined in this method. Firstly, the controlling parameters of a given data mapping function transform or map the image subsets to a new domain. A few mapping functions are investigated with this method, detailed in Section 4.3.2. The transformed image subsets are subjected to the CC segmentation algorithm, with the optimizer providing the values for the local and global range parameters. This two-dimensional parameter set is the second constituent. The third constituent is a selection of segment spectral and geometric attributes, with the generated values defining attribute thresholds preventing segment growth within the attribute-enhanced CC framework. The image subsets are thus transformed and segmented, with the three parameter constituents controlling the characteristics of the generated segments.

The generated segments are evaluated (averaged) against the provided reference segments via a given empirical discrepancy metric. The RWJ, PD_OCE, and RBSB metrics are used here. The metric score is given to the optimizer as feedback of the performance of the given parameter set (all three constituents). The optimizer uses the information on quality to direct its search process for the next iteration of the optimization loop. Various termination criteria are possible for the optimization loop; here it is simply terminated after a certain number of iterations.

Finally after the optimization loop terminates, the best performing parameter set is given as output. The entire image may subsequently be segmented with the same image processing as employed in the optimization loop, with the image processing operators set with the output parameter set. Note that 
data mapping is conducted for segment generation and the byproduct is not used in further image processing. Also, the search process is used only during the training phase.

Note that there exists an optimal achievable result, within all the parameter value combinations. This result may not be perfect, i.e., not match the provided reference segments exactly, but is as good as it can get. In practice an exact match is highly unlikely. Also note that a search method may fail in finding the optimal achievable result. Multiple optimal achievable results are also possible, e.g., multiple parameter sets delivering the same optimal achievable metric score.

The three parameter constituents of the method are evaluated for their relative usefulness in a general setting. The presented method may function in four distinct ways. Firstly, the method may simply optimize the two parameters of CC, without any extra data mapping of attribute thresholding processes. This variant is simply called CC. Secondly, additional constraining attributes may be introduced, with this variant called $\mathrm{CC}+\mathrm{Attr}$ (Attributes). Alternatively, CC may function with an additional mapping function, called CC + Map (Mapping function). As depicted in Figure 4.3, the full method entails tuning all three constituents, called CC + Attr + Map. Figure 4.4 illustrates an example of an 11-dimensional parameter set traversed by an optimizer in the full formulation of the method $(\mathrm{CC}+\mathrm{Attr}+\mathrm{Map})$. Constituents are detailed in the next sections.

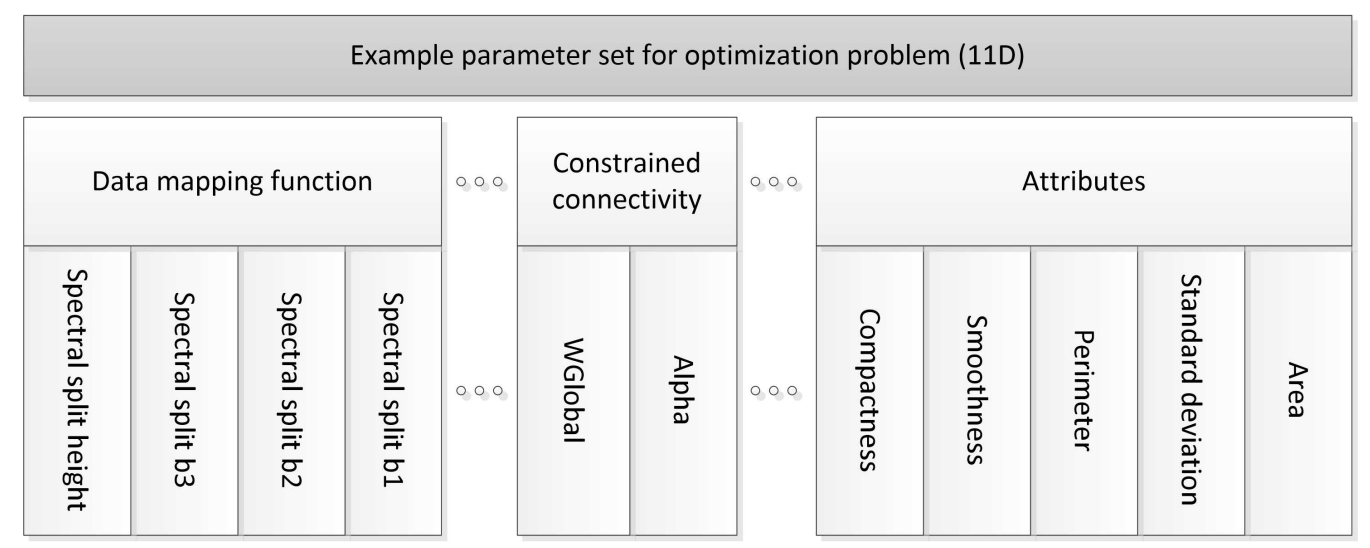

Figure 4.4. An example of an 11-dimensional parameter set traversed by the CC + Map + Attr full method variant. Example parameters within each constituent are written vertically.

In the situation of discrete parameter quantization (byte and short in this implementation), Figure 4.4 illustrates an optimization problem with more than $3 \times 10^{23}$ unique parameter combinations. The nature of the image processing (which these parameters control) dictates the difficulty of the search problem. An efficient search method may only search a fraction of such a space to obtain an optimal or near optimal result. This is strongly dependent on the "searchability" of the resultant search surface [4]. The validity of the proposed method as a valid optimization problem is also investigated, with a selection of optimizers experimentally evaluated. Details of the mapping functions used and their attribute constituents are briefly given.

\subsubsection{Mapping Functions}

In the generic formulation of $\mathrm{CC}$, basic dissimilarity is defined by the spectral difference between two pixels. Alternative definitions of connectivity may be considered as detailed in Section 4.2.2. In 
this work an indirect approach is followed, where spectral dissimilarity is changed externally via a data mapping function. Adding mapping functions shows promise for increasing the optimal achievable segmentation quality in similar frameworks in the context of remote sensing image analysis [6]. Some works have been proposed to reduce the influence of gradient zones based on external processing $[8,13]$ in the context of CC. Three mapping functions are tested [6], briefly described below. Figure 4.5 illustrates example results by running these functions. In this work three image bands (eight-bit quantization) are assumed.

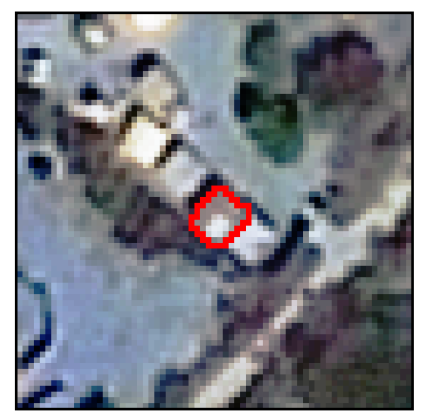

(a)

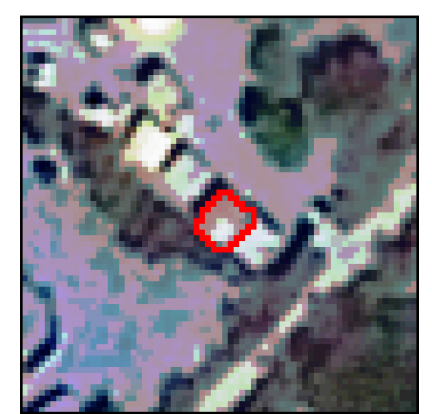

(b)

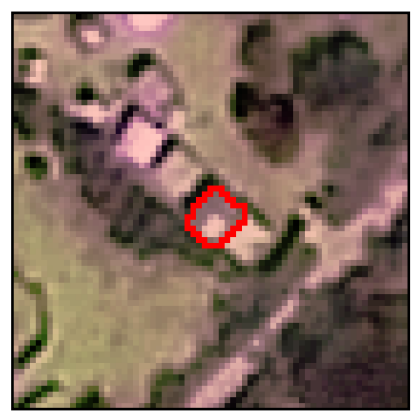

(c)

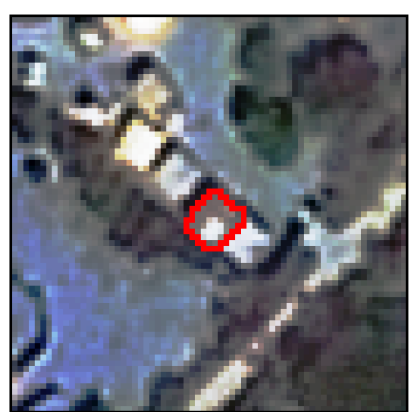

(d)

Figure 4.5. Example output of the three used mapping functions on an arbitrary test image (a). Parameters were assigned random values. The red polyline denotes an example element of interest; (b) shows output of the SS function (note the creation of sharp gradients); (c) shows output from the transformation matrix, while (d) shows the output from the GT function. Note the non-linear stretch of the output from the GT function.

\subsubsection{Spectral Split}

Spectral Split (SS) [6] is a simplistic function able to create artificial edges in gradient zones, based on the tuning of two parameters. It is given by:

$$
f(x)=x-(x-p)+\operatorname{sign}(x-p) \times h \text {, if abs }(x-p) \leq h, \text { else } f(x)=x
$$

where $p$ defines a position in the spectral domain (band specific) and $h$ defines the magnitude of spectral change around the given spectral position $(p) ; x$ remains unchanged if not falling within the required bounds $(h)$. The function is useful in scenarios where the boundaries of elements of interest are not distinct or span multiple pixels. Sign extracts the sign of the number, with zero given a positive sign.

\subsubsection{Transformation Matrix}

A transformation matrix (LIN) with three image bands is used as a mapping function. Considering three input bands, nine parameters $(\mathrm{a}-\mathrm{i})$ define the transformation matrix. A pixel (n1-n3/b1-b3) is defined by the point matrix:

$$
\left[\begin{array}{l}
n 1 \\
n 2 \\
n 3
\end{array}\right]=\left[\begin{array}{lll}
a & b & c \\
d & e & f \\
g & h & i
\end{array}\right]\left[\begin{array}{l}
b 1 \\
b 2 \\
b 3
\end{array}\right]
$$

The range of the parameters is set to $[-0.2,1]$, allowing for the enhancement of negative band correlations if present and if found useful by a search process. 


\subsubsection{Genetic Transform}

A function, developed as a parameterized low-level image processing method for image enhancement [58], consists of four parts conducting data stretching (parameters p1-p5) and weighs their contribution based on additional weighting parameters (parameters p6-p10). For convenience the function is called Genetic Transform (GT). It is written as:

$$
\begin{gathered}
f(x)=\frac{\log \left(1+e^{p 1-1} \times x\right)}{p 1} \times p 7+\frac{(1+p 2)^{x}-1}{p 2} \times p 8+\frac{1}{1+\left(\frac{(1-x)}{p 3}\right)^{p 4}} \times p 9 \\
+\frac{1}{\left(\frac{1}{x}+p 6-1\right)^{p 5}} \times p 10
\end{gathered}
$$

As with the SS function, the GT function may assist in sharpening boundaries in image elements. In the case of GT, this is achieved via parameterized non-linear data stretching.

\subsubsection{Attributes}

Six attributes are defined for consideration as additional thresholding criteria in the presented method. Table 4.2 summarizes the used attributes and their given ranges. Area, variance, and perimeter are simple attributes, conjectured to add some benefit in many instances. The gray level difference histogram $(\mathrm{CH})$, defined here with five bins $(\mathrm{CH} 1-\mathrm{CH} 5)$, counts the number of instances of gray level differences in a segment falling within the given bin constraint. Here a 4-connected pixel design is considered. The bin ranges are given in Table 4.2. For example, if a segment contains only two pixels and the spectral difference between them is 7 (intensity difference), the second bin ( $\mathrm{CH} 2$ ) will have a value of 1 . The other bins $(\mathrm{CH} 1, \mathrm{CH} 3, \mathrm{CH} 4, \mathrm{CH} 5)$ will have a value of zero.

Note the intrinsic link between the gray level difference histogram bins and the local and global range parameters of CC. Small values of the local and global range parameters may lead to empty $\mathrm{CH}$ bins. All attributes are computed incrementally during the execution of CC (if attributes are used).

Table 4.2. Implemented attributes for consideration in the context of $\mathrm{CC}$ segmentation, specifically in the CC + Attr and CC + Attr + Map method variants.

\begin{tabular}{cll}
\hline Attribute & Range & Description \\
\hline Area & {$[0 . .500]$} & Segment area measured in number of pixels \\
Standard Deviation & {$[0 . .255]$} & Segment spectral standard deviation \\
Perimeter & {$[0 . .500]$} & Number of pixel edges forming the perimeter \\
Smoothness (SMT) & {$[0 . .30]$} & Perimeter/sqrt(area) \\
Compactness (CMP) & {$[0 . .30]$} & Perimeter/bounding box edge length \\
Gray level difference Histogram, & {$[0 . .500]$ Bins: CH1:[0..5], CH2:(5..10], } & Number of edge weights falling within \\
five bins (CH1-5) & $\mathrm{CH}:(10 . .15], \mathrm{CH} 4:(15 . .20], \mathrm{CH}:(20 . .255]$ & specified bins. Five bins are defined. \\
\hline
\end{tabular}

Figure 4.6 illustrates the same image subset as shown in Figure 4.2, with the local range parameter set to 50 and the global range parameter to 200, but with additional constraining attributes added for illustration. Specifically, Figure 4.6a shows the addition of the area attribute set to 800 in this instance. Compare with Figure 4.2d, where the local and global range parameters are the same. Figure 4.6b shows the addition of the $\mathrm{CH} 1 \mathrm{bin}$, set to 300 . Intuitively the impact of the constraints may be 
interpreted as the largest segments possible (hierarchical) under a local range of 50 (or lower), still satisfying a global range of 200 in addition to an area criterion of 800 (Figure 4.6a) or a CH1 criterion of 300 in the case of Figure 4.6b.

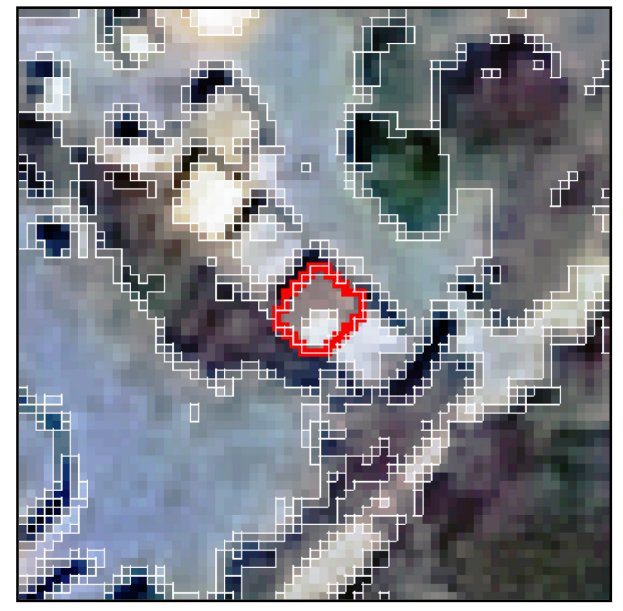

(a)

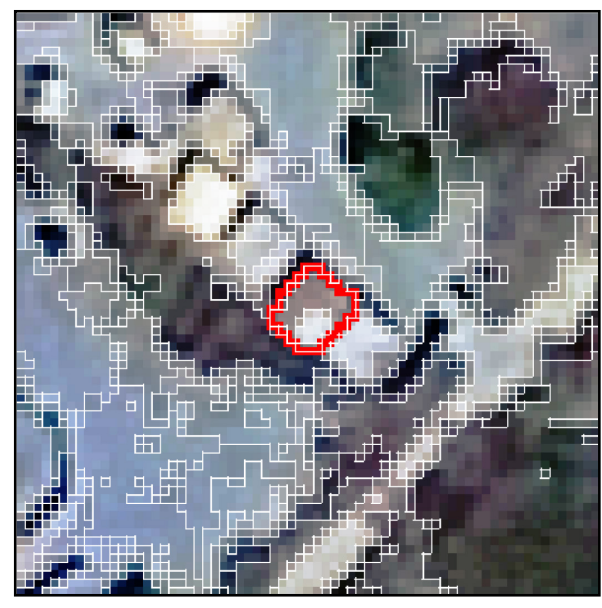

(b)

Figure 4.6. An image subset segmented with the local range parameter set to 50 and the global range parameter set to 200. Additional constraining attributes are introduced, specifically area, with a value of 800 (a) and CH1 (b) with a value of 300 .

\subsection{Data}

Three image analysis tasks or problems were defined for evaluating the performance of the method variants (subset of data used in [6]). The aim was to segment structure subtypes accurately. The resulting segmentation, of maximal achievable quality, may be used in further processing methodologies. For each dataset a characteristic structure type was identified and defined as the element of interest.

Figure 4.7 illustrates subsets of the data and enlargements over elements of interest. Site 1, titled Bokolmanyo, depicts a refugee camp with easily identifiable tents as the elements of interest. In practice this problem could be approached with a simple single-layer segmentation and classification method. Site 2 (Jowhaar) and Site 3 (Hagadera) depict more difficult image analysis problems. The Jowhaar problem entails segmenting metal-roofed structures, with variation in roof geometry and reflectance angles ensuring a more challenging problem. Similarly the Hagadera problem entails segmenting metal-roofed structures, but with much larger variations in reflectance and geometry. A range of problem difficulties is thus presented, with a comparative analysis of segment quality the focus, rather than the final segmentation accuracies.

For each problem a number of reference elements were digitized and used as input to the presented method variants. Table 4.3 details some metadata of the three datasets used. All imagery consists of three bands, fully preprocessed and standardized to 8-bit quantization. The number of reference segments used is also given. Two-fold cross-validation is performed in experimentation, thus a random selection of half of the reference segments is used to drive the search process. The training and testing sets are constantly changed between experimental runs. Preliminary experimentation with varying sampling sizes was conducted to find stable results under cross-validated conditions. At least 20 runs 
per experiment are also advocated (detailed in the next section). This coupled with two-fold crossvalidation in each run ensures a measure of generalizability in results. Note that study area size is not an important consideration. Subset images are generated, centered on the provided reference segments (e.g., Figures 4.2,4.5,4.6).

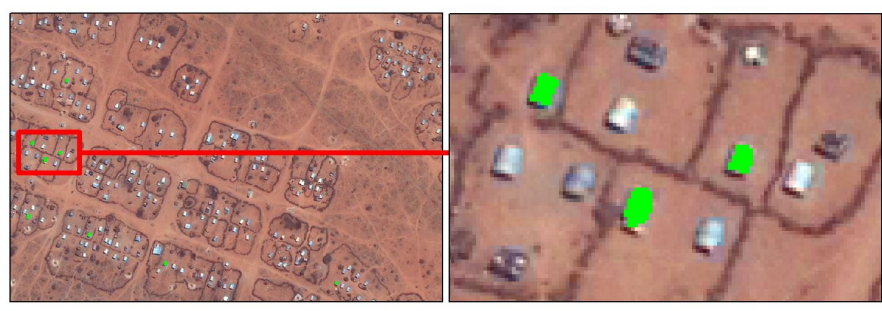

(a)

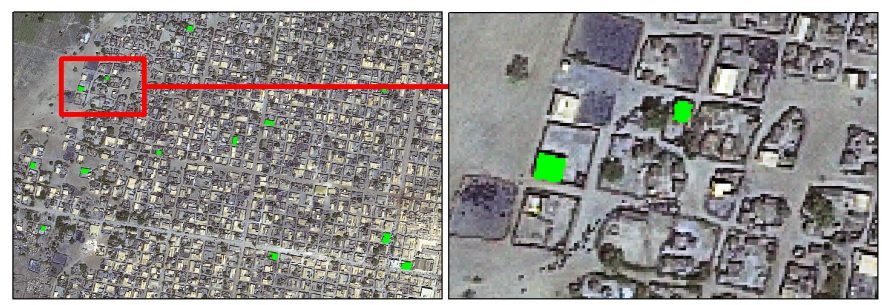

(b)

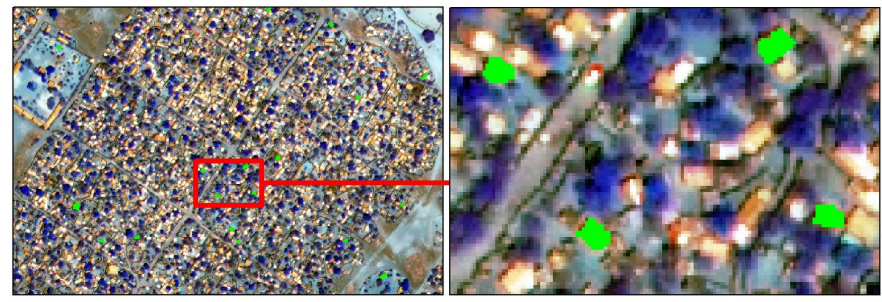

(c)

Figure 4.7. The three image analysis tasks defined for evaluating the method variants, namely, thematically correctly segmenting tents in the Bokolmanyo problem (a) and metalroofed structures in the Jowhaar (b) and Hagadera (c) problems.

Table 4.3. The datasets, with accompanying metadata, used for evaluating the method variants (adapted from [6]).

\begin{tabular}{ccccccc}
\hline \multirow{2}{*}{ Test Site } & $\begin{array}{c}\text { Target } \\
\text { Elements }\end{array}$ & Sensor & $\begin{array}{c}\text { Spatial } \\
\text { Resolution }\end{array}$ & $\begin{array}{c}\text { Reference } \\
\text { Segments }\end{array}$ & Channels & Date Captured \\
\hline Bokolmanyo $^{1}$ & Tents & GeoEye-1 & $0.5 \mathrm{~m}$ & 28 & $1,2,3$ & $24 / 8 / 2011$ \\
Jowhaar $^{1}$ & Structures & GeoEye-1 & $0.5 \mathrm{~m}$ & 40 & $1,2,3$ & $26 / 02 / 2011$ \\
Hagadera $^{2}$ & Structures & WorldView-2 & $0.5 \mathrm{~m}$ & 38 & $4,6,3$ & $07 / 10 / 2010$ \\
\hline
\end{tabular}

${ }^{1}$ GeoEye, Inc. ${ }^{\odot} 2011$, provided by e-GEOS S.p.A., under GSC-DA, all rights reserved.; ${ }^{2}$ DigitalGlobe, Inc. ${ }^{\odot}$ 2010, provided by EUSI under EC/ESA/GSC-DA, all rights reserved.

\subsection{Experimental Evaluation}

The presented method is firstly analyzed to verify that it constitutes a multidimensional search problem with parameter interdependencies measured among all constitute components (Section 4.5.1). A range of common metaheuristics is then tested on the method, evaluating the merits of using more 
complex search methods compared with simpler search strategies (Section 4.5.2). Finally an extensive relative comparison is conducted (Section 4.5.3) on the four method variants under a variety of metric and problem conditions. The merits of the variants are highlighted via measuring computing costs and generating a statistical ranking under cross-validated conditions.

\subsubsection{Parameter Interdependencies}

The parameters of the $\mathrm{CC}+$ Attr + Map method variant, specifically using the GT function for mapping, are profiled for interdependencies using a statistical parameter interdependency test. Without interdependencies among constituents, such a multidimensional problem may be decomposed into smaller, independently solvable problems. The test, able to profile the frequency of parameter interdependencies [59], is briefly described.

A given parameter $x_{i}$ is affected by another $x_{j}$ if a change in the ordering of solution finesses is observed by independently varying values for $x_{i}$ and $x_{j}$ in arbitrary full parameter sets $(a, b)$. Formally, $x_{i}$ is affected by $x_{j}$ if

$$
\begin{aligned}
f(a) \leq & f(b) \& f\left(a^{\prime}\right)>f\left(b^{\prime}\right) \text { with } \\
a & =\left(\ldots, x_{-} i, \ldots, x_{-} j, \ldots\right) \\
b & =\left(\ldots, x_{-} i^{\prime}, \ldots, x_{-} j, \ldots\right) \\
a^{\prime} & =\left(\ldots, x_{-} i, \ldots, x_{-} j^{\prime}, \ldots\right) \\
b^{\prime} & =\left(\ldots, x_{-} i^{\prime}, \ldots, x_{-} j^{\prime}, \ldots\right)
\end{aligned}
$$

where the function $\mathrm{f}$ is the RWJ measure in this implementation.

This test may be repeated multiple times to generate an indication of the frequency of parameter interaction. A table may be generated, with the parameters labeled in the first column denoted as being affected by the parameters listed in the first row, if a value above zero is generated.

The parameter interdependency test is repeated 100 times for each parameter pair, using the $\mathrm{CC}+$ Attr + Map method variant for all three problems. The RWJ metric was used to judge a change in segment quality. Note that the metric can measure notions of over- and under-segmentation. Tables 4.4-6 report the number of affected cases for all parameters over the allocated 100 runs. For each problem having differing characteristics, both the parameters of the $\mathrm{CC}$ algorithm are shown with a random selection of parameters investigated for the GT mapping function and attribute thresholds. A method constituent (vertically listed "Mapping function", "CC parameters" and "Attributes") will be considered unaffected by another constituent (horizontally listed) if all values within the given subdivision are zero.

In all three problems, all parameter constituents are affected by all other constituents. The degree of interaction ranges from frequent, e.g., in the case of CC parameters and attribute thresholds affected by mapping function parameters, to very infrequent, such as in the case of the $\mathrm{CC}$ parameters affected by attribute thresholds. Generally, the investigated mapping function parameter affects other parameters most frequently. Interaction is present in all cases. This validates the presented method as a singular optimization problem. Note that the magnitude of the variation in solution quality is not recorded in these tests. Relative solution qualities are investigated in Section 4.5.3. Interestingly, note that the global range parameter is commonly affected more by the local range parameter (than vice versa), 
even though modifying the local range parameter beyond the value of the global range parameter has no effect [10].

Table 4.4. Interdependency test of the method constituents for the Bokolmanyo problem. Note that all constituents affect one another. The mapping function affects all parameters most frequently.

\begin{tabular}{lc|ccccccccc}
\hline \multirow{2}{*}{ Bokolmanyo } & \multicolumn{3}{c}{ Mapping Function } & \multicolumn{2}{c}{ CC } & \multicolumn{3}{c}{ Attributes } \\
\cline { 3 - 10 } & & GT1 & GT2 & GT10 & Local & Global & Area & Std & CH2 \\
\hline \multirow{2}{*}{ Mapping } & GT1 & & 15 & 19 & 2 & 0 & 3 & 0 & 2 \\
function & GT2 & 36 & & 29 & 3 & 0 & 2 & 3 & 2 \\
& GT10 & 38 & 12 & & 4 & 0 & 2 & 1 & 3 \\
\hline \multirow{2}{*}{ CC } & Local & 6 & 13 & 11 & & 1 & 2 & 0 & 1 \\
& Global & 31 & 15 & 24 & 12 & & 6 & 0 & 2 \\
\hline \multirow{3}{*}{ Attributes } & Area & 19 & 28 & 22 & 2 & 1 & & 0 & 3 \\
& Std & 21 & 25 & 32 & 1 & 1 & 11 & & 2 \\
& CH2 & 13 & 11 & 9 & 1 & 0 & 1 & 0 & \\
\hline
\end{tabular}

Table 4.5. Interdependency test of the method constituents for the Jowhaar problem.

\begin{tabular}{lc|ccc|ccc|ccc}
\hline \multicolumn{2}{c}{ Jowhaar } & \multicolumn{3}{c}{ Mapping Function } & \multicolumn{3}{c}{ CC } & \multicolumn{3}{c}{ Attributes } \\
\cline { 3 - 10 } & & GT3 & GT4 & GT9 & Local & Global & Perim & Smooth & CH1 \\
\hline \multirow{2}{*}{$\begin{array}{c}\text { Mapping } \\
\text { function }\end{array}$} & GT3 & & 33 & 20 & 3 & 0 & 1 & 0 & 1 \\
& GT4 & 8 & & 9 & 4 & 0 & 0 & 1 & 0 \\
& GT9 & 19 & 34 & & 4 & 2 & 1 & 1 & 1 \\
\hline \multirow{2}{*}{ CC } & Local & 13 & 16 & 11 & & 7 & 0 & 2 & 0 \\
& Global & 17 & 18 & 20 & 12 & & 10 & 0 & 4 \\
\hline \multirow{3}{*}{ Attributes } & Perm & 20 & 24 & 18 & 2 & 3 & & 1 & 6 \\
& Smooth & 8 & 3 & 2 & 1 & 0 & 0 & & 0 \\
\hline
\end{tabular}

Table 4.6. Interdependency test of the method constituents for the Hagadera problem.

\begin{tabular}{lc|ccc|ccc|ccc}
\hline \multirow{2}{*}{ Hagadera } & \multicolumn{3}{c}{ Mapping Function } & \multicolumn{2}{c}{ CC } & \multicolumn{3}{c}{ Attributes } \\
\cline { 3 - 11 } & & GT6 & GT7 & GT8 & Local & Global & CH3 & CH4 & CH5 \\
\hline \multirow{2}{*}{$\begin{array}{l}\text { Mapping } \\
\text { function }\end{array}$} & GT6 & & 27 & 15 & 3 & 3 & 3 & 1 & 3 \\
& GT7 & 29 & & 25 & 5 & 1 & 4 & 1 & 2 \\
& GT8 & 23 & 33 & & 7 & 4 & 1 & 0 & 1 \\
\hline \multirow{2}{*}{ CC } & Local & 10 & 7 & 9 & & 4 & 3 & 1 & 4 \\
& Global & 27 & 13 & 20 & 6 & & 4 & 0 & 0 \\
\hline \multirow{3}{*}{ Attributes } & CH3 & 6 & 3 & 3 & 0 & 0 & & 1 & 4 \\
& CH4 & 4 & 3 & 2 & 0 & 0 & 0 & & 0 \\
& CH5 & 3 & 2 & 0 & 0 & 0 & 1 & 0 & \\
\hline
\end{tabular}

For illustrative purposes, Figure 4.8 shows exhaustive fitness calculations (RWJ metric) for arbitrary two-dimensional slices of the parameter space (also called the search surface). Figure 4.8a shows the interaction of the local range parameter of the $\mathrm{CC}$ algorithm interacting with the $\mathrm{B} 1$ parameter of the spectral split mapping function. Note two local optima. All other parameters were 
given initial random values and were kept constant during the generation of the search surface. Figure $4.8 \mathrm{~b}$ illustrates similarly, with a simpler interaction (single optimal) between thresholds of the $\mathrm{CH} 1$ attribute and the local range parameter of CC. Note that these figures are illustrative of parameter interactions and may be vastly different (more complex/less complex) under different external parameter conditions.

\subsubsection{Search Surface Complexity}

In Section 4.5.1, it was shown that parameter interactions exist in the presented method. Some interactions are frequent in the case of selected constituents. Here we investigate the applicability [39] of a range of search methods to traverse the search surfaces of the four method variants. Intuitively the $\mathrm{CC}$ variant of the method, with a relatively simple interaction between the local and global range parameters, would not be a difficult search problem. Simple parameter tuning would be feasible in such a scenario using the $\mathrm{CC}$ variant of the method, or a simple grid search or random parameter search.

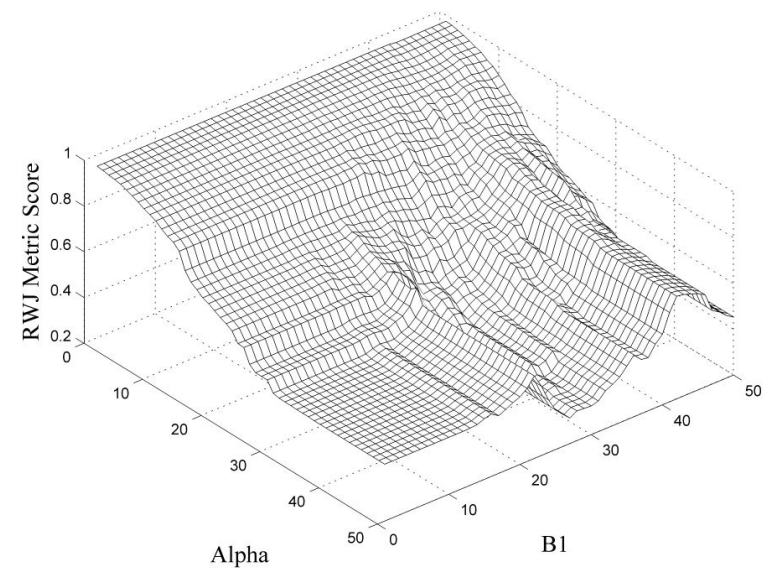

(a)

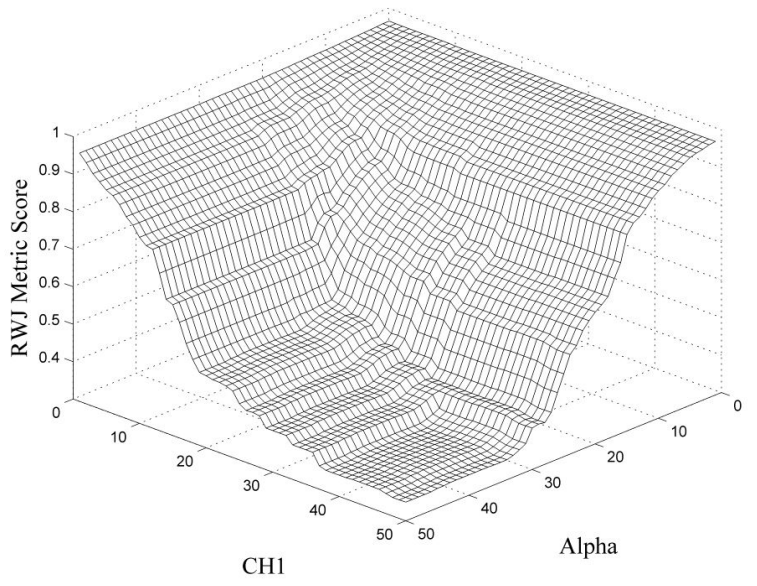

(b)

Figure 4.8. Two-dimensional parameter plots, or search surfaces, demonstrating parameter interactions between method constituents: (a) illustrates the interaction of the alpha parameter from the $\mathrm{CC}$ constituent and that of a mapping function parameter, while (b) shows the interaction of alpha with the $\mathrm{CH} 1$ attribute.

The four method variants are run on the Bokolmanyo problem (GT mapping), conjectured to exhibit the simplest search surfaces. Four search methods, namely random search (RND), HillClimber (HC), standard particle swarm optimization (PSO), and a standard variant of Differential Evolution (DE) are investigated (Section 4.2.3). The RWJ metric is used to judge segment quality. The search process is granted 2000 iterations. Thus, although the tested search methods have vastly different mechanisms (single or population-based, stochastic or deterministic), they are evaluated based on an equal computing budget. Each experiment is repeated 20 times. Averages over the 20 runs are quoted, with the standard deviations also given. The CC method variant has a two-dimensional parameter domain, 
the $\mathrm{CC}+$ Attr variant 12 dimensions, $\mathrm{CC}+$ Map also 12 and the $\mathrm{CC}+\mathrm{Attr}+$ Map method variant 22. Cross-validation was not performed, as search method progress and feasibility were evaluated.

Table 4.7 lists the optimal achieved metric scores (RWJ) given 2000 search method iterations. The shaded cells indicate the search methods achieving the best scores for each method variant. Various ties in optimal results among the search methods are noted. Firstly, on examining the CC method variant, as expected, no benefit is seen from using more complex search methods. Note that even on this simple search surface, the HC method could not find the global optimal routinely. Similarly, adding attribute thresholding as additional parameters $(\mathrm{CC}+\mathrm{Attr})$ gave similar results across the different search methods. Again, HC performed worse than the other methods. In these two method variants, RND, PSO, and DE routinely generated the optimal results. Note that initialization of the parameters in the search processes was random (as opposed to, for example, distributed hypercube sampling). Interestingly, none of the search methods was able to find optimal values on the edge of the search domain when attributes were introduced (owing to random initialization).

Table 4.7. Performance of the four search methods on the four method variants. In the simpler CC method variants ( $\mathrm{CC}$ and $\mathrm{CC}+\mathrm{Attr})$, no benefit is noted from using more advanced search methods. In the case of the higher dimensional method variants $(\mathrm{CC}+$ Map and CC + Attr + Map), using an advanced search method becomes necessary.

\begin{tabular}{ccccc}
\hline & CC & CC + Attr & CC + Map & CC + Attr + Map \\
\hline RND & $0.429 \pm 0.000$ & $0.448 \pm 0.000$ & $0.186 \pm 0.012$ & $0.193 \pm 0.015$ \\
HC & $0.442 \pm 0.009$ & $0.535 \pm 0.144$ & $0.507 \pm 0.083$ & $0.538 \pm 0.159$ \\
PSO & $0.429 \pm 0.000$ & $0.448 \pm 0.000$ & $0.167 \pm 0.012$ & $0.163 \pm 0.008$ \\
DE & $0.429 \pm 0.000$ & $0.448 \pm 0.000$ & $0.161 \pm 0.003$ & $0.163 \pm 0.003$ \\
\hline
\end{tabular}

Considering the CC + Map and CC + Attr + Map variants of the method, the more complex search methods (PSO, DE) performed substantially better (statistically significantly different, Student's $t$-test with a 95\% confidence interval) than the RND and especially the HC search method. A difference in 0.030 in the case of the RWJ metric when results approach their optimum is in a practical sense very noticeable. This suggests that under the higher dimensional problem conditions, with more complexities introduced by a mapping function, stochastic population-based search strategies (or others) are needed. Note the slight decrease in standard deviation in the most complex method variants. Also, as generally documented [60], the generic variant of DE performed slightly better than the generic variant of PSO. Further results are presented exclusively with the DE method.

Figure 4.9 shows the search progress profiles over the allocated 2000 iterations (averaged over 20 runs) for the CC (Figure 4.9a), CC + Attr (Figure 4.9b), CC + Map (Figure 4.9c), and CC + Attr + Map (Figure 4.9d) method variants. Note that in the simpler method variants (CC and CC + Attr), the optimal results are achieved within 100 method iterations. The more complex method variants $(\mathrm{CC}+\mathrm{Map}$ and $\mathrm{CC}+$ Attr + Map) need substantially more search iterations to achieve optimal or near-optimal results. Figure $4.9 \mathrm{c}, \mathrm{d}$ also shows that under the more complex problem formulations, PSO and DE provide better results relatively early on in the search process, suggesting their use even under constrained processing conditions. These plots reveal that method termination may be suggested at around 1000 iterations in these method formulations, or an alternative termination condition may be encoded based on derivatives observed between 500 and 1000 iterations. 


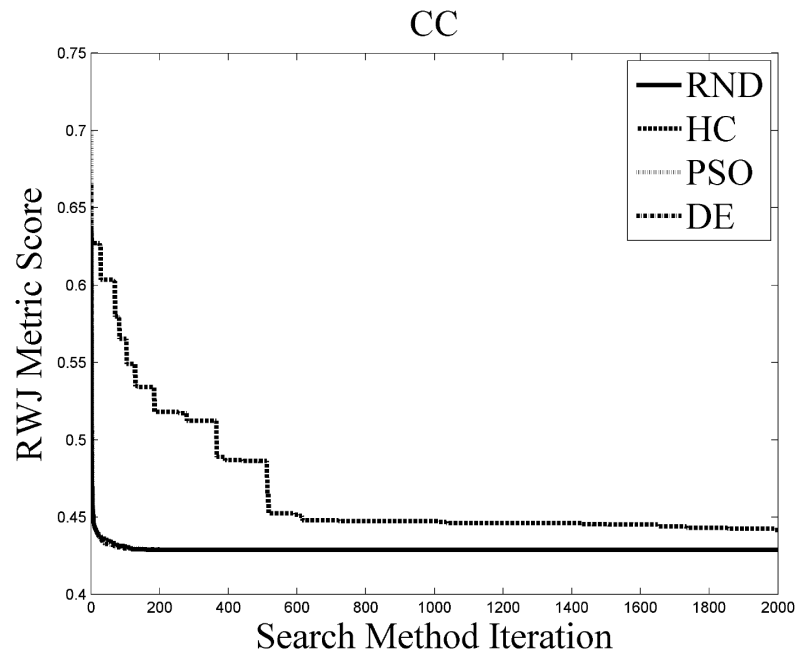

(a)

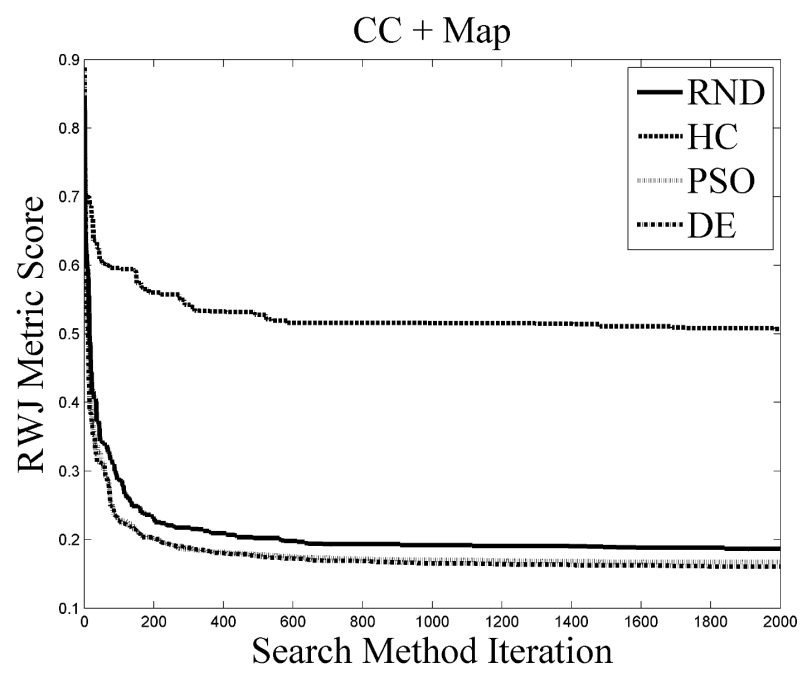

(c)

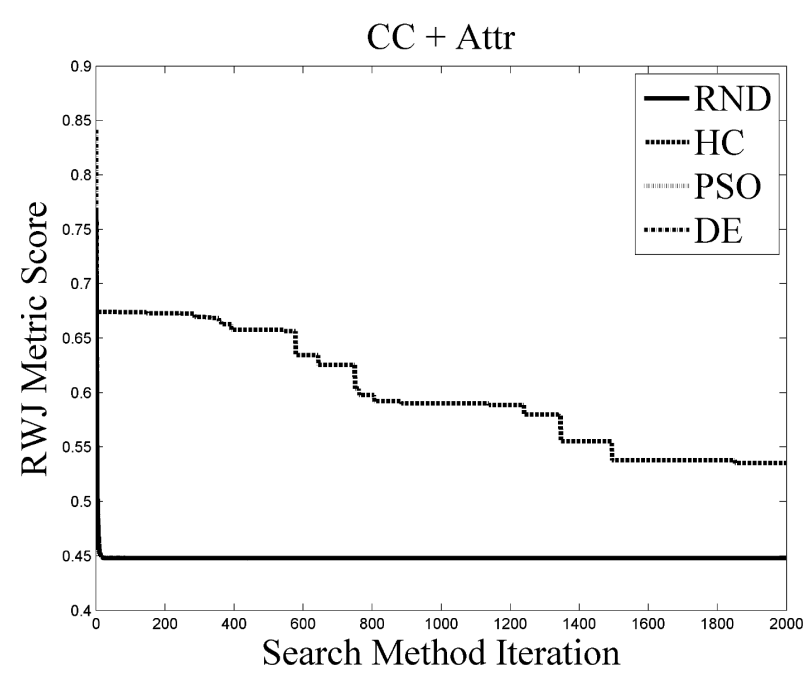

(b)

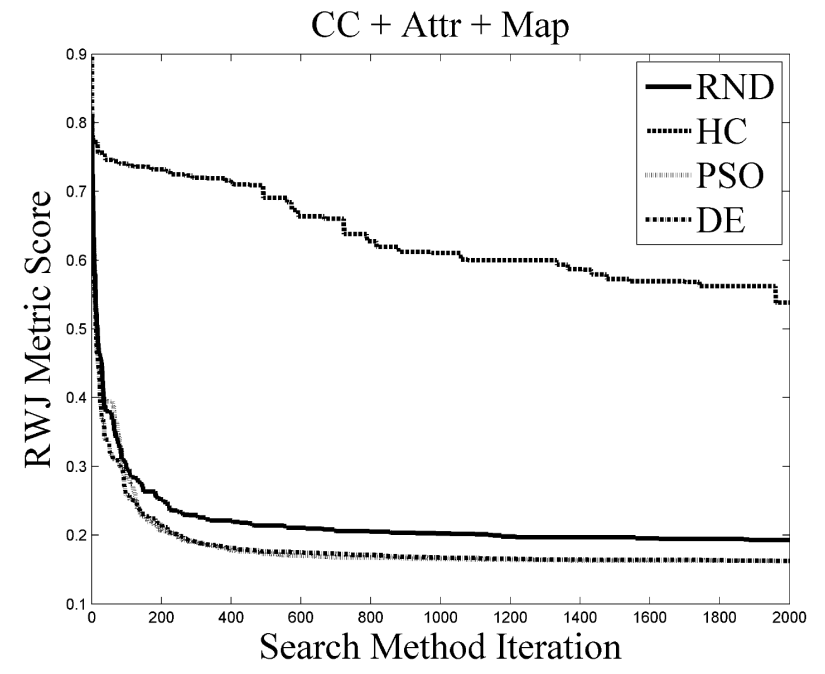

(d)

Figure 4.9. Search method profiles for the four method variants, namely CC (a), CC + Attr (b), CC + Map (c), and CC + Attr + Map (d). Note the increased performance of DE and PSO when considering the CC + Map and CC + Attr + Map method variants.

\subsubsection{Method Variant Performances}

The four presented method variants are evaluated, relative to one another, based on maximal achieved metric scores under cross-validated conditions. Profiling such relative performances in general may give an indication of the merits of the constituents in such a framework. Computing times are also contrasted, as well as convergence behavior, which are important considerations to reduce method processing times. For each problem (Bokolmanyo, Jowhaar, Hagadera), the four method variants are run using all three detailed empirical discrepancy metrics. Each experiment is repeated 20 times, with averages and standard deviations reported. For each site a random mapping function was selected (SS, LIN, or GT). In addition, the best results obtained during the 20 runs are also reported. Thus for each method variant, nine differentiated segmentation tasks (problem type, metric characteristic) are evaluated with over 50 million individual segment evaluations conducted.

Tables 4.8-10 list the achieved metric scores for the problems under different metric and method variant conditions. Note that method variants may be contrasted based on a given metric and not via 
different metric values. On examining Table 4.8, depicting the Bokolmanyo problem, it is clear that the more elaborate method variants employing a mapping function (LIN in this case) and a mapping function plus attributes generated superior results compared with the $\mathrm{CC}$ and $\mathrm{CC}+\mathrm{Attr}$ variants. Interestingly, under cross-validated conditions, the addition of constraining attributes $(\mathrm{CC}+\mathrm{Attr})$ created an overfitting scenario, resulting in worse performances compared with not employing constraining attributes.

The performances of $\mathrm{CC}+\mathrm{LIN}$ and $\mathrm{CC}+\mathrm{Attr}+\mathrm{LIN}$ are similar, with the given metric dictating the superior method. Under the RBSB metric condition the CC + LIN method variant displays extremely sporadic results. This suggests that the search surfaces generated under this condition contain numerous discontinuities, creating difficulties for the DE search method. This may be due to the formulation, or nature, of RBSB. It is reference segment centric. In contrast, considering the CC + Attr method variant, the RBSB metric proved robust and similar to the $\mathrm{CC}$ variant in terms of optimal results.

Table 4.8. Method performance on the Bokolmanyo problem. Note the improved results with the $\mathrm{CC}+\mathrm{LIN}$ and $\mathrm{CC}+\mathrm{Attr}+\mathrm{LIN}$ method variants under all metric conditions.

\begin{tabular}{cccccc}
\hline & & CC & CC + Attr & CC + LIN & CC + Attr + LIN \\
\hline \multirow{2}{*}{ RWJ } & Avg & $0.465 \pm 0.000$ & $0.520 \pm 0.035$ & $0.239 \pm 0.020$ & $0.235 \pm 0.026$ \\
& Min & 0.465 & 0.476 & 0.211 & 0.200 \\
\hline \multirow{2}{*}{ RBSB } & Avg & $0.299 \pm 0.000$ & $0.308 \pm 0.009$ & $0.262 \pm 0.235$ & $0.185 \pm 0.034$ \\
& Min & 0.299 & 0.301 & 0.136 & 0.144 \\
\hline \multirow{2}{*}{ PD_OCE } & Avg & $0.538 \pm 0.009$ & $0.556 \pm 0.030$ & $0.233 \pm 0.016$ & $0.244 \pm 0.026$ \\
& Min & 0.526 & 0.514 & 0.199 & 0.205 \\
\hline
\end{tabular}

The Jowhaar problem (Table 4.9) displays a slightly different general trend. Under all metric conditions the mapping function method variant $(\mathrm{CC}+\mathrm{SS})$ proved superior to both the attribute $(\mathrm{CC}+$ Attr) and combined mapping function and attribute $(\mathrm{CC}+\mathrm{Attr}+\mathrm{SS})$ method variants. Under crossvalidated conditions, no benefit was seen from employing attributes, commonly leading to worse results. Note that generally the absolute results were poorer compared with the easier Bokolmanyo problem.

Table 4.9. Method performance on the Jowhaar problem. The method variant employing a data mapping function $(\mathrm{CC}+\mathrm{SS})$ performed the best under all metric conditions.

\begin{tabular}{cccccc}
\hline & & CC & CC + Attr & CC + SS & CC + Attr + SS \\
\hline \multirow{2}{*}{ RWJ } & Avg & $0.551 \pm 0.003$ & $0.784 \pm 0.001$ & $0.411 \pm 0.009$ & $0.757 \pm 0.013$ \\
& Min & 0.548 & 0.783 & 0.392 & 0.739 \\
\hline \multirow{2}{*}{ RBSB } & Avg & $0.622 \pm 0.000$ & $0.652 \pm 0.003$ & $0.418 \pm 0.058$ & $0.616 \pm 0.023$ \\
& Min & 0.622 & 0.649 & 0.348 & 0.581 \\
\hline \multirow{2}{*}{ PD_OCE } & Avg & $0.684 \pm 0.002$ & $0.825 \pm 0.006$ & $0.549 \pm 0.032$ & $0.807 \pm 0.021$ \\
& Min & 0.683 & 0.816 & 0.506 & 0.769 \\
\hline
\end{tabular}

The Hagadera problem (Table 4.10), considered the most difficult problem, exhibits curious results not corroborating trends observed in the previous two problems. Under different metric conditions, the three method variants $(\mathrm{CC}+\mathrm{Attr}, \mathrm{CC}+\mathrm{GT}$, and $\mathrm{CC}+\mathrm{Attr}+\mathrm{GT})$ all achieved the top performance. 
$\mathrm{CC}+\mathrm{GT}$ was superior under the RWJ metric condition, $\mathrm{CC}+$ Attr under the RBSB condition, and CC + Attr + GT under the PD_OCE condition.

Table 4.10. Method performances on the Hagadera problem. The top performing method variant is metric dependent.

\begin{tabular}{cccccc}
\hline & & $\mathbf{C C}$ & $\mathbf{C C}+\mathbf{A t t r}$ & $\mathbf{C C}+\mathbf{G T}$ & $\mathbf{C C}+\mathbf{A t t r}+\mathbf{G T}$ \\
\hline \multirow{2}{*}{ RWJ } & Avg & $0.614 \pm 0.000$ & $0.631 \pm 0.008$ & $0.492 \pm 0.013$ & $0.509 \pm 0.012$ \\
& Min & 0.614 & 0.619 & 0.468 & 0.494 \\
\hline \multirow{2}{*}{ RBSB } & Avg & $0.737 \pm 0.000$ & $0.511 \pm 0.014$ & $1.633 \pm 1.061$ & $0.522 \pm 0.045$ \\
& Min & 0.737 & 0.486 & 0.526 & 0.460 \\
\hline \multirow{2}{*}{ PD_OCE } & Avg & $0.705 \pm 0.001$ & $0.684 \pm 0.005$ & $0.617 \pm 0.023$ & $0.616 \pm 0.028$ \\
& Min & 0.704 & 0.678 & 0.579 & 0.553 \\
\hline
\end{tabular}

Figure 4.10 shows some optimal results obtained for various problem runs depicted in Tables $4.8-$ 10. Each sub-figure shows a given reference segment, delineated with a red polyline. Resulting segments for the best performing parameter sets are shown with white polylines. The RWJ metric scores for the specific segment are also quoted. The given metric scores are specific to the red delineated reference segments shown (randomly chosen) and not the averaged and cross-validated results generated during experimentation. Figure $4.10 \mathrm{a}-\mathrm{c}$ shows local optimal results for the $\mathrm{CC}$ method variant. Figure 4.10d-f presents the results under the $\mathrm{CC}+$ Attr method variant, Figure 4.10g- $\mathrm{i}$ for the CC + Map method variant and Figure 4.10j-1 for the CC + Attr + Map variant. Note the same results generated for the Jowhaar problem under $\mathrm{CC}$ and $\mathrm{CC}+$ Attr method conditions (Figure $4.10 \mathrm{~b}, \mathrm{e})$, with constraining attributes not affecting segment quality over the given reference segment.

Generally, based on observing Tables 4.8-10, the introduction of mapping functions provides more robust improvements under more conditions compared with adding attributes. In some cases a combination of attributes and a mapping function proved most useful. Table 4.11 lists the average computing times needed for 2000 method evaluations, contrasting the performances of the CC + Map and $\mathrm{CC}+$ Attr method variants. Computing attributes requires substantially more computing time (Intel ${ }^{\circledR}$ Xeon ${ }^{\circledR}$ E5-2643 $3.5 \mathrm{GHz}$ processor with single-core processing). Attribute calculations were done incrementally in the $\mathrm{CC}$ framework, which is more efficient than calculating attributes independently for each new level of the local range parameter. The optimal achieved parameter values are also reported. Similar to related work [6], near optimal parameter value combinations exist owing to segmentation algorithm and mapping function characteristics.

Following on from Table 4.11, Figure 4.11 shows the averaged fitness profiles for the various problems and corresponding metrics, prior to cross-validation. Specifically, note the slightly slower start of mapping function method variants compared with attribute variants; however, they ultimately lead to better results (and in the first two problems start off better). In terms of search method progression, some variation exists based on the difficulty of the problem. Generally all variants converged more slowly in the Hagadera problem ("difficult") compared with the Bokolmanyo problem. Note the variations in optimal results compared with cross-validated values (Tables 4.8-10), specifically considering the RBSB metric with its compact formulation. The figure also highlights the fact that the more complex method variants obtain superior results relatively quickly in the search processes - useful information if method execution times need to be short. 
Finally, and most significantly, the results reported in Tables 4.8-10 are augmented with a Friedman rank test [61] to give a generalized and discrete indication of the usefulness of the method variants.

The Friedman rank test is a simple non-parametric test ranking multiple methods (e.g., CC, CC + Map, etc.) over multiple problems/data sets. The rank test was run on the four method variants considering the various problems and metric conditions (36 in total, cross-validated). A Nemenyi post hoc test was also conducted to test whether critical differences exist. Figure 4.12 illustrates this result, with the confidence interval set to $95 \%$ and a critical difference of 0.349 (ranking) generated. Note that the figure shows results under cross-validated conditions.

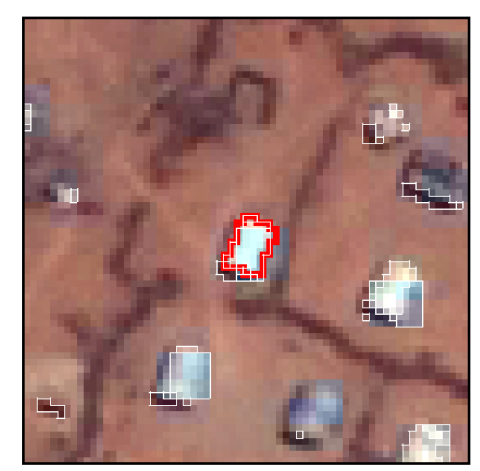

(a)

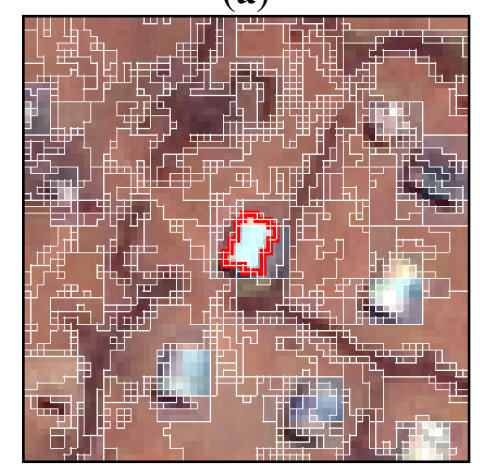

(d)

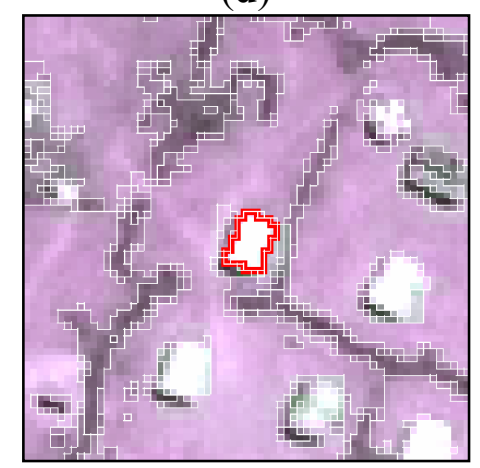

(g)

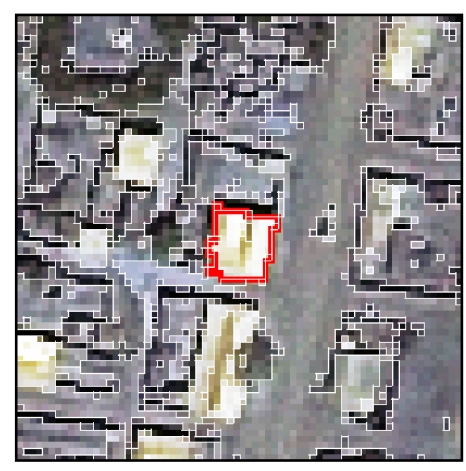

(b)

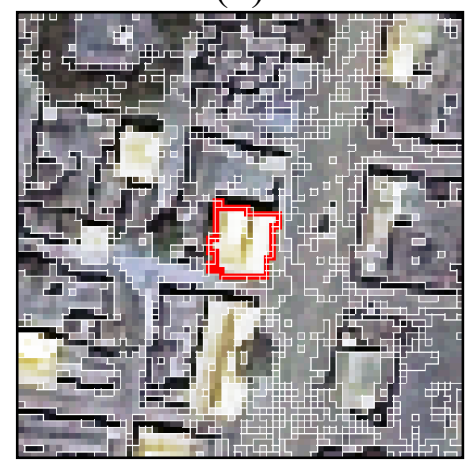

(e)

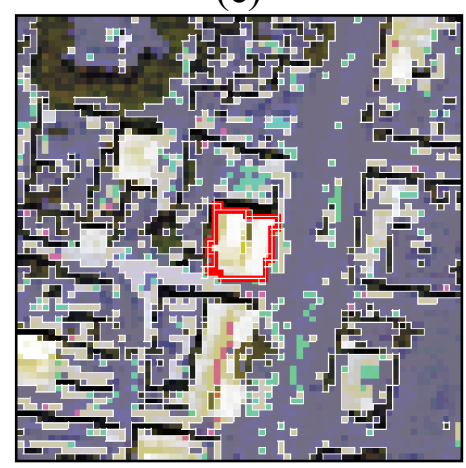

(h)

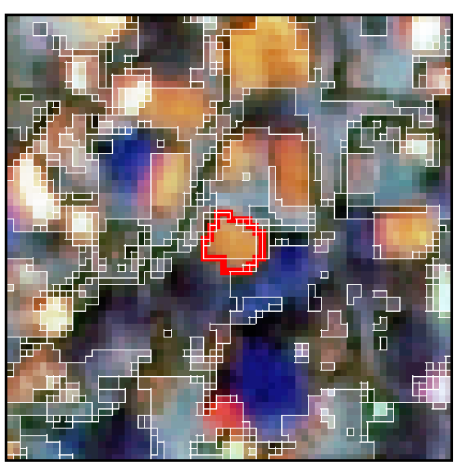

(c)

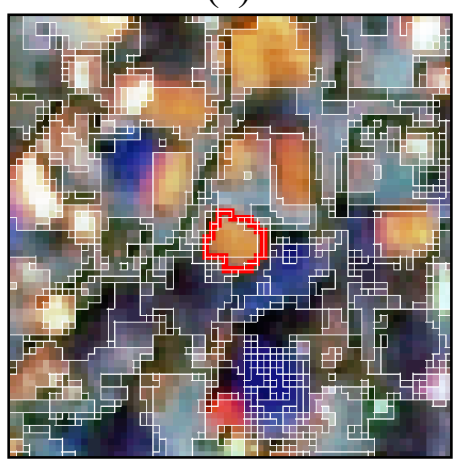

(f)

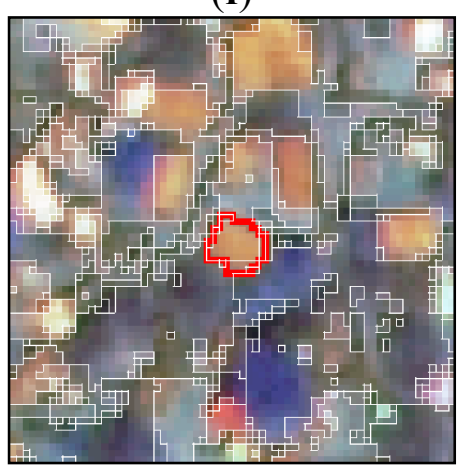

(i)

Figure 4.10. Cont. 


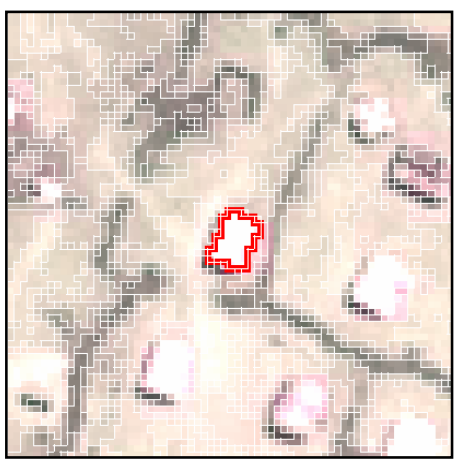

(j)

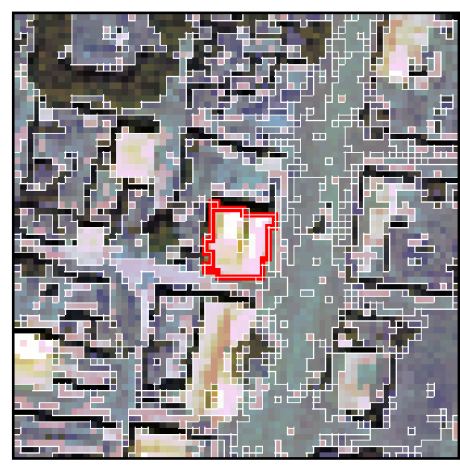

(k)

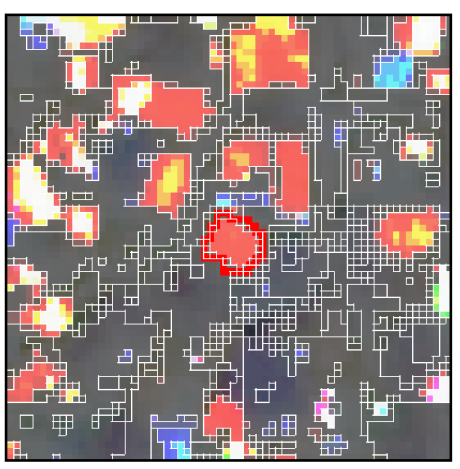

(1)

Figure 4.10. Exemplar optimal segmentation results focused on a random reference segment. The rows depict the CC, CC + Attr, CC + Map, and CC + Attr + Map method variants respectively (in order). The columns denote the three problems, Bokolmanyo, Jowhaar, and Hagadera (in order). (a) RWJ: 0.574; (b) RWJ: 0.524; (c) RWJ: 0.787; (d) RWJ:0.683; (e) RWJ: 0.524; (f) RWJ: 0.806; (g) RWJ: 0.104; (h) RWJ: 0.506; (i) RWJ: 0.787; (j) RWJ: 0.063; (k) RWJ: 0.437; (l) RWJ: 0.549.

Table 4.11. Average computing times for experimental runs and resulting method parameters. Note the increased computing time of method variants employing attributes.

\begin{tabular}{|c|c|c|c|c|c|c|c|c|c|}
\hline Problem & $\begin{array}{l}\text { Method } \\
\text { Variant }\end{array}$ & Time & Alpha & WGlobal & Area & Std & Perimeter & Smoothness & Compactness \\
\hline \multirow[t]{2}{*}{ Bokolmanyo } & $\begin{array}{l}\mathrm{CC}+ \\
\text { Map }\end{array}$ & $\begin{array}{l}2062.304 \\
\pm 248.996\end{array}$ & $\begin{array}{l}187.600 \\
\pm 68.646\end{array}$ & $\begin{array}{c}53.600 \pm \\
15.601\end{array}$ & NA & NA & NA & NA & NA \\
\hline & $\begin{array}{l}\mathrm{CC}+ \\
\text { Attr }\end{array}$ & $\begin{array}{r}3083.551 \\
\pm 237.328 \\
\end{array}$ & $\begin{array}{r}173.300 \\
\pm 66.331 \\
\end{array}$ & $\begin{array}{c}196.000 \pm \\
44.838 \\
\end{array}$ & $\begin{array}{c}247.500 \pm \\
142.417 \\
\end{array}$ & $\begin{array}{c}50.442 \pm \\
58.470 \\
\end{array}$ & $\begin{array}{c}369.900 \pm \\
196.794 \\
\end{array}$ & $\begin{array}{c}21.483 \pm \\
7.688 \\
\end{array}$ & $\begin{array}{c}19.803 \pm \\
7.439\end{array}$ \\
\hline \multirow[t]{2}{*}{ Jowhaar } & $\begin{array}{l}\mathrm{CC}+ \\
\text { Map }\end{array}$ & $\begin{array}{l}2182.659 \\
\pm 193.999\end{array}$ & $\begin{array}{r}165.900 \\
\pm 82.538\end{array}$ & $\begin{array}{c}155.200 \pm \\
19.136\end{array}$ & NA & NA & NA & NA & NA \\
\hline & $\begin{array}{l}\mathrm{CC}+ \\
\text { Attr }\end{array}$ & $\begin{array}{r}4136.116 \\
\pm 498.270 \\
\end{array}$ & $\begin{array}{c}98.900 \pm \\
52.297 \\
\end{array}$ & $\begin{array}{c}203.500 \pm \\
43.775 \\
\end{array}$ & $\begin{array}{c}392.000 \pm \\
85.249\end{array}$ & $\begin{array}{r}133.289 \\
\pm 60.647 \\
\end{array}$ & $\begin{array}{c}620.500 \pm \\
241.420 \\
\end{array}$ & $\begin{array}{c}18.379 \pm \\
6.910 \\
\end{array}$ & $\begin{array}{c}22.466 \pm \\
4.331 \\
\end{array}$ \\
\hline \multirow[t]{2}{*}{ Hagadera } & $\begin{array}{l}\mathrm{CC}+ \\
\text { Map }\end{array}$ & $\begin{array}{l}2168.177 \\
\pm 226.159\end{array}$ & $\begin{array}{r}101.700 \\
\pm 65.052\end{array}$ & $\begin{array}{c}148.300 \pm \\
29.803\end{array}$ & NA & NA & NA & NA & NA \\
\hline & $\begin{array}{l}\mathrm{CC}+ \\
\mathrm{Attr}\end{array}$ & $\begin{array}{r}5409.293 \\
\pm 352.444\end{array}$ & $\begin{array}{r}187.400 \\
\pm 68.704\end{array}$ & $\begin{array}{c}240.300 \pm \\
21.525\end{array}$ & $\begin{array}{c}342.100 \pm \\
81.266\end{array}$ & $\begin{array}{r}162.601 \\
\pm 86.782\end{array}$ & $\begin{array}{c}574.700 \pm \\
271.998\end{array}$ & $\begin{array}{c}23.573 \pm \\
7.351\end{array}$ & $\begin{array}{c}19.940 \pm \\
6.113\end{array}$ \\
\hline
\end{tabular}



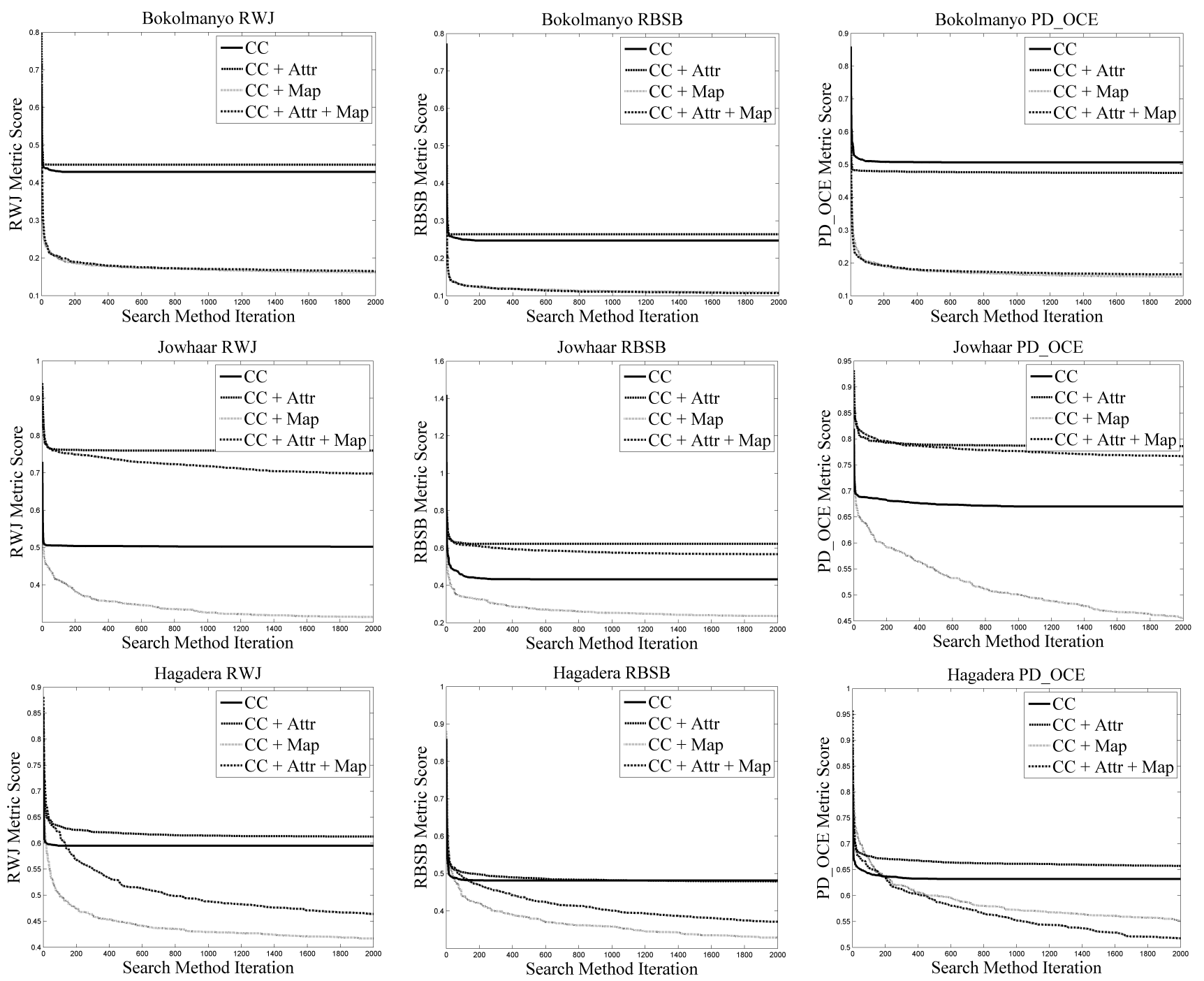

Figure 4.11. Search method profiles for the different problems under different metric conditions. Note that for the simpler Bokolmanyo problem near-optimal results are achieved relatively early on in the search process. In the more complex problems, the methods need substantially more iterations in finding the achievable optimal parameter set.

On examining Figure 4.12, the CC + Map variant of the method (using various mapping functions) ranked first, followed by the most complex method variant $(\mathrm{CC}+\mathrm{Attr}+\mathrm{Map})$. Under cross-validated conditions, adding attributes proves detrimental. The investigated problems are not exhaustive. The variants are all statistically significantly different from one another. This figure reports a general observation under extensive evaluations (50 million segment evaluations). Under a more succinct selection of attributes and problems, attributes may well be more useful. The figure suggests simple data mapping functions should be a worthwhile consideration in method design within this general framework. Mapping functions may be considered (indirect means of changing connectivity type), but other more direct means of defining connectivity (parameterizable) may also prove useful. This is in addition to such a variant requiring less computing time, compared with computing additional attributes. 


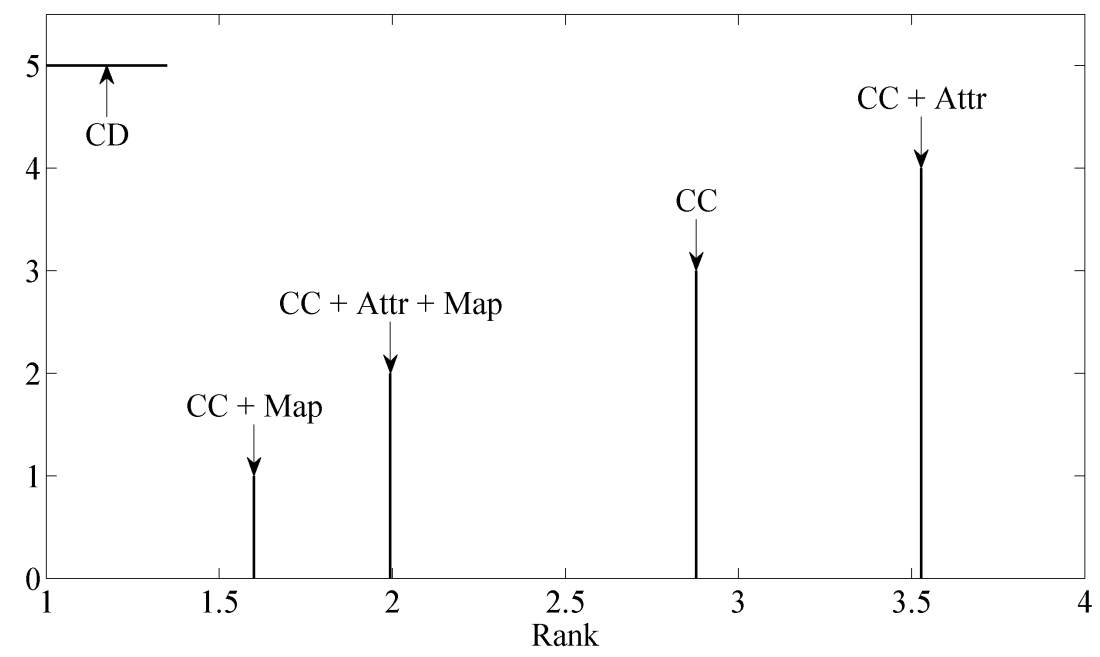

Figure 4.12. Friedman rank test with a Nemenyi post hoc test conducted on results from Tables 4.8-10. Confidence interval is set to 95\%. A Critical Difference (CD) of 0.349 is generated (ranking). All method variants deliver statistically significant different results. Generally speaking, the CC + Map method variant was found most useful.

\subsection{Conclusions}

In this work a general method in the context of sample supervised segment generation was examined. Such general methods aim to generate thematically accurate image segments, easing further processing and increasing final classification accuracies. The method incorporates a graph-based segmentation method, with constraining attributes and data mapping functions providing additional flexibility in the nature of the generated segments. These additional constituents to the method were profiled for their relative utility in enhancing the quality of the generated segments. It was found that constraining attributes, conjectured to be useful, did not add value to segment quality. Data mapping functions proved more useful in this regard, generating better quality segments consistently. Other constituents could also be added to such a method, but other formulations of defining connectivity in such an approach could be most beneficial. Various other approaches to changing connectivity could be considered as opposed to mapping functions, including analyzing scene-wide connectivity properties, considering hypo/hyper connectivity definitions, and defining connectedness as part of the optimization problem.

A few experimental considerations should be noted with such a method. Various processes in such a general approach may be stochastic, not only the given metaheuristic. In this particular instance the segmentation algorithm generates unique or repeatable results. This might not always be the case. Adding numerous attributes, without a priori known usefulness, should be avoided as additional search landscape dimensionality increases problem difficulty. Future work could profile a range of spectral and geometric attributes for their usefulness in various problems (land-cover element specific). Using various segment-sampling sizes could also provide additional insight into method generalizability to unseen problems.

A method variant, incorporating attribute selection as part of the optimization problem, could also be considered. This would entail a combined combinatorial and real valued optimization problem. The utilized metrics also need careful consideration, as various metrics will have different convergence 
characteristics, as shown in this work. The nature of correlations among metric scores and the ease of subsequent processes or classification results are also open to research. Note that the presented method, inherently hierarchical, functions on a singular segmentation level and attempts to find a level appropriate to the given problem or elements of interest in the scene. Hierarchical aspects were not considered.

The proposed method and results add to the discussion on supervised methods for segment generation in a remote sensing context. Applications such as rapid mapping or emergency response mapping may benefit from such approaches. Another application may be targeted land-cover element identification, incorporating single-class classification algorithms. User-driven image analysis approaches, found within the context of Geographic Object Based-Image Analysis (GEOBIA), might benefit from such sample supervised segment generation methods. How methods such as the one presented here, based on modular image segmentation, may efficiently synergize with more complete workflows [62], including classification processes [54,63] or larger automated image analysis methods, should be a worthwhile investigation.

\section{Acknowledgments}

This work has been conducted under the GIONET project funded by the European Commission, Marie Curie Program, Initial Training Networks, Grant Agreement No. PIT-GA-2010-264509. The programming libraries OpenCV, Qt, SwarmOps, and Terralib, and the MATLAB sparse representation toolbox were used in this work, with the method programmed in $\mathrm{C}++$.

\section{Conflicts of Interest}

The author declares no conflict of interest.

\section{References}

1. Blaschke, T.; Hay, G.J.; Kelly, M.; Lang, S.; Hofmann, P.; Addink, E.; Feitosa, R.Q.; van der Meer, F.; van der Werff, H.; van Coillie, F.; et al. Geographic object-based image analysis-Towards a new paradigm. ISPRS J. Photogramm. Remote Sens. 2014, 87, 180-191.

2. Soille, P.; Pesaresi, M. Advances in mathematical morphology applied to geoscience and remote sensing. IEEE Trans. Geosci. Remote Sens. 2002, 40, 2042-2055.

3. Fourie, C.; Schoepfer, E. Connectivity thresholds and data transformations for sample supervised segment generation. In Proceedings of the IEEE International Geoscience and Remote Sensing Symposium (IGARSS 2013), Melbourne, Australia, 21-26 July 2013.

4. Bhanu, B.; Lee, S.; Ming, J. Adaptive image segmentation using a genetic algorithm. IEEE Trans. Syst. Man Cybern. 1995, 25, 1543-1567.

5. Feitosa, R.Q.; Costa, G.A.O.P.; Cazes, T.B.; Feijo, B. A genetic approach for the automatic adaptation of segmentation parameters. In Proceedings of the Geographic Object-Based Image Analysis (GEOBIA 2006), Salzburg, Austria, 4-5 July 2006.

6. Fourie, C.; Schoepfer, E. Data transformation functions for expanded search spaces in geographic sample supervised segment generation. Remote Sens. 2014, 5, 3791-3821. 
7. Derivaux, S.; Forestier, G.; Wemmert, C.; Lefèvre, S. Supervised image segmentation using watershed transform, fuzzy classification and evolutionary computation. Pattern Recognit. Lett. 2010, 31, 2364-2374.

8. Soille, P. Constrained connectivity for hierarchical image partitioning and simplification. IEEE Trans. Pattern Anal. Mach. Intell. 2008, 30, 1132-1145.

9. Ouzounis, G.K.; Soille, P. The Alpha-Tree Algorithm; JRC Scientific and Policy Report; Publications Office of the European Union: Luxembourg City, Luxembourg, 2012.

10. Soille, P. Constrained connectivity for the processing of very-high-resolution satellite images. Int. J. Remote Sens. 2010, 31, 5879-5893.

11. Najman, L. On the equivalence between hierarchical segmentations and ultrametric watersheds. J. Math. Imaging Vis. 2011, 40, 231-247.

12. Soille, P. Preventing chaining through transitions while favouring it within homogeneous regions. In Mathematical Morphology and Its Applications to Image and Signal Processing; Springer: Berlin, Germany, 2011; pp. 96-107.

13. Soille, P.; Grazzini, J. Advances in constrained connectivity. In Proceedings of the 14th IAPR International Conference, DGCI 2008, Lyon, France, 16-18 April 2008.

14. Gueguen, L.; Soille, P. Frequent and dependent connectivities. In Mathematical Morphology and its Applications to Image and Signal Processing; Springer: Berlin, Germany, 2011; pp. 120-131.

15. Gueguen, L.; Velasco-Forero, S.; Soille, P. Local mutual information for dissimilarity-based image segmentation. J. Math. Imaging Vis. 2014, 48, 625-644.

16. Perret, B.; Lefèvre, S.; Collet, C.; Slezak, É. Hyperconnections and hierarchical representations for grayscale and multiband image processing. IEEE Trans. Image Process. 2012, 21, 14-27.

17. Ouzounis, G.K.; Wilkinson, M.H. Hyperconnected attribute filters based on k-flat zones. IEEE Trans. Pattern Anal. Mach. Intell. 2011, 33, 224-239.

18. Wilkinson, M.H. An axiomatic approach to hyperconnectivity. In Mathematical Morphology and its Application to Signal and Image Processing; Springer: Berlin, Germany, 2009; pp. 35-46.

19. Soille, P. Morphological Image Analysis: Principles and Applications; Springer-Verlag: New York, NY, USA, 2003.

20. Najman, L.; Cousty, J. A graph-based mathematical morphology reader. Pattern Recognit. Lett. 2014, 47, 3-17.

21. Tuia, D.; Pacifici, F.; Kanevski, M.; Emery, W.J. Classification of very high spatial resolution imagery using mathematical morphology and support vector machines. IEEE Trans. Geosci. Remote Sens. 2009, 47, 3866-3879.

22. Kemper, T.; Jenerowicz, M.; Pesaresi, M.; Soille, P. Enumeration of dwellings in darfur camps from GeoEye-1 satellite images using mathematical morphology. IEEE J. Sel. Top. Appl. Earth Observ. Remote Sens. 2011, 4, 8-15.

23. Salembier, P.; Serra, J. Flat zones filtering, connected operators, and filters by reconstruction. IEEE Trans. Image Process. 1995, 4, 1153-1160.

24. Breen, E.J.; Jones, R.; Talbot, H. Mathematical morphology: A useful set of tools for image analysis. Stat. Comput. 2000, 10, 105-120. 
25. Pedergnana, M.; Marpu, P.R.; Dalla Mura, M.; Benediktsson, J.A.; Bruzzone, L. A novel technique for optimal feature selection in attribute profiles based on genetic algorithms. IEEE Trans. Geosci. Remote Sens. 2013, 51, 3514-3528.

26. Pesaresi, M.; Benediktsson, J.A. A new approach for the morphological segmentation of high-resolution satellite imagery. IEEE Trans. Geosci. Remote Sens. 2001, 39, 309-320.

27. Dalla Mura, M.; Atli Benediktsson, J.; Waske, B.; Bruzzone, L. Extended profiles with morphological attribute filters for the analysis of hyperspectral data. Int. J. Remote Sens. 2010, 31, 5975-5991.

28. Fauvel, M.; Benediktsson, J.A.; Chanussot, J.; Sveinsson, J.R. Spectral and spatial classification of hyperspectral data using svms and morphological profiles. IEEE Trans. Geosci. Remote Sens. 2008, 46, 3804-3814.

29. Soille, P. On genuine connectivity relations based on logical predicates. In Proceedings of the 14th International Conference on Image Analysis and Processing, Modena, Italy, 10-14 September 2007.

30. Wilkinson, M.H.; Roerdink, J.B. Fast morphological attribute operations using tarjan's union-find algorithm. In Mathematical Morphology and Its Applications to Image and Signal Processing, Springer: Berlin, Germany, 2000; pp. 311-320.

31. Havel, J.; Merciol, F.; Lefèvre, S. Efficient schemes for computing $\alpha$-tree representations. In Mathematical Morphology and Its Applications to Image and Signal Processing; Hendriks, C.L., Borgefors, G., Strand, R., Eds.; Springer: Berlin, Germany, 2013; Volume 7883, pp. 111122.

32. Neubert, M.; Herold, H.; Meinel, G. Evaluation of remote sensing image segmentation quality_Further results and concepts. Int. Arch. Photogramm. Remote Sens. Spat. Inf. Sci. 2006, 36, 6-11.

33. Dalla Mura, M.; Benediktsson, J.A.; Bruzzone, L. A general approach to the spatial simplification of remote sensing images based on morphological connected filters. In Proceedings of the IEEE International Geoscience and Remote Sensing Symposium (IGARSS), Vancouver, BC, Canada, 24-29 July 2011.

34. Soille, P.; Najman, L. On morphological hierarchical representations for image processing and spatial data clustering. In Applications of Discrete Geometry and Mathematical Morphology; Springer: Berlin, Germany, 2012; pp. 43-67.

35. Evans, A.N.; Gimenez, D. Extending connected operators to colour images. In Proceedings of the 15th IEEE International Conference on Image Processing, San Diego, CA, USA, 12-15 October 2008.

36. Louverdis, G.; Vardavoulia, M.I.; Andreadis, I.; Tsalides, P. A new approach to morphological color image processing. Pattern Recognit. 2002, 35, 1733-1741.

37. Talbi, E. Metaheuristics: From Design to Implementation; Wiley: Hoboken, NJ, USA, 2009.

38. Barr, R.S.; Golden, B.L.; Kelly, J.P.; Resende, M.G.; Stewart, W.R., Jr. Designing and reporting on computational experiments with heuristic methods. J. Heuristics 1995, 1, 9-32.

39. Bartz-Beielstein, T. Experimental Research in Evolutionary Computation; Springer: Berlin, Germany, 2006. 
40. Rardin, R.L.; Uzsoy, R. Experimental evaluation of heuristic optimization algorithms: A tutorial. J. Heuristics 2001, 7, 261-304.

41. Birattari, M.; Zlochin, M.; Dorigo, M. Towards a theory of practice in metaheuristics design: A machine learning perspective. RAIRO Theor. Inform. Appl. 2006, 40, 353-369.

42. Vanneschi, L.; Mussi, L.; Cagnoni, S. Hot topics in evolutionary computation. Intell. Artif. 2011, 5, 5-17.

43. Pedersen, M. Swarmops: Black-box Optimization in Ansic; Hvass Lab.: Southampton, UK, 2008.

44. Price, K.V.; Storn, R.M.; Lampinen, J.A. Differential Evolution: A Practical Approach to Global Optimization; Springer: Berlin, Germany, 2005.

45. Kennedy, J. Particle swarm optimization. In Encyclopedia of Machine Learning, Springer: Berlin, Germany, 2010; pp. 760-766.

46. Zhang, Y.J. A survey on evaluation methods for image segmentation. Pattern Recognit. 1996, 29, 1335-1346.

47. Chabrier, S.; Emile, B.; Rosenberger, C.; Laurent, H. Unsupervised performance evaluation of image segmentation. EURASIP J. Appl. Signal Process. 2006, 2006, 1-12.

48. Freddrich, C.M.B.; Feitosa, R.Q. Automatic adaptation of segmentation parameters applied to non-homogeneous object detection. In Proceedings of the Geographic Object-based Image Analysis (GEOBIA), Calgary, AB, Canada, 29 June-2 July 2008.

49. Polak, M.; Zhang, H.; Pi, M. An evaluation metric for image segmentation of multiple objects. Image Vis. Comput. 2009, 27, 1223-1227.

50. Pignalberi, G.; Cucchiara, R.; Cinque, L.; Levialdi, S. Tuning range image segmentation by genetic algorithm. EURASIP J. Appl. Signal Process. 2003, 2003, 780-790.

51. Achanccaray, P.; Ayma, V.; Jimenez, L.; Garcia, S.; Happ, P.; Feitosa, R.; Plaza, A. A free software tool for automatic tuning of segmentation parameters. Southeast. Eur. J. Earth Observ. Geomat. 2014, 3, 707-712.

52. Cagnoni, S. Evolutionary computer vision: A taxonomic tutorial. In Proceedings of the Eighth International Conference on Hybrid Intelligent Systems (HIS 2008), Barcelona, Spain, 10-12 September 2008.

53. Feitosa, R.Q.; Ferreira, R.S.; Almeida, C.M.; Camargo, F.F.; Costa, G.A.O.P. Similarity metrics for genetic adaptation of segmentation parameters. In Proceedings of the Geographic ObjectBased Image Analysis (GEOBIA 2010), Ghent, Belgium, 29 June-2 July 2010.

54. Fourie, C.; Schoepfer, E. Classifier directed data hybridization for geographic sample supervised segment generation. Remote Sens. 2014, 6, 11852-11882.

55. Fourie, C.; Schoepfer, E. Sample supervised search-centric approaches in geographic object-based image analysis (geobia): Concepts, state-of-the-art and a future outlook. In Earth Observation for Land and Emergency Monitoring-Innovative Concepts for Environmental Monitoring from Space; Balzter, H., Ed.; Wiley-Blackwell: Hoboken, NJ, USA, 2015, in press.

56. Yoda, I.; Yamamoto, K.; Yamada, H. Automatic acquisition of hierarchical mathematical morphology procedures by genetic algorithms. Image Vis. Comput. 1999, 17, 749-760.

57. Quintana, M.I.; Poli, R.; Claridge, E. Morphological algorithm design for binary images using genetic programming. Genet. Program. Evol. Mach. 2006, 7, 81-102. 
58. Gorai, A.; Ghosh, A. Gray-level image enhancement by particle swarm optimization. In Proceedings of the NaBIC 2009 World Congress on Nature \& Biologically Inspired Computing, Coimbatore, India, 9-11 December 2009.

59. Sun, L.; Yoshida, S.; Cheng, X.; Liang, Y. A cooperative particle swarm optimizer with statistical variable interdependence learning. Inf. Sci. 2012, 186, 20-39.

60. Vesterstrom, J.; Thomsen, R. A comparative study of differential evolution, particle swarm optimization, and evolutionary algorithms on numerical benchmark problems. In Proceedings of the CEC2004 Congress on Evolutionary Computation, Portland, OR, USA, 19-23 June 2004.

61. Li, Y. Sparse Machine Learning Models in Bioinformatics. Ph.D. Thesis, University of Windsor, Windsor, ON, Canada, 2013.

62. Baatz, M.; Hoffman, C.; Willhauck, G. Progressing from object-based to object-oriented image analysis. In Object-Based Image Analysis: Spatial Concepts for knowledge-Driven Remote Sensing Applications; Blaschke, T., Lang, S., Hay, G.J., Eds.; Springer: Berlin, Germany, 2008; pp. 29-42.

63. Mylonas, S.; Stavrakoudis, D.; Theocharis, J.; Mastorocostas, P. A region-based genesis segmentation algorithm for the classification of remotely sensed images. Remote Sens. 2015, 7 , 2474-2508.

(C) 2015 by the author; licensee MDPI, Basel, Switzerland. This article is an open access article distributed under the terms and conditions of the Creative Commons Attribution license (http://creativecommons.org/licenses/by/4.0/). 


\title{
CHAPTER 5: CLASSIFIER DIRECTED DATA HYBRIDIZATION FOR GEOGRAPHIC SAMPLE SUPERVISED SEGMENT GENERATION
}

\author{
Fourie, C \& Schoepfer, E \\ German Remote Sensing Data Center (DFD), German Aerospace Center (DLR), \\ 82234 Oberpfaffenhofen.
}

Published in:

Remote Sensing (2014), 6, 11852-11882

Received: 20 August 2014; in revised form: 11 November 2014 / Accepted: 18 November 2014 / Published: 28 November 2014 doi:10.3390/rs61211852 
Remote Sens. 2014, 6, 11852-11882; doi:10.3390/rs61211852

Article

\title{
Classifier Directed Data Hybridization for Geographic Sample Supervised Segment Generation
}

\section{Christoff Fourie * and Elisabeth Schoepfer}

German Remote Sensing Data Center (DFD), German Aerospace Center (DLR), 82234 Oberpfaffenhofen, Germany; E-Mail: elisabeth.schoepfer@dlr.de

* Author to whom correspondence should be addressed; E-Mail: fourie.christoff@gmail.com; Tel.: +27-72-082-4528; Fax: +49-8153-28-1445.

External Editors: Lizhe Wang and Prasad S. Thenkabail

Received: 20 August 2014; in revised form: 11 November 2014 / Accepted: 18 November 2014 / Published: 28 November 2014

\begin{abstract}
Quality segment generation is a well-known challenge and research objective within Geographic Object-based Image Analysis (GEOBIA). Although methodological avenues within GEOBIA are diverse, segmentation commonly plays a central role in most approaches, influencing and being influenced by surrounding processes. A general approach using supervised quality measures, specifically user provided reference segments, suggest casting the parameters of a given segmentation algorithm as a multidimensional search problem. In such a sample supervised segment generation approach, spatial metrics observing the user provided reference segments may drive the search process. The search is commonly performed by metaheuristics. A novel sample supervised segment generation approach is presented in this work, where the spectral content of provided reference segments is queried. A one-class classification process using spectral information from inside the provided reference segments is used to generate a probability image, which in turn is employed to direct a hybridization of the original input imagery. Segmentation is performed on such a hybrid image. These processes are adjustable, interdependent and form a part of the search problem. Results are presented detailing the performances of four method variants compared to the generic sample supervised segment generation approach, under various conditions in terms of resultant segment quality, required computing time and search process characteristics. Multiple metrics, metaheuristics and segmentation algorithms are tested with this approach. Using
\end{abstract}


the spectral data contained within user provided reference segments to tailor the output generally improves the results in the investigated problem contexts, but at the expense of additional required computing time.

Keywords: geographic object-based image analysis; segmentation; classification; sample supervised; spatial metrics; metaheuristics

\subsection{Introduction}

Remotely sensed satellite imagery has unique characteristics and derived information products compared to imagery encountered in many other image analysis disciplines. Various sub-elements in such imagery may need to be recognized and their attributes quantified. Land-cover mapping is a common task in this context, where it is attempted to generate a partial or full description of a given area from Earth observation imagery, with an emphasis on element geometric and thematic accuracies. Geographic Object-Based Image Analysis (GEOBIA) has emerged as a viable avenue of approaches, or paradigm, to tackle such remote sensing image analysis tasks [1-4] due to the common spectral-textural-geometric and thematic correlations of elements of interest in satellite imagery [5-7].

Incorporating a segmentation algorithm, which is central in many GEOBIA approaches, either for semantic object segmentation and description [8], or for only allowing for the generation of richer attributes for classification, have been shown to be efficient in many real world applications [2]. This is partly due to the h-res phenomenon [9] encountered commonly when concerned with Very High Resolution (VHR) optical imagery, where the spatial resolution of captured imagery is finer than the geometry of the elements of interest and pixel-based discriminative methods are limited to produce adequate results (due to the so called salt-and-pepper effect [10]). Also, such fidelity in resolution may be needed to identify elements, but intra element spectral variability may additionally cause problems in this process [11]. Segmentation algorithms allowing for spatial aggregation in addition to observing spectral characteristics have been shown to be efficient in working towards identifying elements [7,11-14]. Based on the characteristics of the desired information products and the nature of the data, the availability of commercial and freeware GEOBIA software [2,15-17] and the extent of the literature $[1,2,4]$, it is shown that GEOBIA is a promising paradigm [1].

Although thematically accurate segments are commonly aimed for in a GEOBIA workflow, adequate segmentation is problematic to attain for single or multiclass elements using only a single pass of a given segmentation algorithm. This may be due to the complexity of the scene, especially when thematic and spectral correlations start to diverge, and limitations of the given segmentation algorithm. Various general approaches have been proposed to address the challenge of thematically accurate image segmentation and classification (semantic segmentation), including advocating rule-set or expert system's approaches within GEOBIA [1,14], the development of new domain specific segmentation algorithms [18], multi-scale image analysis [6,19,20], using context information or spatial relationships among segments [14,21,22], and hybridizing or interleaving classification and segmentation processes [23-25]. Another general approach addressing the problem of segmentation within GEOBIA casts the creation of thematically accurate image segments as a search or optimization 
problem [26-29]. In such an approach the parameters of a given segmentation algorithm is automatically tuned based on the provision of a limited amount of user provided reference segments. The geometric aspects of provided reference segments are matched with generated segments in the iterative search process via spatial metrics.

In this work a novel variant of such a sample supervised segment generation approach is presented and quantitatively evaluated. The initial concept was presented in abstract from in [30]. An enlarged search space is defined to include pixel-based classification processes. Derived probability images are used to direct a change in the original input imagery, such that the given segmentation algorithm may perform better on the given problem. The proposed method is compared with the generic formulation of sample supervised segment generation and results are demonstrated via the task of accurately segmenting structures in towns, villages and refugee camps on VHR optical remote sensing data. This contribution thus falls within the context of enlarged search spaces first presented in [29], but proposes a methodology that uses spectral content contained within reference segments as opposed to adding data transformation or mapping functions.

Section 5.2 gives an overview of sample supervised segment generation and reviews related work. In Section 5.3 a new variant of sample supervised segment generation is presented, incorporating classification in the segment generation process. In Section 5.4 the data used is briefly described, with the comparative experimental methodologies outlined in Section 5.5. In Section 5.6 results are presented and discussed. Prospects and limitations are highlighted in Section 5.7.

\subsection{Background and Related Work}

Sample supervised segment generation, or more generally sample supervised image processing/ analysis, denotes the process of automatically tuning the parameters of a given segmentation algorithm or constructing image processing operators for segment generation based on the provision of exemplar output segments. A user typically needs to digitize or provide examples of desired segmentation results. Such an approach has attracted research attention in the imaging disciplines in general [26,28,31-35] and also more specifically in the context of remote sensing image analysis [27,29,36]. It is a feasible strategy if a scene contains numerous "similar" elements that are of interest, common in many mapping tasks. Unsupervised strategies, not requiring reference segments but using scene wide image statistics, are also pursued [19]. It should be noted that the uses of efficient search methods are diverse in the imaging disciplines, with attribute selection and feature creation other common applications [37,38].

Figure 5.1 [39] illustrates the generic formulation of such an approach. A user provides a selection of reference segments or objects, typically digitized or extracted with other tools [40]. An iterative search process is invoked, where the parameter space of a given segmentation algorithm is searched. At iterations of the search process a specific parameter set is passed onto the segmentation algorithm from the optimizer. The tuned segmentation algorithm is executed on the image, typically subsets of the image covering areas around the provided reference segments. Empirical discrepancy, or spatial metrics [41] are employed to match the generated segments to that of the user provided reference segments. This process is commonly referred to as the fitness evaluation. The optimizer uses the quality score generated by the metrics to direct the next iteration of the search. The method terminates when a certain number of search iterations have passed or a certain quality threshold has been reached, although various other stopping criteria may be considered. The parameter set resulting 
in the best metric score is given as the output. Subsequently, the entire scene may be segmented with the segmentation algorithm tuned with the output parameter set.

Figure 5.1. Architecture of the generic formulation of sample supervised segment generation [39].

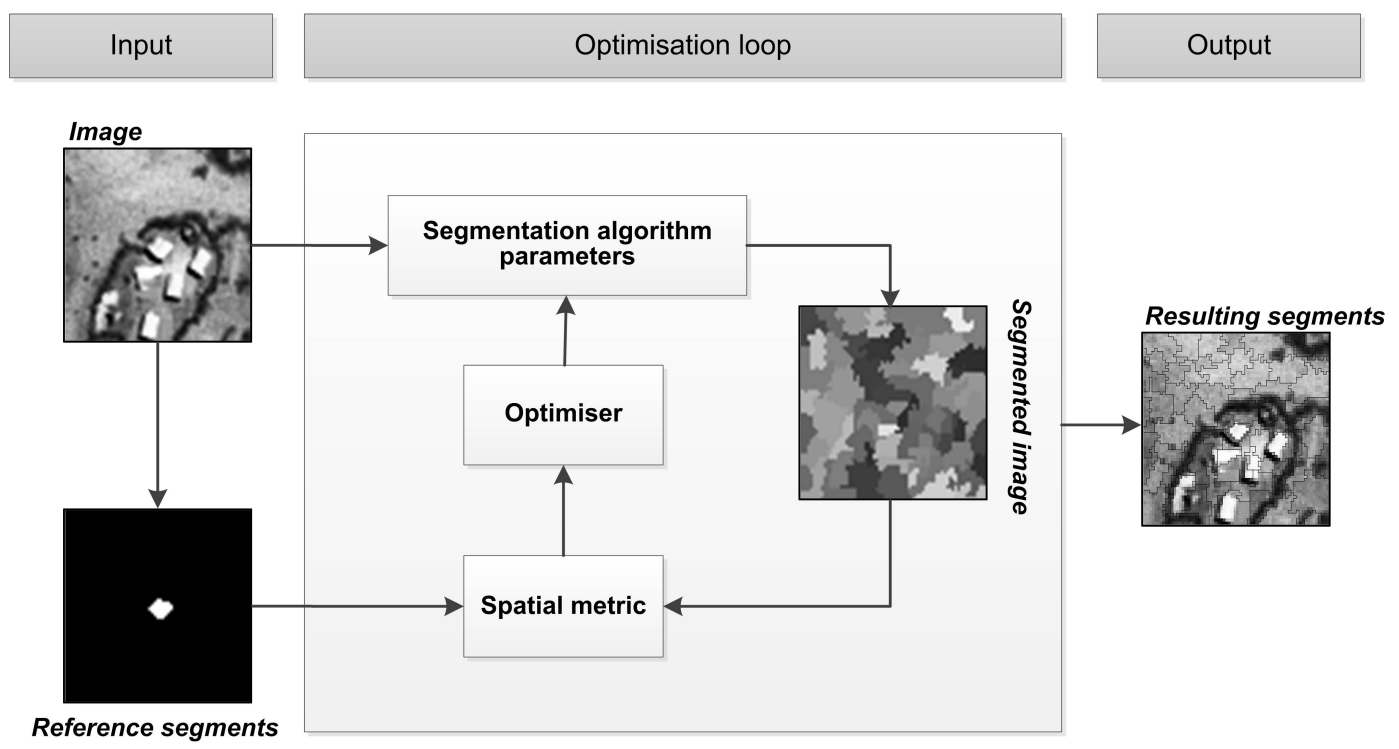

A sample supervised segment generation method typically advocates an interactive, user driven image analysis process. Segmentation is, generally computationally expensive, resulting in computationally expensive fitness evaluations. Searching the parameter space efficiently was a major driver for the development of this method [26]. Metaheuristics, which are stochastic population based search methods, are well suited and studied in the context of this general approach $[26,29,42,43]$, commonly leading to higher quality fitness scores in less search time compared to more general search strategies. Such a general approach may also be used to compare segmentation algorithms for a given task, or purely to test the general feasibility of a given algorithm for a given task. Also, this approach may find use alongside other, more encompassing, image analysis strategies. It could be used to work towards a final product in complex scene scenarios or used alongside traditional GEOBIA approaches such as rule set development [14].

Research on this general method typically aims for generating better quality results in less time. Specific aspects investigated include the evaluation of the performances of different search methods [29,42-44], the applicability of various empirical discrepancy metrics (fitness functions) [29,36], the performances of various segmentation algorithms in such an approach $[29,42,45-47]$ and the extension of the concept to more modular image processing methods [24,45-50]. Uncertainties remain surrounding the generalizability of such a method, its sampling size requirements and whether strong correlations exist between classification results and segmentation [27,29]. Research and freeware software in this vein are available [29,42,51]. Having some a priori knowledge on the capability of the segmentation algorithm seems necessary [27].

The search landscape may also be extended to include processes surrounding the core segmentation that may lead to better quality segments or classification results $[29,45,48]$. A search landscape defines the n-dimensional surface of discrepancy metric results for all parameter value combinations, where $n$ is the number of parameters in the method. Additional processes may tailor the data to allow a given 
segmentation algorithm to perform better, for example by adding extra data transformation functions [29], or by automatically performing post segmentation processes to further improve results. Such processes may be interdependent [52] with segment generation and should subsequently be optimized simultaneously or interdependently with the segmentation algorithm parameters.

\subsection{Classifier Directed Data Hybridization}

\subsubsection{Exploiting Spectral Data Contained within Provided Reference Segments for Segment} Generation

Reference segment geometric properties are most commonly queried to drive the search process in sample supervised segment generation approaches [26-28]. Such reference segments also contain or encapsulate spectral data, which is implicitly provided. Figure 5.2 illustrates the two basic properties or aspects derived from provided reference segments. The question is raised and explored how the spectral data contained within the provided reference segments (Figure 5.2. Arrow B) may contribute to generating more accurate image segments (Figure 5.2. Arrow A) via data modification processes (Figure 5.2. Arrow C).

Figure 5.2. The rationale behind the proposed method. Segment geometry is commonly provided via digitizing and used to drive a parameter search process (Arrow A) for segment generation. Reference segment spectral content (Arrow B), which is implicitly provided, is queried to influence a data transformation (Arrow C) affecting segment generation.

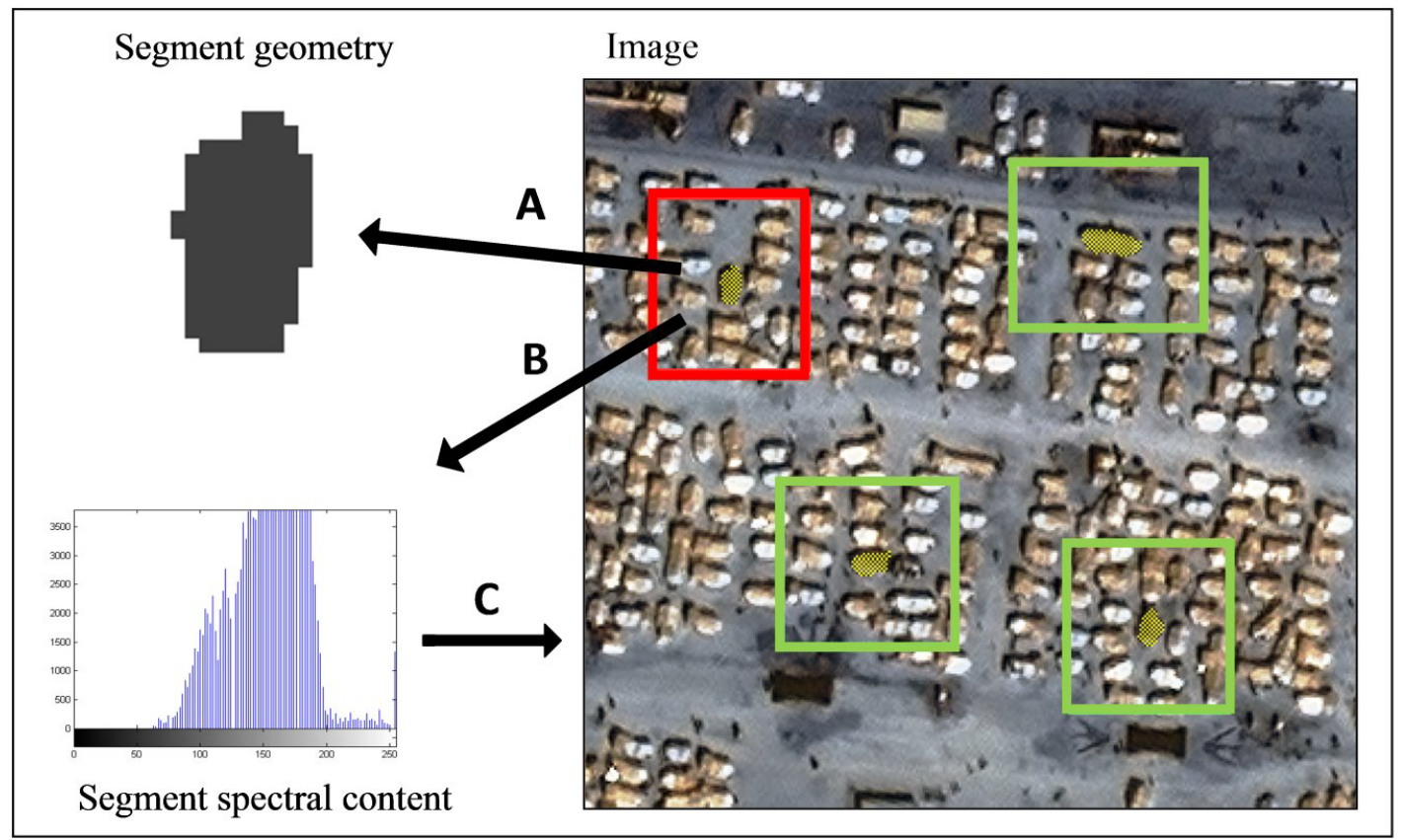

It is suggested that pixel based classification methods, although having their limitations compared to object-based image analysis approaches [1], provide useful information in clustering thematic elements in imagery in many instances. Classification in this context is used to assist in segmentation and not for thematic element identification. It is proposed to interleave classification and segmentation processes via an expanded search landscape in the context of sample supervised segment generation. 
This proposition is inspired by methods that interleave classification and segmentation for thematic element identification purposes [23,53-55] and methods defining expanded search landscapes in sample supervised segment generation $[29,45]$. The spatial/spectral aggregation of segmentation is complimented with the discriminative power of a classification process. Classification is used to tailor the data, so that the given segmentation algorithm performs better. One-class classifiers or novelty detectors having strong discriminative power such as a one-class Support Vector Machine (SVM) [56]/support vector domain descriptor [57] and others [58], may be employed to generate a preliminary classification or probability image (with additional processes, described below) of pixel membership based on the spectral content encapsulated within provided reference segments. Such an initial classification may provide useful information in describing thematic and spectral similarities that may assist in segmentation. On the other hand, it is possible that such information may be detrimental to subsequent segmentation processes, a scenario that may be found in the context of VHR optical data. An example could be accurately segmenting all cars in a parking lot, with the large variation in spectral content causing problems for such a method. Many method variants are possible based on this basic idea. A specific formulation is presented below.

\subsubsection{Method Overview}

Figure 5.3 (source: illustrated abstract, [30]) illustrates the architecture of the proposed method variant. As with the generic formulation of such an approach (Figure 5.1), a user provides a set of reference segments as input (multiple reference segments). In this variant, the spectral data of the provided reference segments are also collected. This variant requires the same amount of user interaction, and from a user's perspective requires no additional operations compared to the generic formulation.

Figure 5.3. Architecture of the variant of sample supervised segment generation incorporating classifier directed data hybridization [30].

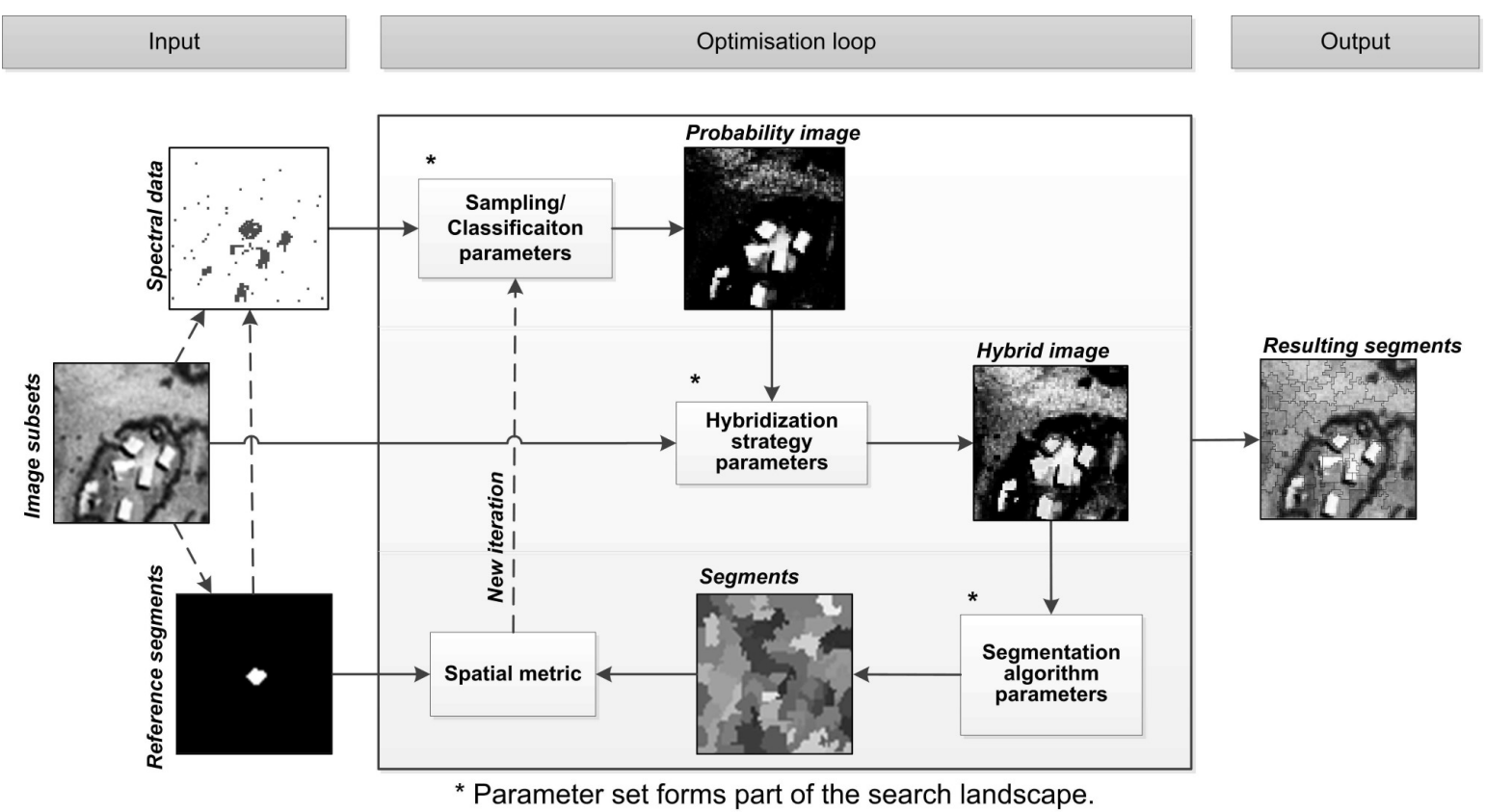


The optimization loop contains three sub-components controlled by three real-valued parameter sets. Firstly the provided spectral data are used in a synthetic sample generation and classification process to generate a probability image, detailed in Section 5.3.3. In this implementation this sub-component is controlled with four real valued parameters. Secondly, the generated probability image is fused or hybridized with the original input image. Four strategies are investigated, detailed in Section 5.3.4, including only using the probability image for segmentation purposes. This sub-component is controlled by a variable number of parameters, depending on the implementation details (between zero and four). In the third sub-component in the search process, the hybridized image is segmented with a given segmentation algorithm (algorithms detailed in Section 5.3.5). The segmentation results are evaluated with spatial metrics against the user provided reference segments (detailed in Section 5.3.6), with the evaluation result passed on to the optimizer (optimizers detailed in Section 5.3.6), which subsequently initiates a new iteration of the search process. In this implementation the method terminates when 2000 search iterations have passed, which was found a sufficient number of runs in preliminary experimentation and in related work $[29,43]$. Other termination conditions may be considered.

Figure 5.4 illustrates an example encoding of a search landscape defined in the method. The optimizer considers probability sampling and classification parameters for probability image generation, image hybridization process control parameters and segmentation algorithm parameters. This results in search landscape dimensions ranging from six to eleven, depending on implementation details. Integer/discrete parameters are converted to real. Example parameter encodings are also illustrated for each sub-component (detailed in following sections). Parameter domain interdependencies are demonstrated in the results section, necessitating the creation of such enlarged search spaces.

Figure 5.4. An example parameter set, forming the search landscape with interdependent real-valued parameter domains [30].

\begin{tabular}{|c|c|c|c|}
\hline \multicolumn{4}{|c|}{ Search landscape } \\
\hline Parameter domain: & $\begin{array}{l}\text { Sampling/Classification } \\
\text { (Section 3.3) }\end{array}$ & $\begin{array}{l}\text { Hybridization strategy } \\
\text { (Section 3.4) }\end{array}$ & $\begin{array}{l}\text { Segmentation } \\
\text { (Section 3.5) }\end{array}$ \\
\hline $\begin{array}{l}\text { Example parameter encoding: } \\
\text { (parameter explanations given in } \\
\text { sections } 3.3 .-3.5 \text {.) }\end{array}$ & $\begin{array}{l}\text { Probability image generation: } \\
\text { 1. One-class SVM nu } \\
\text { 2. One-class SVM gamma } \\
\text { 3. Two-class SVM C } \\
\text { 4. Two-class SVM gamma }\end{array}$ & $\begin{array}{l}\text { Central positions strategy: } \\
\text { 5. Probability weight } \\
\text { 6. Central position } 1 \\
\text { 7. Central position } 2 \\
\text { 8. Central position } 3\end{array}$ & $\begin{array}{l}\text { Multiresolution segmentation: } \\
\text { 9.Scale } \\
\text { 10. Colour/Shape } \\
\text { 11. Compactness/Smoothness }\end{array}$ \\
\hline
\end{tabular}

The method was implemented as a graphical user interface driven application programmed in $\mathrm{C}++$, making use of various open source libraries (in acknowledgements). Examining the progress of the automatic parameter tuning process and manually overriding proceedings are possible. Due to the envisaged usage scenario of such an approach, the method is embedded in an exemplary larger workflow containing basic scene wide segmentation and one-class classification functionality.

\subsubsection{Search Landscape: Sampling/Classification Sub-Component}

The sampling and classification sub-component entails the parameterization of the process of probability image generation. This can be implemented in various ways, with varying number of 
parameters and resultant search landscape characteristics. Parameter controlled variation in output is conjectured be most useful. This process is implemented here as follows:

A one-class SVM [56] with a Radial Basis Function (RBF) kernel is run on the spectral samples (normalized) collected from within all provided reference segments. Figure 5.5a illustrates a subset of a three-band VHR optical image where the aim would be to accurately segment all bright sink roofed structures. The sloped roofs appear spectrally diverse due to the different light reflectance angles. The red polyline in Figure 5.5a illustrates one of the digitized reference segments or objects provided by a user. This classification process is controlled by two real valued parameters (nu and gamma, see [56] for details).

Figure 5.5. Image processing conducted to derive a probability image. (a) Illustrates a subset with a delineated reference segment; (b) the masking and synthetic sampling procedure and (c) the generated probability image.

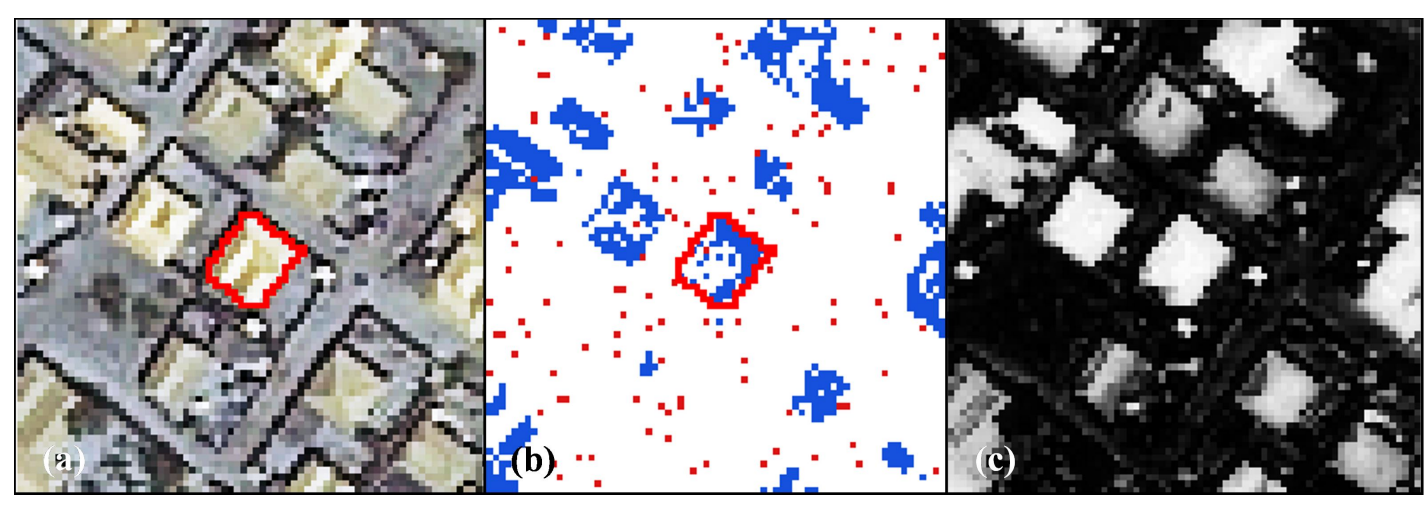

This initial classification is used as a mask to prevent the automatic selection or querying of pixels, representing a synthetic secondary class. Figure 5.5b illustrates blue shaded pixels, which constitute the classification result of the one-class classification process that acts as a mask. The red pixels represent samples taken from a linked list of random samples (generated only once). The red samples match the quantity of pixels found within the reference segments. As the nature of the mask changes (via tuning the parameters of the one-class SVM), some samples selected for the synthetic secondary class may be masked out and new samples are placed, taken from samples in the linked list. In other words, the parameters of the one-class SVM control the pixel sampling (red pixels) of a synthetic secondary class via the creation of a mask (blue pixels).

Subsequently the two sample groups with identical number of pixels are used in a two-class SVM [59] classification process to generate a probability image, illustrated in Figure 5.5c. The two controlling parameters ( $\mathrm{C}$ and gamma) are not as sensitive or do not result in significant changes in results compared to the effect of the masking/sampling process. Thus, four parameters control the nature of the probability image, with an optional additional parameter controlling the weight of the interaction in the subsequent process. These parameters encompass the probability image sub-component as illustrated in Figure 5.3. The parameter range for nu is set to [0, 0.2] and for $\mathrm{C}$ and gamma to $[0,100]$. Optionally, additional parameters may control the sampling of a synthetic secondary class to create more diversity in the generated probability output. It should be noted that the quality or accuracy of the generated probability imagery is not measured or quantified. Various other implementations are possible. 


\subsubsection{Search Landscape: Hybridization Sub-Component}

Within the image hybridization sub-component the original input image is modified, guided or directed by the classification results of the probability image. The search process can control the nature and degree of the interaction. This allows for useful aspects to be used from both the imagery. After image segments are generated, the original image should be queried for further image processing and classification processes and not the hybridized image. Three variants of image hybridization are presented and tested. In addition, the probability image itself may be considered for segmentation. Figure 5.6 illustrates arbitrary results generated by the three variants detailed below. Other variants are possible.

Figure 5.6. The three variants of data hybridization investigated. (a) Illustrates a hybrid image generated via the (a) band replacement strategy (Section 5.3.4.1); the (b) move to average strategy (Section 5.3.4.2) and (c) the move to central positions strategy (Section 5.3.4.3).

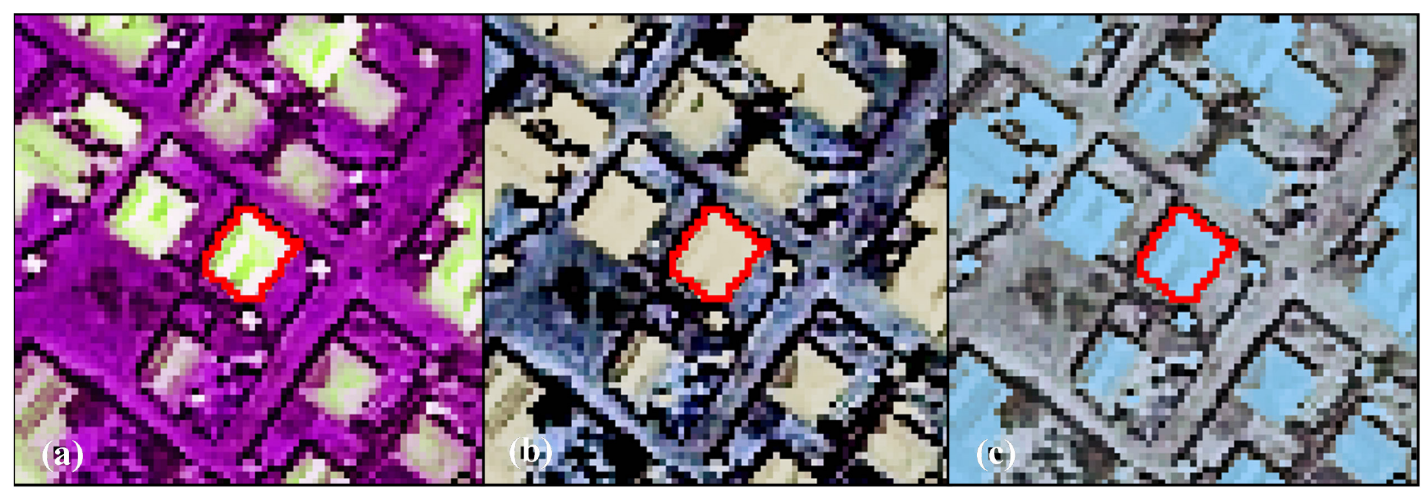

\subsubsection{Hybrid:EB (Exchange Band)}

The simple Hybrid:EB (Exchange Band) hybridization strategy replaces band $\mathrm{x}$ of the input image with the generated probability image (Figure 5.6a). For experimental conformity all used imagery in this work has three bands, with band two exchanged for the probability image. Simply adding the probability image to the image stack is also possible.

\subsubsection{Hybrid:MA (Move to Average)}

The Hybrid:MA (Move to Average) strategy (Figure 5.6b) determines the average spectral value contained in all reference segments. Pixels in the original image $(I)$ are moved towards this position based on the following equation:

$$
\text { Hybrid: } M A=I-a b s(I-A v g) \times M a g \times \operatorname{Prob}-a b s(I-A v g)
$$

where Prob denotes the probability image and $A v g$ the average spectal value, Mag is a user defined value influencing the magnitude of the move (set to two in all experiments) and $a b s$ the absolute value. An additional weighting parameter, which forms part of the optimization problem influences the intensities within Prob. Hybrid:MA is thus also a weighted function within the optimization problem. This strategy also allows for pixels to be shifted away from the calculated spectral average. 
Simply designating the average spectral value of the reference segments as the target spectral position to move pixels towards may be problematic in various problem instances.

\subsubsection{Hybrid:CP (Central Positions)}

With the Hybrid:CP (Central Positions) hybridization strategy, pixels in the original image are moved towards a parameter controlled new spectral value. The distance of this move (in percentage) is equal to the intensities in the probability image. For each band in the image a parameter is added, thus in this implementation the Hybrid:CP strategy adds an additional three parameters to the optimization problem. When concerned with 8-bit imagery, the parameter range is set to $[0,255]$. The intensity of the move is also regulated by a weighting parameter, as with the Hybrid:MA strategy. Hybrid:CP is written as:

$$
\text { Hybrid: } C P=I-(I-C P) \times P r o b
$$

where $I$ indicates the original input image, $C P$ the parameter controlled spectral position and Prob the probability image as a percentage (when concerned with 8-bit data it would be Prob/255). Figure 5.6c illustrates an arbitrary hybridized image generated with this strategy. This strategy allows for more flexibility in the hybridization, by allowing the optimizer to define the position where to pixel spectral values should be shifted (at the expense of added search landscape dimensionalities).

Figure 5.7. Segmented subsets, generated with the MS algorithm using the (a) original image, and a (b) Hybrid:CP image and segments generated with SLIC using a (c) probability image.

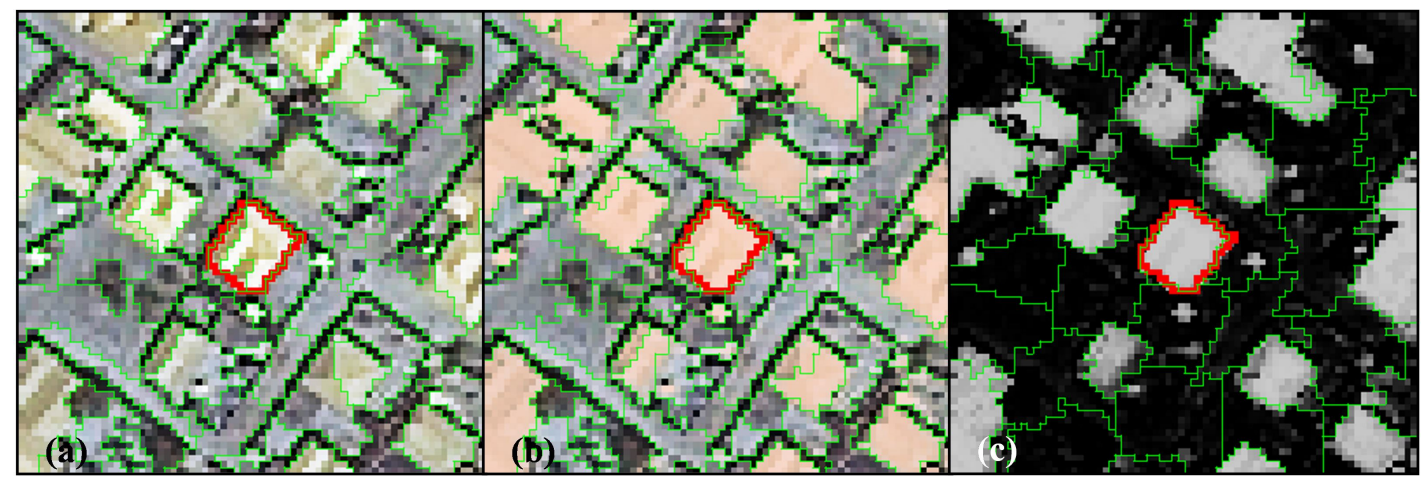

\subsubsection{Search Landscape: Segmentation Sub-Component}

The hybridized image is passed on to a given segmentation algorithm (Figure 5.3), where the optimizer also search the parameter space of said algorithm, due to parameter domain interdependencies [29,60]. In this work two segmentation algorithms are tested, namely Multiresolution Segmentation (MS) [12] and Simple Linear Iterative Clustering (SLIC) [61]. The MS algorithm adds an additional three parameters to the search landscape, with a sensitive "scale" parameter majorly responsible for the relative sizes of segments (see [12] for a full formulation). Two other parameters control the influence (Color/Shape) and definition (Compactness/Smoothness) of shape in segment generation. The MS algorithm has been extensively used in the context of GEOBIA [1,2,19,27,46]. Figure 5.7 illustrates hand tuned results of the MS algorithm run on the 
original input image (Figure 5.7a) and on the Hybrid:CP image (Figure 5.7b) and the SLIC algorithm run on the probability image (Figure $5.7 \mathrm{c}$ ).

SLIC, considered a superpixel algorithm, is commonly used in part-based models and non-thematic segmentation tasks (e.g., for purely allowing the extraction of rich attribute sets) [61]. SLIC adds two parameters to the search landscape. Similar to the MS algorithm, SLIC has a "scale" parameter controlling the relative size of generated segments [61]. Although not as efficient as the MS segmentation algorithm for creating thematically accurate segments in a remote sensing context [29], SLIC is computationally efficient allowing for more interactive segment generation in manual tuning processes.

\subsubsection{Metrics and Optimizers}

In each iteration of the method, after the image segmentation process, the generated segments are quantitatively compared with the user provided reference segments (Figure 5.3). Four spatial empirical discrepancy metrics [41] are utilized in experimentation in this work to prevent bias based on the details of any given implementation (segmentation is an ill posed problem [62] due to the variation in possible solutions). Table 5.1 summarizes the used spatial metrics, with their formulations given using set theory notation. The Reference Bounded Segments Booster (RBSB) [27] compares area offsets between the reference (R) and a generated segment (S). The Larger Segments Booster (LSB) [63] performs similarly, but considers all segments $\left(\mathrm{S}_{\mathrm{h}}\right)$ having a majority overlap with the reference segment and holds a penalization factor in the form of counting border (b) pixels intersecting the reference segment.

The Partial and Directed Object-Level Consistency Error (PD_OCE) [29,64] and the Reference Weighted Jaccard (RWJ) [29] metrics quantifies quality based on all generated segments intersecting the reference segment, but have a difference based on the importance (area overlap) of generated segments to the problem. The optimal result for all metrics is zero, with the effective range being [0,1] (with few exceptions). The symbol $n$ denotes the number of generated segments intersecting the given reference segment, while $i$ and $j$ are iterators running through these segments.

Table 5.1. The four spatial empirical discrepancy metrics used for segment evaluation, written using set theory notation.

\begin{tabular}{ccc}
\hline Metric & Formulation & Reference \\
\hline RBSB & $\frac{|R \cup S|-|R \cap S|}{|R|}$ & [27] \\
\hline LSB & $\frac{\left|R \cup S_{h}\right|-\left|R \cap S_{h}\right|+b}{|R|}$ & {$[63]$} \\
\hline PD_OCE & $\sum_{i=1}^{n} \frac{\left|R \cap S_{i}\right|}{\left|R \cup S_{i}\right|} \times \frac{\left|S_{i}\right|}{\sum_{j=1}^{n}\left|S_{j}\right|}$ & {$[64]$} \\
\hline RWJ & $\sum_{i=1}^{n} \frac{\left|R \cap S_{i}\right|}{\left|R \cup S_{i}\right|} \times \frac{\left|R \cap S_{i}\right|}{|R|}$ & {$[29]$} \\
\hline
\end{tabular}

Regarding the optimizers, the "DE/rand/1/bin" variant of the Differential Evolution (DE) [65] metaheuristic is used in this work, based on preliminary experimentation [66] evaluating performances and other published results $[29,43]$ (30 Agents, $\mathrm{F}=0.75, \mathrm{CR}=0.3$, random positions for initialization). For evaluative purposes, Particle Swarm Optimization (PSO) [67] (30 Agents, Inertia Weight = 0.7, 
Best Own $=1.5$, Best Swarm $=1.5)$, a Hill Climber $(\mathrm{HC})(\mathrm{D}=30)$ and random sampling $(\mathrm{RND})$ search are also considered [68] in some experiments.

\subsection{Data}

The proposed approach is demonstrated and evaluated by generating segments on three VHR optical images. The images depict towns and refugee camps in central and east Africa. The images all contain a single thematic class-of-interest with the elements having varying degrees of thematic and spectral similarities, thus presenting the method with a range of problems in terms of difficulty. The aim is to generate a single segment layer, thematically accurate with respect to the land cover elements of interest. Practically, if segment results are adequate, they may be used as is. Otherwise, it may be considered as an initial segmentation, where additional image processing may be needed (e.g., [14]).

The datasets are named after the settlement of interest in the image. The imagery was fully pre-processed (orthorectified, pansharpened), stretched to 8-bit quantization and subsets were extracted over parts of the settlements. For each site twenty elements are digitized, used as the reference segments. Table 5.2 lists the metadata and some usage considerations of the three datasets.

Table 5.2. Metadata of the datasets used.

\begin{tabular}{ccccccc}
\hline Test Site & Target Elements & Sensor & Spatial Resolution & Reference Segments & Channels & Date Captured \\
\hline Bokolmanyo $^{1}$ & Tents & GeoEye-1 & $0.5 \mathrm{~m}$ & 20 & $1,2,3$ & $2011 / 08 / 24$ \\
Jowhaar $^{1}$ & Structures & GeoEye-1 & $1 \mathrm{~m} *$ & 20 & $1,2,3$ & $2011 / 02 / 26$ \\
Hagadera $^{2}$ & Structures & WorldView-2 & $0.75 \mathrm{~m}{ }^{*}$ & 20 & $4,6,3$ & $2010 / 10 / 07$ \\
\hline
\end{tabular}

* Resampled from a $0.5 \mathrm{~m}$ spatial resolution. ${ }^{1}$ (C) GeoEye, Inc. Herndon, VA, USA, 2011, provided by e-GEOS S.p.A. under GSC-DA, all rights reserved. ${ }^{2}$ (C) DigitalGlobe, Inc. Longmont, CO, USA, 2010, provided by EUSI under EC/ESA/GSC-DA, all rights reserved.

Figure 5.8. The datasets used for method evaluation, namely (a) Bokolmanyo; (b) Jowhaar and (c) Hagadera. The blue polygons in the enlarged subsets represent digitized reference segments.
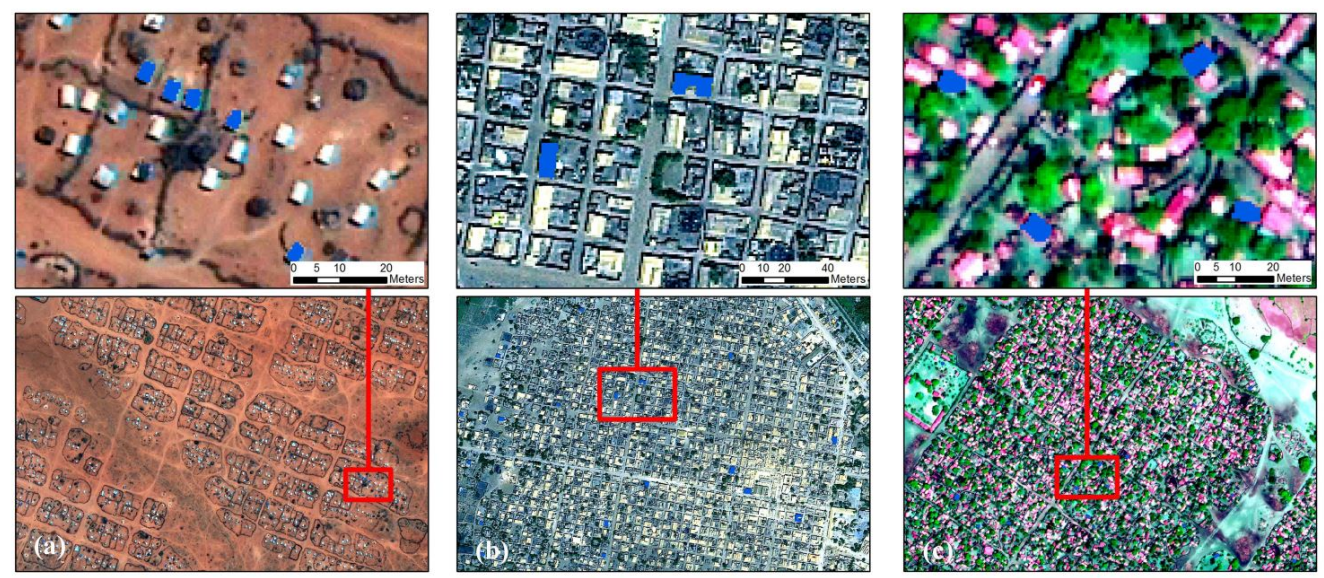
Figure 5.8 shows the three image datasets, or problem instances, along with an enlargement over a small area illustrating the characteristics of the elements of interest. The Bokolmanyo site (a) constitutes an easier problem, where the elements of interest are nylon tents in a refugee camp. A thematic segmentation using the SLIC algorithm would be the aim using this image. The other two datasets, Jowhaar (b) and Hagadera (c), contain more divergence in spectral and thematic correlations of the elements of interest. The MS algorithm is used on these datasets. The aim on these two datasets would be to correctly segment all corrugated iron/steel roofed buildings.

\subsection{Experimental Design}

The described method (Figure 5.3) variants are evaluated based on performances compared to the generic formulation (Figure 5.1) of sample supervised segment generation. Various behavioral characteristics of the method are also quantified, related to the search progression, the feasibility of using different search methods and parameter domain interdependencies. Thus, a comparative experimentalism $[66,69]$ is performed on problem specific datasets.

Due to uncertainty or randomness in terms of sampling (randomly initiated linked list) and classification, metaheuristic progression (initialization, stochastic nature) and segmentation algorithm seeding, multiple runs for experiments are advocated. Results are not specific and have some variation. None the less, in initial experimentation the variance of distributions of results are similar to other work in the context of enlarged search landscapes [29], with statistically significantly different (student's $t$-test and Friedman rank test with Nemenyi post hoc test) results observed on relatively small and large preliminary experimental test sets.

\subsubsection{Segment Quality Comparison and Method Ranking}

The generic formulation of sample supervised segment generation, the proposed variant using probability images for segmentation and three variants conducting image hybridization, namely Hybrid:EB, Hybrid:MA and Hybrid:CP, are quantitatively compared in terms of resultant segment quality. For each test site (Bokolmanyo, Jowhaar and Hagadera) all the method variants are run using the 20 provided reference segments and selected segmentation algorithm (SLIC for Bokolmanyo and MS for Jowhaar and Hagadera). Experimentation is conducted with the four metrics listed in Table 5.1. In total, results are reported with 60 different experimental instances (combinations of methods, problem instances and metrics).

Experimental instances are repeated ten times with the averages, standard deviations and best results achieved reported. Each run consists of 2000 search method iterations using the DE metaheuristic, evaluating the 20 reference structures and taking the mean as the result. In total 24 million segmentation evaluations are performed over the 60 experimental instances. In addition to reporting and discussing the tabularized results, a Friedman test is conducted with a Nemenyi post hoc test [70] to rank the methods and describe their critical differences under all metric and problem type conditions. This is done to give some measure of generalizability [71,72] (commonly done when evaluating multiple classifiers over multiple problem instances), although problem instances and experimental variations are not exhaustive.

\subsubsection{Search Process Characteristics}


The search process is profiled over the three problem instances by recording the fitness traces over all method variants. It is investigated if the higher dimensional formulations of method variants have any significantly different search profiles. Such search-based methods should terminate as quickly as possible (computationally expensive), thus insight in the search progression is beneficial. For each problem instance a metric is selected, RWJ for Bokolmanyo, LSB for Jowhaar and PD_OCE for Hagadera, and the best metric scores (fitness) are plotted at each of the 2000 method iterations. This diversity in experimentation is introduced as a specific metric or segmentation algorithm (search landscape) might generate bias for a specific hybridization strategy.

The fitness profiles are supplemented with a profiling of the required computing time of single fitness evaluations or iterations of the search process. This gives an indication, in the investigated problem contexts and utilized computer (Intel Xeon E5-2643 3.5 GHz processor with single core processing), of the required computing time to achieve optimal or near optimal results. For the Bokolmanyo and Jowhaar sites, average required computing time per evaluation is recorded and averaged over 100 iterations for all five method variants. Results are plotted against optimal achieved metric scores (2000 iterations) of the RWJ metric.

\subsubsection{Parameter Interdependencies}

It is investigated if parameter domain interdependencies exist between the segmentation and sampling/data hybridization components of the, probability, Hybrid:EB, Hybrid:MA and Hybrid:CP method variants. The utilized segmentation algorithms observe spectral aspects as merging criteria (strongly). Any process performing a modification of the spectral characteristics of the data on which the segmentation algorithms run, will inherently influence the optimal values of the segmentation algorithms' parameters. Such interdependency requires the simultaneous optimization of data modification and segmentation algorithm parameters, thus leading to optimization problems with enlarged search spaces as opposed to separately solvable problems.

The optimally achieved segmentation algorithm parameters, sampling and classification parameters and the probability weighting parameter are recorded for experimental runs (2000 search iterations, averaged over ten runs) using the five method variants on the three problem instances considering the RWJ metric. The resultant sensitive "scale" parameters of the SLIC and MS segmentation algorithms, considering the proposed method variants, are specifically compared to the resultant parameters considering the generic variant of the method. A student's $t$-test is performed to determine if differences are present. Three select two dimensional search surface combinations are also plotted (exhaustive fitness calculations) to visually check for, and demonstrate parameter interdependencies, specifically how the MS scale parameter interact with other parameters of the Hybrid:CP method variant in the Jowhaar problem instance.

\subsubsection{Metaheuristic Viability}

Finally the value of using metaheuristics, as opposed to simpler search strategies, is investigated. Simpler search strategies are easier to implement and requires less tuning and would be preferred if metaheuristics provide little performance benefit. It is also investigated how the problem dimensionality (different method variants) affects different search strategies. For one problem instance (Hagadera, MS, RWJ, 2000 iterations, averaged over ten runs) each method variant is run using four different search methods, namely DE, PSO, HC and RND under identical conditions (Section 5.3.6). 
The metaparameters for DE and PSO were hand-tuned (meta-optimization could be considered). Fitness traces are plotted to contrast search progress and general performances under these different search landscape conditions. Final optimal achieved metric scores are reported.

\subsection{Results and Discussions}

\subsubsection{Segment Quality Comparison and Method Ranking}

Tables 5.3-5 lists the results of the metric scores achieved for the three problem instances. The averages and standard deviations are listed. The columns depict method variants and the rows the utilized metrics. Shaded cells highlight the best performing method variants under each metric condition.

Table 5.3. Segmentation accuracies achieved in the Bokolmanyo problem instance. The shaded cells highlight the results of the best performing hybridization strategy.

\begin{tabular}{ccccccc}
\hline Boko (SLIC) & & Generic & Probability & Hybrid:EB & Hybrid:MA & Hybrid:CP \\
\hline \multirow{2}{*}{ LSB } & Average & $0.544 \pm 0.007$ & $0.499 \pm 0.036$ & $0.518 \pm 0.038$ & $0.482 \pm 0.032$ & $0.500 \pm 0.075$ \\
& Best & 0.536 & 0.416 & 0.480 & 0.454 & 0.426 \\
\hline \multirow{2}{*}{ PD_OCE } & Average & $0.718 \pm 0.000$ & $0.445 \pm 0.094$ & $0.538 \pm 0.029$ & $0.358 \pm 0.038$ & $0.371 \pm 0.038$ \\
& Best & 0.718 & 0.367 & 0.486 & 0.309 & 0.318 \\
\hline \multirow{2}{*}{ RBSB } & Average & $0.177 \pm 0.008$ & $0.241 \pm 0.101$ & $0.189 \pm 0.057$ & $0.227 \pm 0.044$ & $0.193 \pm 0.033$ \\
& Best & 0.175 & 0.140 & 0.145 & 0.170 & 0.163 \\
\hline \multirow{2}{*}{ RWJ } & Average & $0.285 \pm 0.006$ & $0.192 \pm 0.012$ & $0.204 \pm 0.019$ & $0.203 \pm 0.017$ & $0.192 \pm 0.012$ \\
& Best & 0.280 & 0.181 & 0.190 & 0.183 & 0.171 \\
\hline
\end{tabular}

Table 5.4. Segmentation accuracies achieved in the Jowhaar problem instance. The shaded cells highlight the results of the best performing hybridization strategy.

\begin{tabular}{ccccccc}
\hline Jowhaar (MS) & & Generic & Probability & Hybrid:EB & Hybrid:MA & Hybrid:CP \\
\hline \multirow{2}{*}{ LSB } & Average & $0.371 \pm 0.006$ & $0.302 \pm 0.008$ & $0.299 \pm 0.007$ & $0.313 \pm 0.005$ & $0.296 \pm 0.014$ \\
& Best & 0.361 & 0.295 & 0.285 & 0.307 & 0.281 \\
\hline \multirow{2}{*}{ PD_OCE } & Average & $0.581 \pm 0.010$ & $0.413 \pm 0.034$ & $0.434 \pm 0.024$ & $0.361 \pm 0.034$ & $0.315 \pm 0.040$ \\
& Best & 0.558 & 0.382 & 0.409 & 0.301 & 0.261 \\
\hline \multirow{2}{*}{ RBSB } & Average & $0.187 \pm 0.008$ & $0.223 \pm 0.091$ & $0.184 \pm 0.045$ & $0.207 \pm 0.018$ & $0.201 \pm 0.030$ \\
& Best & 0.180 & 0.118 & 0.141 & 0.182 & 0.170 \\
\hline \multirow{2}{*}{ RWJ } & Average & $0.230 \pm 0.004$ & $0.182 \pm 0.032$ & $0.175 \pm 0.018$ & $0.233 \pm 0.037$ & $0.196 \pm 0.029$ \\
& Best & 0.225 & 0.145 & 0.138 & 0.182 & 0.159 \\
\hline
\end{tabular}

Table 5.5. Segmentation accuracies achieved in the Hagadera problem instance. The shaded cells highlight the results of the best performing hybridization strategy.

\begin{tabular}{ccccccc}
\hline Hagadera (MS) & & Generic & Probability & Hybrid:EB & Hybrid:MA & Hybrid:CP \\
\hline \multirow{2}{*}{ LSB } & Average & $0.628 \pm 0.005$ & $0.564 \pm 0.030$ & $0.549 \pm 0.019$ & $0.525 \pm 0.034$ & $0.537 \pm 0.018$ \\
& Best & 0.622 & 0.532 & 0.513 & 0.488 & 0.512 \\
\hline \multirow{2}{*}{ PD_OCE } & Average & $0.692 \pm 0.028$ & $0.474 \pm 0.009$ & $0.540 \pm 0.027$ & $0.464 \pm 0.014$ & $0.464 \pm 0.017$ \\
& Best & 0.681 & 0.464 & 0.501 & 0.445 & 0.427 \\
\hline \multirow{2}{*}{ RBSB } & Average & $0.371 \pm 0.010$ & $0.406 \pm 0.022$ & $0.330 \pm 0.029$ & $0.340 \pm 0.057$ & $0.387 \pm 0.079$
\end{tabular}




\begin{tabular}{ccccccc} 
& Best & 0.350 & 0.383 & 0.276 & 0.274 & 0.272 \\
\hline \multirow{2}{*}{ RWJ } & Average & $0.437 \pm 0.007$ & $0.314 \pm 0.019$ & $0.313 \pm 0.028$ & $0.339 \pm 0.029$ & $0.326 \pm 0.026$ \\
& Best & 0.424 & 0.284 & 0.286 & 0.301 & 0.293 \\
\hline
\end{tabular}

Firstly, variation is noted in which method variants perform best under different metric conditions, justifying an investigation using multiple metrics. The use of a single quality metric would create bias. The magnitude of differences also varies, depending on the metric used and the problem instance. Very small standard deviations in results are observed when considering the generic formulation of the method, with the variants consisting of enlarged search spaces and containing more stochastic processes displaying larger variation in optimally achieved results. The intensities of the variations are also metric dependent, with the RBSB metric showing the most variation in results. It is conjectured that the search surfaces resultant from the RBSB metric (Table 5.1) creates more irregularities/noise, due to its simpler formulation that only considers a generated segment with the largest overlap with a reference segment.

Considering Table 5.3, depicting the Bokolmanyo problem instance using the SLIC segmentation algorithm, the Hybrid:MA strategy provided the best segment results when considering the LSB and PD_OCE metrics. The Hybrid:CP strategy performed best when considering the RWJ metric and the generic method variant when considering the RBSB metric. Comparing the generic variant with the proposed variants, substantial improvements in quality is observed under LSB, PD_OCE and RWJ metric conditions.

For the Jowhaar problem instance (Table 5.4) using the MS segmentation algorithm, the Hybrid:CP (LSB and PD_OCE) and Hybrid:EB (RBSB and RWJ) strategies provided the best results. Again, significant improvements in results are achieved compared to the generic formulation of the method, with results with the RBSB metric the exception. Figure 5.9 illustrates segmentation results obtained, focused on a single reference structure chosen randomly, under the RWJ metric condition. Note that results are averaged over all provided reference segments, and that the illustrated RWJ scores are segment specific.

Similarly on the Hagadera test site (Table 5.5), constituting the most difficult problem, the Hybrid:EB, Hybrid:MA and Hybrid:CP method variants produced the best results. Results in this problem instance was generally worse than on the other two datasets, strongly suggesting the use of additional image processing to further process segments. Notwithstanding, significant improvements in results from the hybrid method variants are still useful (much closer to correct). Generally speaking, the proposed method variants have less specific results (more uncertainty) due to the addition of more free parameters and classification processes, but still perform much better than the generic variant in many instances.

Figure 5.10 illustrates a Nemenyi post hoc test conducted after a Friedman test, ranking the performances of the five investigated method variants based on the 12 experiments (three problem instances and four metrics) conducted with each. The Hybrid:CP strategy performed the best, followed by the other hybridization strategies. The generic formulation of the method (Original Image) is ranked the lowest. A critical difference exist between the Hybrid:CP and the generic method. If the RBSB metric (problematic) is omitted from the test, illustrated in Figure 5.11, all method variants perform better with a critical difference compared to the generic method. Thus, even with the variation introduced by the metaheuristic and classification, the methods are still useful. The Hybrid:CP strategy 
is still ranked first. Critical differences are not present among proposed method variants, suggesting a strong dependence on problem conditions and the nature of the metric and the optimal hybridization strategy. Interestingly, segmenting with the probability image is ranked better than the Hybrid:EB strategy. This could be different if a different band was replaced or if the probability image was merely added to the image stack.

Figure 5.9. Segmentation results achieved with the Jowhaar problem instance (MS) using the RWJ metric. The red polyline delineate one of the twenty reference segments (a) provided by a user; (b) Shows results with the generic formulation; (c) using the probability image and the (d) Hybrid:EB; (e) Hybrid:MA and (f) Hybrid:CP strategies. RWJ metric scores for this specific structure are also given.

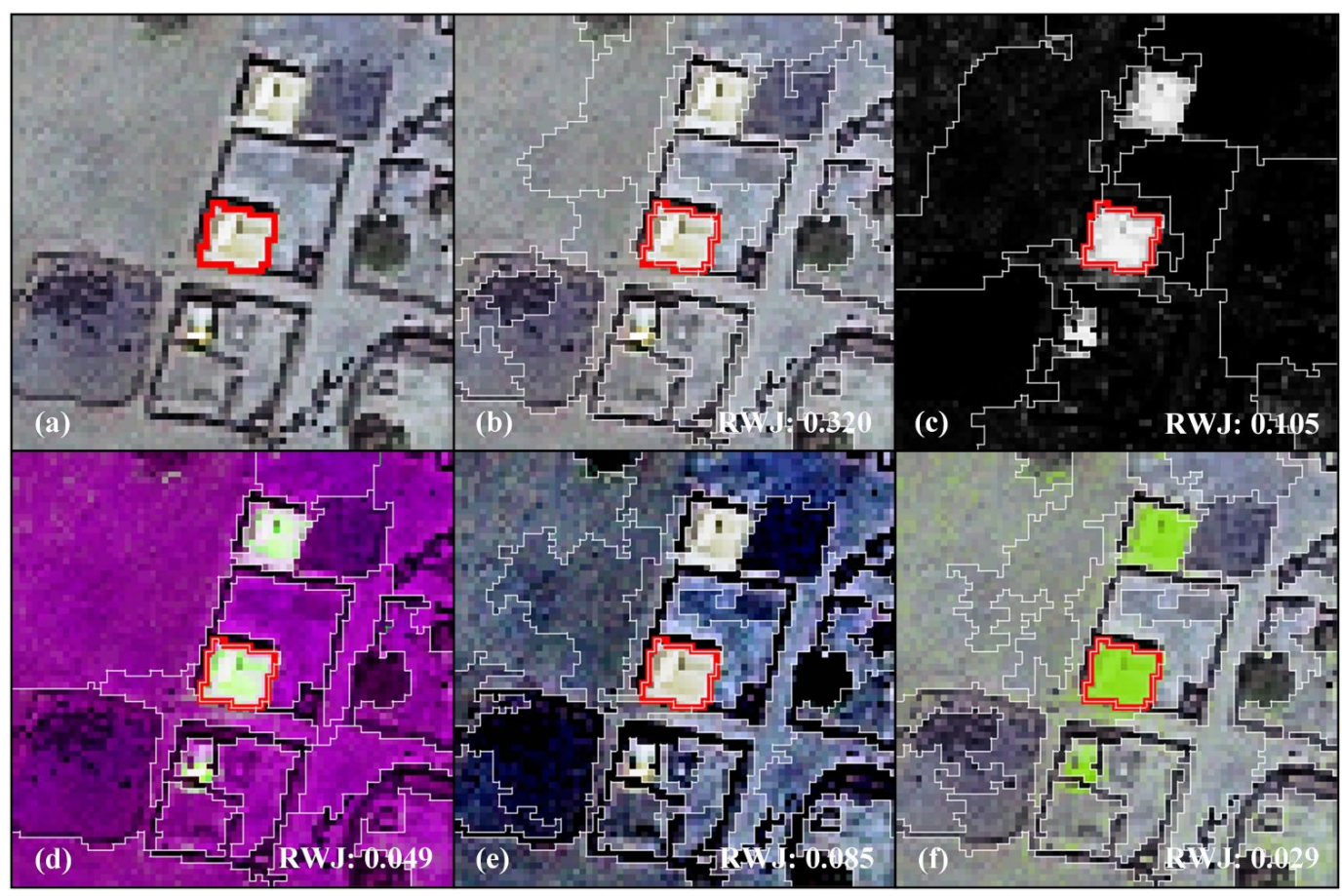

Figure 5.10. Nemenyi post hoc test performed after a Friedman test, ranking the different methods over all metric and problem instances. Horizontal lines indicate the Critical Differences (CD).

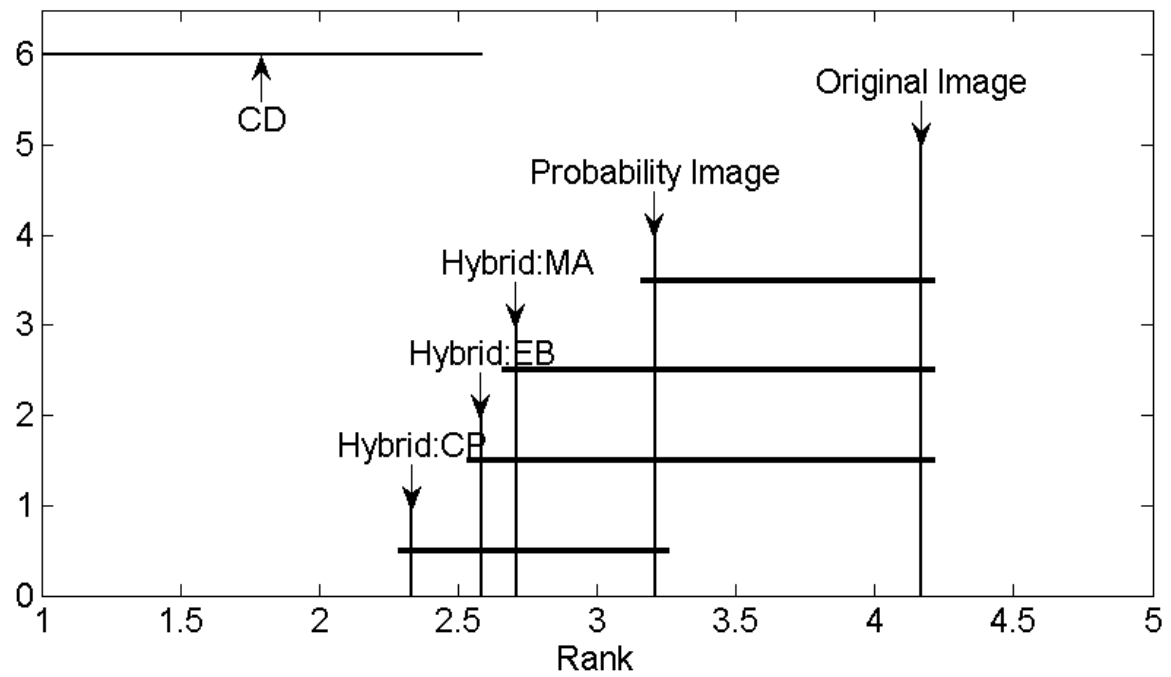


Figure 5.11.The Nemenyi post hoc test, with the RBSB metric omitted. Horizontal lines indicate the Critical Differences (CD).

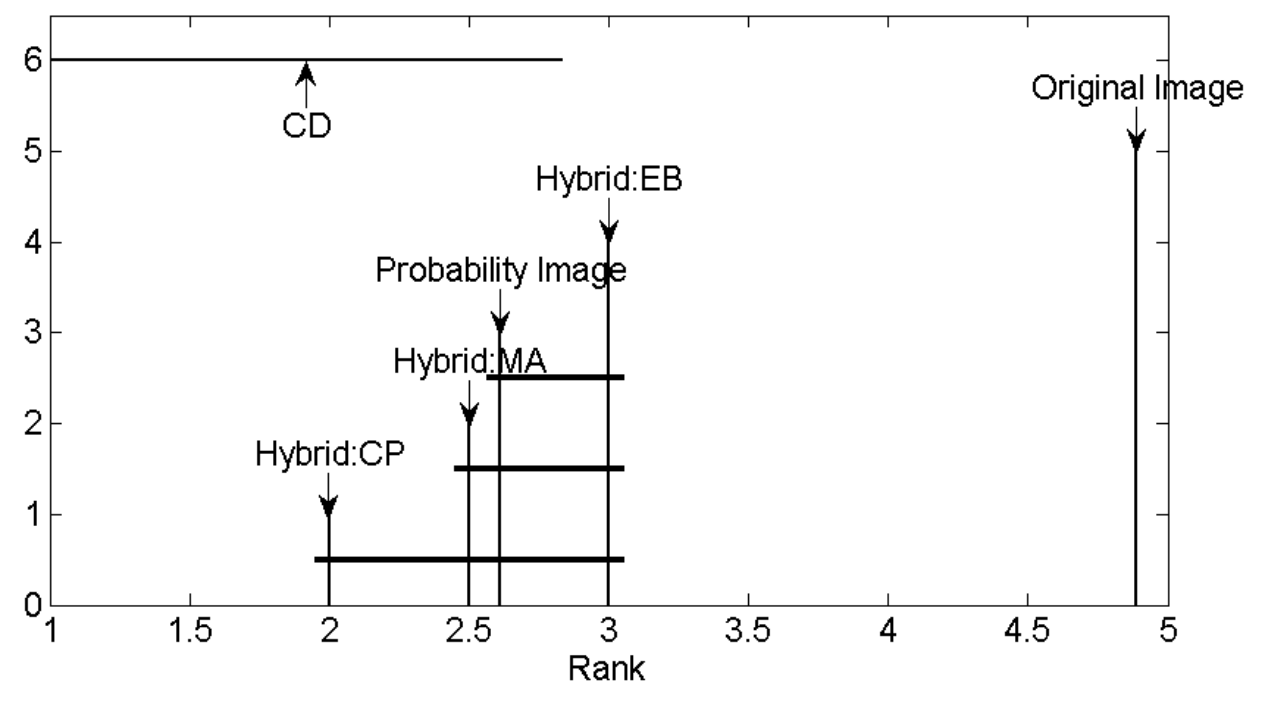

\subsubsection{Search Process Characteristics}

Figures 5.12-14 illustrate the fitness traces over the three problem instances (averaged over ten runs), specifically, Figure 5.12 show the fitness traces for the Bokolmanyo problem instance using the RWJ metric, Figure 5.13 the fitness traces for the Jowhaar problem instance using the LSB metric and Figure 5.14 the fitness traces for the Hagadera problem instance with the PD_OCE metric.

In all of the problem instances the proposed method variants obtain better results very early on in the search process compared to the generic variant of the method. This can be attributed to the nature of the probability image, which in many cases immediately provides improved results irrespective of how the parameters of the segmentation algorithm are tuned or how the hybridization parameters are tuned.

Observing Figure 5.12, the low dimensional generic variant of the method (only two dimensions of the parameters of the SLIC segmentation algorithm), obtains its best fitness extremely early on in the search process, at around 200 fitness evaluations. All other method variants already provide better fitness at this mark, with optimal results achieved near the 1000 iteration mark. Thus, the simpler, lower dimensional method variants have no initial advantage over the more elaborate method variants, suggesting no penalties in terms of search iterations. In this specific instance, only the probability image variant lags slightly behind the generic variant.

Figures 5.13 and 5.14 (MS segmentation) have similar profiles, with the proposed method variants outperforming the generic variant in terms of fitness throughout the search process. Interestingly, all four variants have very similar fitness profiles, with the exception of the Hybrid:MA strategy on the Hagadera problem instance. These results suggest the proposed method variants obtain better results faster, even though their search landscape dimensionalities are higher. Under these experimental conditions, 1000 search iterations seem sufficient for the methods to obtain their optimal results, which parallels with results reported elsewhere [29,43], even though search landscapes may have different characteristics. 
Figure 5.12. Fitness traces for the Bokolmanyo problem instance.

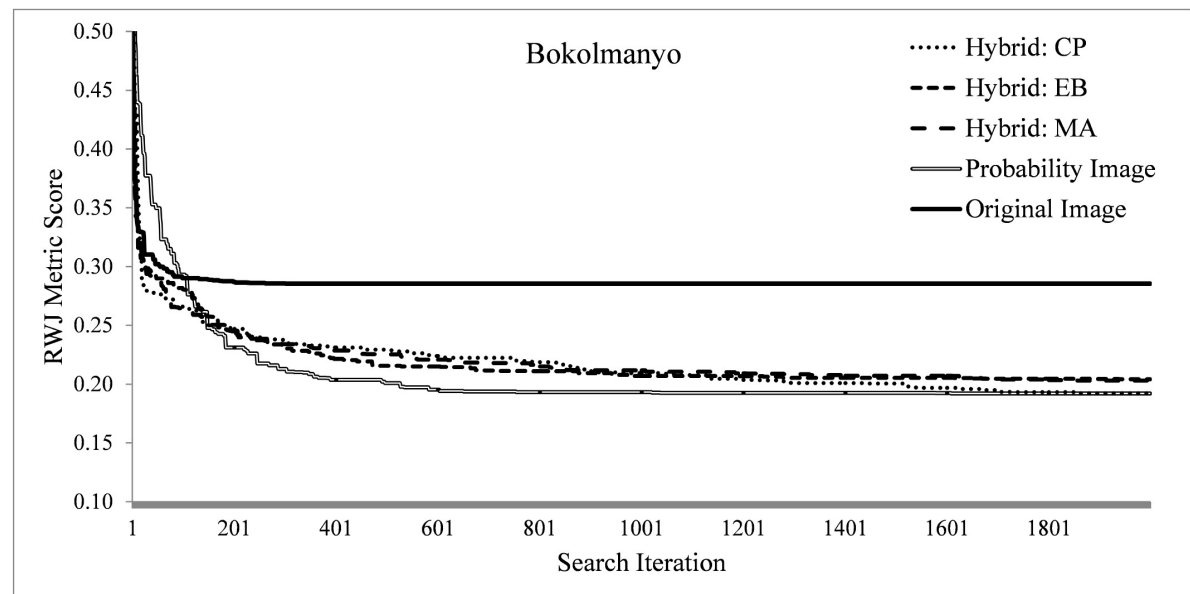

Figure 5.13. Fitness traces for the Jowhaar problem instance.

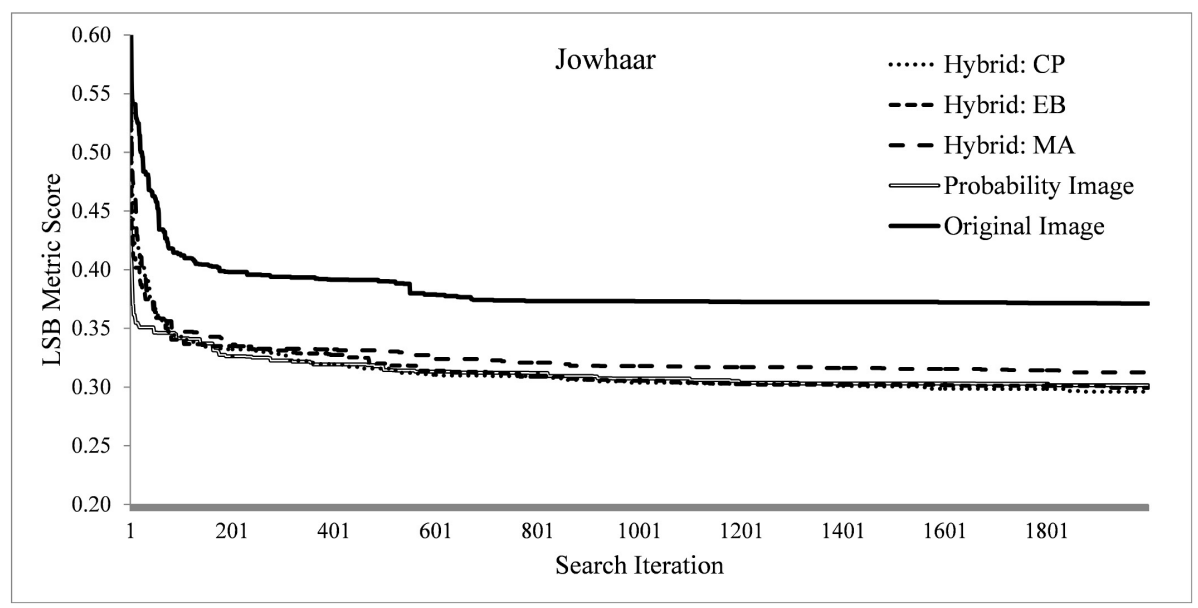

Figure 5.14. Fitness traces for the Hagadera problem instance.

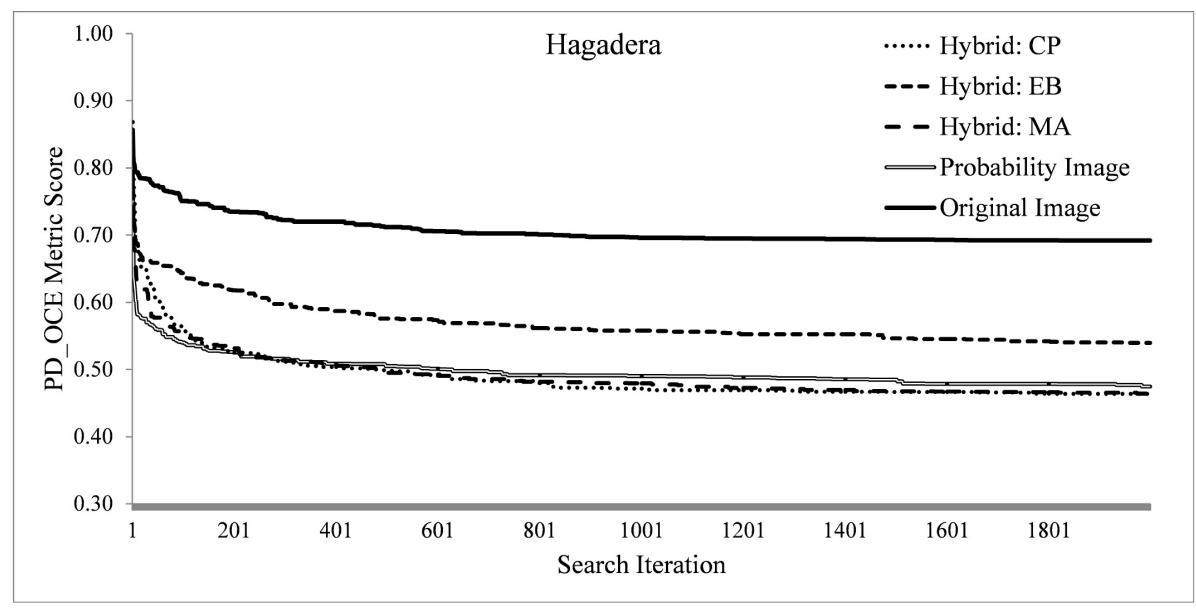

Figures 5.15 and 5.16 illustrate the search iteration execution times for the Bokolmanyo (Figure 5.15) and Jowhaar (Figure 5.16) problem instances. A single search method evaluation instance (fitness evaluation) encompasses segmenting 20 image subsets (around the reference segments) and comparing the generated segments with that of the reference segment using a given metric. With the proposed method 
variants, sampling, classification and image hybridization processes are added. Results are averaged over 100 runs.

Figure 5.15. Search iteration execution time profiling for the Bokolmanyo problem instance.

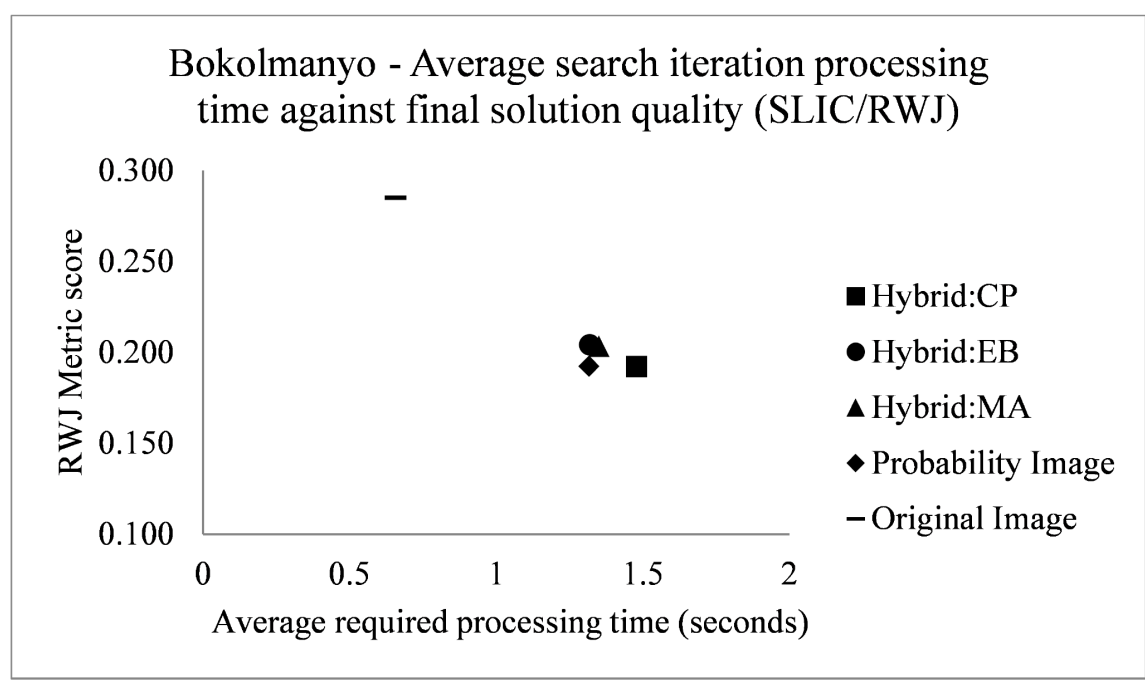

The original variant of the method takes around $0.5 \mathrm{~s}$ to complete a single evaluation in both problem instances. In the context of the Bokolmanyo problem instance the proposed method variants needs about three times longer to execute. The majority of the time is taken by the masking process (computational profiling), specifically the libSVM [73] predict function and to a lesser extent the two-class probability image classification process. As the number of support vectors increase, so do the required computing time to determine the label of a new pixel under consideration.

Figure 5.16. Search iteration execution time profiling for the Jowhaar problem instance.

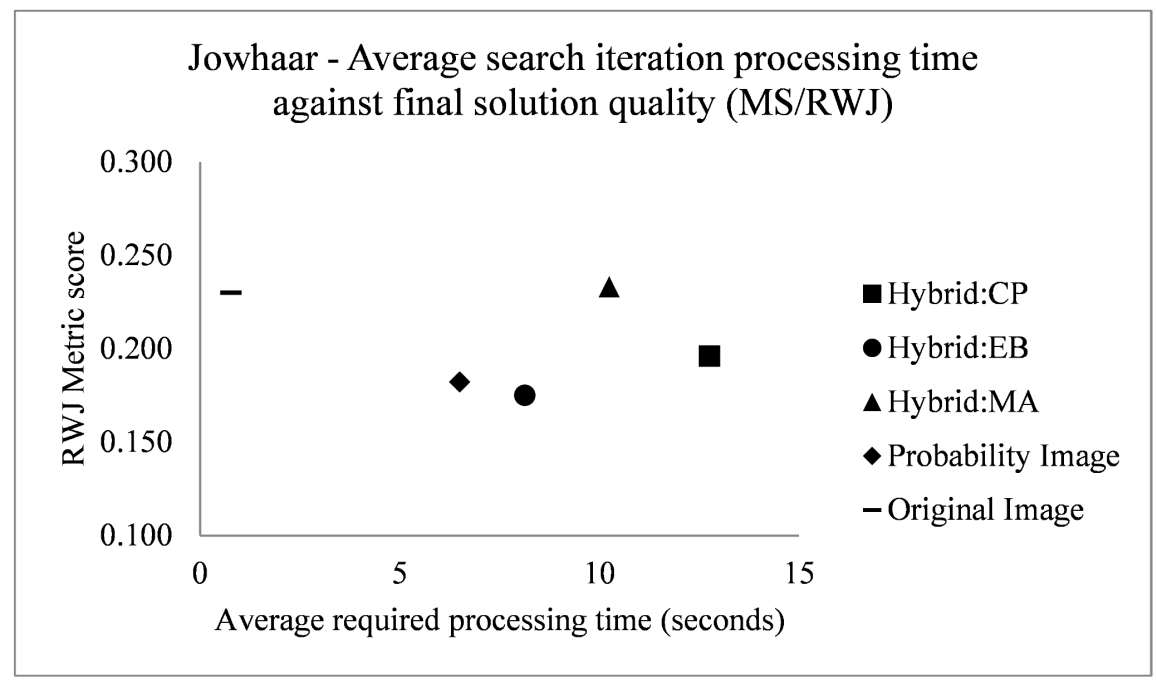

Figure 5.16 shows a similar characteristic, but with lengthier execution times and more variation depending on the method variant used. Such variation can be explained by the nature of the probability image that was found most useful, having varying numbers of support vectors (and thus increasing 
the time required to predict pixel values). Alternative classifiers, which predict faster, may be considered to alleviate the increase in required computing times.

\subsubsection{Parameter Interdependencies}

Tables 5.6-8 list the average parameter values obtained for the three problem instances. The columns list the segmentation algorithm and probability image generation parameters. "S nu" and "S gamma" are the one-class SVM parameters and "C C" and "C Gamma" the two-class SVM parameters.

The rows delineate the method variant under consideration. The shaded cells indicate scale parameters achieved under proposed method variant conditions, which differ from the resultant scale parameter under the generic method variant condition (student's $t$-test with a 95\% confidence interval). In all but one of the problem instances, with both the SLIC and MS segmentation algorithms, the proposed method variants have statistically significantly different optimal scale parameters compared to the generic method variant. This suggest an influence of probability and hybridization (where applicable) processes on the optimal scale parameter within the given segmentation algorithm.

In the case of the SLIC algorithm (Table 5.6), very specific optimal scale parameters are generated. In the case of the MS algorithm (Tables 5.7 and 5.8), more diversity is present, with differences (optimal values and standard deviations) attributed to the natures of the different method variants. All the other parameters illustrate extreme variation in optimal results obtained, suggesting multiple combinations of parameters can deliver optimal or near optimal results (within the capability of the method-thus multiple "near global optima" in search landscape terms). This corroborates the importance of the scale parameters with these two segmentation algorithms. The probability image is very beneficial in most cases, with the weighting parameter having relatively high values in most instances.

Table 5.6. Parameters generated for the Bokolmanyo problem instance.

\begin{tabular}{cccccccc}
\hline SLIC/RWJ & Scale & Compactness & S Nu & S Gamma & C C & C Gamma & Weight \\
\hline Original & $9.500 \pm 0.745$ & $20.710 \pm 3.653$ & NA & NA & NA & NA & NA \\
Probability & $8.100 \pm 1.449$ & $22.360 \pm 2.723$ & $0.077 \pm 0.086$ & $64.400 \pm 34.004$ & $0.002 \pm 0.000$ & $48.200 \pm 36.715$ & NA \\
Hybrid:EB & $9.150 \pm 0.530$ & $19.180 \pm 4.193$ & $0.099 \pm 0.077$ & $27.600 \pm 38.295$ & $9.800 \pm 30.990$ & $47.800 \pm 40.524$ & NA \\
Hybrid:MA & $8.100 \pm 2.196$ & $19.120 \pm 4.994$ & $0.079 \pm 0.090$ & $43.800 \pm 37.434$ & $26.600 \pm 37.441$ & $47.800 \pm 27.848$ & $0.730 \pm 0.239$ \\
Hybrid:CP & $8.700 \pm 1.457$ & $23.290 \pm 2.388$ & $0.065 \pm 0.086$ & $48.800 \pm 35.916$ & $39.600 \pm 39.764$ & $52.600 \pm 33.586$ & $0.664 \pm 0.151$ \\
\hline
\end{tabular}

Table 5.7. Parameters generated for the Hagadera problem instance.

\begin{tabular}{ccccccccc}
\hline MS/RWJ & Scale & Colour & Compt & S Nu & S Gamma & C C & C Gamma & Weight \\
\hline \multirow{2}{*}{ Original } & 17.430 & 0.132 & 0.514 & & & & & \\
& \pm 8.200 & \pm 0.110 & \pm 0.389 & NA & NA & NA & NA & NA \\
& 5.990 & 0.074 & 0.481 & 0.060 & 40.400 & 34.600 & 29.400 & NA \\
Probability & \pm 1.211 & \pm 0.081 & \pm 0.302 & \pm 0.079 & \pm 43.090 & \pm 40.189 & \pm 37.131 & \\
& & & & & & & \\
Hybrid:EB & 8.300 & 0.046 & 0.219 & 0.107 & 55.000 & 25.400 & 37.400 & NA \\
& \pm 9.334 & \pm 0.136 & \pm 0.259 & \pm 0.082 & \pm 25.910 & \pm 39.934 & \pm 43.950 & \\
\hline
\end{tabular}




\begin{tabular}{|c|c|c|c|c|c|c|c|c|}
\hline \multirow{2}{*}{ Hybrid:MA } & 9.070 & 0.068 & 0.263 & 0.127 & 59.000 & 22.600 & 49.600 & 0.772 \\
\hline & \pm 10.640 & \pm 0.171 & \pm 0.253 & \pm 0.087 & \pm 35.380 & \pm 28.316 & \pm 34.329 & \pm 0.180 \\
\hline \multirow{2}{*}{ Hybrid:CP } & 5.880 & 0.036 & 0.442 & 0.072 & 65.200 & 28.200 & 60.600 & 0.906 \\
\hline & \pm 1.623 & \pm 0.050 & \pm 0.333 & \pm 0.078 & \pm 43.627 & \pm 43.782 & \pm 39.090 & \pm 0.161 \\
\hline
\end{tabular}

Table 5.8. Parameters generated for the Jowhaar problem instance.

\begin{tabular}{|c|c|c|c|c|c|c|c|c|}
\hline MS/RWJ & Scale & Colour & Compt & $\mathbf{S} \mathbf{N u}$ & S Gamma & $\mathbf{C ~ C}$ & C Gamma & Weight \\
\hline Original & $\begin{array}{c}15.890 \\
\pm 3.646\end{array}$ & $\begin{array}{c}0.054 \\
\pm 0.023\end{array}$ & $\begin{array}{c}0.692 \\
\pm 0.245\end{array}$ & NA & NA & NA & NA & NA \\
\hline Probability & $\begin{array}{c}21.317 \\
\pm 3.507\end{array}$ & $\begin{array}{c}0.146 \\
\pm 0.112\end{array}$ & $\begin{array}{c}0.736 \\
\pm 0.225\end{array}$ & $\begin{array}{c}0.081 \\
\pm 0.091\end{array}$ & $\begin{array}{c}22.000 \\
\pm 26.743\end{array}$ & $\begin{array}{c}40.667 \\
\pm 30.820\end{array}$ & $\begin{array}{c}51.667 \\
\pm 23.304\end{array}$ & NA \\
\hline Hybrid:EB & $\begin{array}{c}17.571 \\
\pm 8.253\end{array}$ & $\begin{array}{c}0.049 \\
\pm 0.045\end{array}$ & $\begin{array}{c}0.602 \\
\pm 0.379\end{array}$ & $\begin{array}{c}0.096 \\
\pm 0.101\end{array}$ & $\begin{array}{c}57.143 \\
\pm 46.056\end{array}$ & $\begin{array}{c}76.286 \\
\pm 12.880\end{array}$ & $\begin{array}{c}46.571 \\
\pm 35.132\end{array}$ & NA \\
\hline Hybrid:MA & $\begin{array}{c}27.943 \\
\pm 7.056\end{array}$ & $\begin{array}{c}0.385 \\
\pm 0.245\end{array}$ & $\begin{array}{c}0.762 \\
\pm 0.366\end{array}$ & $\begin{array}{c}0.113 \\
\pm 0.084\end{array}$ & $\begin{array}{c}50.571 \\
\pm 40.078\end{array}$ & $\begin{array}{c}60.000 \\
\pm 31.305\end{array}$ & $\begin{array}{c}44.571 \\
\pm 38.587\end{array}$ & $\begin{array}{c}0.911 \\
\pm 0.046\end{array}$ \\
\hline Hybrid:CP & $\begin{array}{c}33.600 \\
\pm 11.055\end{array}$ & $\begin{array}{c}0.423 \\
\pm 0.293\end{array}$ & $\begin{array}{c}0.747 \\
\pm 0.360\end{array}$ & $\begin{array}{c}0.118 \\
\pm 0.090\end{array}$ & $\begin{array}{r}42.286 \\
\pm 33.950\end{array}$ & $\begin{array}{c}53.429 \\
\pm 28.230\end{array}$ & $\begin{array}{c}20.857 \\
\pm 32.308\end{array}$ & $\begin{array}{c}0.937 \\
\pm 0.068\end{array}$ \\
\hline
\end{tabular}

Figures 5.17-19 illustrate two dimensional search surface slices obtained with the Jowhaar problem instance using the Hybrid:CP method variant. For each parameter combination the fitness over 20 reference segments were calculated and plotted. The metric used was RWJ and the segmentation algorithm was MS. The scale parameter is contrasted with three other parameters, namely the second Central Position parameter (CP2) of the Hybrid:CP variant (Figure 5.17), the $\mathrm{C}$ parameter of the two-class SVM (Figure 5.18) and the probability weighting parameter (Figure 5.19). All other parameters were given initial random values that did not change during the experiment. The depicted search surfaces for these parameter pairings may be completely different under alternative random parameter settings, specifically under alternative "Color/Shape" parameter settings.

Figure 5.17. Search landscape slice with the Scale and CP2 parameter of the Hybrid:CP method variant.

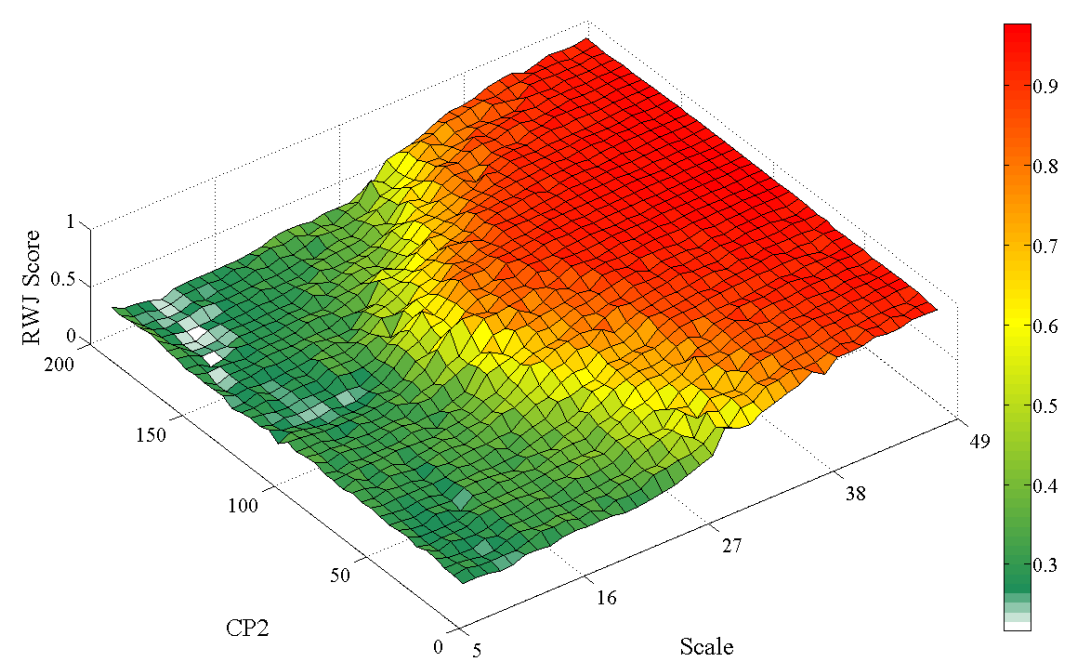


Figures 5.17 and 5.19 visually illustrate parameter interdependencies, as expected, of the Scale and CP2 and Scale and Weight parameters, respectively. The bulge in Figure 5.17 may suggest that attempting to set the CP parameter to 125 (a spectral position) a greater range of scale parameters will result in a merging of the reference segment area with its surroundings. In contrast, Figure 5.18 shows very little influence of the $C$ parameter, a very insensitive parameter in these formulations, on the scale parameter of the segmentation algorithm. Interestingly, and explainable, Figure 5.19 shows a narrower range of scale parameter values applicable as the influence of the probability image in hybridization increases.

Figure 5.18. Search landscape slice with the Scale and two-class SVM C parameter of the Hybrid:CP method variant.

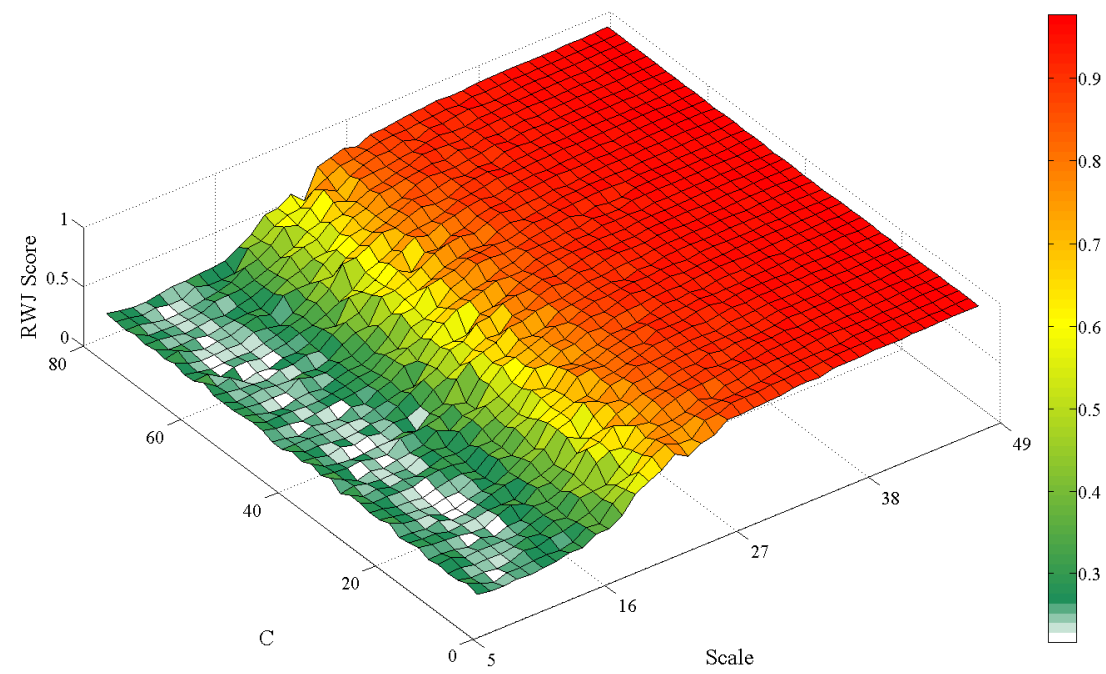

Figure 5.19. Search landscape slice with the Scale and probability weighting parameter of the Hybrid:CP method variant.

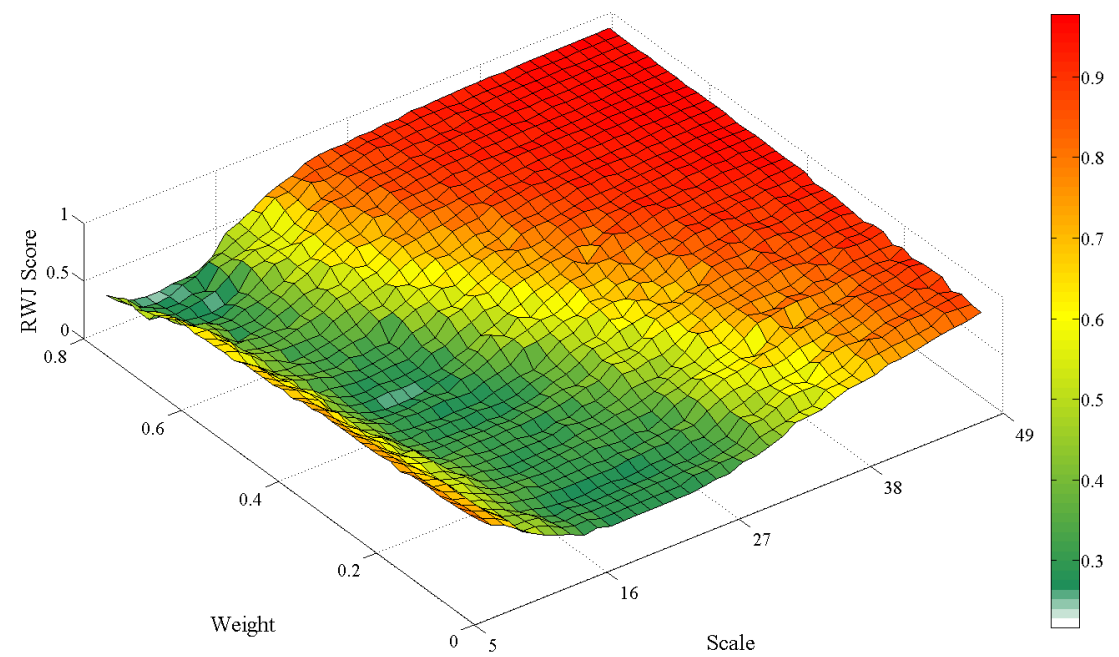

\subsubsection{Metaheuristic Viability}

Figure 5.20 shows fitness traces generated for the Hagadera test site with the five investigated method variants, using four different search methods, namely DE, PSO, HD and RND. The dimensionality of the search problem in the generic method variant is three (Figure 5.20a), seven in the case of using the 
probability image (Figure 5.20b) to segment and with the Hybrid:EB (Figure 5.20c) variant, eight when considering the Hybrid:MA (Figure 5.20d) variant and eleven with the Hybrid:CP (Figure 5.20e) method variant.

Figure 5.20. Fitness traces using different search methods for the Hagadera problem instance. Each subfigure shows results using a different method variant.

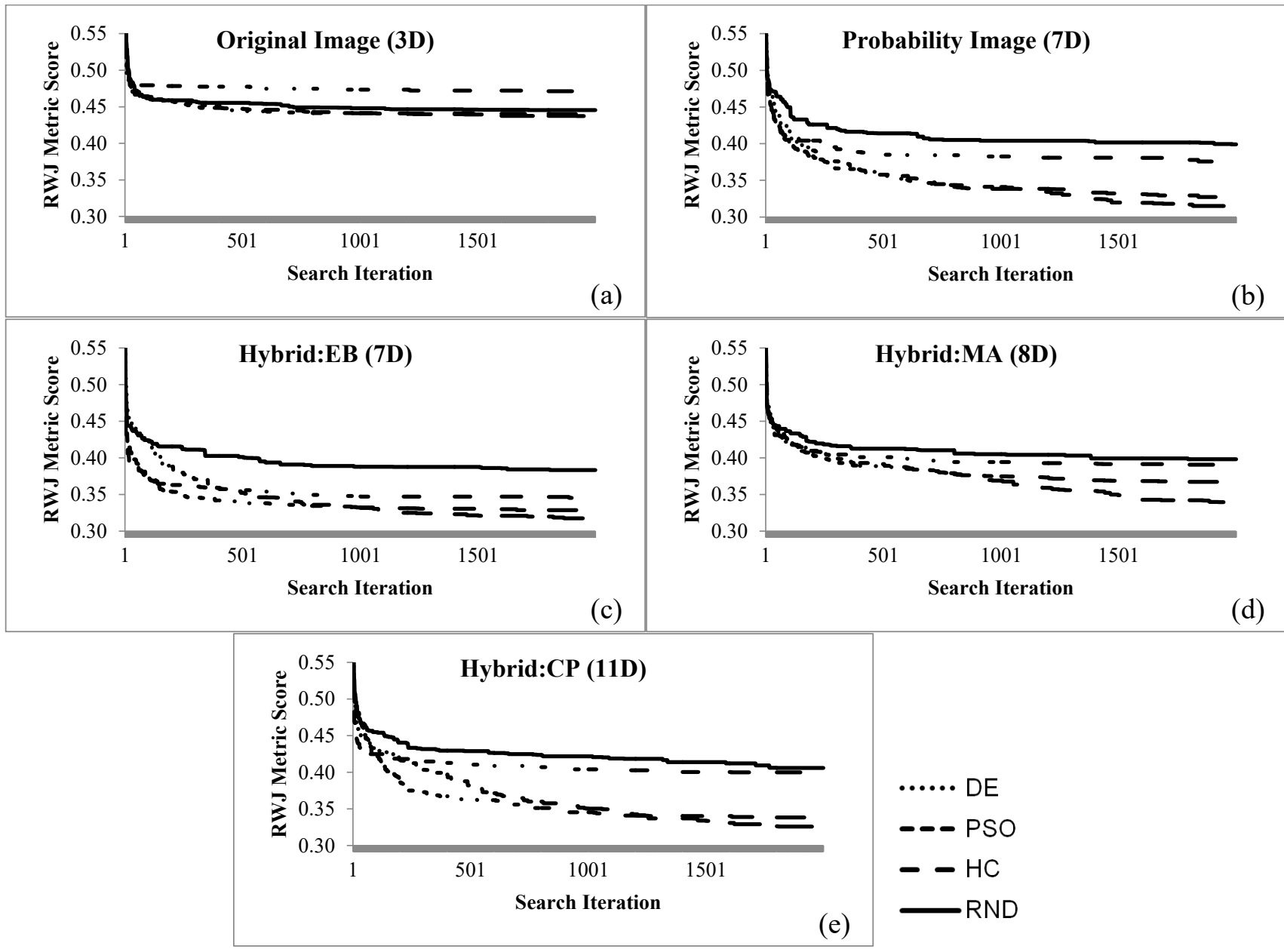

Table 5.9. RWJ metric scores using the four different search strategies in the Hagadera problem instance. The shaded cells highlight the results of the best performing search method.

\begin{tabular}{cccccc}
\hline MS/RWJ & Original & Probability & Hybrid:EB & Hybrid:MA & Hybrid:CP \\
\hline DE & $0.437 \pm 0.007$ & $0.314 \pm 0.019$ & $0.313 \pm 0.028$ & $0.339 \pm 0.029$ & $0.326 \pm 0.026$ \\
PSO & $0.440 \pm 0.016$ & $0.327 \pm 0.041$ & $0.328 \pm 0.029$ & $0.367 \pm 0.047$ & $0.338 \pm 0.042$ \\
HC & $0.470 \pm 0.013$ & $0.372 \pm 0.012$ & $0.345 \pm 0.011$ & $0.391 \pm 0.018$ & $0.400 \pm 0.020$ \\
RND & $0.446 \pm 0.006$ & $0.399 \pm 0.033$ & $0.383 \pm 0.015$ & $0.398 \pm 0.027$ & $0.406 \pm 0.027$ \\
\hline
\end{tabular}

In all instances the DE metaheuristic produced the best resulting segment quality, followed closely by the PSO metaheuristic. DE converges more slowly than PSO, a known characteristic [74]. More importantly, large differences exist between the simpler HC and RND strategies and the DE and PSO strategies on the higher dimensional problems. In the original formulation of the method, little benefit is seen from using the more advanced search strategies. On the larger search landscapes of the proposed method variants more advanced search strategies are certainly required. Table 5.9 lists 
the optimal metric scores achieved in these experimental runs, with the best results per method variant highlighted with shaded cells, reflecting the results illustrated in Figure 5.20.

\subsection{Conclusions}

A major driver behind the more elaborate methods encountered in the context of VHR optical image analysis is the divergence of the spectral and thematic correlations. Such correlations are stronger in lower resolution imagery and problem contexts. A novel method in the context of sample supervised segment generation was presented, where a classification process attempts to tailor the data such that closer thematic and spectral correlations exist. The given segmentation algorithm may thus perform better on the given problem. The method entails the creation of an enlarged search landscape, with added parameters controlling probability image generation and data hybridization method components. These components are tunable and do not deliver static results on their own. Their interaction is also tunable in some variants. Throughout the process, the aim is still quality segment generation for a specific element type and classification accuracy assessment is not conducted.

Four method variants were compared with the generic formulation of sample supervised segment generation in terms of resultant segment quality, illustrating the usefulness of such a method. The magnitude of improvements is dependent on the problem, metric, search method and method variant under consideration. In the current method formulation, a substantial amount of extra computing time is required, majorly due to the internal details of the used classifier (SVM). It should be noted that this impact is more pronounced during the training phase. The use of metaheuristics and the definition of enlarged search spaces were also justified. Although the proposed method variants improve results substantially in many problem instances, "perfect" metric scores were not achieved, suggesting that, as with the generic method formulation, additional image processing may be needed to obtain thematically accurate segments. Such segments may subsequently be classified for map production or information extraction.

Uncertainties exist with a sample supervised segment generation approach in general and the variants presented here in particular. In general, uncertainty remains if a given problem is feasible, and a method needs to be run to verify its applicability, which takes time. Expert knowledge on the characteristics of the used segmentation algorithm may help, but with the variants proposed here unintuitive, but correct or feasible results may also be generated. Some spectral and thematic correlation needs to exist for the elements of interest, not explicitly quantified or investigated in this study. Initial experimentation on synthetic datasets suggest that elements of interest may constitute up to three "regions" in the spectral domain, with more resulting in a significant drop in the usefulness of the generated probability image. Having elements of interest consist of six unique spectral regions (red, blue, green, yellow, cyan, and magenta) on synthetic data generated an almost completely monotone probability image (one-class SVM, RBF kernel). Nonetheless, if not found useful or even if detrimental, the probability image is simply not used (controlled via the weighting parameter). Quantifying the usefulness of such an approach on spectrally diversifying elements of interest would be a topic for future research.

The generalizability of such approaches under different sampling conditions should also be investigated [29]. As a preliminary experiment in this work, sixteen method variants were tested under cross-validated and non-cross-validated sampling conditions (20-28 reference samples), all performing 
better than the generic formulation of the method. For operational use, on such large sample sets cross-validation would not be necessary. This could change with a sharp decrease in the number of samples used, observed in [29]. Indicators of required sampling sizes would be useful and is planned for future work. In addition, a variant of this method is possible that could require a user to provide samples of the "other" class, removing the parameterized process of generating synthetic "other" class samples. This would require additional user interaction outside of the required class of interest.

The accuracy and convergence speed of the methods may be improved by performing meta-optimization, using metaheuristics with self-adapting meta-parameters or pursuing the state of the art in evolutionary computation. Additionally, most aspects of such methods could be designed to run in a parallel framework, especially the computationally expensive fitness evaluations. Sample supervised segment generation may well be integrated with more traditional GEOBIA approaches, such as rule set development, necessitating near real-time method executions. It would also be of interest to compare such an approach based on classifier directed transforms with a strategy that suggest the addition of low-level image processing to modify the data, or so called data transformation functions [29]. Such transformation functions do not have the same computing overhead as some classification processes, but on the other hand they may not be able to achieve the same level of quality as classifier based transforms. Alternative classification algorithms could also be tested with such an approach.

Finally, it should be noted that the method, and its variants presented here have an explicit implementation how sampling and classification is done, e.g., Figure 5.5. Various other encodings of sample collection, probability image generation and image hybridization are possible. A simple extension of the proposed method could see the sampling of the synthetic secondary class (Figure 5.5b) grouped by underlying spectral content, with parameters controlling sub-selections used in classification. This would create more variation in the characteristics of probability image outputs. Also, the image hybridization strategies could be elaborated upon to include thresholds of probability values, to use neighborhood properties or spectral aspects or even to use parameter controlled band selection strategies. Additionally, more segmentation algorithms could be tested with this method.

Search landscape characteristics should always be kept in mind, as noise or too much randomization introduced by parameters controlling elaborate processes may create more "difficult" search landscapes. Figures 5.17-19 illustrate smooth or, conjectured, easily searchable landscapes. Work on metaheuristic performances on various search landscapes may prove useful in this regard $[75,76]$.

\section{Acknowledgments}

This work has been conducted under the GIONET project funded by the European Commission, Marie Curie Programme, Initial Training Networks, Grant Agreement No. PIT-GA-2010-264509. The programming libraries OpenCV, TerraLib, SwarmOps, Qt and LibSVM were used in this work.

\section{Author Contributions}

Christoff Fourie conceived the concepts presented, implemented the methodologies, conducted the experimentation and wrote the manuscript. Elisabeth Schoepfer contributed to revision and editing.

\section{Conflicts of Interest}


The authors declare no conflict of interest.

\section{References}

1. Blaschke, T.; Hay, G.J.; Kelly, M.; Lang, S.; Hofmann, P.; Addink, E.; Feitosa, R.Q.; van der Meer, F.; van der Werff, H.; van Coillie, F.; et al. Geographic object-based image analysis-Towards a new paradigm. ISPRS J. Photogramm. Remote Sens. 2014, 87, 180-191.

2. Blaschke, T. Object based image analysis for remote sensing. ISPRS J. Photogramm. Remote Sens. 2010, 65, 2-16.

3. Hay, G.J.; Castilla, G. Geographic Object-Based Image Analysis (GEOBIA): A new name for a new discipline. In Object-Based Image Analysis: Spatial Concepts for Knowledge-Driven Remote Sensing Applications; Blaschke, T., Lang, S., Hay, G.J., Eds.; Springer: Berlin, Germany, 2008; pp. 75-90.

4. Addink, E.A.; van Coillie, F.M.B.; de Jong, S.M. Introduction to the GEOBIA 2010 special issue: From pixels to geographic objects in remote sensing image analysis. Int. J. Appl. Earth Obs. Geoinf. 2012, 15, 1-6.

5. Blaschke, T.; Burnett, C.; Pekkarinen, A. Image segmentation methods for object-based analysis and classification. In Remote Sensing Image Analysis: Including the Spatial Domain; de Jong, S.M., van Der Meer, F.D., Eds.; Kluwer Academic Publishers: Dordrecht, The Netherlands, 2004; pp. 211-236.

6. Hay, G.J.; Castilla, G.; Wulder, M.A.; Ruiz, J.R. An automated object-based approach for the multiscale image segmentation of forest scenes. Int. J. Appl. Earth Obs. Geoinf. 2005, 7, 339-359.

7. Soille, P. Constrained connectivity for hierarchical image partitioning and simplification. IEEE Trans. Pattern Anal. Mach. Intell. 2008, 30, 1132-1145.

8. Castilla, G.; Hay, G.J. Image objects and geographic objects. In Object-Based Image Analysis: Spatial Concepts for Knowledge-Driven Remote Sensing Applications; Blaschke, T., Lang, S., Hay, G.J., Eds.; Springer: Berlin, Germany, 2008; pp. 91-110.

9. Lang, S. Object-based image analysis for remote sensing applications: Modeling reality_Dealing with complexity. In Object-Based Image Analysis: Spatial Concepts for Knowledge-Driven Remote Sensing Applications; Blaschke, T., Lang, S., Hay, G.J., Eds.; Springer: Berlin, Germany, 2008; pp. 3-28.

10. Blaschke, T.; Strobl, J. What's wrong with pixels? Some recent developments interfacing remote sensing and gis. GeoBIT/GIS 2001, 6, 12-17.

11. Atkinson, P.M.; Aplin, P. Spatial variation in land cover and choice of spatial resolution for remote sensing. Int. J. Remote Sens. 2004, 25, 3687-3702.

12. Baatz, M.; Schäpe, A. Multiresolution segmentation: An optimization approach for high quality multiscale image segmentation. In Angewandte Geographische Informationsverarbeitung; Strobl, J., Blaschke, T., Griesebner, G., Eds.; Wichmann Verlag: Karlsruhe, Germany, 2000; Volume 12, pp. 12-23.

13. Castilla, G.; Hay, G.J.; Ruiz-Gallardo, J.R. Size-constrained region merging (SCRM): An automated delineation tool for assisted photointerpretation. Photogramm. Eng. Remote Sens. 2008, 74, 409-419. 
14. Baatz, M.; Hoffman, C.; Willhauck, G. Progressing from object-based to object-oriented image analysis. In Object-Based Image Analysis: Spatial Concepts for Knowledge-Driven Remote Sensing Applications; Blaschke, T., Lang, S., Hay, G.J., Eds.; Springer: Berlin, Germany, 2008; pp. 29-42.

15. Clewley, D.; Bunting, P.; Shepherd, J.; Gillingham, S.; Flood, N.; Dymond, J.; Lucas, R.; Armston, J.; Moghaddam, M. A python-based open source system for geographic object-based image analysis (GEOBIA) utilizing raster attribute tables. Remote Sens. 2014, 6, 6111-6135.

16. Doulaverakis, C.; Tzotsos, A.; Tsampoulatidis, I.; Gerontidis, N.; Argyridis, A.; Karantzalos, K.; Kompatsiaris, I.; Argialas, D. Gnorasi: A modular knowledge-based platform for object-based image analysis. South-Eastern Eur. J. Earth Obs. Geomat. 2014, 3, 473-476.

17. Passo, D.; Bias, E.; Brites, R.; Costa, G. Comparison of the accuracy of classifications generated by interimage and by interimage integrated with data mining. South-Eastern Eur. J. Earth Obs. Geomat. 2014, 3, 93-97.

18. Ouzounis, G.K.; Soille, P. The Alpha-Tree Algorithm; JRC Scientific and Policy Report; European Commission, Joint Research Centre: Ispra, Italy, 2012.

19. Drăguţ, L.; Tiede, D.; Levick, S.R. Esp: A tool to estimate scale parameter for multiresolution image segmentation of remotely sensed data. Int. J. Geogr. Inf. Sci. 2010, 24, 859-871.

20. Trias-Sanz, R.; Stamon, G.; Louchet, J. Using colour, texture, and hierarchical segmentation for high-resolution remote sensing. ISPRS J. Photogramm. Remote Sens. 2008, 63, 156-168.

21. Kersten, J.; Gähler, M.; Voigt, S. A general framework for fast and interactive classification of optical VHR satellite imagery using hierarchical and planar markov random fields. Photogramm. Fernerkund. Geoinf. 2010, doi:10.1127/1432-8364/2010/0066.

22. Frohlich, B.; Bach, E.; Walde, I.; Hese, S.; Schmullius, C.; Denzler, J. Land cover classification of satellite images using contextual information. In Proceedings of the ISPRS Workshop on 3D Virtual City Modelling, Regina, SK, Canada, 28 May 2013.

23. Tarabalka, Y.; Benediktsson, J.A.; Chanussot, J. Spectral-spatial classification of hyperspectral imagery based on partitional clustering techniques. IEEE Trans. Geosci. Remote Sens. 2009, 47, 2973-2987.

24. Mylonas, S.K.; Stavrakoudis, D.G.; Theocharis, J.B. Genesis: A ga-based fuzzy segmentation algorithm for remote sensing images. Knowl.-Based Syst. 2013, 54, 86-102.

25. Gould, S.; Gao, T.; Koller, D. Region-based segmentation and object detection. In Proceedings of the Advances in Neural Information Processing Systems, Vancouver, BC, Canada, 7-10 December 2009.

26. Bhanu, B.; Lee, S.; Ming, J. Adaptive image segmentation using a genetic algorithm. IEEE Trans. Syst. Man Cybern. 1995, 25, 1543-1567.

27. Feitosa, R.Q.; Costa, G.A.O.P.; Cazes, T.B.; Feijo, B. A genetic approach for the automatic adaptation of segmentation parameters. In Proceedings of the Geographic Object-Based Image Analysis (GEOBIA 2006), Salzburg, Austria, 4-5 July 2006.

28. Derivaux, S.; Forestier, G.; Wemmert, C.; Lefèvre, S. Supervised image segmentation using watershed transform, fuzzy classification and evolutionary computation. Pattern Recognit. Lett. 2010, 31, 2364-2374.

29. Fourie, C.; Schoepfer, E. Data transformation functions for expanded search spaces in geographic sample supervised segment generation. Remote Sens. 2014, 5, 3791-3821. 
30. Fourie, C.; Schoepfer, E. Classifier directed data transformations in sample supervised segment generation. In Proceedings of the Geographic Object-Based Image Analysis (GEOBIA 2014), Thessaloniki, Greece, 21-24 May 2014.

31. Bhanu, B.; Peng, J. Adaptive integrated image segmentation and object recognition. IEEE Trans. Syst. Man Cybern. 2000, 30, 427-441.

32. Pignalberi, G.; Cucchiara, R.; Cinque, L.; Levialdi, S. Tuning range image segmentation by genetic algorithm. EURASIP J. Appl. Signal Process. 2003, doi:10.1155/S1110865703303087.

33. Louis, S.J. Genetic Algorithms as a Computational Tool for Design; Indiana University: Bloomington, IN, USA, 1993.

34. Pal, S.K.; Bhandari, D.; Kundu, M.K. Genetic algorithms for optimal image enhancement. Pattern Recognit. Lett. 1994, 15, 261-271.

35. Yoda, I.; Yamamoto, K.; Yamada, H. Automatic acquisition of hierarchical mathematical morphology procedures by genetic algorithms. Image Vis. Comput. 1999, 17, 749-760.

36. Feitosa, R.Q.; Ferreira, R.S.; Almeida, C.M.; Camargo, F.F.; Costa, G.A.O.P. Similarity metrics for genetic adaptation of segmentation parameters. In Proceedings of the Geographic Object-Based Image Analysis (GEOBIA 2010), Ghent, Belgium, 29 June-2 July 2010.

37. Kowaliw, T.; Banzhaf, W.; Kharma, N.; Harding, S. Evolving novel image features using genetic programming-based image transforms. In Proceedings of the IEEE Congress on Evolutionary Computation (CEC'09), Trondheim, Norway, 18-21 May 2009.

38. Van Coillie, F.; Verbeke, L.P.; de Wulf, R.R. Feature selection by genetic algorithms in object-based classification of ikonos imagery for forest mapping in flanders, belgium. Remote Sens. Environ. 2007, 110, 476-487.

39. Fourie, C.; Schoepfer, E. Sample supervised search-centric approaches in Geographic Object-Based Image Analysis (GEOBIA): Concepts, state-of-the-art and a future outlook. In Earth Observation for Land and Emergency Monitoring-Innovative Concepts for Environmental Monitoring from Space; Balzter, H., Ed.; Wiley: Hoboken, NJ, USA, 2014 (In preperation).

40. Osman, J.; Inglada, J.; Christophe, E. Interactive object segmentation in high resolution satellite images. In Proceedings of the IEEE International Geoscience and Remote Sensing Symposium, Cape Town, South Africa, 12-17 July 2009.

41. Zhang, Y.J. A survey on evaluation methods for image segmentation. Pattern Recognit. 1996, 29, 1335-1346.

42. Achanccaray, P.; Ayma, V.; Jimenez, L.; Garcia, S.; Happ, P.; Feitosa, R.; Plaza, A. A free software tool for automatic tuning of segmentation parameters. South-Eastern Eur. J. Earth Obs. Geomat. 2014, 3, 707-712.

43. Melo, L.M.; Costa, G.A.O.P.; Feitosa, R.Q.; da Cruz, A.V. Quantum-inspired evolutionary algorithm and differential evolution used in the adaptation of segmentation parameters. In Proceedings of the Geographic Object-Based Image Analysis (GEOBIA 2008), Calgary, AL, Canada, 5-8 August 2008.

44. Happ, P.; Feitosa, R.Q.; Street, A. Assessment of optimization methods for automatic tuning of segmentation parameters. In Proceedings of the Geographic Object-based Image Analysis (GEOBIA 2012), Rio De Janeiro, Brazil, 7-9 May 2012. 
45. Fourie, C.; Schoepfer, E. Connectivity thresholds and data transformations in sample supervised segment generation. In Proceedings of the IEEE International Geoscience and Remote Sensing Symposium (IGARSS 2012), Melbourne, VIC, Australia, 21-26 July 2012.

46. Ferreira, R.S.; Feitosa, R.Q.; Costa, G.A.O.P. A multiscalar, multicriteria approach for image segmentation. In Proceedings of the Geographic Object-Based Image Analysis (GEOBIA 2012), Rio de Janeiro, Brazil, 7-9 May 2012.

47. Herold, H.; Meinel, G. Adaptive segmentation of heterogeneous images using metaheuristic evolutionary optimization. South-Eastern Eur. J. Earth Obs. Geomat. 2014, 3, 461-464.

48. Pedergnana, M.; Marpu, P.R.; Dalla Mura, M.; Benediktsson, J.A.; Bruzzone, L. A novel technique for optimal feature selection in attribute profiles based on genetic algorithms. IEEE Trans. Geosci. Remote Sens. 2013, 51, 3514-3528.

49. Wang, J.; Tan, Y. A novel genetic programming algorithm for designing morphological image analysis method. In Advances in Swarm Intelligence; Tan, Y., Shi, Y., Chai, Y., Wang, G., Eds.; Springer: Berlin, Germany, 2011.

50. Cagnoni, S. Evolutionary computer vision: A taxonomic tutorial. In Proceedings of the Hybrid Intelligent Systems (HIS 2008), Barcelona, Spain, 10-12 September 2008.

51. Fourie, C. A One Class Object Based System for Sparse Geographic Feature Identification; Stellenbosch University: Stellenbosch, South Africa, 2011.

52. Weicker, K.; Weicker, N. On the improvement of coevolutionary optimizers by learning variable interdependencies. In Proceedings of the Evolutionary Computation (CEC 1999), Washington, DC, USA, 6-9 July 1999.

53. Hoiem, D.; Efros, A.A.; Hebert, M. Closing the loop in scene interpretation. In Proceedings of the IEEE Conference on Computer Vision and Pattern Recognition (CVPR 2008), Anchorage, AK, USA, 24-26 June 2008.

54. Borenstein, E.; Ullman, S. Class-specific, top-down segmentation. In Computer Vision-Eccv 2002; Heyden, A., Sparr, G., Nielsen, M., Johansen, P., Eds.; Springer: Berlin, Germany, 2002; pp. 109-122.

55. Leibe, B.; Schiele, B. Interleaving object categorization and segmentation. In Cognitive Vision Systems: Sampling the Spectrum of Approaches; Christensen, H.I., Nagel, H., Eds.; Springer: Berlin, Germany, 2006; pp. 145-161.

56. Schölkopf, B.; Platt, J.C.; Shawe-Taylor, J.; Smola, A.J.; Williamson, R.C. Estimating the support of a high-dimensional distribution. Neural Comput. 2001, 13, 1443-1471.

57. Tax, D.M.; Duin, R.P. Support vector domain description. Pattern Recognit. Lett. 1999, 20, 1191-1199.

58. Khan, S.S.; Madden, M.G. A survey of recent trends in one class classification. In Artificial Intelligence and Cognitive Science; Springer: Berlin, Germany, 2010; pp. 188-197.

59. Vapnik, V.N.; Vapnik, V. Statistical Learning Theory; Wiley: New York, NY, USA, 1998; Volume 2.

60. Sun, L.; Yoshida, S.; Cheng, X.; Liang, Y. A cooperative particle swarm optimizer with statistical variable interdependence learning. Inf. Sci. 2012, 186, 20-39.

61. Achanta, R.; Shaji, A.; Smith, K.; Lucchi, A.; Fua, P.; Susstrunk, S. Slic superpixels compared to state-of-the-art superpixel methods. IEEE Trans. Pattern Anal. Mach. Intell. 2012, 34, 2274-2282. 
62. Vu, N.B.S. Image Segmentation with Semantic Priors: A Graph Cut Approach. Ph.D. Thesis, University of California, Santa Barbara, CA, USA, 2008.

63. Freddrich, C.M.B.; Feitosa, R.Q. Automatic adaptation of segmentation parameters applied to non-homogeneous object detection. In Proceedings of the Geographic Object-based Image Analysis (GEOBIA 2008), Calgary, AL, Canada, 5-8 August 2008.

64. Polak, M.; Zhang, H.; Pi, M. An evaluation metric for image segmentation of multiple objects. Image Vis. Comput. 2009, 27, 1223-1227.

65. Storn, R.; Price, K. Differential evolution-A simple and efficient heuristic for global optimization over continuous spaces. J. Glob. Optim. 1997, 11, 341-359.

66. Bartz-Beielstein, T. Experimental Research in Evolutionary Computation; Springer: Berlin, Germany, 2006.

67. Kennedy, J. Particle swarm optimization. In Encyclopedia of Machine Learning; Springer: Berlin, Germany, 2010; pp. 760-766.

68. Pedersen, M. Swarmops: Black-Box Optimization in ANSI C; Hvass Lab.: Southampton, UK, 2008.

69. Talbi, E. Metaheuristics: From Design to Implementation; Wiley: Hoboken, NJ, USA, 2009.

70. Demšar, J. Statistical comparisons of classifiers over multiple data sets. J. Mach. Learn. Res. 2006, 7, 1-30.

71. Wolpert, D.H.; Macready, W.G. No free lunch theorems for optimization. IEEE Trans. Evolut. Comput. 1997, 1, 67-82.

72. Wolpert, D.H. The lack of a priori distinctions between learning algorithms. Neural Comput. 1996, 8, 1341-1390.

73. Chang, C.-C.; Lin, C.-J. Libsvm: A library for support vector machines. ACM Trans. Intell. Syst. Technol. TIST 2011, doi:10.1145/1961189.1961199.

74. Vesterstrom, J.; Thomsen, R. A comparative study of differential evolution, particle swarm optimization, and evolutionary algorithms on numerical benchmark problems. In Proceedings of the IEEE Congress on Evolutionary Computation (CEC2004), Portland, OR, USA, 19-23 June 2004.

75. Watson, J. An introduction to fitness landscape analysis and cost models for local search. In Handbook of Metaheuristics; Gendreau, M., Potvin, J., Eds.; Springer US: New York, NY, USA, 2010.

76. Vanneschi, L.; Mussi, L.; Cagnoni, S. Hot topics in evolutionary computation. Intell. Artif. 2011, $5,5-17$.

(C) 2014 by the authors; licensee MDPI, Basel, Switzerland. This article is an open access article distributed under the terms and conditions of the Creative Commons Attribution license (http://creativecommons.org/licenses/by/4.0/). 


\section{CHAPTER 6: CONCLUSIONS}

A Methodological Avenue within Geographic Sample Supervised Segment Generation Additional Remarks on the Method Variants

Suggestions for Future Research 


\subsection{A Methodological Avenue within Geographic Sample Supervised Segment Generation}

This work considered the challenge of creating thematically accurate image segments in a remote sensing context. One methodological philosophy casts the generation of segments as a multidimensional search problem, referred to as sample supervised segment generation. This approach assists in generating more accurate image segments as well as increasing the autonomy of image analysis processes. The generic variant of the approach is severely limited in its ability to generate quality segments.

In this work, a general extension to geographic sample supervised segment generation was proposed. Adding additional fitness landscape constituents to such a general method was suggested. These constituents may encompass various data modification processes, image processing processes and decision-based operators functioning on segments. Such constituents may be interdependent with a given segmentation algorithm. Such method variants consisting of expanded fitness landscapes may generate superior segment results while requiring the same amount of user interaction. A computational overhead penalty may be involved, ranging from insignificant to impracticable, depending on the method variant details.

Three method variants, based on the concept of expanded fitness landscapes, were proposed, implemented and evaluated to demonstrate the general utility of such expanded method designs. The first proposed variant considered the addition of a range of computationally inexpensive data mapping functions as additional constituents. On a range of problem instances and under various conditions (including generalizability - cross-validation), most proposed variants performed better than the generic method variant, vastly so in select instances. Even though the mapping functions were very basic, the general results were encouraging, suggesting further research to refine such methods.

Secondly, a method variant was proposed that incorporates an attribute decision criterion constituent. A rich set of attributes may finely tailor segments, implemented here as thresholdable attributes in the context of connected component graph-based segmentation. Data mapping functions were also introduced alongside the attributes and core segmentation algorithm parameters. The method as a whole was analysed and the specific constituents were also scrutinised. Interestingly, in many cases attributes were found to be detrimental under cross-validated conditions - suggesting an overfitting problem. This result suggests that adding any constituents and expecting an immediate improvement is not feasible.

Finally another novel method variant was proposed that collected reference segment spectral data. The collected data were utilised for classification, generating a probability image. The generated probability image was used in various ways to map the data to a new domain. Sample collection, classifier tuning and a data hybridisation strategy forms the basic "tuneable" components/parameters in such a design. A few method variants were proposed based on this principle, with very promising results. A computational impact is observed, owing to the classification components, suggesting refinement with faster classifiers.

These three method variants and accompanying papers individually present various contributions and details. Collectively, they substantiate the argument for expanded fitness landscapes within geographic sample supervised segment generation. In two papers, a Friedman rank test, with a Nemenyi post-hoc test, was performed [1,2], which evaluates multiple methods under multiple 
conditions/datasets supporting this argument. Although, generally speaking, these methods show promise, they are proof-of-concept and not refined to deliver maximum achievable segment quality. Many other method designs may be pursued where data mapping functions or classification processes may be defined, improving on results in terms of quality, generalizability and process efficiency.

The improved performance in segment quality may suggest a greater uptake of these methods within GEOBIA workflows and systems. In its most simple form, such methods may simply be used as tools to select parameters within traditional rule-set development efforts. More elaborately, they may be used in semi-automated mapping systems, for example, in the context of rapid or emergency response mapping (with classification included). A user may be required simply to select a selection of elements of interest such as damaged buildings, certain vehicle types, flooded areas or refugee tents. Users should not have to concern themselves with minute method construction efforts that may be labour intensive, time consuming and subject to error. Nonetheless, details should be available for scrutiny and modification.

\subsection{Additional Remarks on the Method Variants}

A few additional comments/suggestions on the method variants are given.

\subsubsection{Data Mapping Function Variants}

The four mapping functions explored in this work are basic [3]. Finding a single good mapping function would be fruitful. Such a function should not increase fitness landscape dimensionality substantially (less than 20 parameters) and should be adaptable to a range of elements that would typically be of interest in an image. The selection of a promising mapping function may also be done via a statistical ranking (and statistical significance testing) under various problem conditions, as done with the other two papers [1,2]. Note that the characteristics of the accompanying segmentation algorithm should be considered intently when designing a mapping function.

Although the nature of mapping functions may be extremely diverse, the following aspects could be very useful:

- Multi-band synergies or information (such as in the transformation matrix)

- Pixel neighbourhood properties (detection of gradients/transition areas)

- Searchable/constructible map algebra

\subsubsection{Attribute Thresholding Variants}

A major problem with the constrained connectivity segmentation algorithm is the presence of single pixel segments/connected components in gradient zones. Within [2] this was not properly addressed. Incorporating a tuneable filter within sample supervised segment generation method variants in this context may be fruitful. Defining a dissimilarity measure as part of the search problem could be interesting. 


\subsubsection{Classifier Based Data Mapping Variants}

The method variants presented in [1] contain basic mechanisms for selecting pixel samples or for generating the probability imagery. A constituent may be defined where pixel samples are collected more elaborately - for the class of interest or for the other class (thus for one- or two-class classification). Spectral groupings may be defined as a searchable parameter and the tuning of classifier parameters may even be removed from the search space (e.g. SVM). A fitness landscape in this context may then be depicted as:

[Sample membership][Classifier parameters][Integration][Segment algorithm parameters]

\subsection{Suggestions for Future Research}

The general sample supervised segment generation approach may be improved in various ways, not necessarily related to expanded fitness landscapes. Suggestions for future research are presented to improve upon this general method. These suggestions include identifying method shortcomings or uncertainties encountered during this work, and initial thoughts on how they may be addressed. Potential avenues for continued research related to expanded fitness landscapes are also highlighted.

\subsubsection{Metric Fitness Landscape Characteristics}

A given metric should not merely be able to define accurately the best quality segments. The nature or topology of the fitness landscape should be taken into account. A metric should have a very good correlation, throughout its value range, with quality. Discontinuities or sporadic/erratic fitness landscapes may cause difficulty for the given search method, even for population-based derivative-free variants. During the course of this work, one of the used metrics, the Reference Bounded Segments Booster (RBSB) [4], displayed erratic behaviour in a few select instances where results did not agree with common sense or loosely with the other metrics. This is simply due to its more compact formulation (compared with the others, e.g. [3,5]), not having a rich description for various over- and under-segmentation scenarios.

Secondly, regions within the fitness landscape may be considered instead of specific points. The lowest (or highest) value in the fitness landscape may be situated near a steep rise (or decline). This may have an implication for generalizability. Under slightly different data or method conditions, such a scenario may lead to the generation of bad results. This scenario and potential solutions have been researched within the machine learning community for classifier free parameter tuning, e.g. [6]. Various processes could be considered to ensure better generalizability. A simple approach could be to analyse the neighbourhood of the best performing parameter set and make slight adjustments, if needed.

\subsubsection{Metrics Sensitive to Post-processes}

Metrics may also be defined more elaborately as opposed to simply observing area overlap and over/under-segmentation. Various aspects may be encoded into a metric, either weighted or as a multi- 
objective optimisation problem. Examples include edge-offsets, segment spectral content and neighbourhood characteristics.

A metric could also consider the potential difficulty of post-processing that would be needed to arrive at a final result. For example, common over-segmentation may not necessarily be extremely detrimental if some simple algorithm may merge segments afterwards. Segments may possibly be merged based on their geometry or spectral content. An algorithm could analyse segment properties and derive a score based on similarities or dissimilarities of said segments. Well-defined postprocessing algorithms may be related to these scores, suggesting a user may easily fix the problem (e.g. rule-sets). Over-segmentation may thus be awarded a smaller penalty than under-segmentation. This may be useful in instances where the target elements of interest have substantially different sizes, causing problems for a scale-space constraint or size constraint segmentation algorithm such as Multiresolution Segmentation (MS). Note that such processes may add a substantial additional computational impact to fitness evaluation.

\subsubsection{Unsupervised Metrics}

This research focused on supervised metrics or empirical discrepancy methods. Alternatively, empirical goodness methods may also be considered [7,8] in sample supervised segment generation (untested in remote sensing, to my knowledge). Predefined knowledge on image spectral properties or geometry could be used to define what would be considered good segments. A potential major advantage is the unsupervised nature of such an approach. A major disadvantage is that the system will not be aware if it is truly correct - it has no knowledge of the characteristics of elements of interest in a new image. Divergence from simple spectral and thematic correlations (common in VHR optical data) could be problematic. Nonetheless, it would be interesting and profitable to investigate in the context of remote sensing. Current unsupervised methods within remote sensing consider spectral content variance throughout a multi-scale image segmentation approach $[9,10]$.

\subsubsection{The influence of Sampling Size}

Within this work and similar works, the effect of sample size on sample supervised segment generation has not been investigated. Primarily the insight that could be gained from such an investigation is the minimum number of reference segments that would be needed to ensure a certain level of generalizability of the method. Digitising reference segments is labour intensive and should be minimised. Preliminary tests were conducted on sample sizes ranging from 5 to 50 . With fewer than 7 to 10 samples, method variants involving data mapping did not generalise well (two-fold crossvalidation). Methodology from classification may be borrowed for such an investigation, for example the authors of [11] investigate the changing classifier accuracies for SVMs with differentiating sampling sizes (remote sensing - crop classification - SVMs require considerably fewer training samples than classical parametric classifiers).

\subsubsection{Incorporate Easy Segment Delineation Tools}

Another extension that could be made to such a general method is the use of easy feature delineation tools, e.g. [12,13] instead of digitising. This would greatly ease the use of such methods. 
Sample supervised segment generation methods, mainly using area-offset metrics, may be sensitive to boundary offsets from manual or automated reference segment extraction. In addition, elements of interest may not have boundaries having strong gradients. These considerations suggest that easy segment delineation tools could be beneficial, but that the impact of quality reference segment delineation should enjoy further investigation.

\subsubsection{Tailored Search Methods}

In this work, a selection of standard metaheuristics was employed [14-16], empirically tuned. An initial attempt at metaheuristic meta-optimisation was made (successful - for a conference paper [17]), but the computational costs could not be accommodated for all work. For simpler fitness landscapes, the exact metaheuristic or its meta-optimisation might not be a major consideration $[3,18]$, but for more complex expanded fitness landscape variants it might need careful examination. The no-freelunch theorem is also valid in the context of search/optimisation [19]. A possible approach to increase the quality of results and reduce required computing costs in the context of these methods, might be to consider the creation of a set of benchmark methods and problems and test a range of metaheuristics. Alternatively, variants of metaheuristics with self-adapting metaparameters or hyperheuristics may be considered.

\subsubsection{Correlation Between Metrics and Classifier Accuracy}

A very interesting consideration is the relation, or correlation, between segment quality and final classification accuracies. Does optimal segment quality, in terms of elaborate balances between overand under-segmentation, relate to optimal classification results? It is conjectured that under conditions of rich attributes (various histograms, fast texture measures [20], etc.) and a well-constructed and tuned one-class SVM [21,22] (or other modern non-linear novelty detector) over-segmentation might be considered a lesser problem than under-segmentation. This could suggest that the simple conjectured correlation in this work and elsewhere does not generally hold. If such a sample supervised segment generation method is embedded in a larger method, also conducting classification, metrics should be compensated for this possibility.

\subsubsection{A Comparison with Finer Grained Image Analysis Methods}

Early on in this work it was mentioned that sample supervised segment generation is considered a tuning process and that construction processes conducting image analysis exist, e.g. [23-27]. It would be fruitful to compare such approaches in the context of quality segment generation in a remote sensing context (possible via classical mathematical morphology, cellular automata or genetic programming). Major aspects of interest would be the quality of final results, sampling size requirements, how methods generalise to unseen data, and very importantly, the required computing times.

\subsubsection{Classification Accuracy as Fitness Function}

Finally, the suggestion is made to consider fitness evaluation at the classification level and not necessarily at the segment generation level. If a specific target element is of interest, a novelty detector 
may be employed for classification (e.g. one-class SVM [21]). A combined sample supervised segment generation and novelty detection system has been demonstrated in [28], although not as a single fitness landscape. Various novelty detector quality measures may be considered for the fitness function that defines the fitness landscape, including the F-score or geometric means [22].

Segment quality may be removed or may form part of a weighted/multi-objective evaluation system. In the context of expanded fitness landscapes, attribute selection, attribute generation and classification algorithm parameter tuning may be added as constituents. Some aspects are unclear about such a potential design. Hopefully though, this suggestion provides some food for thought.

\subsection{References}

1. Fourie, C.; Schoepfer, E. Classifier directed data hybridization for geographic sample supervised segment generation. Remote Sensing. 2014, 6, 11852-11882.

2. Fourie, C. On attribute thresholding and data mapping functions in a supervised connected component segmentation framework. Remote Sensing. 2015, 7, 7350-7377.

3. Fourie, C.; Schoepfer, E. Data transformation functions for expanded search spaces in geographic sample supervised segment generation. Remote Sensing. 2014, 5, 3791-3821.

4. Freddrich, C.M.B.; Feitosa, R.Q. Automatic adaptation of segmentation parameters applied to non-homogeneous object detection. In ISPRS Archives Vol.No. XXXVIII-4/C1, Proceedings of Geographic Object-based Image Analysis (GEOBIA 2008), Calgary, Canada, 29 June - 2 July, 2008, Hay, G.J.; Blaschke, T.; Marceau, D., Eds; ISPRS.

5. Polak, M.; Zhang, H.; Pi, M. An evaluation metric for image segmentation of multiple objects. Image and Vision Computing. 2009, 27, 1223-1227.

6. Boardman, M.; Trappenberg, T. A heuristic for free parameter optimization with support vector machines. In Proceedings of Neural Networks, 2006. IJCNN'06. International Joint Conference on, IEEE.

7. Zhang, Y.J. A survey on evaluation methods for image segmentation. Pattern Recognition. 1996, 29, 1335-1346.

8. Chabrier, S.; Emile, B.; Rosenberger, C.; Laurent, H. Unsupervised performance evaluation of image segmentation. EURASIP Journal on Applied Signal Processing. 2006, Volume 2006, 1-12.

9. Drăguţ, L.; Tiede, D.; Levick, S.R. Esp: A tool to estimate scale parameter for multiresolution image segmentation of remotely sensed data. International Journal of Geographical Information Science. 2010, 24, 859-871.

10. Hay, G.J.; Castilla, G.; Wulder, M.A.; Ruiz, J.R. An automated object-based approach for the multiscale image segmentation of forest scenes. International Journal of Applied Earth Observation and Geoinformation. 2005, 7, 339-359.

11. Foody, G.M.; Mathur, A. A relative evaluation of multiclass image classification by support vector machines. IEEE Transactions on Geoscience and Remote Sensing. 2004, 42, 1335-1343.

12. Osman, J.; Inglada, J.; Christophe, E. Interactive object segmentation in high resolution satellite images, Paper presented at IEEE International Geoscience and Remote Sensing Symposium, Cape Town, 12-17 July 2009. 
13. Falcão, A.X.; Udupa, J.K.; Samarasekera, S.; Sharma, S.; Hirsch, B.E.; Lotufo, R.d.A. Usersteered image segmentation paradigms: Live wire and live lane. Graphical models and image processing. 1998, 60, 233-260.

14. Storn, R.; Price, K. Differential evolution-a simple and efficient heuristic for global optimization over continuous spaces. Journal of global optimization. 1997, 11, 341-359.

15. Kennedy, J. Particle swarm optimization. In Encyclopedia of machine learning, Springer: 2010; pp 760-766.

16. Pedersen, M. Swarmops: Black-box optimization in ansi c; Hvass Lab., Southampton, UK, 2008.

17. Fourie, C.; Schoepfer, E. Combining the heuristic and spectral domains in semi-automated segment generation, Paper presented at Geographic Object-based Image Analysis (GEOBIA 2012), Brazil, 7-9 May 2012.

18. Happ, P.; Feitosa, R.Q.; Street, A. Assessment of optimization methods for automatic tuning of segmentation parameters, Paper presented at Geographic Object-based Image Analysis (GEOBIA 2012), Rio De Janeiro, Brazil, 7-9 May 2012.

19. Wolpert, D.H.; Macready, W.G. No free lunch theorems for optimization. Evolutionary Computation, IEEE Transactions on. 1997, 1, 67-82.

20. Song, C.; Yang, F.; Li, P. Rotation invariant texture measured by local binary pattern for remote sensing image classification. In Proceedings of Education Technology and Computer Science (ETCS), 2010 Second International Workshop on, IEEE.

21. Schölkopf, B.; Platt, J.C.; Shawe-Taylor, J.; Smola, A.J.; Williamson, R.C. Estimating the support of a high-dimensional distribution. Neural computation. 2001, 13, 1443-1471.

22. Fourie, C.; Van Niekerk, A.; Mucina, L. Optimising a one-class svm for geographic object-based novelty detection, Paper presented at AfricaGeo, Cape Town, South Africa, 31 May - 2 June 2011.

23. Kowaliw, T.; Banzhaf, W.; Kharma, N.; Harding, S. Evolving novel image features using genetic programming-based image transforms. In Proceedings of Evolutionary Computation (CEC'09), IEEE Congress on, Trondheim, Norway, 18-21 May, IEEE.

24. Harding, S.; Leitner, J.; Schmidhuber, J. Cartesian genetic programming for image processing. In Genetic programming theory and practice x, Riolo, R.; Vladislavleva, E.; Ritchie, M.D.; Moore, J.H., Eds. Springer: New York, United States of America, 2013; pp 31-44.

25. Yoda, I.; Yamamoto, K.; Yamada, H. Automatic acquisition of hierarchical mathematical morphology procedures by genetic algorithms. Image and Vision Computing. 1999, 17, 749-760.

26. Rosin, P.L. Image processing using 3-state cellular automata. Computer Vision and Image Understanding. 2010, 114, 790-802.

27. Frohlich, B.; Bach, E.; Walde, I.; Hese, S.; Schmullius, C.; Denzler, J. Land cover classification of satellite images using contextual information. In Proceedings of The ISPRS Workshop on 3D Virtual City Modelling, Regina, Canada, 28 May 2013, ISPRS.

28. Fourie, C. A one class object based system for sparse geographic feature identification. Stellenbosch University, Stellenbosch, 2011. 


\section{APPENDIX A - CONFERENCE PAPERS}

\section{GEOBIA 2012}

Peer reviewed paper - Talk

Fourie, C.; Schoepfer, E. Combining the heuristic and spectral domains in semi-automated segment generation

Rio De Janeiro, Brazil

7-9 May 2012

\section{IGARSS 2013}

Peer reviewed paper - Talk

Fourie, C.; Schoepfer, E. Connectivity thresholds and data transformations for sample supervised segment generation

Melbourne, Australia

21-26 July 2013

\section{GEOBIA 2014}

Extended Illustrated Abstract - Talk

Fourie, C.; Schoepfer, E. Classifier directed data transformations in sample supervised segment generation

Thessaloniki, Greece

21-24 May 2014 


\title{
COMBINING THE HEURISTIC AND SPECTRAL DOMAINS IN SEMI-AUTOMATED SEGMENT GENERATION
}

\author{
C. E. Fourie*, E. Schoepfer \\ German Aerospace Center (DLR), German Remote Sensing Data Center (DFD), 82234 \\ Oberpfaffenhofen, Germany - (christoffel.fourie, elisabeth.schoepfer)@dlr.de
}

KEY WORDS: Meta-Heuristic, Semi-Automation, Segmentation, Spectral Transformation, Rapid Mapping

\begin{abstract}
:
The generation of thematically accurate image segments or delineating land-cover elements is a common objective and challenge in Geographic Object Based Image Analysis (GEOBIA). A core notion to the applicability of segmentation algorithms for partitioning these land-cover elements is that said elements typically have some spectral or other homogeneity criteria that allow successful segmentation, to varying degrees. One approach that addresses this challenge models the parameterised segmentation process as a search problem. The search method is provided with a reference of optimal desired output. This idea is extended in this work by suggesting the encoding of spectral space transformations as additional variables to such a search problem. The automatic exploration and transformation of the spectral domain can allow for a closer correlation between thematic and spectral similarity of the land-cover elements of interest, thus aiding the segmentation process. Two simple spectral transformation methods functioning in conjunction with two scale-space constraint image segmentation algorithms are presented to illustrate this concept. A statistically significant improvement in segmentation results can be obtained consistently in acceptable time with this approach with off-the-shelf meta-heuristics for our test areas. It is also shown with the algorithms used in this study that the segmentation algorithm parameters (heuristic domain) are dependent on the spectral transformation parameters (spectral domain) to achieve optimal results. This necessitates simultaneous optimisation of these two domains.
\end{abstract}

\section{INTRODUCTION}

Image segmentation is a ubiquitous paradigm in scientific disciplines that are concerned with information extraction from imagery. In the discipline of remote sensing, thematically accurate segments, or geographic objects, are typically the desired end result of a segmentation process. The semi-automation of generating these geographic-objects (single or multi-scale) holds value in domains concerned with monitoring or emergency response mapping, where user interaction is required to be non-exhaustive and turnover times short. In such a context the emphasis falls on user assisted information extraction rather than full autonomy of the information extraction process or using extensive pre-developed solutions. 1

\footnotetext{
${ }^{1}$ Corresponding author
} 
One school of thought moderates the process of semi-automated object or geographic-object generation to an optimisation problem (Bhanu et al., 1995; Pignalberi et al., 2003; Feitosa et al., 2006; Fredrich \& Feitosa, 2008; Derivaux et al., 2010). The search space of all possible results, or segment sets, is traversed to find a solution that most closely matches a small reference set provided by a user. Empirical discrepancy metrics (Zhang, 1996), especially area based accuracy metrics (Pignalberi et al. 2003; Fredrich \& Feitosa, 2008; Feitosa et al., 2010), are commonly employed to judge the resemblance of any segment set and the reference set. It is assumed that if the given segmentation algorithm and parameter(s) are adequate for segmenting the reference; it will be adequate for previously unseen examples.

The search space of such an approach usually consists of the real or discrete valued parameters of the segmentation algorithm employed. Due to the potentially complex and large search spaces, coupled with the computationally expensive nature of image segmentation, stochastic population based search methods are preferred. No guarantees can be made that the optimal solution can be found or if a quality solution even exists for the given scenario. Results remain dependent on the inherent suitability of the segmentation algorithm to the problem. To our knowledge, thus far only the segmentation algorithm parameters, or the Heuristic Domain (HD), are considered variables to such a method.

Another line of research considers the effects different colour or spectral space representations of the input data have on the performances of image processing tasks. Examples include measuring the effects different colour space representations have on image segmentation (Busin, Vandenbroucke \& Macaire, 2008; Kwok, Ha \& Fang, 2009), automatic iterative colour space selection for a given segmentation problem (Busin et al., 2004) and the application and derivation of illumination invariant colour spaces (Chong, Gortler \& Zickler, 2008; Shan, Yan \& Wang, 2007).

The concept of modelling example driven segmentation as a search problem is extended by suggesting the addition of simple measures of Spectral Domain (SD) transformation within the search function. Inspired by the abovementioned work on colour space transformations and concepts of lowmid-high level image processing cue integration (Kumar, Torr \& Zisserman, 2010), it is suggested that low-level (image transformation) and mid-level (image segmentation) image processing steps are combined and optimised simultaneously. Typically, any analysis, refinement or classification will be done on original untransformed spectral data, although bounded by segment borders derived with the help of additional image processing steps.

Closer correlations between spectral and thematic similarity can be found if the data is allowed to be transformed, assisting the segmentation algorithm on the user defined problem. It is also briefly shown here that these two domains are interdependent and needs to be optimised simultaneously in our experiments.

It is not uncommon for users to use expert knowledge of the problem at hand in defining optimal input to a segmentation problem, for example segmenting with a vegetation index band (EVI, NDVI) if concerned with a vegetation application. It is suggested that the user can be unburdened with this task if examples of desired output are available.

In section 2 of this paper the method for simultaneously optimising the spectral and heuristic domains is presented, along with some details on the search algorithms, segmentation algorithms, fitness functions and spectral transformation functions implemented. Section 3 describes the study area and test data used. Quantitative comparisons of the proposed extension to the original method are 
presented in Section 4. Conclusions on the characteristics of the method and suggestions for future work are presented in Section 5.

\section{OPTIMISING ALGORITHM AND SPECTRAL TRANSFORMATION PARAMETERS}

\section{Combined heuristic and spectral domain search}

The proposed method to simultaneously optimise the heuristic domain (segmentation algorithm parameters) and the spectral domain (transformed image input used in segmentation) is illustrated in Figure 1 (hereafter called HD + SD search). HD + SD search consists of a core optimisation layer giving and receiving input from a fitness score generation layer, which in turn receives input from a simple image input layer.

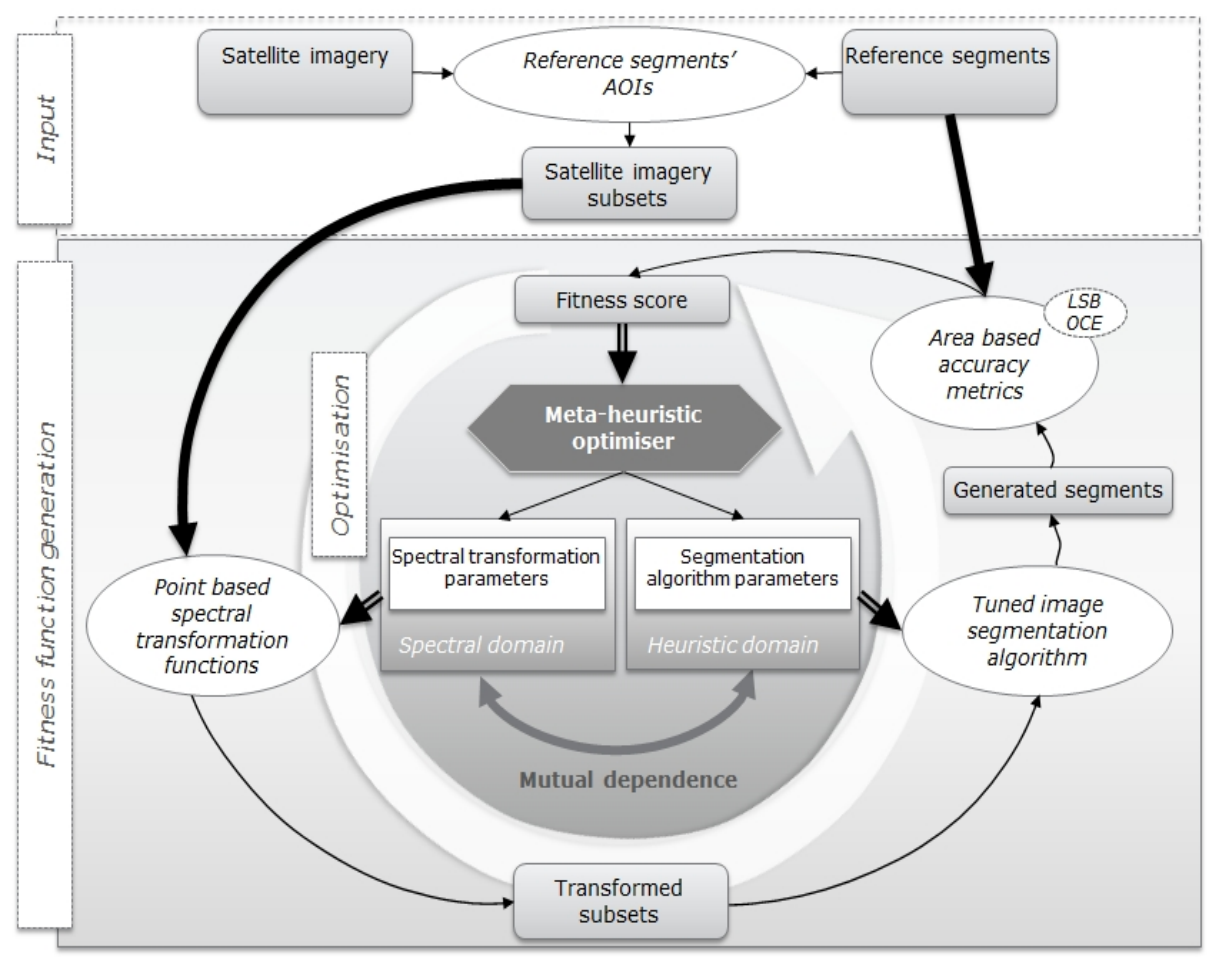

Figure 1. Method for simultaneously optimising the spectral and heuristic domains in semi-supervised segment generation (SD + HD search).

As input, the method accepts a multi-band image and a Boolean raster of the same dimensions, delineating reference segments of desired outputs. Small subsets of the satellite image, centred on the coordinates (areas of interest) of the user defined reference segments, are extracted and used for all subsequent processing. Subsetting saves computing time by avoiding repeated segmentation of unnecessary areas.

The core of the method consists of an iterative optimisation/search algorithm taking as input a single value, called the fitness score. Optimisers employed are presented in section 2.2. As output the optimisation algorithm produces multidimensional real and/or discrete valued sets depicting spectral transformation parameters (SD) and segmentation algorithm parameters (HD). The dimensionality of the overall problem depends on the used segmentation algorithm and spectral transformation method. 
During iterations of the optimisation algorithm the fitness score generation layer is invoked and provided with SD and HD parameter sets provided by the optimisation layer. The SD parameters are used as input to a transformation function that changes the spectral space representation of the imagery subsets, resulting in new transformed subsets. Two simple transformation functions are employed and described in section 2.3. These transformed subsets are used as input to a segmentation algorithm tuned with the HD parameters provided by the optimisation layer. Two segmentation algorithms are tested with this approach and are described in section 2.4. The resulting generated segments are evaluated against the reference segments (taken from the input layer) with the aid of area based empirical discrepancy metrics (described in section 2.5.), producing the fitness score. Subsequently the fitness score is returned to the optimisation layer, invoking a new iteration of the optimisation algorithm.

The SD + HD method terminates after a certain number of iterations of the search algorithm have been performed. The output of the SD + HD search is an interdependent spectral transformation parameter set and segmentation algorithm parameter set that was found most suited to the problem (as judged by the fitness function).

\section{Optimisers}

Two common stochastic population based meta-heuristic optimisers were implemented and tested in this study, namely the Differential Evolution (DE) (Storn \& Prince, 1995) algorithm and the Particle Swarm Optimisation (PSO) (Kennedy \& Eberhart, 1995) algorithm. Multi-objective meta-optimisation (Pederson \& Chipperfield, 2009) was performed on both algorithms to tune their controlling parameters. Figure 2 illustrates typical fitness curves observed with these two algorithms for our example problems. For all our experiments it was found that between 600 and 1000 iterations were sufficient to achieve near optimal solution fitness. The DE strategy provided slightly better final fitness scores compared to a standard PSO strategy and was subsequently used for all further experimentation.

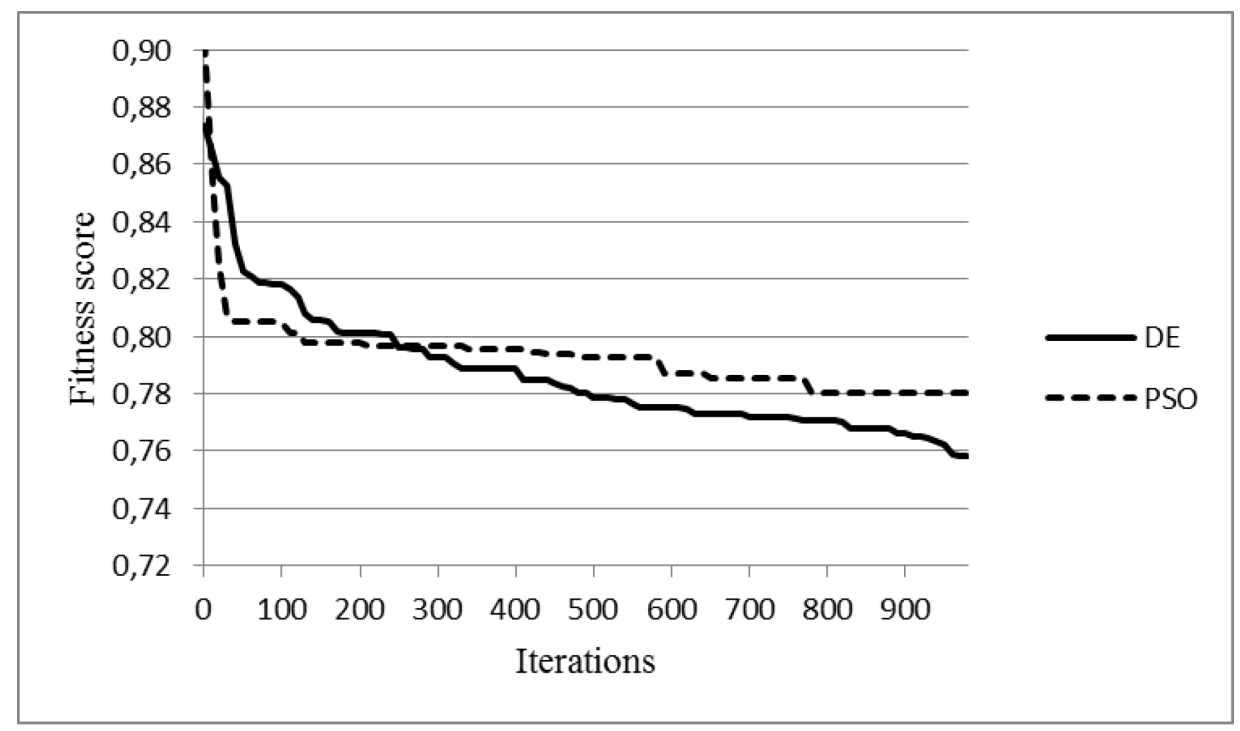

Figure 2. A typical example of fitness traces generated with the meta-optimised DE and PSO algorithms on HD + SD search problems. 


\section{Spectral transformation functions}

Two transformation methods are implemented, namely a transformation matrix and a simple histogram modification function. All used images have three spectral bands. The transformation matrix converts the original three band spectral space $(b 1, b 2, b 3)$ to a new space $(n 1, n 2, n 3)$ with the following equation:

$$
\left[\begin{array}{l}
n 1 \\
n 2 \\
n 3
\end{array}\right]=\left[\begin{array}{lll}
a & b & c \\
d & e & f \\
g & h & i
\end{array}\right] \times\left[\begin{array}{l}
b 1 \\
b 2 \\
b 3
\end{array}\right]
$$

Where variables a through i define the transformation matrix and have numerical bounds of $[-0.2$, 1] in our tests.

The histogram modification function, called spectral split, consists of four variables. Three variables define positions (p1, p2, p3) within the histograms of the three separate bands (range of [0, 255]), while the fourth (h) defines the magnitude with which pixels around these points in the histogram are modified. If a pixel value in band $x$ satisfies any of the following two conditional statements it is changed accordingly:

If valuex $<\mathrm{px}$ and valuex $>\mathrm{px}-\mathrm{h}$ then valuex $=$ valuex $-(\mathrm{h}-\mathrm{px}-$ valuex $)$.

If valuex $>$ px and valuex $<\mathrm{px}+\mathrm{h}$ then valuex $=$ valuex $+(\mathrm{h}-$ valuex $-\mathrm{px})$.

These two point based image transformation functions only modify spectral values based on their own values and either some variables (in the case of spectral split) or variables and spectral values of other bands (in the case of the transformation matrix). Using spectral split adds an additional four variables to the optimisation problem, while using the transformation matrix adds an additional nine.

\section{Segmentation algorithms}

The HD + SD search method is tested with two segmentation algorithms, namely the Simple Linear Iterative Clustering (SLIC) (Radhakrishna et al., 2010) algorithm and a region merging segmentation algorithm variant, called Multiresolution Segmentation (MS) (Baatz \& Schäpe, 2000). As the name suggests SLIC is an iterative clustering segmentation algorithm (K-means) combining the spectral and spatial properties/dimensions of an image into a single Euclidian space. Regions or segments are clustered in an unsupervised manner in this combined spectral/spatial space. The SLIC algorithm holds two parameters, one called "Scale" controlling relative segment size and "M"; controlling segment compactness. SLIC was developed for generating superpixels to be used in a parts-based information extraction paradigm. It is used with some liberty here in a thematic segmentation context due to algorithm elegancy/simplicity and speed efficiency.

The MS region merging algorithm has three main parameters entitled „Scale“, „Colour" and „Compactness". Additional parameters control the contributions (weights) that the different image input bands have in influencing segment merging. Interestingly, this simple form of band weighting constitutes a low-level image processing or modification task; encoded within the actual segmentation algorithm. For simplicity bands are not weighted in this work. 
Concerns could be raised regarding the practical feasibility (computing time) of modelling semisupervised segmentation as a search problem. It can be noted that in our experiments with the SD + HD search method using SLIC as the segmentation algorithm the search process typically finished after 1-2 minutes while the MS algorithm took 4-5 minutes (standard desktop computer with no algorithm multithreading or code optimisations, using very high resolution (VHR) imagery and numerous reference segments).

\section{Empirical discrepancy metrics}

Two area-based empirical discrepancy metrics are implemented and tested with the HD + SD search method, namely the Larger Segments Booster (LSB) (Fredrich \& Feitosa, 2008) and a modified version of the Object-level Consistency Error (OCE) (Polak et al., 2009). Both these metrics can compensate for over- and under-segmentation. Both use measures of false positives and false negatives to quantify the percentage of overlap; however they strongly differ in their implementations of handling over-segmentation.

The LSB metric compensates for over-segmentation by counting the number of pixels intersecting the reference segment and using this value as a penalisation factor. The OCE metric handles false positives and false negatives per individual segment that has some overlap with the reference segment. With OCE, over-segmentation is penalised via the summation of individual segment overlap results; weighted by the percentage cover of said overlap with the reference segment (see Polak et al., 2009 for a full formulation). Both metrics have a numerical range of $[0,1]$ with a value of 1 indicating no match and a value of 0 indicating a perfect match with the reference segments.

\section{TEST AREA AND DATA}

The viability and characteristics of the proposed method is demonstrated via the task of identifying habitable structures in Internally Displaced Persons (IDP) camps in East Africa. Relief agencies need accurate estimations of the number and sizes of habitable structures in these camps to model population size. GeoEye-1 and QuickBird imagery subsets (5 ha - 50 ha) of three IDP camps, in Kenya, Somalia and Ethiopia were selected, for simplicity referred to by their hosting countries. 2 Land-cover mapping and structure counting of these settlements are routinely performed to provide relief agencies with updated maps and information.

The three sites display different structure characteristics (see Figure 3). The Ethiopia site mainly consists of easily identifiable white tents or huts draped with white tent nylon. The land-cover elements of interest thus display strong within element spectral homogeneity and are also relatively homogeneous in the scale-space. One could practically use a single-scale segmentation approach for this site. The other two sites (Kenya and Somalia) display different structural and spectral characteristics and variation in structure size, constituting a more difficult problem. In practise a multiclass and multi-scale approach would be suggested for these sites. To demonstrate the SD + HD search

2 EO data provided by the ESA managed GSC-DA, funded under ESA - EC Agreement on the Implementation of the Space Component of Global Monitoring for Environment and Security (GMES). 
method, it is attempted to segment all structures using a single segmentation layer and thematic class. For each site between ten and twenty reference structures were digitised, to be used as reference segments input.

\section{RESULTS AND DISCUSSION}

\section{Heuristic domain search versus combined heuristic and spectral domain search}

The HD + SD search method is quantitatively compared with the variant of semi-supervised segmentation not performing any spectral space transformations. For each test site (Ethiopia, Kenya and Somalia) the search methodology is tested by performing no spectral space transformations, using spectral split and the transformation matrix; on both segmentation algorithms implemented. Experiments for each problem scenario were repeated for 25 runs to obtain a measure of the standard deviation of the results. Table 1 lists the mean metric fitness scores and standard deviations obtained using the OCE metric, while Table 2 lists the same using the LSB metric.

\begin{tabular}{|l|l|l|l|l|}
\hline OCE & & No transformation & Spectral split & Transformation matrix \\
\hline Ethiopia & SLIC & $0,47 \pm 0,00$ & $0,44 \pm 0,02$ & $0,37 \pm 0,01$ \\
& MS & $0,35 \pm 0,01$ & $0,32 \pm 0,02$ & $\mathbf{0 , 2 8} \pm \mathbf{0 , 0 3}$ \\
\hline Kenya & SLIC & $0,80 \pm 0,00$ & $0,78 \pm 0,01$ & $0,71 \pm 0,02$ \\
& MS & $0,77 \pm 0,01$ & $0,75 \pm 0,01$ & $\mathbf{0 , 6 9} \pm \mathbf{0 , 0 2}$ \\
\hline Somalia & SLIC & $0,80 \pm 0,01$ & $0,78 \pm 0,01$ & $0,73 \pm 0,02$ \\
& MS & $0,76 \pm 0,00$ & $0,76 \pm 0,01$ & $\mathbf{0 , 6 7} \pm \mathbf{0 , 0 2}$ \\
\hline
\end{tabular}

Table 1. OCE metric scores comparison of the HD + SD search approach versus a HD only approach.

\begin{tabular}{|l|l|l|l|l|}
\hline LSB & & No transformation & Spectral split & Transformation matrix \\
\hline Ethiopia & SLIC & $0,48 \pm 0,00$ & $0,47 \pm 0,01$ & $0,45 \pm 0,01$ \\
& MS & $0,44 \pm 0,01$ & $0,41 \pm 0,01$ & $\mathbf{0 , 4 1} \pm \mathbf{0 , 0 2}$ \\
\hline Kenya & SLIC & $0,83 \pm 0,00$ & $0,79 \pm 0,01$ & $\mathbf{0 , 6 8} \pm \mathbf{0 , 0 2}$ \\
& MS & $0,79 \pm 0,01$ & $0,77 \pm 0,02$ & $0,68 \pm 0,02$ \\
\hline Somalia & SLIC & $0,91 \pm 0,00$ & $0,87 \pm 0,01$ & $0,76 \pm 0,02$ \\
& MS & $0,84 \pm 0,02$ & $0,82 \pm 0,02$ & $\mathbf{0 , 7 0} \pm \mathbf{0 , 0 1}$ \\
\hline
\end{tabular}

Table 2. LSB metric scores comparison of the HD + SD search approach versus a HD only approach.

Tables 1 and 2 illustrate marked improvement in average metric scores when employing spectral domain transformation functions within the search method. In all examples the simple transformation matrix approach produced the best results. In our experiments using a meta-optimised DE search 
algorithm very small standard deviations in obtained results are observed; potentially an indication that optimal results for these scenarios are reached, bearing in mind segmentation algorithm and spectral transformation function capabilities. Such a methodology can also be used to compare different segmentation algorithms for a given application and in selecting a proper spectral transformation function.

The distributions of the HD versus HD + SD search results are all statistically significant with a $1 \%$ confidence interval (student's t-test). The results achieved with the best combination of segmentation algorithms and spectral transformation functions are typed in bold.

Figure 3 shows the best performing segmentations achieved (small subsets) using SLIC for all three sites using the HD only search strategy (3(a), 3(d), 3(g)) compared with that of a HD + SD search using spectral split (3(b), 3(e), 3(h)) and a transformation matrix (3(c), 3(f), 3(i)).
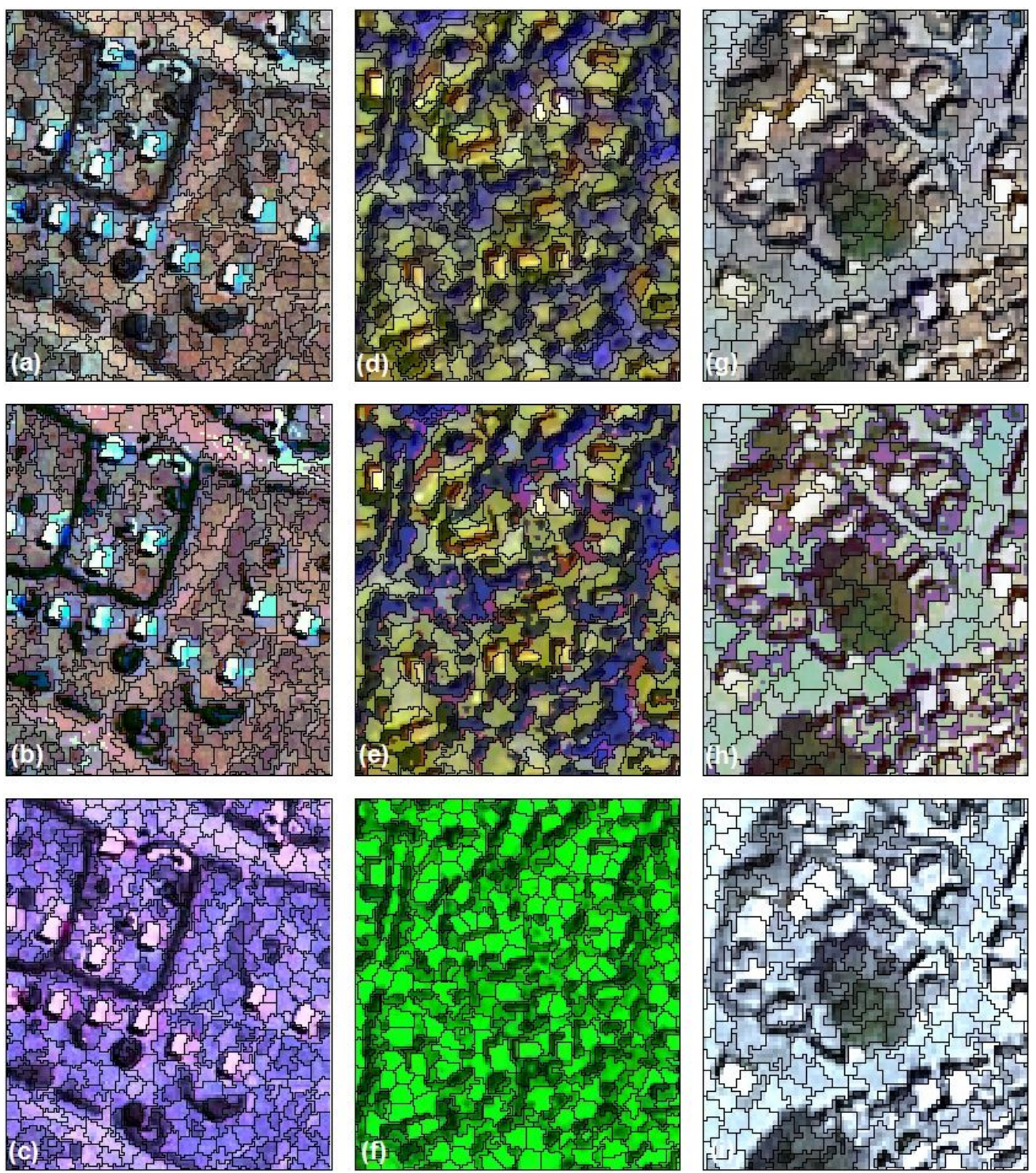

Figure 3. Best performing segmentation results for the Ethiopia (a-c), Kenya (d-f) and Somalia (g-i) test sites. 


\section{A luminance-chrominance space as base input}

The above experiment is repeated by using a luminance-chrominance colour space as the basic input to the HD and HD + SD search methods. One dimension of the CIELAB luminance-chrominance colour space (L) used here defines brightness while the other two dimensions (A and B) define the colour components. Using the CIELAB colour space as base representation is compared with the original red, green and blue (RGB) colour space to illustrate the potential of using more elaborately transformed colour spaces; although selected in a non-automated manner in this example. Table 3 lists the results for the Kenya test site using the OCE metric as fitness function.

\begin{tabular}{|l|l|l|l|l|}
\hline Kenya & & No transformation & Spectral split & Transformation matrix \\
\hline SLIC & RGB & $0,80 \pm 0,00$ & $0,78 \pm 0,01$ & $0,71 \pm 0,02$ \\
& CIELAB & $0,77 \pm 0,00$ & $0,76 \pm 0,01$ & $\mathbf{0 , 6 8} \pm \mathbf{0 , 0 2}$ \\
\hline MS & RGB & $0,77 \pm 0,01$ & $0,75 \pm 0,01$ & $0,69 \pm 0,02$ \\
& CIELAB & $0,74 \pm 0,01$ & $0,71 \pm 0,01$ & $\mathbf{0 , 6 2} \pm \mathbf{0 , 0 3}$ \\
\hline
\end{tabular}

Table 3. OCE metric scores comparing RGB and CLIELAB colour spaces as base input to the HD and HD + SD search methods.

For this specific problem the use of a luminance-chrominance colour space improved the results, irrespective of the spectral transformation method used. A substantial improvement in results is observed comparing the use of the original RGB colour space (No transformation) with the CIELAB space using the spectral transformation matrix.

Comparison of heuristic domain parameter values using different spectral domain transformation techniques

The dependence of the HD parameters on the used SD method and parameters is illustrated in Tables 4 and 5. Table 4 lists the average parameter values of the SLIC algorithm obtained (best results) for the three test sites while Table 5 lists the same for the MS algorithm.

\begin{tabular}{|l|l|l|l|l|}
\hline SLIC & & No transformation & Spectral split & Transformation matrix \\
\hline Ethiopia & Scale & $6,00 \pm 0,00$ & $6,24 \pm 0,59$ & $5,96 \pm 0,20$ \\
& $\mathrm{M}$ & $33,40 \pm 0,75$ & $31,04 \pm 9,24$ & $28,20 \pm 6,75$ \\
\hline Kenya & Scale & $7,16 \pm 0,54$ & $7,48 \pm 1,55$ & $7,40 \pm 2,10$ \\
& $\mathrm{M}$ & $32,64 \pm 5,47$ & $29,12 \pm 7,36$ & $33,92 \pm 5,15$ \\
\hline Somalia & Scale & $5,00 \pm 0,00$ & $5,48 \pm 0,75$ & $5,64 \pm 0,48$ \\
& $\mathrm{M}$ & $31,76 \pm 6,84$ & $29,60 \pm 7,87$ & $28,96 \pm 7,30$ \\
\hline
\end{tabular}

Table 4. Average SLIC parameters obtained using the HD and HD + SD search methods. 


\begin{tabular}{|l|l|l|l|l|}
\hline MS & & No transformation & Spectral split & Transformation matrix \\
\hline Ethiopia & Scale & $12,88 \pm 0,52$ & $16,68 \pm 7,49$ & $8,52 \pm 4,30$ \\
& Colour & $0,10 \pm 0,00$ & $0,27 \pm 0,28$ & $0,32 \pm 0,31$ \\
\hline & Compactness & $0,97 \pm 0,07$ & $0,75 \pm 0,29$ & $0,71 \pm 0,25$ \\
\hline Kenya & Scale & $14,72 \pm 2,20$ & $11,68 \pm 2,62$ & $7,32 \pm 1,64$ \\
& Colour & $0,11 \pm 0,01$ & $0,11 \pm 0,01$ & $0,15 \pm 0,11$ \\
& Compactness & $0,77 \pm 0,10$ & $0,83 \pm 0,19$ & $0,72 \pm 0,22$ \\
\hline Somalia & Scale & $8,48 \pm 0,70$ & $9,28 \pm 1,64$ & $6,04 \pm 1,71$ \\
& Colour & $0,10 \pm 0,01$ & $0,11 \pm 0,02$ & $0,19 \pm 0,17$ \\
& Compactness & $0,90 \pm 0,06$ & $0,88 \pm 0,13$ & $0,84 \pm 0,17$ \\
\hline
\end{tabular}

Table 5. Average MS parameters obtained using the HD and HD + SD search methods.

The standard deviation of the Scale parameter of the SLIC algorithm (Table 4) is low with a slight difference in mean values when comparing different SD transformation techniques. In contrast, the $\mathrm{M}$ parameter displayed large deviation in optimal results, suggesting the Scale parameter to be of greater importance to the problem. Figure 4 illustrates this notion by plotting fitness results obtained by segmenting a test area with all combinations of Scale and M parameters (no SD transformations).

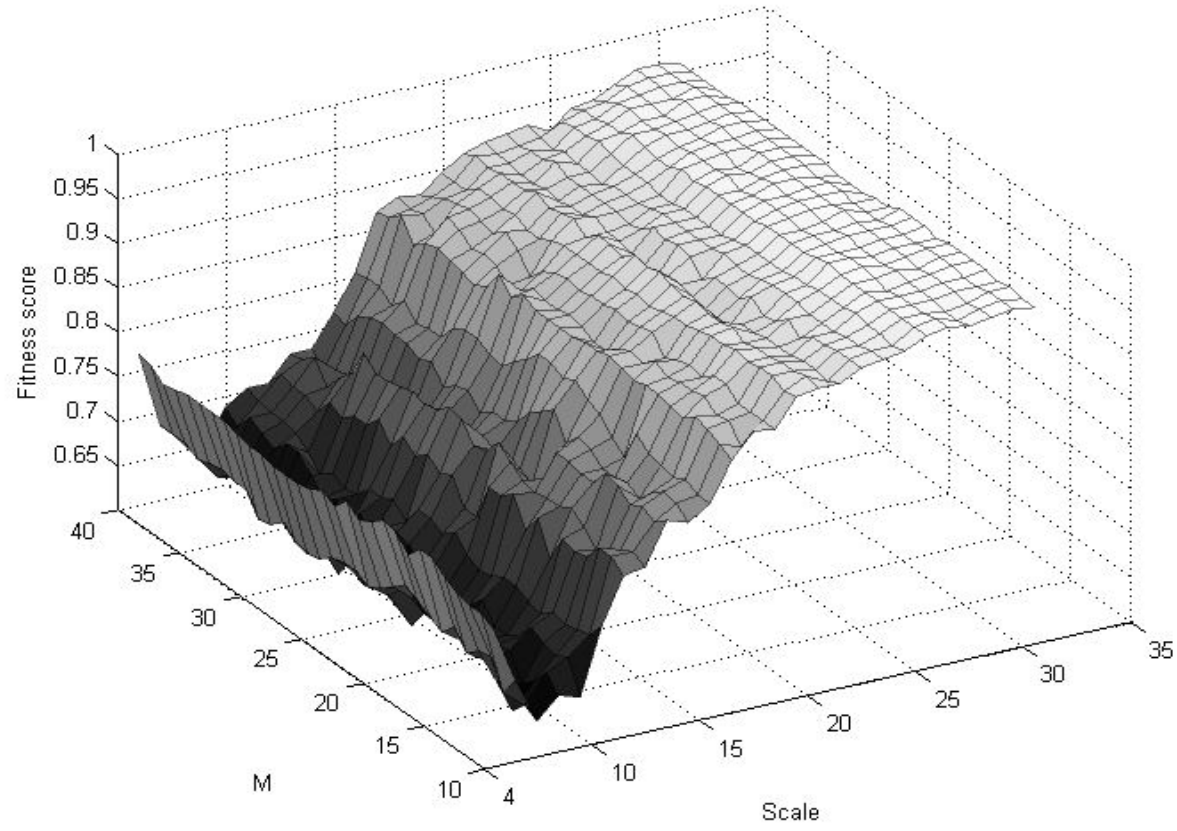

Figure 4. Fitness plot of the SLIC parameters for an arbitrary problem.

In contrast to the SLIC algorithm parameters, the MS parameters showed marked differences (Table 5) in optimal HD parameters obtained using different SD transformation techniques, suggesting stronger parameter interdependence for this algorithm. These results illustrate the influence of the SD parameters on the optimal HD parameters. The amount of influence that the SD parameters have on optimal HD parameters depends on the nature of the segmentation algorithm under consideration. 


\section{CONCLUSION}

In this study a general methodology that combines the search for effective spectral transformation function parameters and segmentation algorithm parameters in a single search problem was presented and tested. The method was compared with a simpler variant where no input data modification is performed, and was shown to improve results measured via area based empirical discrepancy metrics. It should be noted that the capabilities and performances of the employed meta-heuristics, segmentation algorithms, spectral transformation methods and fitness functions should be carefully considered in such an approach. Specific algorithms might perform poorly on certain problems.

Investigating this methodology with segmentation algorithms less constraint in the scale space is called for. Encoding more complex spectral transformation techniques, briefly demonstrated in section 4.2 by using a static luminance-chrominance colour space as base input, might prove useful. Transformation functions that modify spectral values based on neighbourhood properties, or so called neighbourhood operators, could also potentially aid in generating better segments. Efficiency in generalizability of such an approach will constitute future research, specifically investigating the performances of candidate low-level transformation techniques on commonly used segmentation algorithms and mapping problems.

\section{ACKNOWLEDGEMENTS}

This work has been conducted under the GIONET project funded by the European Commission, Marie Curie Programme, Initial Training Networks, Grant Agreement number PIT-GA-2010-264509 and in the framework of the GMES Emergency Response project SAFER with funding from the European Community`s Seventh Framework Programme.

\section{REFERENCES}

Baatz, M., Schäpe, A., 2000. Multiresolution Segmentation - an optimization approach for high quality multi-scale image segmentation. In: Angewandte Geographische Informationsverarbeitung XII, Strobl, J., Blaschke, T., Griesebner, G., (eds), Wichmann, Heidelberg, Germany, pp. 12-23.

Bhanu, B., Lee, S., Das, S., 1995. Adaptive image segmentation using genetic and hybrid search methods. IEEE Transactions on Aerospace and Electronic Systems, 31(4), pp. 1268-1291.

Busin, L., Vandenbroucke, N., Macaire, L., 2008. Color Spaces and Image Segmentation. Advances in Imaging and Electron Physics, 151(2), pp. 65-168.

Busin, L., Vandenbroucke, N., Macaire, L., Postaire, J. G., 2004. Color space selection for unsupervised color image segmentation by histogram multithresholding. In Proceedings of the IEEE International Conference on Computer Vision, Singapore, pp. 203-206.

Chong, H. Y., Gortler, S. J., Zickler, T., 2008. A perception-based color space for illuminationinvariant image processing. ACM Transactions on Graphics, 27(3), pp. 1-7.

Derivaux, S., Forestier, G., Wemmert, C., Lefèvre, S., 2010. Supervised image segmentation using watershed transform, fuzzy classification and evolutionary computation. Pattern Recognition Letters, 31(15), pp. 2364-2374. 
Feitosa, R. Q., Ferreira, R. S., Almeida, C. M., Camargo, F. F., Costa, G. A. O. P., 2010. Similarity metrics for genetic adaptation of segmentation parameters. Paper presented at the Geographic Object-Based Image Analysis (GEOBIA) 2010 conference held 29 June - 2 July 2010. Ghent, Belgium.

Feitosa, R. Q., Costa, G. A. O. P., Cazes, T. B., Feijo, B., 2006. A genetic approach for the automatic adaptation of segmentation parameters. Paper presented at the Geographic Object-Based Image Analysis (GEOBIA) 2006 conference held 4 - 5 July 2006. Salzburg, Austria.

Fredrich, C. M. B., Feitosa, R. Q., 2008. Automatic adaptation of segmentation parameters applied to inhomogeneous objects detection. Paper presented at the Geographic Object-Based Image Analysis (GEOBIA) 2008 conference held 6 - 7 August 2008. Calgary, Canada.

Kennedy, J., Eberhart, R. C., 1995. Particle swarm optimization. Paper presented at the IEEE international conference on neural networks held 26 - 28 June 1995. Cambridge, United Kingdom.

Kumar, M. P., Torr, P. H. S., Zisserman, A., 2010. OBJCUT: Efficient Segmentation Using Top-Down and Bottom-Up Cues. IEEE Transactions on Pattern Analysis and Machine Intelligence, 32(3), pp. 530-545.

Kwok, N., Ha, Q., Fang, G., 2009. Effect of color space on color image segmentation. In Proceedings of 2nd International Congress on Image and Signal Processing, Tianjin, China, pp. 1-5.

Pedersen, M. E. H., Chipperfield, A. J., 2010. Simplifying Particle Swarm Optimization. Applied Soft Computing, 10(2), pp. 618-628.

Pignalberi, G., Cucchiara, R., Cinque, L., Levialdi, S., 2003. Tuning range image segmentation by genetic algorithm. EURASIP Journal on Applied Signal Processing, 8, pp. 780-790.

Polak, M., Zhang, H., Pi, M., 2009. An evaluation metric for image segmentation of multiple objects. Image and Vision Computing. 27(8), pp. 1223-1227.

Radhakrishna, A., Shaji, A., Smith, K., Lucchi, A., Fua, P., Susstrunk, S., 2010. SLIC Superpixels. EPFL Technical Report 149300.

Shan, Y., Yang, F., Wang, R., 2007. Color space selection for moving shadow elimination. In Proceedings of the Fourth International Conference on Image and Graphics (ICIG), Chengdu, China, pp. 496-501.

Storn, R., Price, K., 1995. Differential evolution - a simple and efficient adaptive scheme for global optimization over continuous spaces. International Computer Science Institute technical report TR-95-012. Berkeley.

Zhang, Y. J., 1996. A survey on evaluation methods for image segmentation. Pattern Recognition, 29(8), pp. 1335-1346. 


\title{
CONNECTIVITY THRESHOLDS AND DATA TRANSFORMATIONS FOR SAMPLE SUPERVISED SEGMENT GENERATION
}

\author{
Christoff Fourie, Elisabeth Schoepfer \\ German Aerospace Center (DLR), German Remote Sensing Data Center (DFD), 82234 \\ Oberpfaffenhofen, Germany - (christoffel.fourie, elisabeth.schoepfer)@dlr.de
}

\begin{abstract}
Image analysis techniques based on mathematical morphology principles such as attribute filters and constrained connectivity shows promise for specific remote sensing applications; such as the identification or delineation of urban structures in VHR optical data. This could be attributed to the flexibility of these techniques to employ a range of morphological and spectral attributes, with controlling values, in segmenting imagery. In this work a sample supervised image analysis approach is investigated whereby the controlling values of attributes, and in the case of constrained connectivity, the controlling parameters, are modeled as a multidimensional search problem. The search landscape is defined via a spatial accuracy metric observing both over and under segmentation. This method is extended with the addition of data transformations, allowing for higher segmentation accuracies. Preliminary results are given comparing accuracies of this approach and a traditional segmentation method, comparing different data transformation functions and presenting some search method profiling.
\end{abstract}

Index Terms - Image Analysis, Mathematical Morphology, Optimization, Spatial Metrics

\section{Introduction}

Sample supervised image analysis entails the automatic construction or tuning of image processing algorithms for a problem given a subset or selection of the desired output $[1,2,3]$. The desired output could be a pre-classified, segmented or digitized area, commonly referred to as the reference. Accuracy measures, typically various metrics based on spatial and/or spectral properties [4], are used to measure the quality of any produced solution to the provided reference. In the context of this general approach finding suitable image processing to duplicate the reference can be modeled as a search problem. The search landscape consists of the multidimensional parameter space of the image processing algorithms employed $[1,3]$.

This general methodology was transferred to a remote sensing image analysis context [5] where the parameter space of a commonly employed segmentation algorithm is searched. Recent work also 
extended this concept by encoding additional data transformations [6] or homogeneity predicates [7] to the segmentation algorithm parameter space.

In this work this general approach is extended to and profiled in the domain of image segmentation approaches (single-scale) based on mathematical morphology concepts. Constrained Connectivity (CC) $[8,9]$ is a hierarchical image simplification/segmentation technique having alpha-connected components [8] as atomic units as opposed to image grey levels [10]. As with attribute filters, a wide range of morphological and spectral attributes can be encoded into this method as decision predicates, although at the expense of additional computing time. A multidimensional search landscape is modeled consisting of the two parameters of the $\mathrm{CC}$ algorithm in addition to five spectral and morphological attributes. A selection of data transformation functions is also encoded into the model, illustrating the usefulness of additional transformations to achieve increased segmentation accuracies.

\section{Constrained connectivity in sample supervised segment generation}

Figure 1 illustrates the sample supervised method for segment generation with the CC algorithm, additional attributes and an extra data transformation function. The method takes as input an image with a selection of reference segments provided by an image analyst.

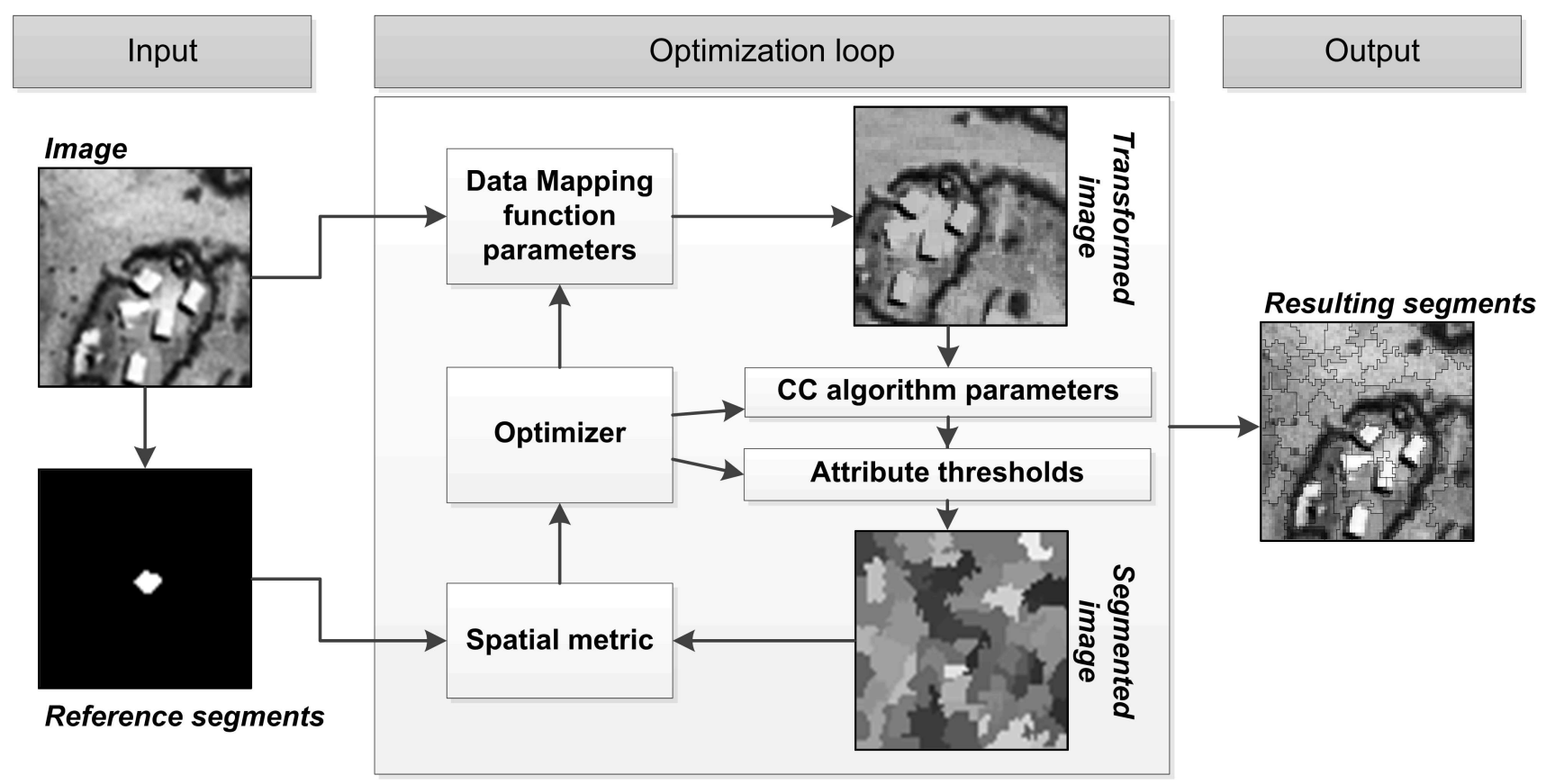

Figure 1. Sample supervised method for segment generation with the $\mathrm{CC}$ algorithm, additional attributes and a data transformation function.

A stochastic population based real-valued search method, in our case the Differential Evolution meta-heuristic (DE) [11], is used to search the combined CC algorithm parameter, attribute and data transformation space. Table 1 illustrates an example encoding of an eleven dimensional modeled search space along with the numerical bounds of the parameters (integer values converted to real). 
Table 1. An example modeled search space.

\begin{tabular}{|c|c|c|}
\hline Component & Parameter/Attribute & Value range \\
\hline Spectral split function [6] & Band 1 position & $0-255$ \\
\cline { 2 - 3 } & Band 2 position & $0-255$ \\
\cline { 2 - 3 } & Band 3 position & $0-255$ \\
\cline { 2 - 3 } & Height & $0-255$ \\
\hline \multirow{4}{*}{ CC parameters } & Alpha & $1-255$ \\
\cline { 2 - 3 } & Wglobal & $1-255$ \\
\hline Attributes & Area & $1-500$ \\
\cline { 2 - 3 } & Standard deviation & $0-50$ \\
\cline { 2 - 3 } & Perimeter & $4-1000$ \\
\cline { 2 - 3 } & Smoothness & $1-30$ \\
\cline { 2 - 3 } & Compactness & $1-30$ \\
\hline
\end{tabular}

At iterations of the search algorithm loop the image (subsets for efficiency) is first filtered with a region growing algorithm to remove outliers and then transformed according to a data transformation function, in this example the spectral split function [6]. This transformed image is segmented with the automatically tuned CC algorithm, with the automatically thresholded attributes as additional criteria. The Object-level Consistency Error (OCE) spatial metric [12] is used to evaluate the similarity of the generated segments with that of the reference. The OCE metric value range is [0 - 1] with zero indicating a perfect match. The metric observes both over and under segmentation. After a given number of search iterations the method terminates with the parameter set and resulting segments producing the best results given as output.

\section{PRELIMINARY RESULTS}

\subsection{Data and sampling criteria}

Figure 2 shows the three preprocessed very high spatial resolution optical imagery test sites used in this study, depicting various refugee camps in East Africa. The aim is to automatically correctly segment, with a single segment layer, the structures following the sample supervised image analysis approach described above. Practically, relief agencies would like to know the number of structures and also their sizes for population modeling purposes. For each site between ten and twenty representative structures are digitized and provided as reference segments to the method. To obtain a measure of generalizability of the performance of the method, segmentation results are averaged over all provided reference structures for a given image. The Ethiopia site (Figure 2(a)) constitutes a relatively easy problem with the features of interest being white nylon draped tents having very similar spatial and spectral characteristics. The Kenya (Figure 2(b)) and Somalia (Figure 2(c)) sites provide more spatially and spectrally heterogeneous elements of interest. 

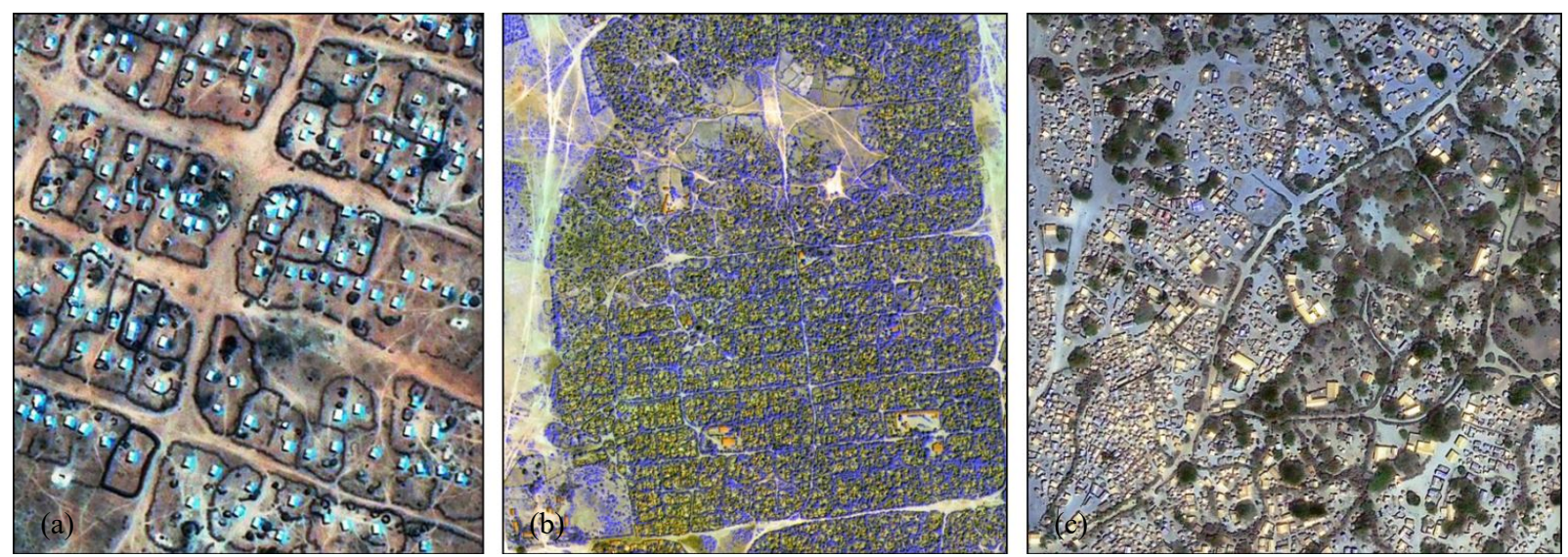

Figure 2. Three test sites depicting refugee camps in (a) Ethiopia, (b) Kenya and (c) Somalia.

\subsection{Segmentation accuracies}

A comparative experiment measuring segmentation accuracies was conducted with the sample supervised method, and search space as depicted in Table 1, functioning in four different ways: Only optimizing the two parameters of the $\mathrm{CC}$ algorithm (CC), optimizing the $\mathrm{CC}$ parameters and the attribute thresholds (CC + Attributes), optimizing the $\mathrm{CC}$ parameters and the transformation function parameters $(\mathrm{CC}+$ Transforms $)$ and finally allowing the method to optimize $\mathrm{CC}$ parameters, attributes and transformation parameters (CC + Attr + Trans). Table 2 lists the achieved OCE metric scores for each test site investigated. For a baseline comparison the same experiment is repeated using the Multiresolution Segmentation (MS) region merging algorithm [13].

Table 2. OCE metric scores for the three test sites using the CC algorithm with added attributes and a data transformation function.

\begin{tabular}{|l|l|l|l|l|l|}
\hline OCE metrics scores & $\mathrm{CC}$ & $\begin{array}{l}\mathrm{CC}+ \\
\text { Attributes }\end{array}$ & $\begin{array}{l}\mathrm{CC}+ \\
\text { Transforms }\end{array}$ & $\begin{array}{l}\mathrm{CC}+ \\
\text { Attr }+ \\
\text { Trans }\end{array}$ & $\begin{array}{l}\text { MS } \\
\text { algorithm }\end{array}$ \\
\hline Ethiopia & 0.291 & 0.258 & 0.262 & 0.250 & 0.350 \\
\hline Kenya & 0.774 & 0.748 & 0.762 & 0.698 & 0.770 \\
\hline Somalia & 0.841 & 0.791 & 0.836 & 0.743 & 0.760 \\
\hline
\end{tabular}

The most flexible variant, the CC algorithm with extra encoded attributes and a data transformation function produced the best results. In this test the addition of a selection of attributes proved more beneficial than adding the simple data transformation function. The CC + Attr + Trans variant of the method display competitive results with the popular MS algorithm. Figure 3 illustrates the behavior of the CC (OCE: 0.81), CC + Transforms (OCE: 0.73) and CC + Att + Trans (OCE: 0.71) variants of the method trained on a single structure of interest. The compactness attribute was needed to separate the bright bottom quadrant of the reference structure with the generally bright neighboring structure (Figure 3(d)). 


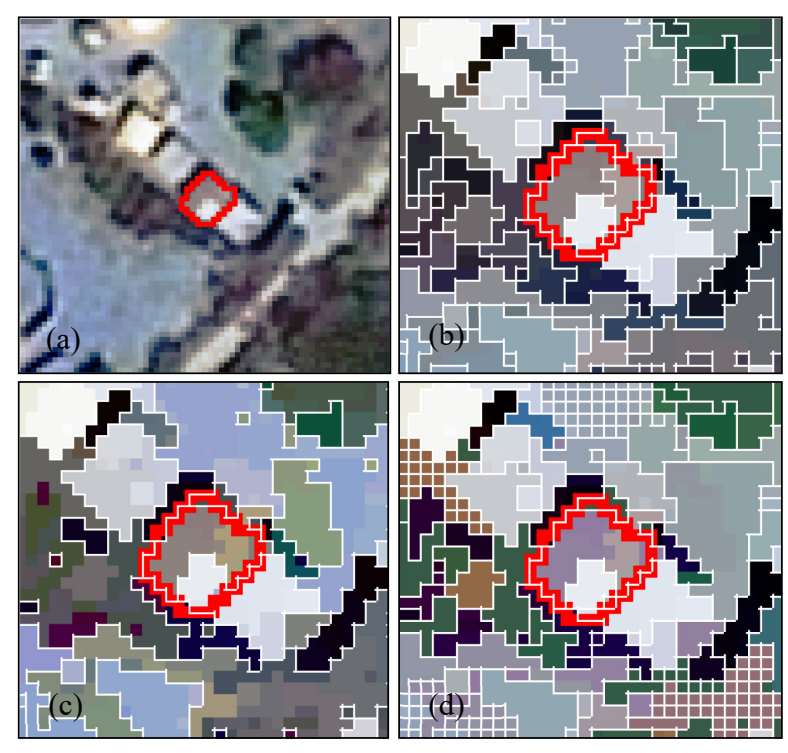

Figure 3. Segmentation results for a reference structure (red/in bold) in the Somalia site. The original image is shown (a), best results of the CC method (b), CC + Transforms method (c) and CC + Attr + Trans method (d).

\subsection{Data transformation function parameters as additional variables to the search landscape}

Table 3 illustrates different achieved OCE metric scores using a selection of image enhancement or data transformation functions. The genetic contrast enhancement [14] method modifies an image based on four parameters; which modify pixels based on both local and global properties. The ten parameters genetic transform [15] method employs four polynomial functions and accompanying weighting parameters to stretch and/or compress certain spectral ranges within the image. The linear transform method enhances the importance of certain bands or correlations in an image [6].

Table 3. Comparison of the performances of three different data transformation functions.

\begin{tabular}{|l|l|l|l|l|}
\hline $\begin{array}{l}\text { OCE metrics } \\
\text { scores }\end{array}$ & CC + Attr & $\begin{array}{l}\text { CC }+ \text { Attr }+ \\
\text { Genetic contrast }\end{array}$ & $\begin{array}{l}\text { CC }+ \text { Attr }+ \\
\text { Genetic transform }\end{array}$ & $\begin{array}{l}\text { CC }+ \text { Attr }+ \\
\text { Linear transform }\end{array}$ \\
\hline Ethiopia & 0.258 & 0.222 & 0.242 & 0.237 \\
\hline Kenya & 0.748 & 0.713 & 0.792 & 0.774 \\
\hline Somalia & 0.791 & 0.777 & 0.766 & 0.765 \\
\hline
\end{tabular}

These results illustrate the potential usefulness of added data mapping or transformation functions in such a general method. The mechanism whereby it functions is to allow data modification so that the given homogeneity predicates (spectral and/or morphological) of the segmentation method can perform better on the given problem; as defined by the multiple reference segments.

Employing two of the transformation functions on the Kenya test site listed in Table 3 resulted in a worse OCE score. This suggests that candidate data transformation methods should be carefully considered and evaluated in such an implementation as degradation in results might be possible. This could happen due to agents being trapped in local optima in a rugged search landscape or due to the general ineffectiveness of a transformation method for the given problem. 


\subsection{Search landscape characteristics and search problem difficulty}

Figure 4 illustrates the relation of the central Wglobal parameter of the CC + Attr + Trans method with one of the lesser important parameters of the spectral split function in terms of OCE metric scores. The accuracies displayed are the average over 20 reference structures. All other parameters were assigned random constant values. Typically as one increases the Wglobal parameter, larger segments will result. A narrow range (15-20) of Wglobal values will give good results, bordered by values that will either under or over segment the features of interest. The Band 2 position parameter has some minor influence on the results. The figure illustrates some minor multimodality of the search landscape (parameter interdependence) with only two parameters of eleven being altered.

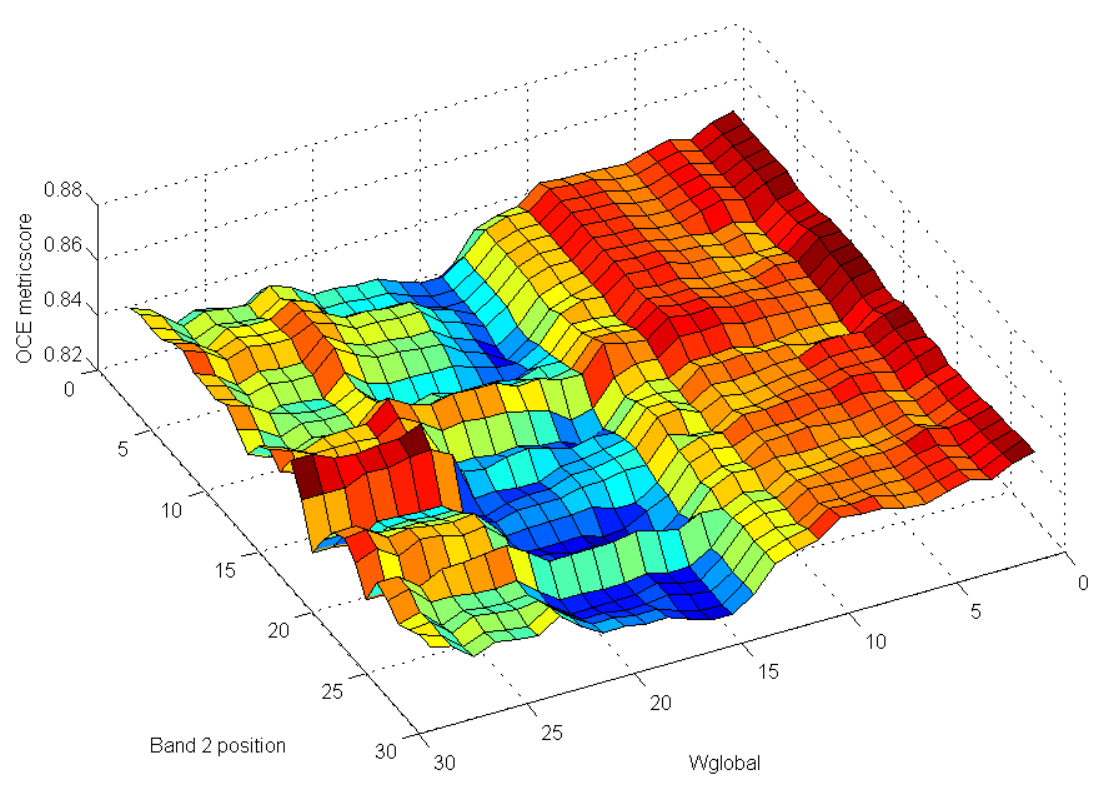

Figure 4. Two dimensions of the search landscape plotted in terms of OCE scores with the $\mathrm{CC}+\mathrm{Attr}+$ Trans variant of the method.

A main drawback of the general methodology described here is the large computational costs of the fitness evaluations. Rugged search surfaces, numerous local minima and the high dimensionality of the search landscape might cause problems for some search methods, such as those based on calculating derivatives. All previous experiments were conducted with the DE search method given 50 agents and 1300 fitness evaluations. Table four profiles three search methods in terms of maximum achieved OCE scores for the CC + Attr + Trans variant of the method (Table 1 search space, Somalia test site) given a fixed number of allowed fitness landscape evaluations.

Table 4. Maximum fitness scores achieved by different search methods given a fixed number of fitness evaluations for the Somalia test site. Results are averaged over five consecutive runs.

\begin{tabular}{|l|l|l|l|}
\hline OCE metric scores & Random search & $\begin{array}{l}\text { Simulated } \\
\text { annealing }\end{array}$ & $\begin{array}{l}\text { Differential } \\
\text { evolution }\end{array}$ \\
\hline
\end{tabular}




\begin{tabular}{|l|l|l|l|}
\hline 500 iterations & $0.786 \pm 0.004$ & $0.785 \pm 0.003$ & $0.784 \pm 0.002$ \\
\hline 1000 iterations & $0.779 \pm 0.004$ & $0.782 \pm 0.002$ & $0.781 \pm 0.002$ \\
\hline 1500 iterations & $0.779 \pm 0.004$ & $0.779 \pm 0.001$ & $0.777 \pm 0.004$ \\
\hline
\end{tabular}

The population based metaheuristic (DE) provided the best OCE scores under all iteration constraints, suggesting the single agent simulated annealing method is being trapped in local minima. This suggests careful consideration of the employed search method.

\section{Conclusions}

The modeling of CC with the ability to accommodate extra decision attributes, or potentially other mathematical morphology approaches such as attribute filters or attribute profiles, in a sample supervised image analysis context shows promise. Profiling of the processing time and achieved accuracies of the different variants of the method with other common segmentation algorithms is planned. A major concern is the computational costs of such approaches, as sample supervised image analysis generally advocates near real time interactive systems.

An investigation into the appropriateness of using specific attributes in terms of computing costs, under efficient implementations [10], and achieved accuracies might be insightful. Some attributes might be more beneficial for common remote sensing image analysis applications. It might be interesting to contrast the relative usefulness of parameter controlled data transforms with computationally expensive descriptive attributes (also considering search landscape dimensionality).

\section{Acknowledgements}

This work has been conducted under the GIONET project funded by the European Commission, Marie Curie Programme, Initial Training Networks, Grant Agreement number PIT-GA-2010-264509

\section{References}

[1] B. Bhanu, S. Lee and S. Das, Adaptive image segmentation using genetic and hybrid search methods. IEEE Transactions on Aerospace and Electronic Systems, vol. 31, no. 4, pp. 1268-1291, 1995.

[2] I. Yoda, K. Yamamoto, and H. Yamada, Automatic acquisition of hierarchical mathematical morphology procedures by genetic algorithms, Image and Vision Computing, vol. 17, nr. 10, pp. 749-760, 1999.

[3] S. Derivaux, G. Forestier, C. Wemmert and S. Lefèvre, Supervised image segmentation using watershed transform, fuzzy classification and evolutionary computation, Pattern Recognition Letters, vol. 31, nr. 15, pp. 2364-2374, 2010.

[4] J.Y. Zhang, A survey on evaluation methods for image segmentation, Pattern Recognition, vol. 29, nr. 8, pp. 1335-1346, 1996.

[5] R.Q. Feitosa, G.A.O.P. Costa, T.B. Cazes and B. Feijo, A genetic approach for the automatic adaptation of segmentation parameters, Paper presented at the Geographic Object-Based Image Analysis (GEOBIA) 2006 conference held 4 - 5 July 2006. Salzburg, Austria. 
[6] C.E. Fourie and E. Schoepfer, Combining the heuristic and spectral domains in semi-automated segment generation. Paper presented at the Geographic Object-Based Image Analysis (GEOBIA) 2012 conference held 7-9 May 2012, Rio de Janeiro, Brazil.

[7] R.S. Ferreira, R.Q. Feitosa and G.A.O.P. Costa, A multiscalar, multicriteria approach for image segmentation, Paper presented at the Geographic Object-Based Image Analysis (GEOBIA) 2012 conference held 7-9 May 2012, Rio de Janeiro, Brazil.

[8] P. Soille, Constrained connectivity for hierarchical image partitioning and simplification, IEEE Transactions on Pattern Analysis and Machine Intelligence, vol. 30, nr. 7, pp. 1132-1145, 2008.

[9] L. Najman, On the equivalence between hierarchical segmentations and ultrametric watersheds, Journal of Mathematical Imaging and Vision, vol. 40, nr. 3, pp. 231-247, 2011.

[10] M. Dalla Mura, J. A. Benediktsson, B. Waske, and L. Bruzzone, Morphological attribute filters for the analysis of very high resolution images, in Proc. IEEE IGARSS, Cape Town, South Africa, July 12-19, vol. 3, pp. 97-100, 2009.

[11] R. Storn and K. Price, Differential evolution - A simple and efficient adaptive scheme for global optimization over continuous spaces, International Computer Science Institute technical report TR-95-012. Berkeley, 1995.

[12] M. Polak, H. Zhang and M. Pi, An evaluation metric for image segmentation of multiple objects, Image and Vision Computing, vol. 27, nr. 8, pp. 1223-1227, 2009.

[13] M. Baatz and A. Schäpe, Multiresolution Segmentation - an optimization approach for high quality multi-scale image segmentation, In: Angewandte Geographische Informationsverarbeitung XII, J. Strobl, T. Blaschke, G. Griesebner, (eds), Wichmann, Heidelberg, Germany, pp. 12-23, 2000.

[14] C. Muntaenu and A. Rosa, Towards automatic image enhancement using genetic algorithms, Proceedings of the congress on evolutionary computation, vol. 2, pp. $1535-1542,2000$.

[15]M. S. Shyu and J. J. Leou, A genetic algorithm approach to color image enhancement, International Journal of Pattern Recognition, vol. 31, nr. 7, pp. 871-880, 1998.

(C) 2013 IEEE. Reprinted, with permission, from Fourie, C.; Schoepfer, E. Connectivity thresholds and data transformations for sample supervised segment generation, Paper presented at Geoscience and Remote Sensing Symposium (IGARSS 2013), IEEE International, Melbourne, 21-26 July. 


\title{
CLASSIFIER DIRECTED DATA TRANSFORMATIONS IN SAMPLE SUPERVISED SEGMENT GENERATION
}

\author{
Christoff Fourie $^{\mathrm{a}, *}$, Elisabeth Schoepfer ${ }^{\mathrm{a}}$ \\ ${ }^{a}$ Researcher, German Aerospace Center (DLR), German Remote Sensing Data Center (DFD), 82234 \\ Oberpfaffenhofen, Germany \\ *Corresponding author: christoffel.fourie@dlr.de, $+498153281383,+498153281445$
}

\begin{abstract}
The creation of adequate image segments based on given reference segments is a method receiving some attention in certain academic fields concerned with image analysis, including Geographic Object Based Image Analysis (GEOBIA). Typically such a method involves using an efficient search algorithm to traverse the parameter space of the given segmentation algorithm. Spatial metrics comparing generated and reference segments are normally used to direct the search process.

An extension to this general approach is investigated in this contribution where user generated reference segments are used in two distinct ways in the search process. Both the spectral and geometric aspects of the reference segments are queried and used within in the iterative optimization loop. A oneclass classification process using spectral information from inside the provided reference segments is used to generate a probability image, which in turn is employed to direct a modification of the original input imagery. Segmentation is performed on such a modified image. Initial results employing the proposed method are presented, comparing it to the standard search centric sample supervised segment generation approach in terms of segmentation quality.
\end{abstract}

Keywords: Image Segmentation, Classification, Data Transformation, Segmentation Metrics

\section{Introduction}

Search centric sample supervised segment generation denotes a general approach for the creation of image segments, to be used in further image analysis, based on the use of efficient search algorithms and segmentation quality measures (Bhanu et al., 1995; Freddrich and Feitosa, 2008). Such an approach shows promise in the discipline of GEOBIA, especially in the context of rule set approaches to image analysis that typically involve a substantial amount of parameter tuning.

A new variant of search centric sample supervised segment generation is presented that suggests the use of additional processes in the optimization loop to generate improved segmentation results. The contribution lies in suggesting the use of spectral information contained inside the user provided reference segments to tailor (transform) the data for the given problem. Process interdependencies are acknowledged (Fourie and Schoepfer, 2014), suggesting the creation of enlarged search spaces. From a user perspective, such an approach requires no additional steps compared to the standard variant of sample supervised segment generation; it is simply suggested to use more of the information that a user provides.

\section{Method}

Fig. 1 illustrates the proposed approach to segmentation algorithm parameter tuning. Firstly, the spectral data contained within the given reference segments are collected and used in a classification 
process. The resulting pixel probability image is integrated, in select ways, with the original input image to create a new modified image. This modified image is used as input to the given segmentation algorithm. Secondly, as with many of the variants of this general method, the spatial properties of the reference segments are compared to that of the generated segments to calculate a measure of quality. The core idea is that an initial pixel probability classification is used to tailor the data in such a way that the given segmentation algorithm could perform better on the given problem. This process differs from an unsupervised data transformation (Fourie and Schoepfer, 2014) as data samples are used to direct a change.

The proposed method has three aspects, or parameter domains that need consideration: The generation of the probability image (choice of classifier, parameters, sampling), integrating the probability and input images (strategy, parameters) and the tuning of the segmentation algorithm. A variant of this approach is demonstrated using a one-class (for masking) and a two-class (for classification) Support Vector Machine (SVM) to generate a probability image. The probability image is used to modify the original input image by moving the spectral values of the image to the average of reference segments based on the probabilities in the probability image. Various other integration strategies are also possible. This modified image is segmented with a segmentation algorithm (Baatz and Schäpe, 2000). Segment results are compared with the reference segments using the Reference Bounded Segments Booster (RBSB) (Freddrich and Feitosa, 2008) and the Reference Weighted Jaccard (RWJ) (Fourie and Schoepfer, 2014) metrics.

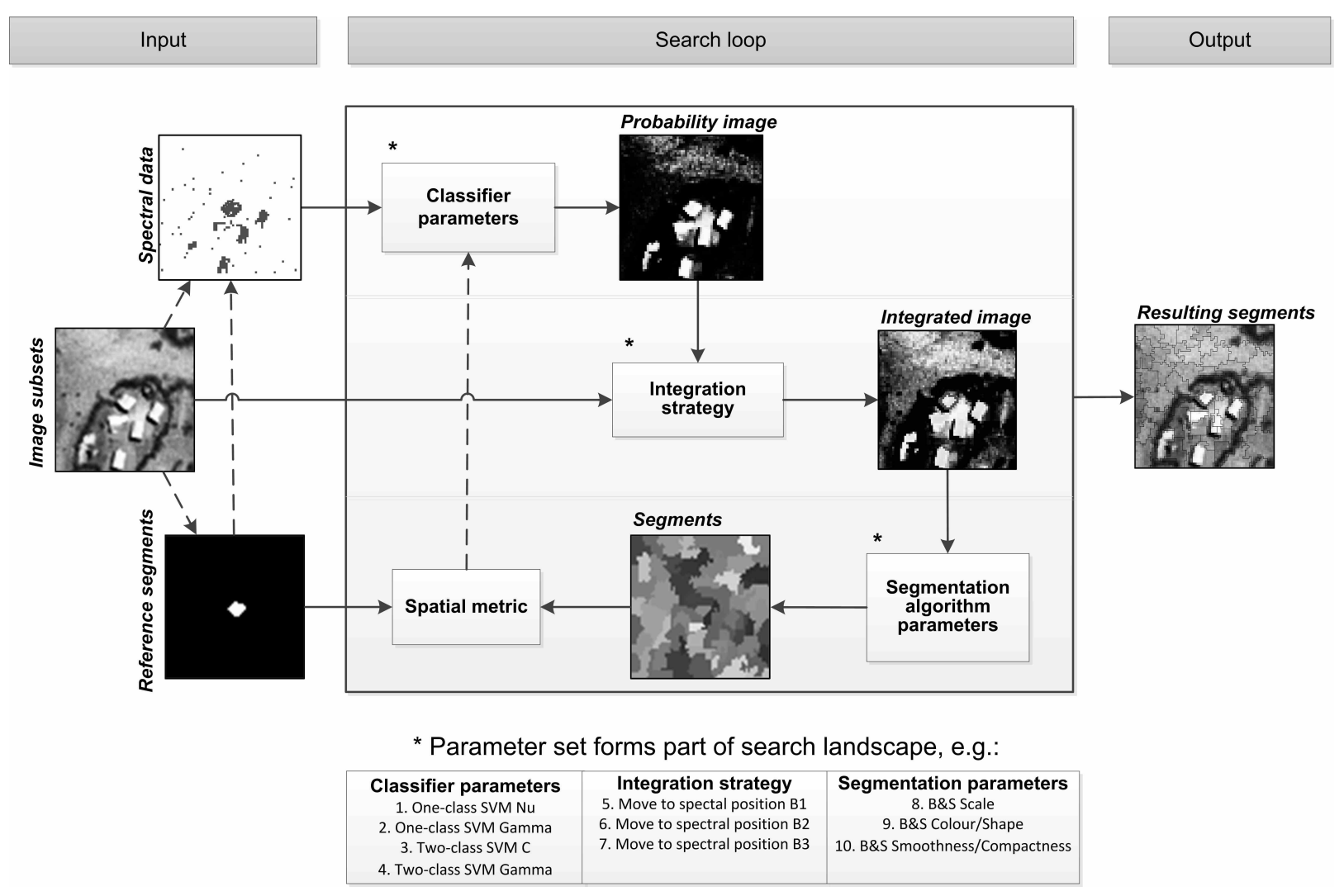

Figure 1: The architecture of the sample supervised search centric segmentation generation approach incorporating the use of the spectral data from the reference segments. 
Fig. 2 illustrates the utilized data (GeoEye-1, 0.5m spatial resolution resampled to $1 \mathrm{~m}$ in the processing system, channels 1,2,3) (C GeoEye, Inc. 2011, provided by e-GEOS S.p.A. under GSCDA, all rights reserved). The aim could be the identification of permanent structures. 17 Reference segments were digitized and used as input to the method, illustrated with the blue polygons in Fig. 2.

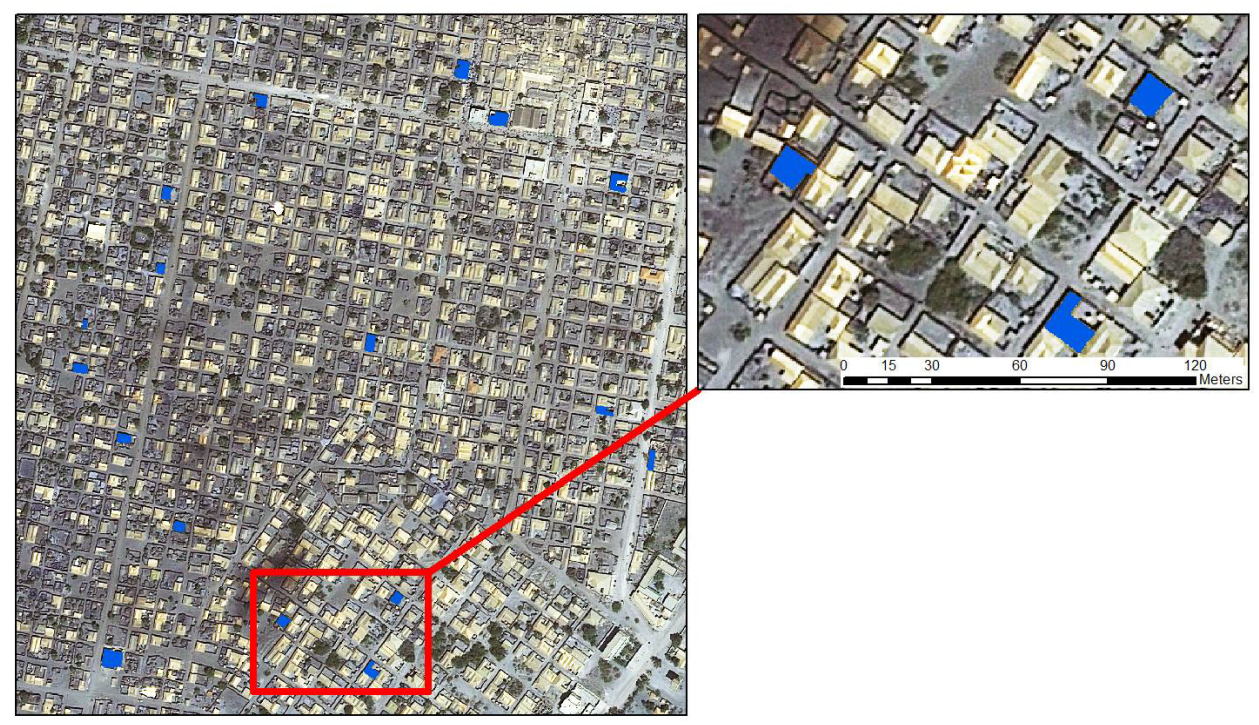

Figure 2: Utilised test dataset consisting of 17 reference segments. The aim could be to identify and characterize buildings.

\section{Results and discussion}

Table 1 lists the achieved metric scores (averaged over five runs) of a few variants of the proposed method tasked with segmenting buildings. The method was run by only tuning segmentation algorithm parameters, by tuning the generation of probabilities and segmenting on a probability image and with two strategies that generate modified imagery by combining the probability imagery with the original input imagery (details to be given in future work). The Differential Evolution metaheuristic was used. Lower metric scores signify better results.

Table 1: RBSB and RWJ metric scores for two variants of the proposed approach, as well as the standard variant and a variant including unsupervised data transformations.

\begin{tabular}{|l|l|l|l|l|}
\hline & $\begin{array}{l}\text { Parameter } \\
\text { Tuning }\end{array}$ & $\begin{array}{l}\text { Probability Tuning } \\
+ \text { Parameter Tuning }\end{array}$ & $\begin{array}{l}\text { Probability Tuning } \\
+ \text { Exchange band 2 } \\
+ \text { Parameter Tuning }\end{array}$ & $\begin{array}{l}\text { Probability Tuning } \\
\text { + Move to Average } \\
\text { + Parameter Tuning }\end{array}$ \\
\hline RWJ & 0.27 & 0.22 & 0.19 & 0.18 \\
\hline RBSB & 0.26 & 0.21 & 0.19 & 0.20 \\
\hline
\end{tabular}

The variants conducting some form of probability- and original image hybridisation delivered the best results as measured by the RWJ and RBSB metrics. Even segmenting on a probability image (adding four dimensions to the search landscape) is useful, compared to only tuning segmentation algorithm parameters. No extra processes from the user's perspective are needed with this strategy compared to the Parameter Tuning strategy. Additional computing time is required though for the added intermediary steps. Fig. 3 illustrates such intermediary steps (Fig. 3(b-e)) performed on the 
original input data (Fig. 3(a)) before segmentation is performed. Fig. 3(f) illustrates the best segmentation results obtained with the Probability Tuning + Move to Average + Parameter Tuning method variant.
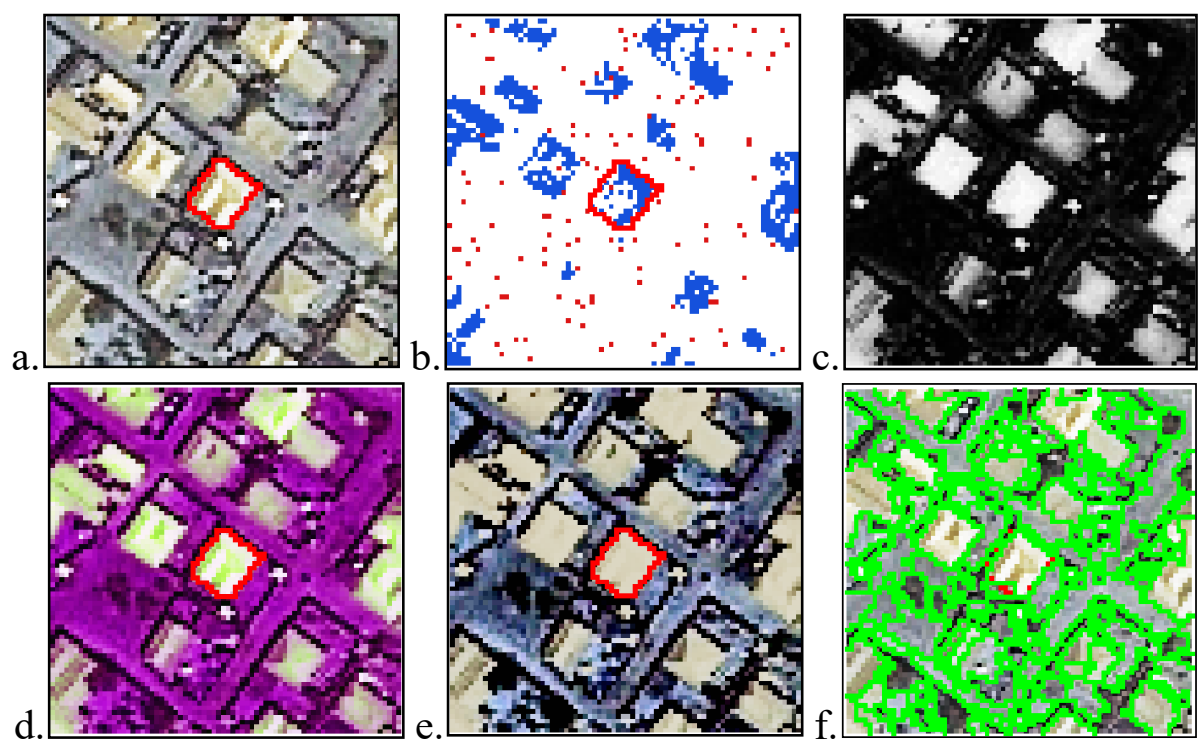

Figure 3: Illustration of intermediary processes of the proposed method. (a) Shows the original image (subset) and reference segment delineated with a red polyline, (b) a masking and sample gathering process (one-class SVM), (c) probability image (two-class SVM), (d) modified image by exchanging bands, (e) modified image by moving to average values and finally, (f) segment results with the best performing variant (Move to Average).

\section{Conclusion}

A search centric sample supervised segment generation method was demonstrated that modifies data in a supervised fashion to allow stronger spectral and thematic correlations. This assists the given segmentation algorithm on the given information extraction problem. Initial experimentation suggests merit of such an approach to improve results in situations where segmentation could be considered feasible a priori, but on difficult problems more elaborate image analysis strategies would be needed. Various variants of this approach are considered for further research, including using highly modular segmentation methods.

\section{Acknowledgements}

This work has been conducted under the GIONET project funded by the European Commission, Marie Curie Programme, Initial Training Networks, Grant Agreement number PIT-GA-2010-264509.

\section{References}

Baatz M., Schäpe A., 2000, Multiresolution segmentation: an optimization approach for high quality multi-scale image segmentation. In Angewandte Geographische Informationsverarbeitung, Strobl J., Blaschke T., Griesebner G. (editors), Karlsruhe, Germany, Wichmann Verlag. 
Bhanu B., Lee S., Ming J., 1995, Adaptive image segmentation using a genetic algorithm. Systems, Man and Cybernetics, IEEE Transactions on, Volume 25, Issue 2: 1543-1567.

Fourie C., Schoepfer E., 2014, Data transformation functions for expanded search spaces in geographic sample supervised segment generation. Remote Sensing, Submitted.

Freddrich C.M.B., Feitosa R.Q., 2008, Automatic adaptation of segmentation parameters applied to non-homogeneous object detection. Proceedings Geographic Object-based Image Analysis (GEOBIA 2008) Conference, Calgary, Canada. 


\section{APPENDIX B - SOFTWARE OVERVIEW}

\section{Introduction}

During this work experimental software was developed. It is not commercial grade software and was initially developed only to run experiments for the various chapters. The experiments presented in this work may be duplicated with the software without any programming knowledge. With some programming knowledge, the methods may be extended/elaborated upon or integrated in various other programs or research endeavours. For each presented method variant a separate program was created. The code will be made available upon request (via google drive - email to: fourie.christoff@gmail.com). If found useful, please cite the applicable article.

Other works are also available conducting automated segmentation algorithm parameter tuning. The Segmentation Parameter Tuner (SPT) (P. Achanccaray, V. Ayma, L. Jimenez, S. Garcia, P. Happ, R. Feitosa and A. Plaza, A free software tool for automatic tuning of segmentation parameters, SouthEastern European Journal of Earth Observation and Geomatics, 3, 707-712, (2014)) functions with the generic method variant and includes a substantial selection of segmentation algorithms, metrics and search methods. The Geographic Object Novelty Detector (Geo-ND) (Fourie, C. A one class object based system for sparse geographic feature identification. Stellenbosch University, Stellenbosch, 2011) also conducts basic parameter tuning and includes supervised classification mechanisms (also optimized with metaheuristics).

\section{Architecture}

The programs were developed in $\mathrm{C}++$, specifically in the Microsoft Visual $\mathrm{C}++$ IDE (and Microsoft $\mathrm{C}++$ compiler). Various open source libraries were used. The backend or base for image processing is OpenCV. Terralib is used for some processing, but primitive datatypes remain OpenCV Mat. Qt is used for the GUI and rendering windows. Mat datatypes are converted to Qt datatypes during rendering phases within the software. Other utilised C++ libraries include SwarmOps, LibSVM, Shark and various other bits.

Figure 1 illustrates the general layout of the software. It consists of two windows. A main processing window, in this instance entitled "Expanded search spaces" and a secondary window giving an overview of the loaded image, namely "Image overview". The image overview window also shows rectangles around reference segments that a user provided. Digitising may be done in any image processing software. The red rectangle denotes the currently selected reference segment. It is displayed in greater detail in the main program window ("Expanded search spaces"). 

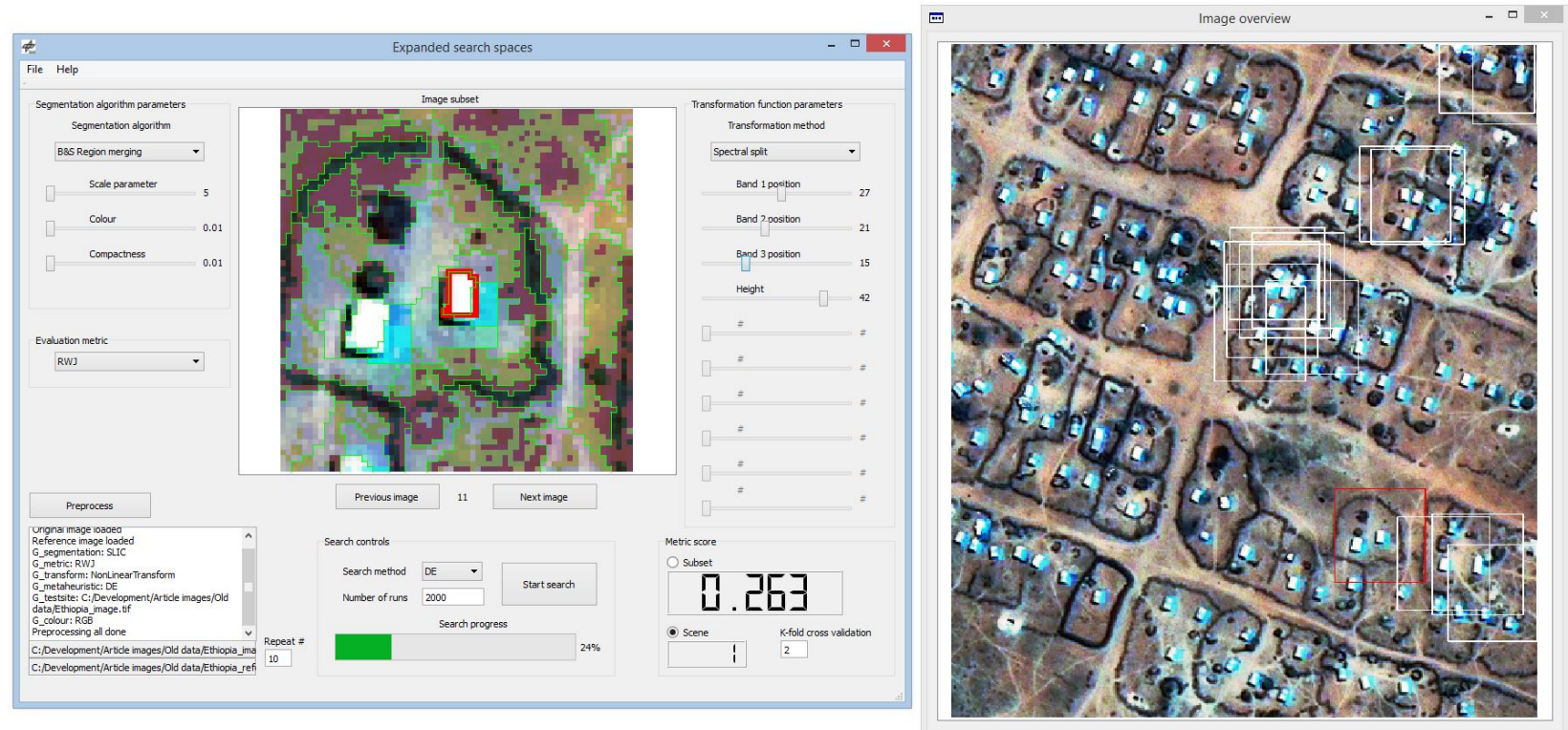

Figure 1: General GUI layout of the presented software. The software consists of two windows, one presenting controls and giving a preview of results on a given subset (changeable) and a window giving an overview of the entire image loaded.

\section{User Guide}

The software takes as input two imagery of exactly the same size. An image need to be loaded (3band, 8-bit, TIFF) by clicking "File" and "Load image". A mask also needs to be loaded representing the reference segments provided by an analyst. The mask file needs to be one band, 8-bit, TIFF. A value greater than one implies a pixel as part of a reference segment (default is 255). All over values should be 0 . Figure 2 gives an example (synthetic image), with the image on the left denoting the image of interest and the image on the right denoting a mask of reference segments - in this instance the four "structures".
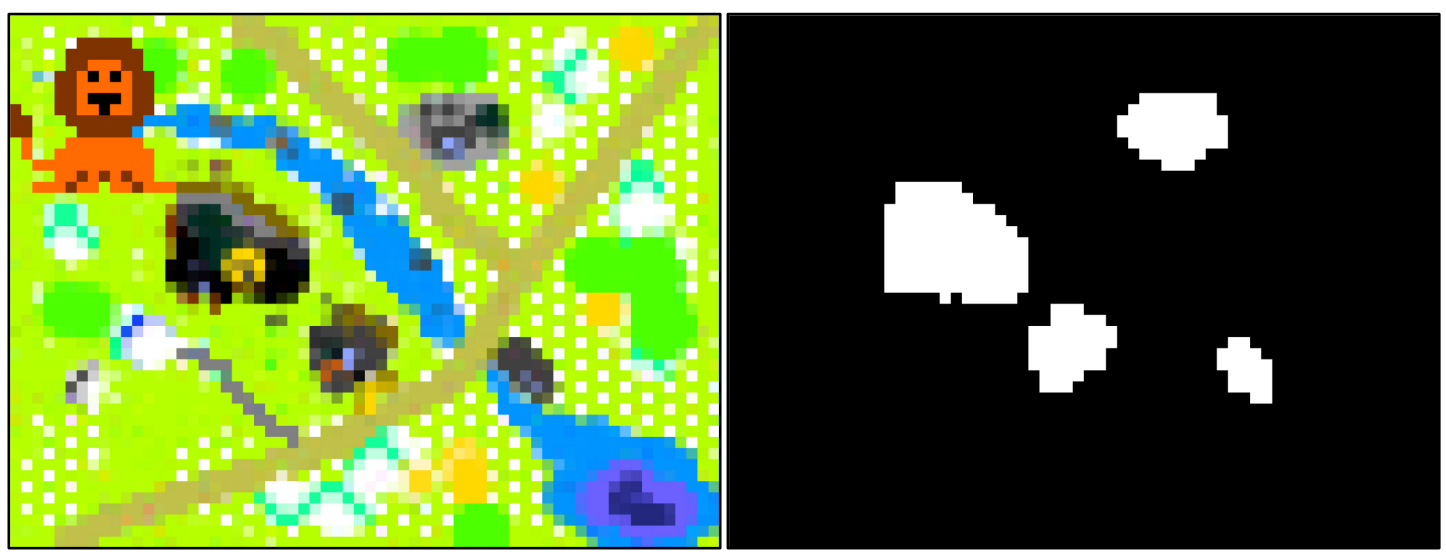

Figure 2: An example of an image of interest and the accompanying mask for the reference segments. 
After two images have been loaded the "Preprocess" button should be pressed (Figure 1). Various data management tasks are conducted. The box in the bottom left corner, the status or report box, will report on these processes and inform the user when pre-procesing is complete. The software is then primed for manual, automatic or combined operation.

Figure 3 illustrates the main program window in more detail. Controls are grouped by bounding boxes. On the left there is a set of controls to select and tune a given segmentation algorithm. There is also a dropdown box to change the used metric. Below the image are controls to change which reference segment is displayed. On the right are controls to select and tune a given data mapping function. Under "Search controls" one may set the used metaheuristic and start the automatic fitness landscape search process (progress bar will indicate progress). Under "Metric score" one may select local or global optimisation and define the magnitude of cross-validation. A "Repeat \#" box allows setting the number of experimental runs that need to be conducted. All results are presented in separate text files generated within the same folder as the executable. Settings are encoded within the text filenames, e.g.:

"Results_GeneticTransform_RWJ_BS_DE.txt"

"FitnessTrace_LinearTransform_RWJ_BS_DE_16.txt"

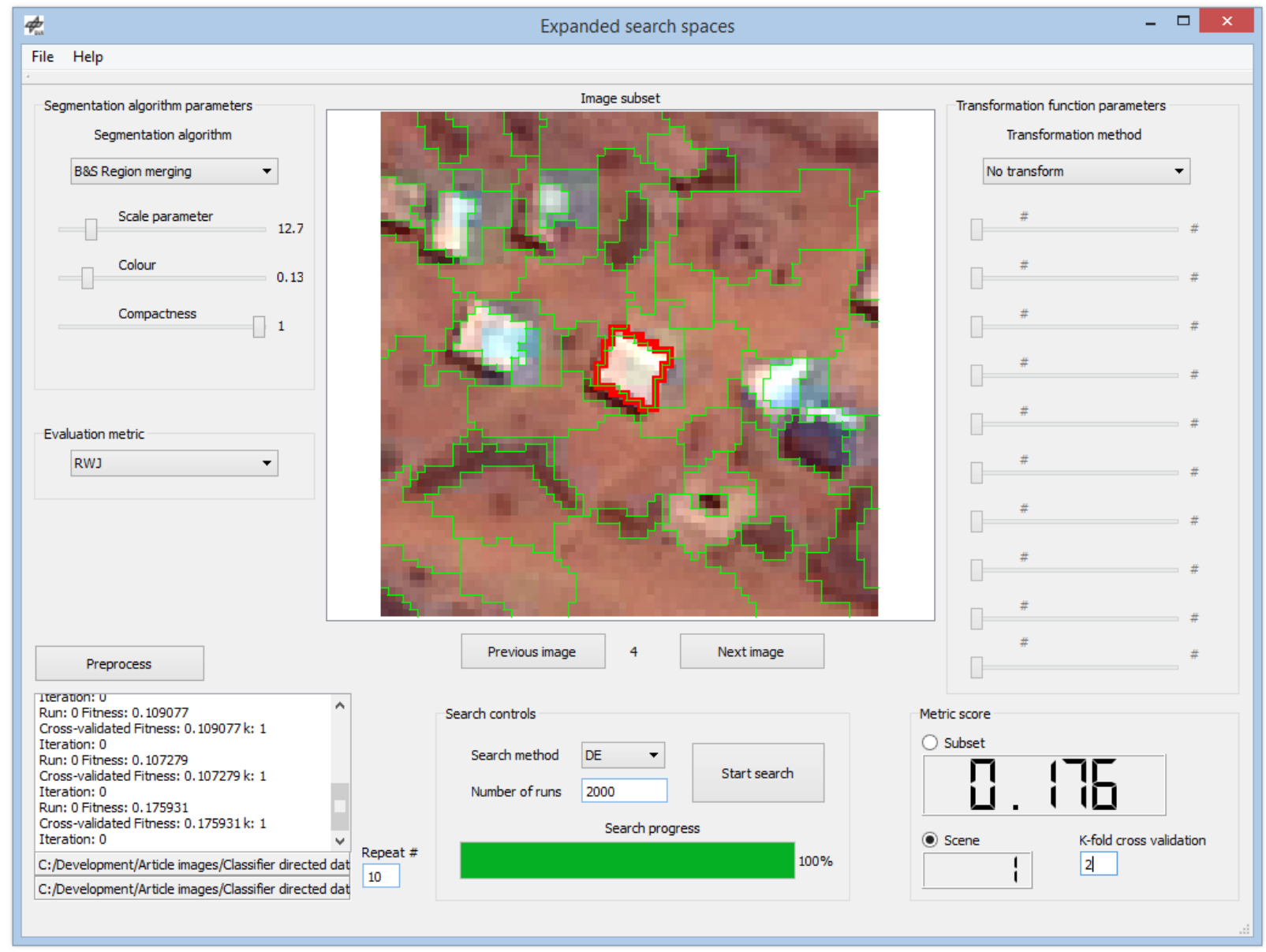

Figure 4: The main program window in more detail. 
Note that a user may interactively page through different reference segments and manually change parameters before or after an automatic search process. During the automatic search process the controls are unresponsive (program crash). Figure 5 shows the same window with the Genetic Transform mapping function selected. It is very interesting to alternatively tune the mapping function parameters and the segmentation algorithm parameters, noticing their interplay. The "Subset" metric score will update interactively, reflecting segment quality on the central reference segment as one alters the various parameters of the mapping function or segmentation algorithm.

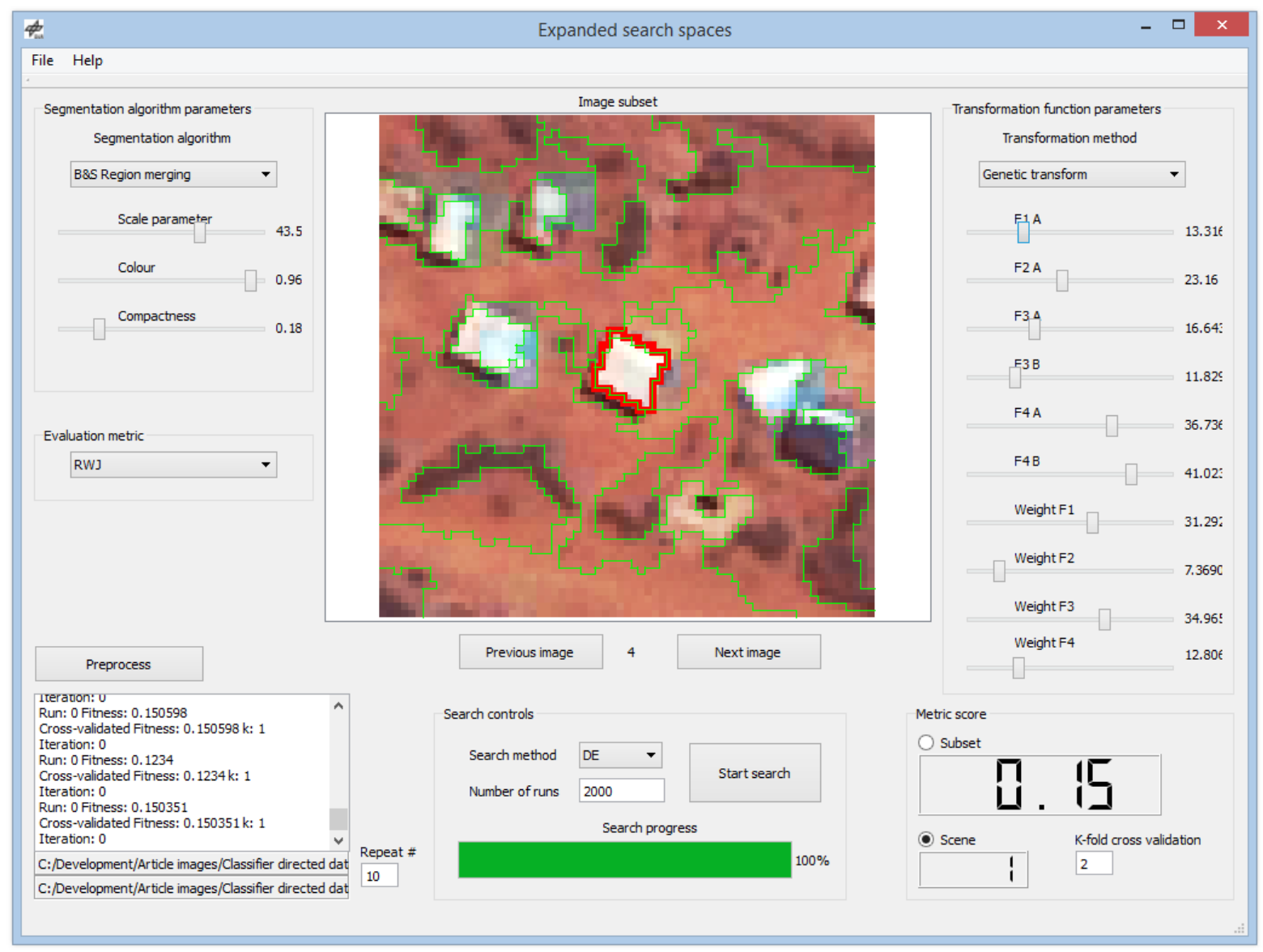

Figure 5: A mapping function selected with its parameters manually tuned.

The above figures represent the program developed for Chapter 3, where additional data mapping functions are considered as part of the fitness landscape. Figure 6 illustrates the program variant developed for graph based segmentation and attribute tuning. It is similar to the one developed for data mapping functions. In this variant the mapping function parameters may not be tuned manually (from inside the code it may), but are displayed in the textbox on the right. The bounding box on the left indicate the parameters of the $\mathrm{CC}$ segmentation algorithm, along with the thresholdable attributes. Disabling attributes increases the execution speed of the segmentation algorithm.

The final variant of the software includes classification processes for segmentation. During the development a very simple extension was made to allow rudimentary image classification (just for illustration of a more complete process - albeit extremely simple). Figure 7 illustrates the main window of the variant incorporating classifier directed data modification. Note that in this program variant there are three preview windows illustrating results of intermediary steps. The first window 
illustrates pixel sampling, the second window probability classification and the third window segmentation results on hybridized imagery. The small window on the left illustrates the entire image under consideration.

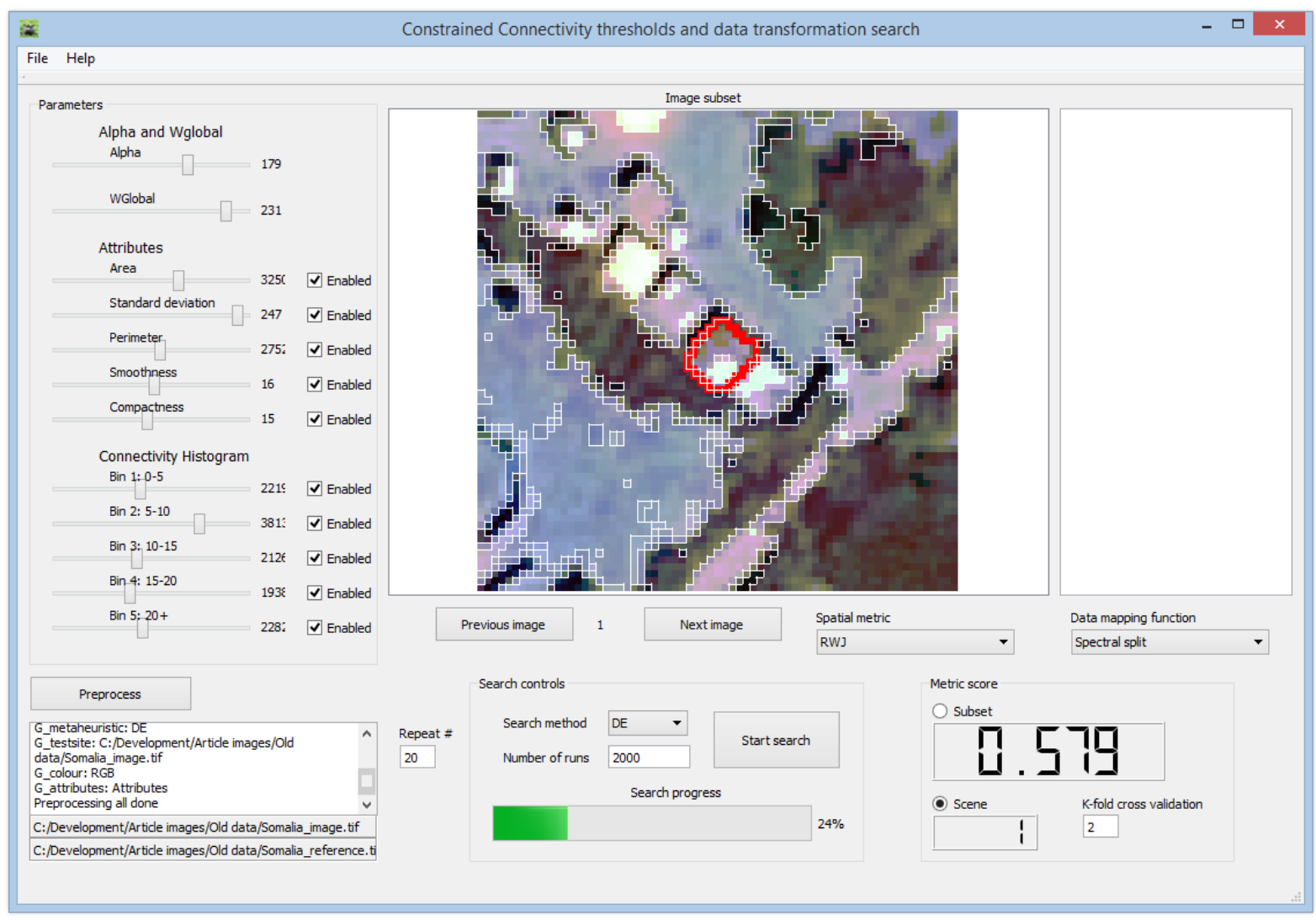

Figure 6: The program variant considering CC segmentation with additional attributes and mapping functions.

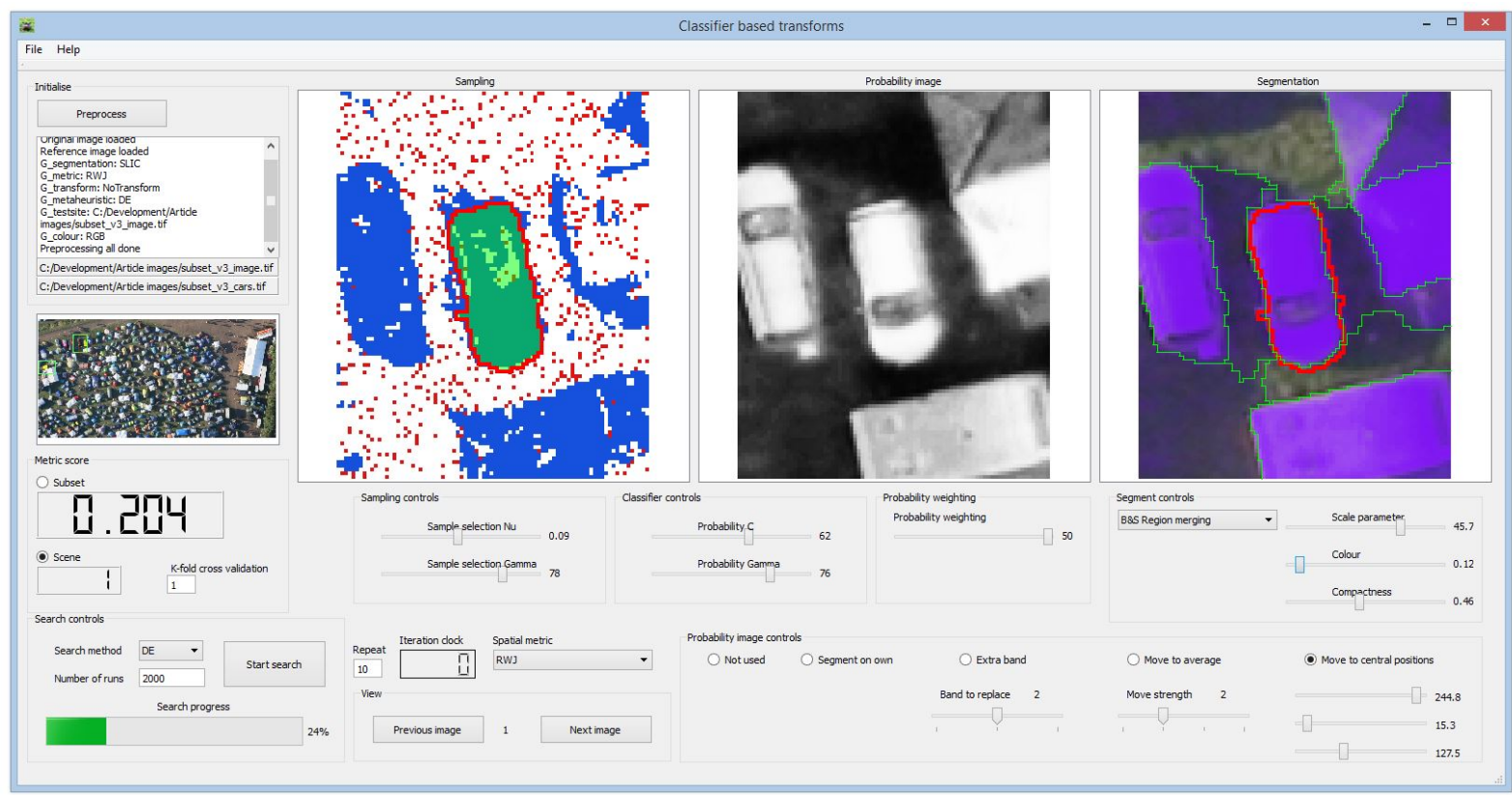

Figure 7: The program variant considering classifier directed data modification. 
The accompanying overview window for this method variant has been reworked to include oneclass SVM classification. Figure 8 shows this modification. After optimal segmentation parameters have been achieved, an analyst may perform a full classification in this window. On the left (Figure 8) are basic controls to segment the entire image, alter display properties and manually tune one-class SVM parameters.

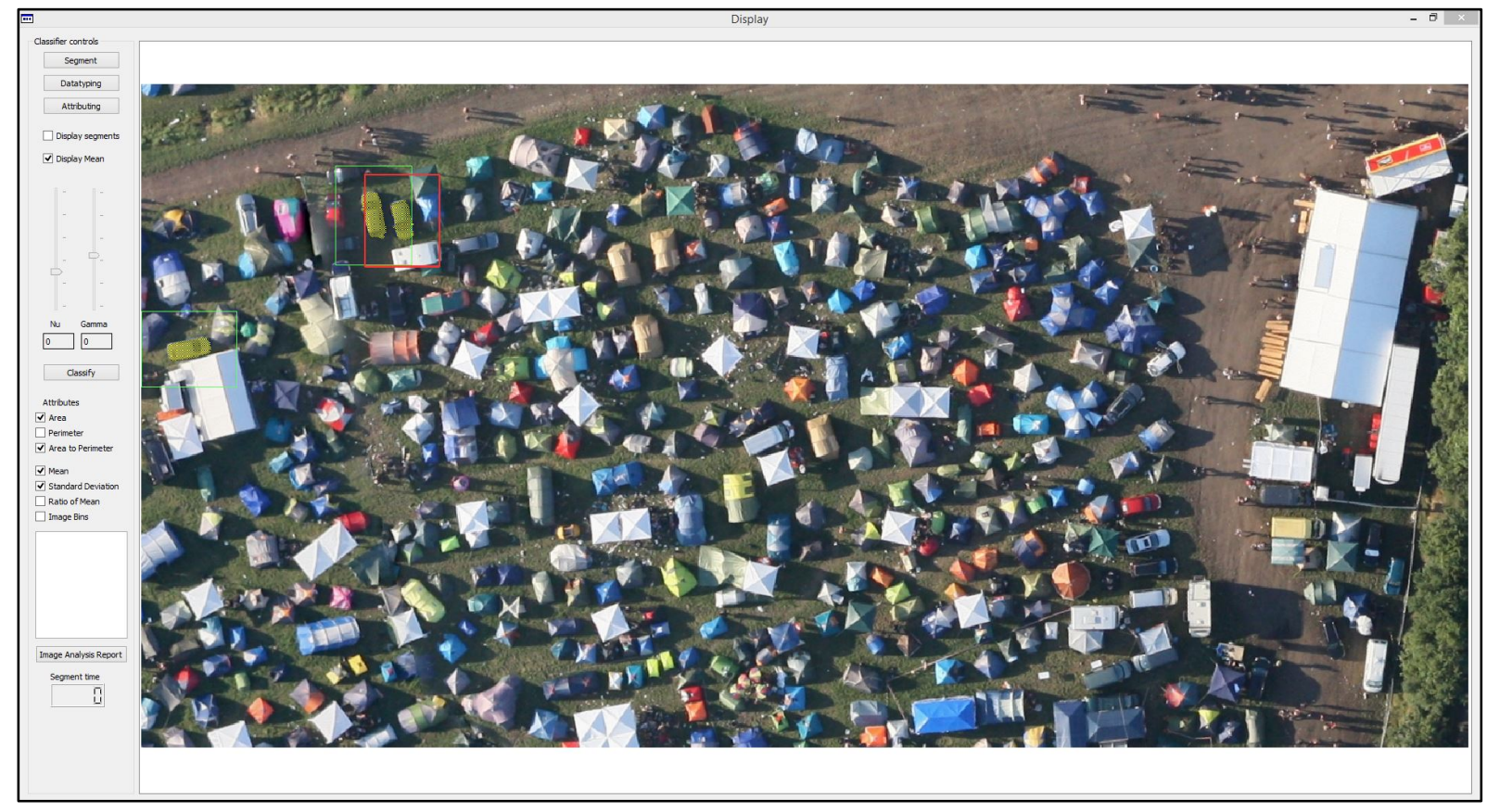

Figure 8: The overview window for the method variant incorporating classification in segmentation. As an additional exercise image classification was incorporated with this variant, implemented in this overview window.

Additional segment samples for classification may be selected on the image if the "Display segments" or "Display mean" options are ticked (left clicking). Figure 9 illustrates a basic example of using this tool to quickly find refugee tents. Two minutes of manual work was needed to select samples and tune parameters (it may be automated easily in the context of novelty detection). Alternatively, segments and mean spectral values may also be shown on the image (Figure 10). 


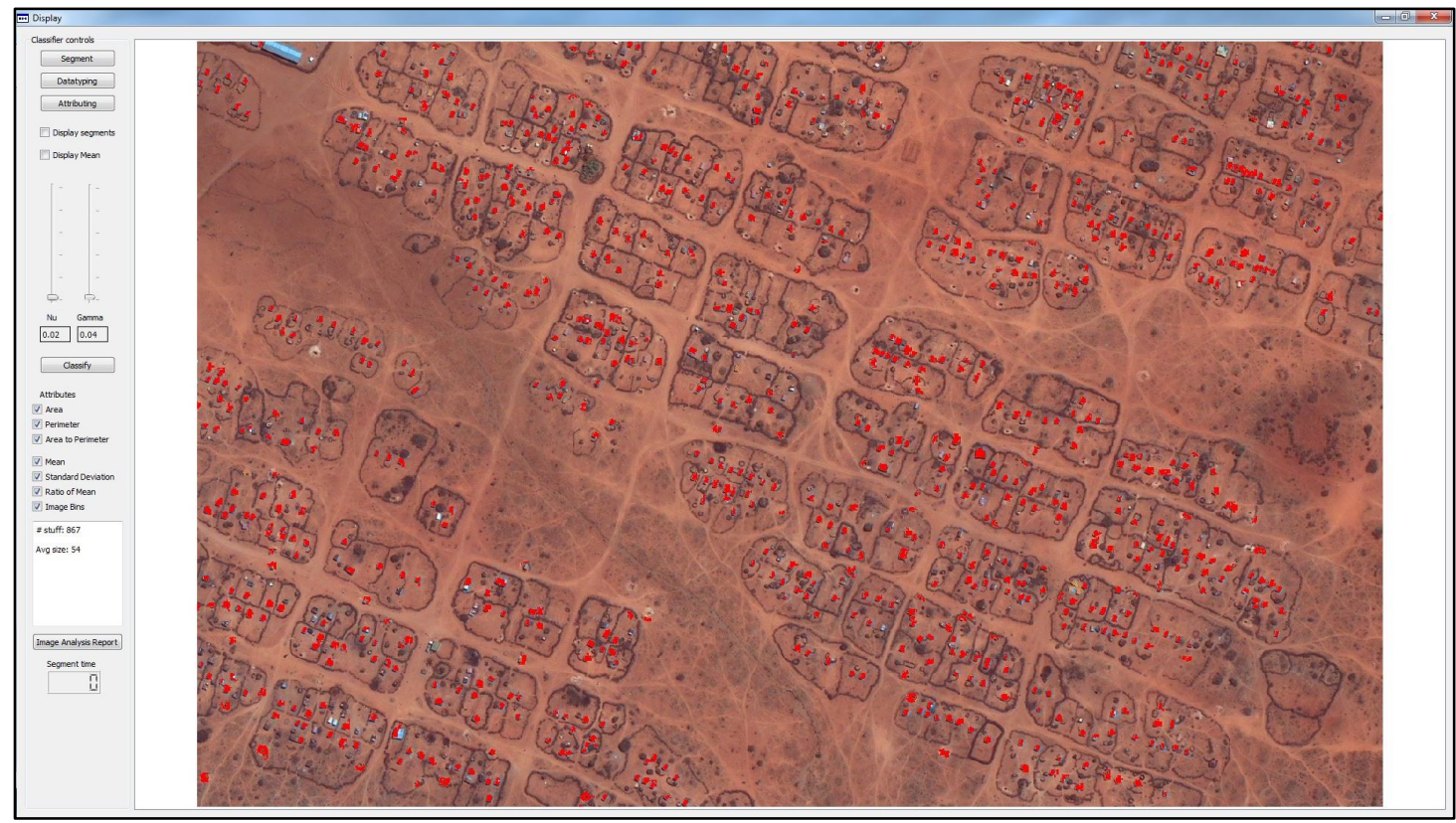

Figure 9: Example classification results from the classification tool used to find refugee tents.

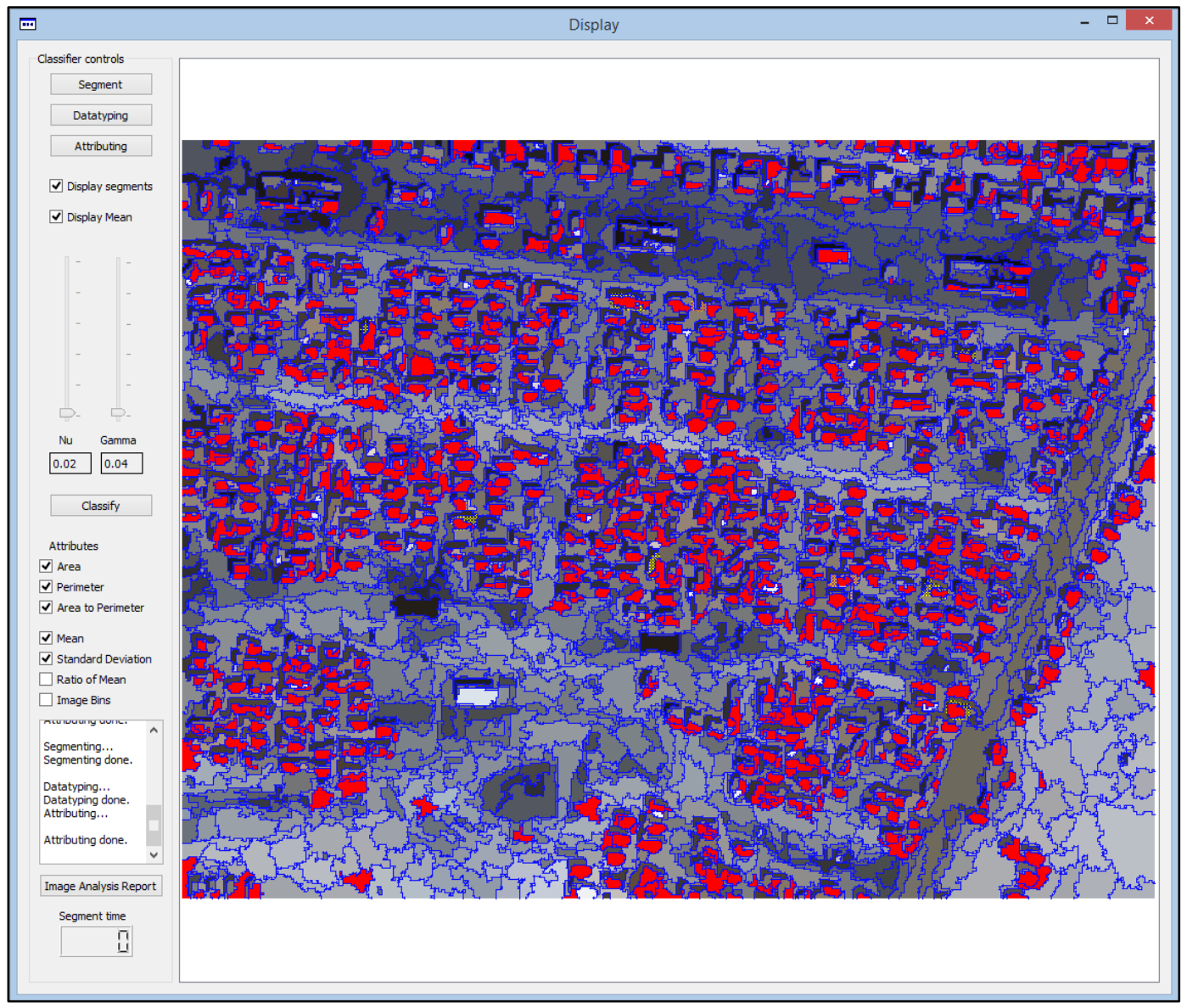

Figure 10: Example classification results also showing mean segment spectral values and segment borders. Note this is the same image as shown in the introductory chapter - no segment optimisation was conducted. 


\section{APPENDIX C - PARAMETER STANDARD DEVIATIONS}

In some results considering the Multiresolution Segmentation (MS) algorithm, parameter values are generated with very high standard deviations. This is due to the nature of the MS algorithm (see Appendix D). A range of parameter values were able to achieve the same segmentation results. The table below shows 23 runs for one of the many experiments conducted in Chapter 3. Two outliers were removed for illustrating correlations in this appendix. The results present segments generated for the Bokolmanyo dataset, with the RWJ metric. No data transformation/mapping was conducted.

Table 1: RWJ metric score values and generated MS algorithm parameters for an arbitrary problem from Chapter 3.

\begin{tabular}{|r|r|r|r|r|}
\hline \multicolumn{1}{|l|}{ Run } & RWJ score & Scale & Colour/Shape & Compactness/Smoothness \\
\hline 1 & 0.294834 & 20.4 & 0.259614 & 1 \\
\hline 2 & 0.280538 & 19 & 0.220534 & 0.780158 \\
\hline 3 & 0.275059 & 6.4 & 0.0210688 & 0.1182772 \\
\hline 4 & 0.276862 & 16.2 & 0.1363706 & 0.967018 \\
\hline 5 & 0.294833 & 20.4 & 0.259248 & 1 \\
\hline 6 & 0.301159 & 5 & 0 & 0.0509974 \\
\hline 7 & 0.285905 & 12 & 0.0740898 & 0.54747 \\
\hline 8 & 0.298802 & 14.8 & 0.1133496 & 0.780804 \\
\hline 9 & 0.286091 & 20.4 & 0.259752 & 1 \\
\hline 10 & 0.290989 & 13.4 & 0.0923572 & 0.693924 \\
\hline 11 & 0.299751 & 20.4 & 0.257462 & 0.989552 \\
\hline 12 & 0.26828 & 16.2 & 0.1365268 & 0.966024 \\
\hline 13 & 0.283697 & 13.4 & 0.0927534 & 0.624126 \\
\hline 14 & 0.285663 & 16.2 & 0.1357366 & 0.961508 \\
\hline 15 & 0.267932 & 13.4 & 0.0933846 & 0.702832 \\
\hline 16 & 0.279395 & 5 & 0 & 0.0491972 \\
\hline 17 & 0.295836 & 13.4 & 0.089889 & 0.676408 \\
\hline 18 & 0.294894 & 5 & 0 & 0.050198 \\
\hline 19 & 0.275157 & 5 & 0 & 0.0486048 \\
\hline 20 & 0.291018 & 12 & 0.0731132 & 0.536958 \\
\hline 21 & 0.283697 & 13.4 & 0.0931406 & 0.0489822 \\
\hline 22 & 0.298007 & 5 & 0 & 0.0494242 \\
\hline 23 & 0.289508 & 5 & 0.09 & $0.58 \pm 0.38$ \\
\hline Average: & $0.29 \pm 0.01$ & $12.67 \pm 5.71$ & $0.10 \pm 0.4$ & \\
\hline & & & & 0 \\
\hline
\end{tabular}

Note the very precise RWJ values $( \pm 0.01)$, with some variation in the three parameters of the MS algorithm. This is not a problem; it just means that if multiple runs are conducted that parameter averages may not be used. Simply, one may identify significant clusters in such a scenario and use those values or derive values based on correlations present within the results, illustrated with the three figures below (based on the above table). Such a strong correlation may not always be present, though. SLIC and CC segmentation did not display this behaviour under simple conditions. Note that adding additional fitness landscape constituents may exacerbate this consideration. 


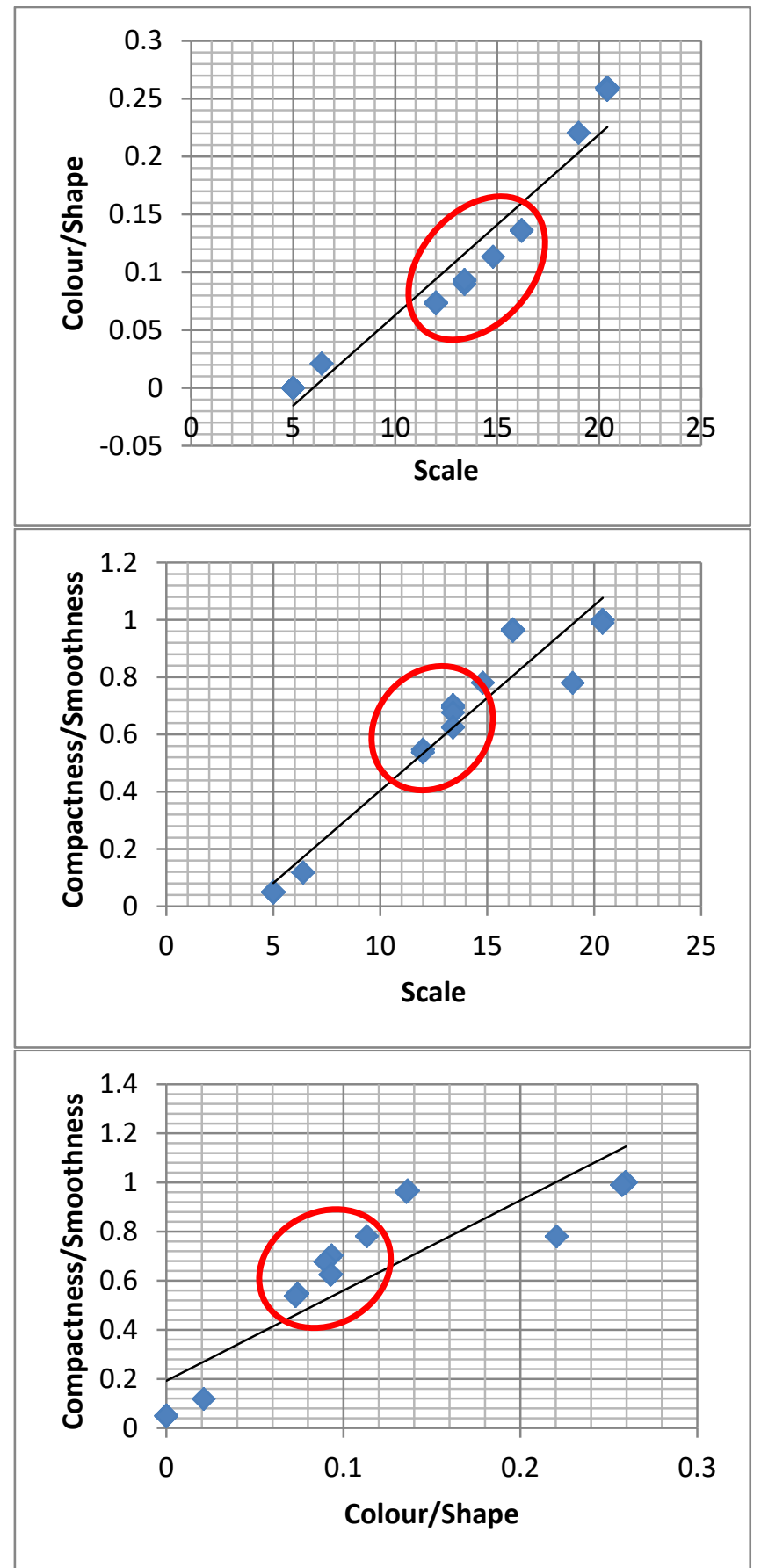

Figure 1: Correlation plots of the three parameters of MS for the results from the table above. Suitable parameters may be derived by identifying commonly generated parameter sets (clusters - red ellipsoids) or by using information derived from the correlation (not necessarily present in all problems). 


\section{APPENDIX D - ALGORITHM DETAILS}

\section{Appendix D.1: Differential Evolution}

The Differential Evolution (DE) metaheuristic is used throughout this dissertation as the main optimisation method. Initial experimentation in this work was conducted with a new variant of cuckoo search [1], programmed from scratch. It was outperformed by both classical DE and classical PSO (SwarmOps library [2]) on some test problems in the context of this work and relegated to merely illustrating fitness landscapes (Chapter 1). DE had the overall best performances and was thus subsequently used.

$\mathrm{DE}$ is one of the classical and very well performing variants of continuous space population based metaheuristics. The body of literature on DE is rich, with the seminal paper [3] enjoying around 9500 citations at the time of writing this manuscript. See [4] for a recent review on research in the context of DE. Here the classical ' $\mathrm{DE} / \mathrm{rand} / 1 /$ ' variant of $\mathrm{DE}$ is employed. As with most metaheuristics, a population of agents are defined (NP denote the number of agents), undergoing mutations in generations $(\mathrm{G})$. An agent, $\mathrm{X}$, for a specific generation may thus be denoted as:

$$
X_{i, G}, i=1,2, \ldots, N P
$$

where $\mathrm{i}$ denote entries in the solution space (solution vector or parameters to a method). During the execution of $\mathrm{DE}$ an agent $(\mathrm{X})$ may be replaced by a trail agent (U) if the fitness of the trail agent is lower (or higher) than the agent under observation. A trail agent is generated (crossover) by selecting solution vector elements from either the agent under observation or a mutant agent $(\mathrm{V})$ via:

$$
U_{j}=\left\{\begin{array}{l}
V_{j} \text { if }(\text { rand } \leq C R) \text { or } j=r n b r \\
X_{i} \text { if }(\text { rand }>C R) \text { and } j \neq r n b r
\end{array}\right.
$$

Where rand is a random number in the range [0..1] and rnbr a randomly chosen solution vector element guaranteed to be modified. CR is the crossover constant and is a user defined variable ([0..1]).

$\mathrm{V}$, the mutant agent, is calculated as follows:

$$
V=X_{a}+F \times\left(X_{b}-X_{c}\right)
$$

Where $\mathrm{X}_{\mathrm{a}-\mathrm{c}}$ are three random agents selected from the current generation (hence the name "Differential Evolution"). $\mathrm{F}$ is another user defined parameter ([0..2]) influencing the trade-off between exploitation (small F) and exploration (large F). The review article [4] provides excellent insight into why DE is effective and so popular. 


\section{Appendix D.2: Multiresolution Segmentation}

Multiresolution Segmentation (MS) [5] is arguably the best known segmentation algorithm within the GEOBIA community. This may be due to its good general performance in the context of VHR optical data. It is the main segmentation algorithm advocated in the most prominent commercial software available in the field. This may attribute to its popularity (something that should be noted the exact formulation in said software remains unclear). Here a variant is used, found within the excellent Terralib library. The description here is a reflection of the formulation given in [5].

Multiresolution segmentation is a region merging algorithm; meaning pixels are merged forming segments, with these segments merged and so on until certain threshold conditions are reached preventing segment growth. Critical in this process is the degree of fitting, or the similarity of a potential merge of two segments. For MS it is defined, considering only mean spectral content, as:

$$
h=\sqrt{\left(\frac{f_{1}-f_{2}}{\sigma}\right)^{2}}
$$

where $f_{1}$ and $f_{2}$ are the mean spectral values of candidate segments. $\sigma$ is a normalisation factor, namely the standard deviation of spectral values of all segments. Two segments are evaluated for a possible merge by calculating the change in the degree of fitting, given as:

$$
h_{\text {diff }}=h_{m}-\frac{h_{1}+h_{2}}{2}
$$

Where $h_{m}$ is the degree of fitting after a virtual merge and $h_{1}$ and $h_{2}$ the degree of fitting of the segments before the virtual merge. Object size is added as an additional measure to $h_{\text {diff }}$ resulting in:

$$
h_{\text {diff }}=\left(n_{1}+n_{2}\right) h_{m}-\left(n_{1} h_{1}+n_{2} h_{2}\right)
$$

where $\mathrm{n}$ are the object sizes of the respective segments under consideration. Two additional geometric heterogeneity measures are added, namely compactness and smoothness. Others may be considered also [6]. Compactness is defined as:

$$
h_{\text {compact }}=\frac{l}{\sqrt{n}}
$$

where 1 is segment boundary length. Smoothness is defined as:

$$
h_{\text {smooth }}=\frac{l}{b}
$$

where $\mathrm{b}$ denote the perimeter of the segment bounding box. The three heterogeneity measures are combined in a weighted fashion in the segmentation algorithm - constituting the parameters that need to be tuned. The Scale parameter defines relative segment sizes $\left(\mathrm{h}_{\text {diff }}\right)$. The Shape/Colour parameter is a weighting factor determining the importance of the Scale parameter versus the two geometric 
heterogeneity measures (range [0..1]). The Compactness/Smoothness parameter is a similar weighting parameter determining the importance between the two geometric heterogeneity criteria (again, other geometric criteria may be considered [6]).

Various strategies may be used to decide on the merging of segments. Here (in the Terralib implementation) a variant is employed using "best fitting", namely a segment is merged with a neighbour resulting in the best fit. A dither matrix is used to identify segments and ensures a distributed/ordered treatment. Alternatively, local or global mutual best fitting may be considered (slower - not considered in this work). Although MS is generally well received, see [7] for some interesting remarks about its limitations. 


\section{Appendix D.3: Simple Linear Iterative Clustering}

The Simple Linear Iterative Clustering (SLIC) algorithm [8,9] is considered a superpixel segmentation algorithm. Superpixel algorithms are typically much faster and simpler than algorithms traditionally found within remote sensing. They are not intended for semantic image segmentation, just to generate objects for the sake of having a richer attribute or feature set.

By default SLIC clusters pixels using a distance measure in the five-dimensional space consisting of a pixel colour vector in the CIELAB colour space and a pixel $\mathrm{x}$ and $\mathrm{y}$ position. In this work (remote sensing) three image input bands were always used, not necessarily in the RGB colour space. A conversion to CIELAB colour space was not conducted and SLIC was used with the default available bands (and x,y positions).

SLIC may be considered a special case of k-means clustering. Seed points are defined with a dither matrix. The algorithm defines windows around seed points, approximate to desired segment size (S) (double the distance of seed point spacing, 2S x 2S). Each pixel in a window (i) is assigned to a seed point with the lowest distance measure $(\mathrm{j})$. The distance measure, $\mathrm{D}$, may be written as:

$$
\begin{gathered}
d_{c}=\sqrt{\left(a_{j}-a_{i}\right)^{2}+\left(b_{j}-b_{i}\right)^{2}+\left(c_{j}-c_{i}\right)^{2}} \\
d_{s}=\sqrt{\left(x_{j}-y_{i}\right)^{2}+\left(y_{j}-y_{i}\right)^{2}} \\
D=\sqrt{d_{c}{ }^{2}+\left(\frac{d_{s}}{S}\right)^{2} m^{2}}
\end{gathered}
$$

Where $\mathrm{m}$ is a user defined weighting parameter defining the trade-off between spectral and spatial distance measures. a,b and c are colour space values. After an initial pass of the algorithm a new seed point is calculated for all segments. All segments are then re-evaluated for membership to new seed points. This process is repeated ten times, found sufficient by the authors in [8], resulting in the final segments.

\section{Appendix References}

1. Walton, S.; Hassan, O.; Morgan, K.; Brown, M. Modified cuckoo search: A new gradient free optimisation algorithm. Chaos, Solitons \& Fractals. 2011, 44, 710-718.

2. Pedersen, M. Swarmops: Black-box optimization in ansi c; Hvass Lab., Southampton, UK, 2008.

3. Storn, R.; Price, K. Differential evolution-a simple and efficient heuristic for global optimization over continuous spaces. Journal of global optimization. 1997, 11, 341-359.

4. Das, S.; Suganthan, P.N. Differential evolution: A survey of the state-of-the-art. Evolutionary Computation, IEEE Transactions on. 2011, 15, 4-31.

5. Baatz, M.; Schäpe, A. Multiresolution segmentation: An optimization approach for high quality multi-scale image segmentation. In Angewandte geographische informationsverarbeitung, Strobl, J.; Blaschke, T.; Griesebner, G., Eds. Wichmann Verlag: Karlsruhe, Germany, 2000; Vol. 12, pp $12-23$. 
6. Ferreira, R.S.; Feitosa, R.Q.; Costa, G.A.O.P. A multiscalar, multicriteria approach for image segmentation, Paper presented at Geographic Object-Based Image Analysis (GEOBIA 2012), Rio de Janeiro, Brazil, 7-9 May 2012.

7. Castilla, G.; Hay, G.J.; Ruiz-Gallardo, J.R. Size-constrained region merging (scrm): An automated delineation tool for assisted photointerpretation. Photogrammetric Engineering and Remote Sensing. 2008, 74, 409-419.

8. Achanta, R.; Shaji, A.; Smith, K.; Lucchi, A.; Fua, P.; Susstrunk, S. Slic superpixels compared to state-of-the-art superpixel methods. Pattern Analysis and Machine Intelligence, IEEE Transactions on. 2012, 34, 2274-2282.

9. Achanta, R.; Shaji, A.; Smith, K.; Lucchi, A.; Fua, P.; Süsstrunk, S. Slic superpixels; 2010. 


\section{AUTHOR CONTRIBUTIONS}

Erklärung zu den Eigenanteilen des Promovenden/der Promovendin sowie der weiteren Doktoranden / Doktorandinnen als Koautoren an den Publikationen und Zweitpublikationsrechten bei einer kumulativen Dissertation. 


\section{Article 1}

Fourie, C.; Schoepfer, E. Data transformation functions for expanded search spaces in geographic sample supervised segment generation. Remote Sensing. 2014, 5, 3791-3821.

German Remote Sensing Data Center (DFD), German Aerospace Center (DLR), 82234 Oberpfaffenhofen.

\begin{tabular}{|l|l|l|}
\hline Beteiligt an & Christoff Fourie & Elisabeth Schoepfer \\
\hline $\begin{array}{l}\text { Konzeption des } \\
\text { Forschungsansatzes }\end{array}$ & $\mathrm{X}$ & \\
\hline Planung der Untersuchung & $\mathrm{X}$ & \\
\hline Datenerhebung & $\mathrm{X}$ & \\
\hline Datenanalyse und interpretation & $\mathrm{X}$ & \\
\hline Schreiben des Manuskript & $\mathrm{X}$ & $\mathrm{X}$ \\
\hline $\begin{array}{l}\text { Vorschlag Anrechnung } \\
\text { Publikations-äquivalente }\end{array}$ & 1.0 & n.a. \\
\hline
\end{tabular}

\section{Article 2}

Fourie, C. On attribute thresholding and data mapping functions in a supervised connected component segmentation framework. Remote Sensing. 2015, 7, 7350-7377.

German Remote Sensing Data Center (DFD), German Aerospace Center (DLR), 82234 Oberpfaffenhofen.

\begin{tabular}{|l|l|}
\hline Beteiligt an & Christoff Fourie \\
\hline $\begin{array}{l}\text { Konzeption des } \\
\text { Forschungsansatzes }\end{array}$ & $\mathrm{X}$ \\
\hline Planung der Untersuchung & $\mathrm{X}$ \\
\hline Datenerhebung & $\mathrm{X}$ \\
\hline Datenanalyse und interpretation & $\mathrm{X}$ \\
\hline Schreiben des Manuskript & $\mathrm{X}$ \\
\hline $\begin{array}{l}\text { Vorschlag Anrechnung } \\
\text { Publikations-äquivalente }\end{array}$ & 1.0 \\
\hline
\end{tabular}




\section{Article 3}

Fourie, C.; Schoepfer, E. Classifier directed data hybridization for geographic sample supervised segment generation. Remote Sensing. 2014, 6, 11852-11882.

German Remote Sensing Data Center (DFD), German Aerospace Center (DLR), 82234 Oberpfaffenhofen.

\begin{tabular}{|l|l|l|}
\hline Beteiligt an & Christoff Fourie & Elisabeth Schoepfer \\
\hline $\begin{array}{l}\text { Konzeption des } \\
\text { Forschungsansatzes }\end{array}$ & $\mathrm{X}$ & \\
\hline Planung der Untersuchung & $\mathrm{X}$ & \\
\hline Datenerhebung & $\mathrm{X}$ & \\
\hline Datenanalyse und interpretation & $\mathrm{X}$ & \\
\hline Schreiben des Manuskript & $\mathrm{X}$ & $\mathrm{X}$ \\
\hline $\begin{array}{l}\text { Vorschlag Anrechnung } \\
\text { Publikations-äquivalente }\end{array}$ & 1.0 & n.a. \\
\hline
\end{tabular}

\section{Article 4 (book chapter)}

Fourie, C.; Schoepfer, E. Sample supervised search-centric approaches in geographic object-based image analysis (geobia): Concepts, state-of-the-art and a future outlook. In Earth observation for land and emergency monitoring - innovative concepts for environmental monitoring from space, Balzter, H., Ed. Wiley: in preperation.

German Remote Sensing Data Center (DFD), German Aerospace Center (DLR), 82234 Oberpfaffenhofen.

\begin{tabular}{|l|l|l|}
\hline Beteiligt an & Christoff Fourie & Elisabeth Schoepfer \\
\hline $\begin{array}{l}\text { Konzeption des } \\
\text { Forschungsansatzes }\end{array}$ & $\mathrm{X}$ & \\
\hline Planung der Untersuchung & $\mathrm{X}$ & \\
\hline Datenerhebung & $\mathrm{X}$ & \\
\hline Datenanalyse und interpretation & $\mathrm{X}$ & \\
\hline Schreiben des Manuskript & $\mathrm{X}$ & $\mathrm{X}$ \\
\hline $\begin{array}{l}\text { Vorschlag Anrechnung } \\
\text { Publikations-äquivalente }\end{array}$ & 1.0 & n.a. \\
\hline
\end{tabular}


Die Co-Autoren der in dieser kumulativen Dissertation verwendeten Manuskripte sind sowohl über die Nutzung als auch über die oben angegebenen Eigenanteile informiert und stimmen dem zu.

Ich bin mit der Abfassung der Dissertation als publikationsbasiert, d.h. kumulativ, einverstanden und bestätige die vorstehenden Angaben. Eine entsprechende begründete Befürwortung mit Angabe des wissenschaftlichen Anteils des Doktoranden / der Doktorandin an den verwendeten Publikationen werde ich parallel an den Rat der Fakultät der Chemisch-Geowissenschaftlichen Fakultät richten.

Name Erstbetreuer(in)

Name Zweitbetreuer(in)
Datum

Datum
Ort

Ort
Unterschrift 


\section{CURRICULUM VITAE}

\section{Christoffel Ettienne Fourie}

Age: 31

Gender: Male

Languages: Fluent in English and Afrikaans

Marital status: Unmarried

E-mail address: fourie.christoff@gmail.com

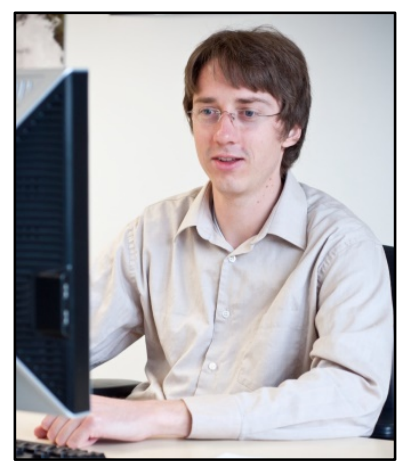

\section{Current affiliation}

A Marie Curie fellow finishing a doctorate in earth observation. I explore ways in which sample supervised geographic object based image analysis may be modelled as optimisation problems. This entails coming up with interesting interdependencies and creating prototype user driven image analysis software $(\mathrm{C}++)$.

\section{Education}

- Submission of P.hD. dissertation in July 2015 at Friedrich-Schiller University Jena, Germany.

- Research Masters in Geographic Information Science, Cum Laude (2011) at Stellenbosch University.

- Honours B.Sc. in Geographic Information Science, Cum Laude (2007) at Stellenbosch University.

- B.Sc. in Applied Geoinformatics (2006) focussing on computer science and geoinformatics at Stellenbosch University.

- Matriculated Cum Laude, South Africa (2002)

\section{Work experience}

- Researcher, German Aerospace Center (DLR), Oberpfaffenhofen, Germany (2011-2014): Research and development in the context of geographic object-based image analysis.

- GIS lab manager, Stellenbosch University, South Africa (2008): GIS support for researchers and students, IT support for staff and general lab management.

- Teaching assistant, Stellenbosch University, South Africa (2007): Geographic Information Systems $3^{\text {rd }}$ year, Digital Photogrammetry $3^{\text {rd }}$ year, Remote Sensing $3^{\text {rd }}$ year. 


\section{Professional skills}

- Researcher: I enjoy exploring the application and extension of basic research from computer science, machine learning and computer vision in a satellite remote sensing image analysis context. Technical research themes I work on include mathematical morphology inspired and classical image segmentation, metaheuristic applications, spatial quality metrics, classification, feature selection and general image processing.

- Programmer: Specialising within the domain of Geoinformatics. Familiar with common and uncommon open source libraries employed within Geoinformatics, including Terralib, Qt, LibSVM, OpenCV and SwarmOps. Building image/data analysis software is a major interest. See for example $\underline{A}$ one-class object-based system for sparse geographic feature identification (chapter four) and Data transformation functions for expanded search spaces in geographic sample supervised segment generation. I want to become skilful in ITK/OTB.

- Remote sensing specialist: Proficient in all aspects of image pre-processing, low and mid-level image processing and classification techniques. Familiarity with a range of application areas, including vegetation mapping, refugee camp monitoring and general land cover mapping.

\section{Technical skills}

- Preferred programming languages: $\mathrm{C}++$ and MATLAB but also familiar with Java, Visual Basic, SQL, Assembly and html/asp.

- Skilled in using: eCognition, ArcGIS, ERDAS, Geomatica, ENVI and IDRISI.

\section{Additional information}

- EC Marie Curie fellowship (http://www.gionet.eu). This entailed various summer schools, training, presenting and attending at various conferences (GEOBIA 2012, 2014, IGARSS 2012, 2013, GECCO 2013, 2014).

- LinkedIn profile (http://www.linkedin.com/in/cefourie)

- Google scholar (https://scholar.google.com/citations?user=N_TqHS4AAAAJ\&hl=en)

\section{Biography, personal interests and career goal}

I was fortunate to grow up in South African National Parks. I immensely enjoyed living near natural environments. Strangely enough I developed a keen interest in technology and computing. This balance shaped my interest in Geoinformatics. Some weekends and holidays are spent travelling (35 countries and counting). I want to position myself at the intersection of academia and industry as a Research and Development Scientist. I 
thoroughly enjoy all aspects of science ("finding the gap", writing, traversing disciplines), but enjoy building tools $(\mathrm{C}++)$ just as much.

\section{Publications}

Fourie, C.; Schoepfer, E. Sample supervised search-centric approaches in geographic object-based image analysis (geobia): Concepts, state-of-the-art and a future outlook. In Earth observation for land and emergency monitoring - innovative concepts for environmental monitoring from space, Balzter, H., Ed. Wiley: in preparation.

Fourie, C. On attribute thresholding and data mapping functions in a supervised connected component segmentation framework. Remote Sensing. 2015, 7, 7350-7377.

Fourie, C.; Schoepfer, E. Classifier directed data hybridization for geographic sample supervised segment generation. Remote Sensing. 2014, 6, 11852-11882.

Fourie, C.; Schoepfer, E. Data transformation functions for expanded search spaces in geographic sample supervised segment generation. Remote Sensing. 2014, 5, 3791-3821.

Fourie, C.; Schoepfer, E. Classifier directed data transformations in sample supervised segment generation, Paper presented at Geographic Object-based Image Analysis (GEOBIA 2014), Thessaloniki, Greece, 21-24 May.

Fourie, C.; Schoepfer, E. Connectivity thresholds and data transformations for sample supervised segment generation, Paper presented at Geoscience and Remote Sensing Symposium (IGARSS 2013), IEEE International, Melbourne, 21-26 July.

Fourie, C, Van Niekerk, A \& Mucina, L 2012, ‘Semi-automated segment generation for geographic novelty detection using edge and area metrics', South African Journal of Geomatics, Vol 1(2), pp. 133-148.

Fourie, C.; Schoepfer, E. Combining the heuristic and spectral domains in semi-automated segment generation, Paper presented at Geographic Object-based Image Analysis (GEOBIA 2012), Brazil, 7-9 May 2012.

Fourie, C.; Van Niekerk, A.; Mucina, L. Optimising a one-class svm for geographic object-based novelty detection, Paper presented at AfricaGeo, Cape Town, South Africa, 31 May - 2 June 2011.

Fourie, C. A one class object based system for sparse geographic feature identification. Stellenbosch University, Stellenbosch, 2011.

Jena,

Place, Date

Christoffel Ettienne Fourie 


\section{STATEMENT OF AUTHORSHIP}

Selbstständigkeitserklärung:

Ich erkläre, dass ich die vorliegende Arbeit selbstständig und unter Verwendung der angegebenen Hilfsmittel, persönlichen Mitteilungen und Quellen angefertigt habe.

Jena,

Ort, Datum

Christoffel Ettienne Fourie 